Nino Raddao

Potenzialorientierte Kundensegmentierung zur Optimierung des Leistungsportfolios in der Firmenkundenbank 
Nino Raddao

\section{Potenzialorientierte Kundensegmentierung zur Optimierung des Leistungsportfolios in der Firmenkundenbank}

Aufgrund der hohen Substituierbarkeit klassischer Bankprodukte kommt dem Management der Kundenbeziehung als Differenzierungsstrategie im Firmenkundengeschäft eine entscheidende Bedeutung zu. Eine wesentliche Herausforderung stellt dabei die Ausgestaltung des Leistungsangebotes der Bank dar. Einerseits ist die Kunde-Bank-Beziehung so zu gestalten, dass ein erlebbarer Mehrwert für den Firmenkunden entsteht. Andererseits muss dabei bankseitig auch eine angemessene Rentabilität der Geschäftsbeziehung und ein effizienter Einsatz der Betreuungsressourcen gewährleistet werden. In diesem Beitrag - der auf der mit dem DZ BANK Karriere-Preis ausgezeichneten Diplomarbeit des Autors basiert - wird ein multidimensionales Segmentierungsmodell entwickelt, das als Ausgangspunkt eines ganzheitlichen Vertriebsmanagements verstanden werden kann. Der Autor verknüpft den entwickelten Ansatz mit einer empirischen Analyse zur Anforderungsstruktur von Firmenkunden und leitet daraus Implikationen zur segmentspezifischen Optimierung des Leistungsportfolios von Banken ab.

Nino Raddao absolvierte parallel zu seiner Ausbildung zum Bankkaufmann mit anschließendem Traineeprogramm ein berufbegleitendes Studium der Betriebswirtschaftslehre an der FHDW - Fachhochschule der Wirtschaft. In der Bank leitete er ein Großprojekt im Bereich Vertriebsmanagement und war anschließend als Customer Relationship Manager tätig. Anfang 2008 wechselte der Autor zu einer Unternehmensberatung mit Schwerpunkt Bankenberatung. Zudem hat er kürzlich ein Promotionsstudium an der Westflälischen Wilhelms-Universität Münster aufgenommen. 
Potenzialorientierte Kundensegmentierung zur Optimierung des Leistungsportfolios in der Firmenkundenbank 


\section{Beiträge zum Controlling}

Herausgegeben von Wolfgang Berens

Band 13

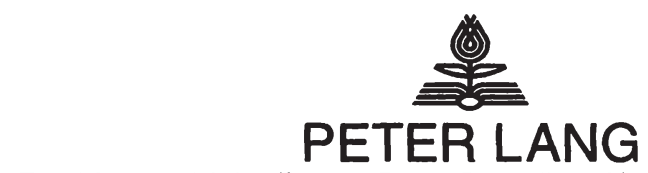

Frankfurt am Main - Berlin - Bern - Bruxelles - New York - Oxford · Wien

Nino Raddao - 978-3-631-75326-2

Downloaded from PubFactory at 01/11/2019 06:11:43AM

via free access 
Nino Raddao

\section{Potenzialorientierte \\ Kundensegmentierung \\ zur Optimierung \\ des Leistungsportfolios \\ in der Firmenkundenbank}

Konzeption und Implementierung

einer efficient customization am Beispiel

von Genossenschaftsbanken

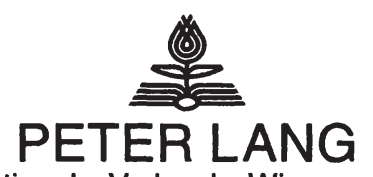

Internationaler Verlag derWissenschaften 
Bibliografische Information der Deutschen Nationalbibliothek Die Deutsche Nationalbibliothek verzeichnet diese Publikation in der Deutschen Nationalbibliografie; detaillierte bibliografische Daten sind im Internet über <http://www.d-nb.de> abrufbar.

Open Access: The online version of this publication is published on www.peterlang.com and www.econstor.eu under the international Creative Commons License CC-BY 4.0. Learn more on how you can use and share this work: http://creativecommons.org/licenses/ by/4.0.

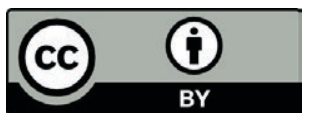

This book is available Open Access thanks to the kind support of ZBW - Leibniz-Informationszentrum Wirtschaft.

Gedruckt auf alterungsbeständigem, säurefreiem Papier.

\author{
ISSN 1618-825X \\ ISBN 978-3-631-58354-8 \\ ISBN 978-3-631-75326-2 (eBook) \\ (c) Peter Lang GmbH \\ Internationaler Verlag der Wissenschaften \\ Frankfurt am Main 2009 \\ Alle Rechte vorbehalten.
}

Das Werk einschließlich aller seiner Teile ist urheberrechtlich geschützt. Jede Verwertung außerhalb der engen Grenzen des

Urheberrechtsgesetzes ist ohne Zustimmung des Verlages unzulässig und strafbar. Das gilt insbesondere für

Vervielfältigungen, Übersetzungen, Mikroverfilmungen und die Einspeicherung und Verarbeitung in elektronischen Systemen.

Printed in Germany 123457

www.peterlang.de 
Für Miranda

Nino Raddao - 978-3-631-75326-2

Downloaded from PubFactory at 01/11/2019 06:11:43AM

via free access 
Nino Raddao - 978-3-631-75326-2

Downloaded from PubFactory at 01/11/2019 06:11:43AM

via free access 


\section{Geleitwort}

Deutsche Kreditinstitute - insbesondere Regionalbanken - bewegen sich derzeit in einem schwierigen Marktumfeld. Während einerseits die gestiegene Preissensitivität der Bankkunden im Kontext einer hohen Markttransparenz zu einem erhöhten Margendruck führt, wird die Ertragssituation zusätzlich durch makroökonomische Rahmenparameter (flache Zinsstrukturkurve, etc.) negativ beeinflusst.

Besonders im beratungsintensiven Firmenkundengeschäft ist daher eine Differenzierungsstrategie erforderlich, die eine Wettbewerbsabgrenzung ermöglicht ohne Rentabilitäts- und Effizienzaspekte außer Acht zu lassen. Ausgehend von dieser Herausforderung stellt das vorliegende Werk einen innovativen Segmentierungsansatz vor, der weit über die bisher in Literatur und Praxis verwendeten Klassifizierungsverfahren hinausgeht. Die Segmentierung wird von einem reinen Clustering der strategischen Geschäftseinheit Firmenkunden zu einem zentralen Instrument des Vertriebsmanagements weiterentwickelt. Herr RADDAO prägt in diesem Zusammenhang den Begriff der „efficient customization“, der eine konsequente Ausrichtung der Betreuungsindividualität und -intensität an den Kundenanforderungen, sowie der Rentabilität und des Potenzials der Geschäftsbeziehung meint. Erreicht wird dies über einen segmentspezifischen Zuschnitt des Leistungsportfolios.

Erkenntnisunterstützend hat Herr RADDAO eine umfangreiche empirische Studie zur Anforderungsstruktur im Firmenkundengeschäft durchgeführt. Dabei ist es ihm unter Verwendung des - in der deutschsprachigen Literatur noch weitgehend unbekannten Importance Grid auf eindrucksvolle Weise gelungen, die Kundenanforderungen an das Leistungsportfolio und die Geschäftsbeziehung nach Basis-, Leistungs- und Begeisterungsanforderungen zu klassifizieren. Die Arbeit wird durch Verknüpfung der Segmentierungskonzeption mit den Erkenntnissen der empirischen Untersuchung unter Derivation segmentspezifischer Normstrategien sinnvoll abgerundet.

Besonders hervorzuheben sind der hohe praktische Anwendungsbezug, die breite Verwendbarkeit, sowie die sorgfältige wissenschaftliche und empirische Fundierung des vorliegenden Werkes, welche nicht zuletzt durch die Auszeichnung der zugrundeliegenden Diplomarbeit mit dem $D Z B A N K$ Karriere-Preis bestätigt wird. Ich wünsche der Monographie daher eine weite Verbreitung in Wissenschaft und Praxis.

Münster, im Juli 2008

Prof. Dr. Wolfgang Berens 


\section{Vorwort}

Das vorliegende Werk ist auf Grundlage meiner Diplomarbeit entstanden, welche im Februar 2007 unter dem Titel „Potenzialorientierte Kundensegmentierung zur Optimierung des Leistungsportfolios einer ganzheitlichen Firmenkundenbetreuung genossenschaftlicher Kreditinstitute" als Abschlussarbeit meines berufsbegleitenden Studiums der Betriebswirtschaftslehre an der FHDW - Fachhochschule der Wirtschaft eingereicht wurde. In diesem Zusammenhang gilt mein ganz besonderer Dank meinem akademischen Lehrer Herrn Prof. Dr. THOMAS JENSEN für die Betreuung und die Übernahme des Erstgutachtens. Sein Engagement im Hinblick auf die Begleitung des Erstellungsprozesses ging weit über ein zu erwartendes $\mathrm{Ma} ß$ hinaus und hat wesentlich zum Gelingen der Diplomarbeit beigetragen. Für die Übernahme des Zweitgutachtens danke ich Herrn Prof. Dr. HeINER LANGEMEYER.

Eine besondere Anerkennung möchte ich Herrn THOMAS KERSTING B.Sc., Herrn Dipl.-Bankbetrw. (ADG) RALF ReCKMEYeR, Herrn Dr. Klaus SEgBers und Herrn Dr. ANDREAS SIEMES zu Teil werden lassen.

Herrn THOMAS KERSTING danke ich für sein - für einen Informatiker - ausgeprägtes Interesse an wirtschaftlichen Zusammenhängen, die vielen Gespräche auf hohem Abstraktionsniveau, seine Funktion als Motivator und Freund, sowie insbesondere für die prototypische softwaretechnische Umsetzung des Segmentierungskonzeptes. Bei Herrn RALF RECKMEYER möchte ich mich für seinen Innovationsdrang, seine Begeisterung für die Thematik, seine Offenheit und den fundierten Einsatz seines Expertenwissens bedanken. Herrn Dr. KLAUS SEGBERS danke ich für die Zurverfügungstellung seiner Dissertationsschrift, die engagierte Unterstützung und den konstruktiven Diskurs zur Empirie und den verhaltenswissenschaftlichen Aspekten der Kunde-BankBeziehung. Herrn Dr. ANDREAS SIEMES gebührt spezieller Dank, da er aufstrebenden Akademikern in bemerkenswerter Art und Weise die Möglichkeit und den Freiraum gibt, in einem hochmotivierten, exzellent besetzten Team konzeptionelle Ideen zur Marktreife weiterzuentwickeln. Es ehrt mich sehr, gemeinsam mit ihm, Herrn Dr. SEGBERS und weiteren Kollegen zukunftsorientiert an der Weiterentwicklung des Firmenkundengeschäfts arbeiten zu dürfen.

Für die konstruktiven Diskussionen zur fachlichen und praktischen Fundierung und die allgemeine Unterstützung danke ich auch Herrn Dipl.-Bankbetrw. (ADG) / Dipl.-Kfm. (FH) FRANK BÖGER, Herrn Dipl.-Bankbetrw. (ADG) / Dipl.-Kfm. MichaEL DEITERT und Herrn Dipl.-Bankbetrw. (ADG) HANS MESTEKEMPER.

Desweiteren möchte ich Herrn Prof. Dr. WOLFGANG BERENS herzlich für die Aufnahme des vorliegenden Werkes in seine Schriftenreihe, sowie sein Vertrauen und die Begleitung meines Dissertationsvorhabens danken. Es freut mich sehr, meine akademische Laufbahn am Lehrstuhl für Betriebswirtschaftslehre insb. Controlling der Westfälischen Wilhelms-Universität Münster fortsetzen zu dürfen. 
Ein ganz persönliches Dankeschön möchte ich an die Menschen richten, die mir den Rückhalt in allen privaten und beruflichen Belangen geben. Ohne ihren Einsatz, ihre Zuneigung und ihr Verständnis wären das Gelingen der Diplomarbeit sowie die Bewältigung der sonstigen Herausforderungen in meinem Leben unmöglich gewesen.

Zunächst sind hier meine Eltern ORTRUD und UMBERTO RADDAO, sowie mein Bruder RıCO zu nennen, die immer für mich da sind und deren selbstloser Hilfe ich mir - vor allem in schwierigen Zeiten - sicher sein kann. Für die besondere Fürsorge in Kinderund Jugendtagen danke ich meiner leider schwer erkrankten Großmutter ANNA RÖNNAU und meinem Großvater HELMUT RÖNNAU. Für die Unterstützung aus der Ferne gilt mein Dank meinen bedauerlicherweise bereits verstorbenen Großeltern GIACOMA und GAETANO RADDAO. Zum Abschluss möchte ich mich ganz besonders herzlich bei meiner Freundin MIRANDA PELlicCIOTTA bedanken. Unsere Beziehung war durch den fordernden Bankalltag in Kombination mit dem mehrjährigen Abendund Wochenendstudium an der $F H D W$ einer Vielzahl von Entbehrungen ausgesetzt. MIRANDAS bedingungsloses Verständnis, ihre permanente Unterstützung und ihre Liebe ebneten in dieser Zeit jeden noch so steinigen Weg. Ich hoffe, ihr dies in der Zukunft stets zurückgeben zu können. Die vorliegende Monographie widme ich daher ihr und meiner Familie. 
Nino Raddao - 978-3-631-75326-2

Downloaded from PubFactory at 01/11/2019 06:11:43AM

via free access 


\section{Inhaltsverzeichnis}

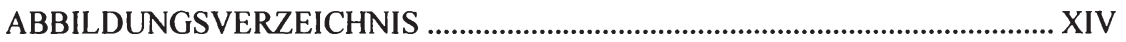

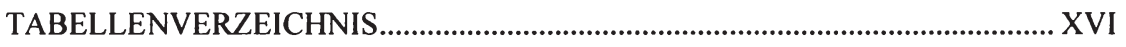

ABKÜRZUNGSVERZEICHNIS ..................................................................XVII

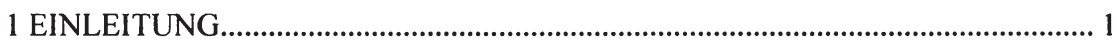

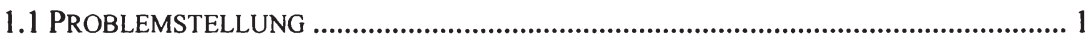

1.2 ZIELSETZUNG DER ARBEIT ................................................................................. 1

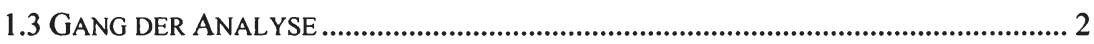

2 VORSTELLUNG DER GENOSSENSCHAFTLICHEN BANKENGRUPPE ........... 4

2.1 Die genossensChaftliche BankengrupPe IM PRofiL....................................... 4

2.2 STRATEGISCHE AUSRICHTUNG IM FIRMENKUNDENGESCHÄFT ................................ 5

3 FIRMENKUNDENGESCHÄFT IM WANDEL ………............................................. 7

3.1 WETTBEWERBSPOSITION GENOSSENSCHAFTLICHER KREDITINSTITUTE ................. 7

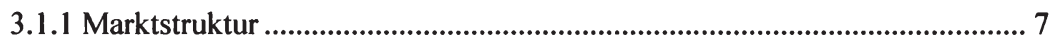

3.1.2 Wettbewerbssituation im Firmenkundengeschäft ..................................... 8

3.1.3 Kundenstruktur im Firmenkundengeschäft ................................................. 12

3.1.4 SWOT-Analyse für das genossenschaftliche Firmenkundengeschäft........ 14

3.2 NOTWENDIGKEIT ZUR DIFFERENZIERUNG IM WETTBEWERB................................ 15

3.2.1 Homogenität von Bankprodukten.......................................................... 15

3.2.2 Abwanderungsanalyse im Firmenkundengeschäft ...................................... 17

\section{KUNDENBINDUNGSMANAGEMENT ALS}

DIFFERENZIERUNGSMERKMAL _................................................................ 18

4.1 STRATEGISCHE BEDEUTUNG DER KUNDENBINDUNG ......................................... 18

4.2 DETERMINANTEN EINER INTENSIVEN KUNDENBINDUNG ..................................... 19

4.2.1 Wirkmodell der Kundenbindung im Firmenkundengeschäft ....................... 19

4.2.2 Kundenanforderungen im Wirkzusammenhang ....................................... 22 
4.2.3 Vertrauen als zentrales Element des Wirkmodells ...................................... 23

4.3 KUndENBINDUNGSMANAgEMENT AUS STRATEgISCHER PERSPEKTIVE................ 25

4.3.1 Begriffliche Abgrenzung des Kundenbindungsmanagements ................... 25

4.3.2 Parameter eines integrierten Kundenbindungsmanagements ...................... 26

5 OPERATIONALISIERUNG DER DIFFERENZIERUNGSSTRATEGIE

DURCH POTENZIAL- UND BEDARFSORIENTIERUNG....................................29

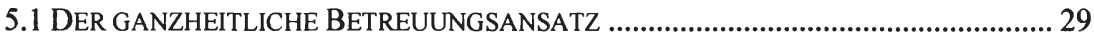

5.1.1 Systematik und Intention einer ganzheitlichen Firmenkundenbetreuung .. 29

5.1.2 Grenzen der Ganzheitlichkeit ................................................................. 33

5.2 DiE PROBLEMATIK EINER EFFIZIENTEN RESSOURCENALLOKATION....................... 34

5.2.1 Zeitprofil eines Firmenkundenbetreuers.................................................... 34

5.2.2 Produktivitätssteigerung durch Erhöhung der Nettomarktzeit .................... 36

6 METHODISCHE INSTRUMENTARIEN ZUR SEGMENTIERUNG EINES

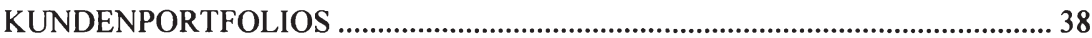

6.1 GRUNDLAGEN DER KUNDENSEGMENTIERUNG ..................................................... 38

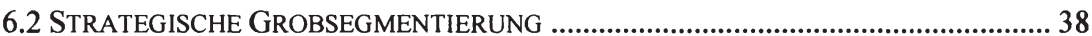

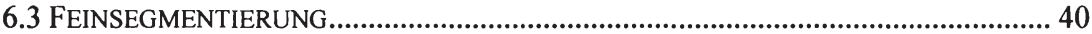

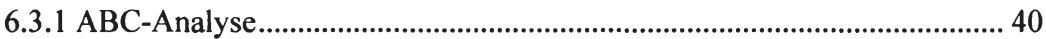

6.3.2 Portfolio-Analyse............................................................................................... 41

7 POTENZIALORIENTIERTE KUNDENSEGMENTIERUNG .................................46

7.1 ANFORDERUNGSANALYSE UND ZIELDEFINITION ................................................. 46

7.2 ENTWICKLUNG EINES PORTFOLIO-MODELLS IM FIRMENKUNDENGESCHÄFT ...... 47

7.2.1 Konzept und Dimensionierung ........................................................................ 47

7.2.2 Kriterien zur Erhebung des aktuellen Status ................................................ 48

7.2.3 Kriterien zur Erhebung des Potenzials ........................................................ 51

7.2.4 Kriterienscoring ............................................................................................... 51 
7.2.5 Portfolio-Positionierung und Segmentierungsstufen...................................... 54

7.3 RESSOURCENBINDUNG UND RISIKOSITUATION ................................................55

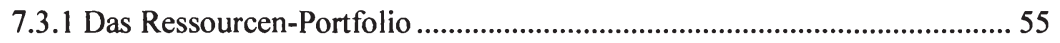

7.3.2 Das Risiko-Portfolio .................................................................................. 57

7.4 KONSEQUENZEN FÜR DAS VERTRIEBSMANAGEMENT ........................................... 57

7.4.1 Wechselwirkungen der Kundensegmentierung im Vertriebsprozess......... 59

7.4.2 Validität der Segmentierungsergebnisse ........................................................ 60

\section{ANFORDERUNGSSTRUKTUREN IM FIRMENKUNDENGESCHÄFT}

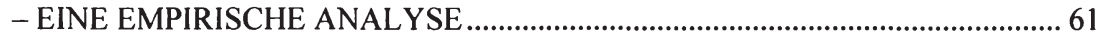

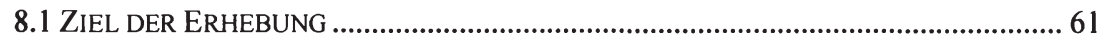

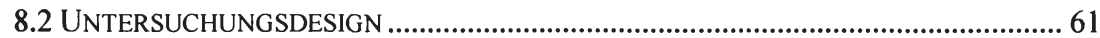

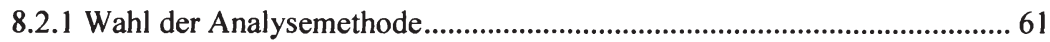

8.2.2 Fragebogendesign und Struktur der Analyse................................................ 65

8.2.3 Angaben zur Datenerhebung ...................................................................... 67

8.3 ANWENDUNG DER IMPORTANCE GRID-METHODE ..............................................68

8.3.1 Festlegung der Untersuchungshypothesen ..................................................6 68

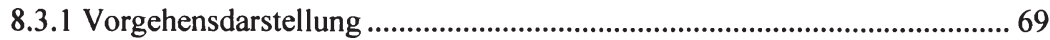

8.4 ERGEBNISINTERPRETATION UNTER EINBEZUG DER NEBENUNTERSUCHUNGEN... 73

8.4.1 Interpretation der Dienstleistungsfaktoren ................................................ 73

8.4.2 Interpretation der Beziehungsfaktoren .......................................................... 74

9 OPTIMIERUNG DES LEISTUNGSPORTFOLIOS ............................................. 76

9.1 SEgMENTSPEZIFISCHE FESTLEGUNG DES LEISTUNGSANGEBOTES........................ 76

9.2 GEZIELTE RESSOURCENSTEUERUNG JE SEGMENT ................................................ 77

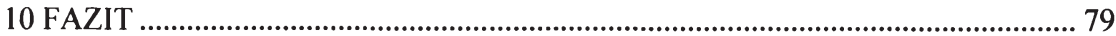

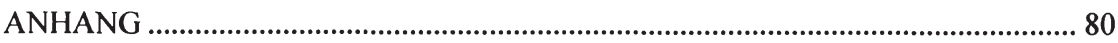

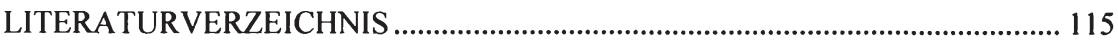




\section{Abbildungsverzeichnis}

Abbildung 1: Gang der Analyse ................................................................................. 2

Abbildung 2: Strategische Stoßrichtung des VR-Finanzplan Mittelstand ......................... 6

Abbildung 3: Kundenreichweite und Marktanteile im deutschen

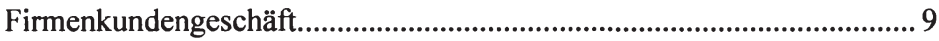

Abbildung 4: Entwicklung der Zinsmargen.................................................................... 11

Abbildung 5: Triebkräfte der Internationalisierung des Mittelstandes.......................... 13

Abbildung 6: SWOT-Analyse für das genossenschaftliche Firmenkundengeschäft.... 14

Abbildung 7: Positionierungsmodell deutscher Banken................................................ 16

Abbildung 8: Wirkmodell einer Bankbeziehung mittelständischer Unternehmen....... 20

Abbildung 9: Schichtenmodell der Kundenbindung ...................................................... 21

Abbildung 10: Zufriedenheitswirkung von Kundenanforderungen ............................... 22

Abbildung 11: Parameter eines integrierten Kundenbindungsmanagements ................ 28

Abbildung 12: Bedarfsfelder im Firmenkundengeschäft ................................................ 30

Abbildung 13: Das Strategiegespräch im Ablaufmodell ................................................ 31

Abbildung 14: Aufgaben des Firmenkundenbetreuers nach Vertriebsorientierung ..... 35

Abbildung 15: Ressourcenverwendung der Firmenkundenkundenbetreuer.................. 36

Abbildung 16: Nettomarktzeiten der Firmenkundenbetreuer nach Bankrentabilität ... 36

Abbildung 17: Firmenkundenportfolio nach SCHMOLL .................................................. 44

Abbildung 18: Kriterium Deckungsbeitrag - Merkmalsausprägung................................ 52

Abbildung 19: Betriebliche Leistungsanalyse ............................................................. 53

Abbildung 20: Private Leistungsanalyse ....................................................................... 53

Abbildung 21: Qualitative Kriterien: Merkmalsausprägungen ..................................... 54

Abbildung 22: Engagement-Portfolio........................................................................... 55

Abbildung 23: Ressourcen-Portfolio .............................................................................. 56

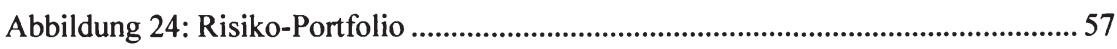

Abbildung 25: Regelkreis einer zweistufigen Kundensegmentierung .......................... 59

Abbildung 26: Das Importance Grid ............................................................................. 64

Abbildung 27: Hypothesenschema zur Analyse von Begeisterungsanforderungen..... 68

Abbildung 28: Importance Grid: Dienstleistungsfaktoren 
Abbildung 29: Importance Grid: Beziehungsfaktoren ........................................... 73

Abbildung 30: Firmenkunden-Portfolio: Leistungsstrategien ........................................ 76

Abbildung 31: Firmenkundenportfolio: Ressourcensteuerung...................................... 77

Abbildung 32: Firmenkunden einer Genossenschaftsbank nach Sektoren..................... 81

Abbildung 33: Wettbewerbsstrategien nach PORTER .................................................... 82

Abbildung 34: Anforderungsdimensionen in Hausbankbeziehungen ............................ 83

Abbildung 35: Ratinganalyse in MinD.banker ................................................................. 84

Abbildung 36: Analyseschritte und inhaltliche Vorgehensweise ................................... 85

Abbildung 37: Cluster-Bildung ........................................................................................ 85

Abbildung 38: Bedarfsprofile je Kundensegment ......................................................... 86

Abbildung 39: Übersicht - Typologisierung der Segmentierungskriterien................... 87

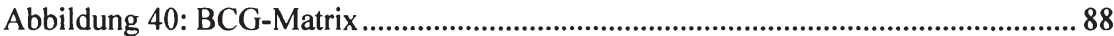

Abbildung 41: Das Wettbewerbsvorteils-Marktattraktivitäts-Portfolio .......................... 89

Abbildung 42: Normstrategien des McKinsey-Portfolios ............................................... 90

Abbildung 43: Kundenportfolio-Matrix nach SCHRÖDER ....................................... 92

Abbildung 44: Betriebliche Umfeldfaktoren: Merkmalausprägungen ............................ 94

Abbildung 45: Private Umfeldfaktoren: Merkmalsausprägungen.................................. 94

Abbildung 46: Bewertungsprofil Statusscoring........................................................... 95

Abbildung 47: Bewertungsprofil Potenzialscoring......................................................... 96

Abbildung 48: Bewertungsprofil Risikoscoring ............................................................. 97

Abbildung 49: Bewertungsprofil Ressourcenscoring ...................................................... 97

Abbildung 50: Kreisdiagramm: Sektorzugehörigkeit.................................................. 108

Abbildung 51: Hypothesenschema zur Überprüfung von Basisanforderungen .......... 110

Abbildung 52: Hypothesenschema zur Überprüfung von (wichtigen)

Leistungsanforderungen

Abbildung 53: Hypothesenschema zur Überprüfung von (unwichtigen)

Leistungsanforderungen.

Abbildung 54: Hypothesenschema zur Überprüfung von

Begeisterungsanforderungen 


\section{Tabellenverzeichnis}

Tabelle 1: Segmentzuordnung in der Firmenkundenbetreuung gem. BVR ................... 5

Tabelle 2: Anwesenheitszeit eines Firmenkundenbetreuers............................................. 35

Tabelle 3: Segmentierungskriterien nach SCHMOLL........................................................ 43

Tabelle 4: Segmentierungskriterien zur Statuserhebung .................................................. 49

Tabelle 5: Explizite und implizite Wichtigkeit von Kundenanforderungen.................... 63

Tabelle 6: Struktur der empirischen Analyse ..................................................................66

Tabelle 7: Umsatzstruktur der Stichprobe ....................................................................... 67

Tabelle 8: Faktorenzuordnung Dienstleistungen (geordnet nach Faktorladungen)...... 71

Tabelle 9: Faktorenzuordnung Beziehungsattribute (geordnet nach Faktorladungen) 72

Tabelle 10: Quantitative Mittelstandsdefinition des IfM Bonn ....................................... 81

Tabelle 11: Die Positionierungsfelder der BCG-Matrix............................................... 88

Tabelle 12: Multifaktoren-Scoring nach McKinsey ........................................................ 91

Tabelle 13: Segmentierungskriterien zur Potenzialerhebung .......................................... 93

Tabelle 14: Sektorzugehörigkeit der Stichprobe .......................................................... 108

Tabelle 15: Eigenkapitalstruktur der Stichprobe ......................................................... 108

Tabelle 16: Stichprobenstruktur: Anzahl aktiver Kontoverbindungen.......................... 108

Tabelle 17: Strichprobenstruktur: Banken mit Hausbankstatus ................................... 109

Tabelle 18: Leistungsangebot und -inanspruchnahme bei verschiedenen

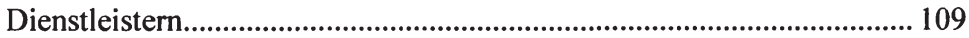

Tabelle 19: Korrelationsanalyse: Betriebswirtschaftliches Serviceangebot................ 114 

Abkürzungsverzeichnis
AG
Aktiengesellschaft
BMS
BMS Consulting $\mathrm{GmbH}$
BVR
Bundesverband der deutschen Volksbanken und Raiffeisenbanken
CIR
Cost-Income-Ratio, Aufwandsrentabilität
DIN
Deutsches Institut für Normung
DSGV
Deutscher Sparkassen- und Giroverband
EN
Europäische Norm
GenG
Genossenschaftsgesetz
IfM Bonn
Institut für Mittelstandsforschung Bonn
ISO
International Standardization Organization
KWG
Kreditwesengesetz
ROE
Return on Equity, Eigenkapitalrentabilität
SB
Selbstbedienung
SGE
Strategische Geschäftseinheit
USP
Unique Selling Proposition 
Nino Raddao - 978-3-631-75326-2

Downloaded from PubFactory at 01/11/2019 06:11:43AM

via free access 


\section{Einleitung}

\subsection{Problemstellung}

Ansätze zur Kundensegmentierung erfreuen sich im Bankwesen schon seit geraumer Zeit einer regen Anwendung. 'Allerdings handelt es sich bei den gängigen Verfahren, um eine eher grobe Klassifizierung des Kundenportfolios einer Geschäftseinheit, die einen eher strategischen Charakter aufweist. Zudem existieren nur wenige, speziell für das Firmenkundengeschäft entwickelte Modelle.

Für eine differenzierte Marktbearbeitung reicht eine solche Grobeinteilung jedoch nicht aus. Vielmehr müssen die Vertriebsressourcen so gesteuert werden, dass insgesamt eine angemessene Rentabilität des Firmenkundengeschäftes gewährleistet wird.

Bei einem solchen rational-ökonomischen Ansatz darf jedoch nicht vergessen werden, dass eine partnerschaftliche Kunde-Bank-Beziehung die Grundlage des Vertriebserfolges darstellt. In diesem Zusammenhang hat der Begriff der Kundenbindung in den letzten Jahren an Bedeutung gewonnen. ${ }^{2}$ Diese Entwicklung ist auch auf den sich zunehmend verschärfenden Wettbewerb im Firmenkundengeschäft zurückzuführen.

Empirische Studien weisen sowohl der Kundenbindung als auch einer detaillierten Kundensegmentierung einen entscheidenden Einfluss auf den Vertriebserfolg nach. ${ }^{3}$ Problematisch erscheint allerdings die Verknüpfung dieser beiden - auf den ersten Blick konfliktär - erscheinenden Erfolgsfaktoren.

Um einen nachhaltigen Wettbewerbsvorteil zu erlangen ist zudem eine genaue Kenntnis der Kundenanforderungen erforderlich. Die Analyse der Anforderungsstruktur stellt dabei die Grundlage einer bedarfsorientierten Ausgestaltung des Leistungsportfolios dar.

\subsection{Zielsetzung der Arbeit}

In der vorliegenden Arbeit soll am Beispiel der Genossenschaftsbanken, die Entwicklung eines potenzialorientierten Segmentierungsansatzes vorgestellt werden, der als Ausgangspunkt eines integrierten Vertriebsmanagements verstanden werden kann, indem eine optimale Verzahnung aller relevanten Instrumente erreicht wird. Die Fokussierung auf eine Bankengruppe erfolgt dabei, da es sinnvoll und notwendig erscheint bei einer detaillierten Kundenklassifizierung bankengruppenspezifische Besonderheiten zu berücksichtigen. Hier sind insbesondere Rahmenparameter, wie die grundsätzliche strategische Ausrichtung, die Vertriebs- und Betreuungskonzeption

Vgl. Bufka, Jürgen / Eichelmann, Thomas (2002), S. 125

Vgl. u.a. Bruhn, Manfred / Homburg, Christian (2005), S. 5 f.

Vgl. Käser, Burkhard et al. (2004), S. 12 f. 
sowie der Beratungsansatz zu nennen. Das zu entwickelnde Verfahren soll allerdings hinreichend flexibel und adjustierbar sein, damit auch institutsindividuelle Spezifika einfließen können.

Parallel erfolgt eine Analyse der Anforderungsstruktur im Firmenkundengeschäft, um daraus überblicksartig erste Implikationen zur Optimierung des Leistungsportfolios abzuleiten. Es soll dabei auch erklärt werden, inwiefern die Segmentierungsergebnisse hier sinnvoll einfließen können.

\subsection{Gang der Analyse}

Um die sich ergebenden Schnittstellen adäquat zu würdigen, wurden die zu untersuchenden Sachverhalte bewusst einer umfassenden, den Gesamtkontext berücksichtigenden Analyse unterzogen werden. Abbildung 1 veranschaulicht die Vorgehensweise:

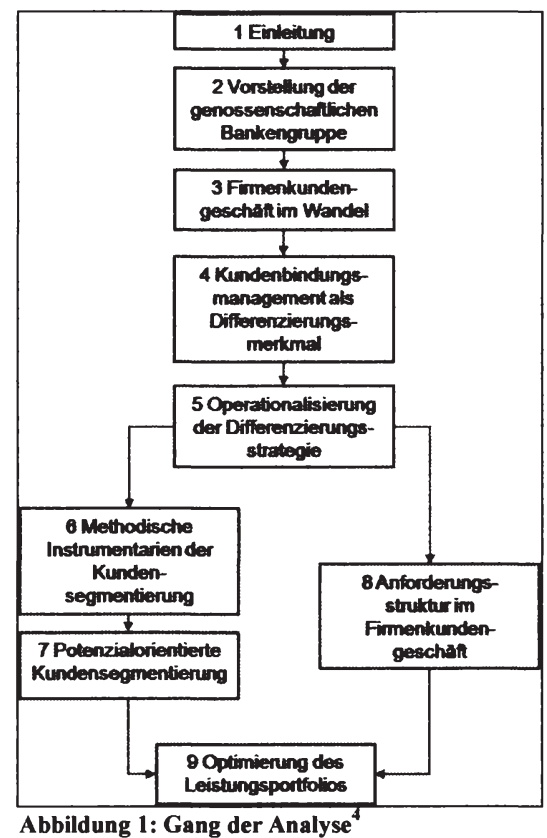

$4 \quad$ Eigene Darstellung 
Nachdem in diesem ersten Kapitel die grundsätzliche Notwendigkeit einer differenzierten Kundensegmentierung aufgezeigt und auf die Wichtigkeit ihrer umfassenden Integration hingewiesen wurde, soll in Kapitel 2 eine kurze Vorstellung der genossenschaftlichen Bankengruppe erfolgen, so dass die spezifischen Charakteristika im weiteren Verlauf konzeptionell berücksichtigt werden können.

Im dritten Kapitel werden nach einer überblicksartigen Darstellung der Marktstruktur, die Wettbewerbsbedingungen im Firmenkundegeschäft und die Notwendigkeit zur Differenzierung erörtert, um im vierten Kapitel das Kundenbindungsmanagement als geeignete Differenzierungsstrategie herauszuarbeiten. Wie sich zeigen wird, kann diese in Kapitel 5 durch den ganzheitlichen Betreuungsansatz operationalisiert werden. Dabei ist neben der Bedarfssituation auch die Ertrags- und Ressourcensituation der Firmenkunden zu berücksichtigen.

Um dies zu erreichen wird im siebten Kapitel - nach der Diskussion einiger methodischer Ansätze zur Segmentierung eines Kundenportfolios in Kapitel 6 - ein Segmentierungsverfahren entwickelt werden, welches einerseits eine Differenzierung ermöglicht und andererseits eine angemessene Rentabilität und einen effizienten Ressourceneinsatz gewährleistet.

Kapitel 8 befasst sich mit der Auswertung der empirischen Analyse zur Anforderungsstruktur im Firmenkundengeschäft. Diese dient zur Intensivierung der Differenzierungswirkung im Zuge eines gezielten, an den Kundenanforderungen ausgerichteten Einsatzes des Leistungsportfolios.

Im neunten Kapitel der vorliegenden Arbeit sollen die gewonnen Erkenntnisse dazu genutzt werden, überblicksartig erste Implikationen zur Optimierung des Leistungsportfolios abzuleiten. Dies bezieht sich nicht nur auf das angebotene Produktportfolio, sondern vielmehr auf die gesamte Betreuungsleistung. Die Arbeit endet mit einem abschließenden Gesamtfazit. 


\section{Vorstellung der genossenschaftlichen Bankengruppe}

\subsection{Die genossenschaftliche Bankengruppe im Profil}

Der genossenschaftliche Bankensektor ist in einem Finanzverbund organisiert. Dieser besteht aus einem Vertriebsnetz von 1.390 Primärbanken ${ }^{5}$, zwei Spitzeninstituten ${ }^{6}$, diversen - auf bestimmte Finanzdienstleistungen spezialisierten - Verbundunternehmen ${ }^{7}$, sowie Technik- und IT-Dienstleistern'.

Die Volks- und Raiffeisenbanken sind Allfinanzunternehmen, die über den genossenschaftlichen FinanzVerbund mit einem universellen Leistungsangebot am Markt präsent sind. Zwei wesentliche Charakteristika des Genossenschaftssektors sind in der dezentralen Organisation und der Subsidiarität zu sehen'. Durch die autonom agierenden und rechtlich selbständigen Primärbanken wird eine starke regionale Präsenz und Kundennähe erreicht.

Insgesamt werden rund 30 Millionen Privat- und Firmenkunden betreut. Davon sind 15,7 Millionen als Mitglieder zugleich Miteigentümer, Kapitalgeber und Gewinnbeteiligte ihrer Primärbank. Neben ökonomischen Zielen ist die Förderung der Mitglieder das Kernziel der Genossenschaftsbanken. ${ }^{10}$

Die Interessen der genossenschaftlichen Bankengruppe werden auf nationaler Ebene vom Bundesverband der Deutschen Volksbanken und Raiffeisenbanken e.V. - kurz $B V R$ - vertreten. Zusätzlich zu seiner repräsentativen Funktion besteht die Hauptaufgabe in der Koordination und Entwicklung einer gemeinschaftlichen Strategie der Volks- und Raiffeisenbanken." Neben dem $B V R$ als Spitzenverband existieren noch sieben Regionalverbände. ${ }^{12}$

5 Die 1390 Primärbanken betreiben national ca. 14.000 Bankstellen, vgl. BVR (2006a).

6 Bei den Kopfinstituten des Genossenschaftssektors handelt es sich um DZ BANK $A G$ in Frankfurt am Main und die WGZ-Bank AG Westdeutsche Genossenschafts-Zentralbank in Düsseldorf, vgl. BVR (2006b).

$7 \mathrm{Zu}$ den Verbundunternehmen, die banknahe Finanzdienstleistungen anbieten, gehören z.B. die $R+V$ Versicherung $A G$ in Wiesbaden und die VR-LEASING $A G$ in Eschborn, vgl. BVR (2006c).

8 Als wichtigste Vertreter sind hier die genossenschaftlichen Rechenzentren, die GAD eG Gesellschaft für automatische Datenverarbeitung in Münster und die FIDUCIA IT AG in Karlsruhe, vgl. BVR (2006c).

9 Im genossenschaftlichen FinanzVerbund übernimmt nach dem Subsidiaritätsprinzip die organisatorisch höher angesiedelte Ebene (Zentralbank, Verbundpartner) nur Aufgaben, welche die niedrige (Primärbank) nicht oder nicht ausreichend erfüllen kann (z.B. aufgrund regulatorischer Restriktionen), vgl. Strauß, Marc-R. (2006), S. 25 f..

Vgl. BVR (2006b) 


\subsection{Strategische Ausrichtung im Firmenkundengeschäft}

Unter dem Titel „Bündelung der Kräfte“ hat der BVR im Jahr 2001 eine Strategie für eine nachhaltig erfolgreiche Marktbearbeitung und Wettbewerbsdifferenzierung verabschiedet. ${ }^{13}$ Aus dem in sieben Teilprojekte untergliederten Großprojekt, ist das Firmenkundengeschäft insbesondere von den folgenden Anforderungen tangiert:

- Segmentierung der Firmenkunden

- Implementierung einer ganzheitlichen Vertriebs- und Betreuungskonzeption

- Straffung, Standardisierung und Weiterentwicklung der Produktpalette

- Integration des Firmenkundengeschäftes in eine Vertriebswegestrategie ${ }^{14}$

Aufgrund der besonderen Bedeutung der beiden erstgenannten Handlungsfelder soll auf diese gesondert eingegangen werden.

Der BVR hat bereits mit dem Teilprojekt „Segmentierung der Kunden der Volksbanken und Raiffeisenbanken " die Wichtigkeit einer systematischen Kundensegmentierung erkannt. Dabei steht jedoch zunächst lediglich eine grobe Aufteilung der strategischen Geschäftseinheit Firmenkunden nach dem Firmenumsatz im Vordergrund. Diese orientiert sich - wie in Tabelle 1 dargestellt - an der Einteilung der Ratingklassen im Kontext des BVR-II-Rating ${ }^{\prime S}$.

\begin{tabular}{|l|c|}
\hline \multicolumn{1}{|c|}{ Segment } & Umsatz \\
\hline Gewerbekunden / Freiberufler & bis 350 T€ \\
\hline Mittelstand & bis 5.000 T€ \\
\hline Oberer Mittelstand & bis 1 Mrd. $€$ \\
\hline Große Firmenkunden & über 1 Mrd. $€$ \\
\hline
\end{tabular}

Tabelle 1: Segmentzuordnung in der Firmenkundenbetreuung gem. BVR ${ }^{16}$

Als mögliche Erweiterung dieser groben Klassifizierung wird die zusätzliche Aufnahme der Merkmale Aktivvolumen und Deckungsbeitrag vorgeschlagen. ${ }^{17}$ Der $B V R$ fordert zudem eine darüber hinausgehende Informationsveredelung und eine segmentspezifische Ausgestaltung der Beratungs- und Betreuungsleistung. ${ }^{18}$ Dieser kundenorientierten Segmentierungsausrichtung wird in der vorliegenden Arbeit gefolgt. Der Zuschnitt des zu entwickelnden Segmentierungsansatzes erfolgt insofern unter Berücksichtigung sämtlicher bankengruppenspezifischer Anforderungen. Deren

\footnotetext{
Vgl. Krüger, Markus (2002), S. 10

Vgl. BVR (2001), S. $11 \mathrm{ff}$.

Es handelt sich hierbei um ein bankinternes Rating-Verfahren zu Bonitätseinstufung gewerblicher Kreditnehmer. Vgl. für einen Überblick beispielsweise Klingbeil, Ernst / Yousefian, Patrick (2002), S. 28 f. oder Nowak, Helge (2002), S. 22 ff.

Vgl. BVR (2004), S. 1

Vgl. BVR (2004), S. $6 \mathrm{f}$.

Vgl. BVR (2004), S. $14 \mathrm{f}$.
} 
Operationalisierung soll dabei auf der verbandsseitig vorgeschlagenen Grobsegmentierung aufsetzen.

Das Kernstück der Vertriebs- und Betreuungskonzeption im Firmenkundengeschäft der Genossenschaftsbanken stellt der VR-Finanzplan Mittelstand dar. ${ }^{19}$ Es handelt sich dabei um einen Marktbearbeitungsansatz, der die ganzheitliche, bedarfsorientierte und strukturierte Beratung in den Mittelpunkt der Kundeninteraktion stellt. Der Fokus liegt dabei auf einer qualitativen Wettbewerbsdifferenzierung und einer Verbesserung der Marktdurchdringung. ${ }^{20}$ Abbildung 2 veranschaulicht die strategische Stoßrichtung des Konzeptes:

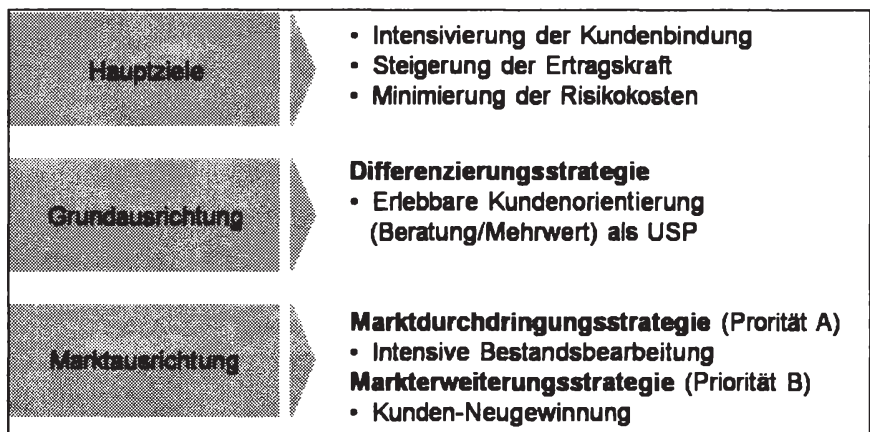

Abbildung 2: Strategische StoBrichtung des VR-Finanzplan Mittelstand ${ }^{21}$

Im weiteren Verlauf dieser Arbeit werden unterschiedliche Elemente des $V R$-Finanzplan Mittelstand aufgegriffen, teilweise vertieft und in die konzeptionellen Überlegungen einbezogen.

19 Vgl. Dehne, Thorsten / Zimmermann, Yvonne, S. 18 existiert eine weitere, sekundäre Zielrichtung, die aber nicht im Fokus dieser Arbeit steht und daher hier nicht vertiefend behandelt wird. 


\section{Firmenkundengeschäft im Wandel}

\subsection{Wettbewerbsposition genossenschaftlicher Kreditinstitute}

Sowohl die deutschen Kreditinstitute als auch deren Kunden erleben derzeit einen tiefgreifenden, anhaltenden Wandel. Als Auslöser dieses Veränderungsprozesses können beispielhaft die Globalisierung oder die rasante Entwicklung der Informations- und Kommunikationstechnologie angeführt werden. ${ }^{22}$

Um die Positionierung der Genossenschaftsbanken transparent zu machen, soll nach einer knappen Darstellung der Struktur des deutschen Bankensektors auf die Wettbewerbssituation im strategischen Geschäftsfeld Firmenkunden eingegangen werden.

\subsubsection{Marktstruktur}

Die deutschen Genossenschaftsbanken sind den Universalbanken ${ }^{23}$ zuzuordnen. Neben den Kreditgenossenschaften gehören auch die öffentlich-rechtlichen Sparkassen und die Kreditbanken dieser Gruppe an.

Analog zur genossenschaftlichen Organisation sind auch die Sparkassen in einem Finanzverbund organisiert. ${ }^{24}$ Das wesentliche Unterscheidungsmerkmal ist in der Trägerschaft der beiden Institutsgruppen zu sehen. Während die Sparkassen und Landesbanken (mehrheitlich) in öffentlichem Besitz stehen, sind die Kreditgenossenschaften Eigentum ihrer Mitglieder (i.d.R. Privatpersonen und Unternehmen) und somit der Privatwirtschaft zuzuordnen.

Zu den Kreditbanken gehören die Großbanken (Deutsche Bank AG, Dresdner Bank $A G$, Commerzbank $A G$, HypoVereinsbank $A G$, Postbank $A G$ ), die Regional- und Privatbanken, sowie Zweigniederlassungen ausländischer Kreditinstitute.

22 Vgl. Steffens, Udo (2002), S. 79

23 Universalbanken führen die Mehrzahl der in \$1 KWG aufgeführten Bankgeschäfte durch. Neben den Universalkreditinstituten existieren noch Spezialkreditinstitute, welche sich auf einzelne der im Gesetz genannten Bereiche fokussieren; vgl. Hartmann-Wendels, Thomas et al. (2007), S. 30. Das Firmenkundengeschäft gehört bei den Spezialinstituten nicht zum Kerngeschäft, daher bleiben diese im weiteren Verlauf der Arbeit unberücksichtigt. Dieser gliedert sich - wie auch in der genossenschaftlichen Bankengruppe - in regional orientierte Primärinstitute mit direktem Kontakt zum Endkunden (Privatkunde, Firmenkunde, etc.), in die Primärbanken unterstützende Spitzeninstitute und diverse Verbundunternehmen, welche banknahe Finanzdienstleistungen anbieten. Zum Ende des Jahres 2005 zählte der Sparkassensektor 463 Primärinstitute mit über 16.000 Geschäftsstellen, vgl. DSGV (2006a). Als Spitzeninstitute fungierten elf Landesbanken, vgl. DSGV (2006b). 
Die Großbanken sind - unter Einbindung von Tochtergesellschaften und anderweitiger Kooperationspartner - ebenfalls mit einem umfassenden Portfolio an Finanzdienstleistungen am Markt vertreten und i.d.R. auch international aktiv. Die Regional- und Privatbanken haben sich hingegen zumeist auf komplexe Beratungsdienstleistungen (Mergers \& Acquisitions, Vermögensverwaltung, Auslandsgeschäft, etc. $)^{25}$ spezialisiert.

\subsubsection{Wettbewerbssituation im Firmenkundengeschäft}

Die Analyse der Wettbewerbsstruktur orientiert sich an der Methodik von PORTER, nach dem die fünf Wettbewerbskräfte

- Rivalität unter den vorhandenen Wettbewerbern

- Markteintritt neuer Konkurrenten

- Gefahr durch Ersatzdienstleistungen

- Verhandlungsstärke der Abnehmer

- Verhandlungsstärke der Lieferanten

die Branchenrentabilität bestimmen. ${ }^{26}$ Die genaue Kenntnis dieser Wettbewerbsregeln bildet die Basis zur Entwicklung einer Wettbewerbsstrategie und ist Grundlage der Erringung von Wettbewerbsvorteilen.

\section{Rivalität}

Das Engagement der genannten Bankengruppen im Firmenkundengeschäft differiert. So fühlen sich die genossenschaftlichen Kreditinstitute in besonderer Weise dem Mittelstand verpflichtet ${ }^{27}$. Auch die Sparkassen haben in der Firmenkundenbank schwerpunktmäßig mittelständische Unternehmen im Fokus. ${ }^{28}$ Im Gegensatz dazu ist das Geschäft der Groß- und Privatbanken, sowie der ausländischen Kreditinstitute traditionell auf Großkunden und Konzerne ausgerichtet. Hier zeichnet sich allerdings seit geraumer Zeit ein Strategiewechsel in der Weise ab, dass der Mittelstand zunehmend als attraktives Marktsegment wiederentdeckt wird. ${ }^{29}$

\footnotetext{
Vgl. Strauß, Marc-R. (2006), S. 24

26 Vgl. Porter, Michael E. (2000), S. 29

27 Vgl. BVR (2006a). Zur Abgrenzung des Mittelstandsbegriffs sollen in dieser Arbeit die quantitative Abgrenzung des IfM Bonn in Anhang 1, sowie die Ausführungen von Günterberg, Brigitte / Wolters, Hans-Jürgen (2002) herangezogen werden.

29 Vgl. u.a. Bastian, Nicole / Müller, Oliver (2005), S.17, Köhler, Peter / Potthoff, Christian (2005), S. 29 oder o.V. (2005), S. 4
} 


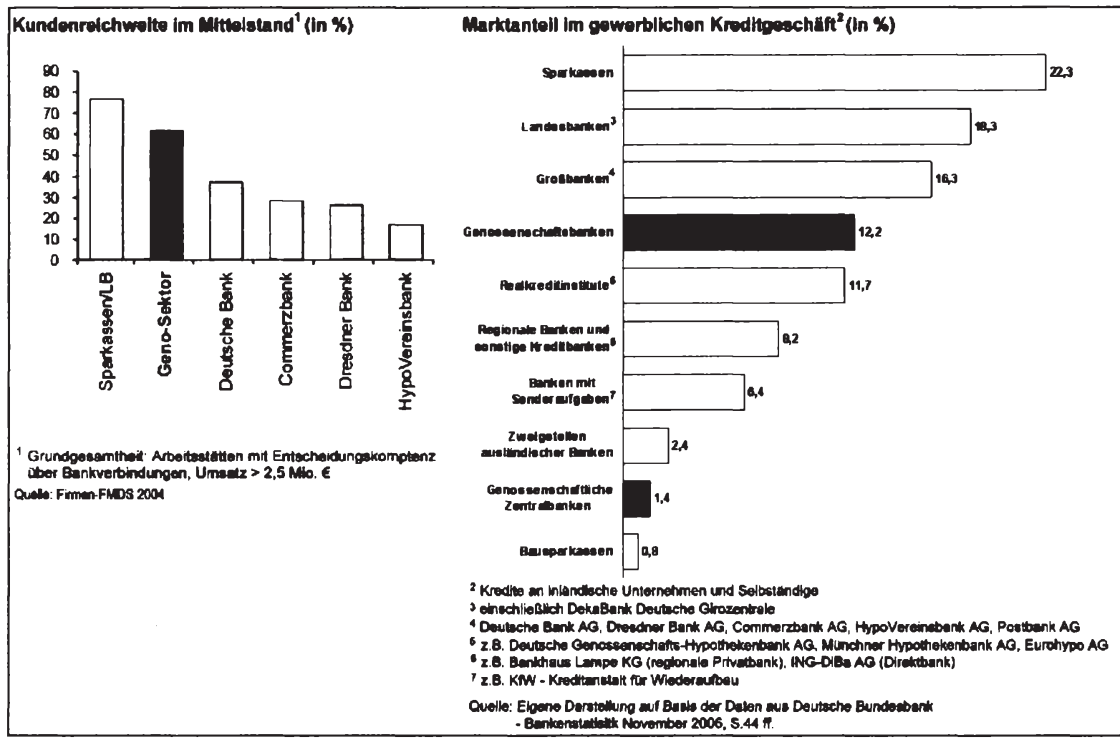

Abbildung 3: Kundenreichweite und Marktanteile im deutschen Firmenkundengeschäft

Wie Abbildung 3 beispielhaft am gewerblichen Kreditgeschäft zeigt liegt der Marktanteil der Kreditgenossenschaften inklusive der Spitzeninstitute per November 2006 bei $13,6 \%$. Bei einer Kundenreichweite von knapp über $60 \%$ impliziert dies eine unzureichende Marktdurchdringung. Es sei jedoch angemerkt, dass die Genossenschaftsbanken z.B. im Bereich der Langfristdarlehen oft als Vermittler innerhalb des Finanzverbundes (z.B. an Hypothekenbanken, wie die Münchener Hypothekenbank AG) agieren. Insofern kann davon ausgegangen werden, dass es sich bei Teilen der Positionen „Realkreditinstitute“ und „Banken mit Sonderaufgaben“ (u.a. Vermittlung von Förderkrediten), um durch Kreditgenossenschaften vermitteltes Geschäftsvolumen handelt.

Aufgrund der homogenen Strukturen und der regionalen Dichte können die Sparkassen als Hauptwettbewerber der genossenschaftlichen Institute identifiziert werden. Im mittelständischen Firmenkundengeschäft gilt es zudem dem verstärkten Wettbewerb durch Großbanken und ausländischen Kreditinstitute zu begegnen. Insgesamt ist die Wettbewerbsintensität jedoch als gemäßigt zu bezeichnen, zukünftig muss jedoch - u.a. aufgrund des fortwährenden Konzentrationsprozesses - mit einer Rivalitätsverschärfung gerechnet werden. ${ }^{30}$

$30 \quad$ Vgl. Steffens, Udo (2002), S. 89 


\section{Markteintritt neuer Konkurrenten}

Da die Markteintrittsbarrieren im Bankensektor aufgrund bankaufsichtsrechtlicher Bestimmungen oder auch aufgrund von economies of scale ${ }^{31}$ und learning-curve effects $^{32}$ als relativ hoch zu bezeichnen sind, konzentriert sich die Gefahr potenzieller Neuanbieter im wesentlichen auf den Markteintritt ausländischer Kreditinstitute und Finanzkonzerne. ${ }^{33}$ Es ist wahrscheinlich, dass ein solcher Einstieg in das Firmenkundengeschäft über Akquisitionen oder Fusionen verläuft. ${ }^{34}$ Die Bedrohung ist dabei nicht auf die Genossenschaftsbanken beschränkt, sondern erfasst auch deren Verbundpartner. So bietet beispielsweise die Fortis Bank ${ }^{35}$ Forderungsmanagement-Lösungen an und tritt somit u.a. in Konkurrenz zur VR FACTOREM GmbH ${ }^{\beta 6}$.

Eine weitaus größere Gefahr für regional operierende Banken stellt die bereits beschriebene Neuorientierung der Groß- und Privatbanken dar. Gegen diese wirkt die Kundenbindung als entscheidende Eintrittsbarriere in den Regionalmarkt. Besonders im beratungsintensiven mittelständischen Firmenkundengeschäft besteht häufig eine enge Bindung an die Hausbank. ${ }^{37}$

In der Firmenkundenbetreuung darf neben der betrieblichen auch die private Sphäre (Privatsituation des Unternehmers) nicht außer Acht gelassen werden. ${ }^{38}$ Im Privatkundengeschäft sind in den letzten Jahren vor allem Automobil- und Direktbanken als Mitbewerber hinzugekommen. ${ }^{39}$ In diesem Zusammenhang wird sich zeigen, dass die ganzheitliche Betreuung einen entscheidenden Ansatz darstellt, um sich gezielt von derartigen Konkurrenzinstituten abzugrenzen. ${ }^{40}$

\section{Gefahr durch Ersatzdienstleistungen}

Als Substitute von Finanzdienstleistungen sind solche branchenfremden Dienstleistungen zu verstehen, die die gleichen Bedürfnisse befriedigen, wie die brancheneigenen. ${ }^{4}$ Die Substituierbarkeit ist aufgrund der Verbundorganisation lediglich als periphere Bedrohung für die Kreditgenossenschaften einzuschätzen. So werden z.B. innovative Finanzierungsformen, wie Beteiligungsfinanzierungen über MezzanineKapital als Alternative zur herkömmlichen Unternehmensfinanzierung über den

${ }^{31}$ Betriebsgrößenvorteile, z.B. Fixkostendegression; vgl. u.a. Meffert, Heribert (2000), S. 250

32 Erfahrungskurveneffekte, durch die gezielte Nutzung von gesammelten Erfahrungen zur Kostenreduktion; vgl. Meffert, Heribert (2000), S. 251

33 Vgl. Börner, Christoph J. (2005), S. 57

Vgl. Steffens, Udo (2002), S. 85

Fortis Bank (2006)

Leistungsangebot u.a. Factoring und Debitorenmanagement; vgl. VR FACTOREM GmbH (2006)

Zu den Erfolgsfaktoren einer engen Kundenbindung vgl. Kapitel 4.2.

Vgl. u.a. BVR (2006e), S. 13

Vgl. Paul, Stephan (2002), S. 33

Vgl. Kapitel 5.1.1

Die Ausführungen von PORTER wurden hier auf den Banksektor übertragen; vgl. Porter, Michael E. (1999), S. 56 f. 
genossenschaftlichen Finanzverbund angeboten ${ }^{42}$ Insofern wird eine Abschirmung vor Substitutionen dadurch erreicht, dass der Verbund durch eine Erweiterung des Leistungsportfolios selbst als Anbieter auftritt. Dies hat jedoch einen verbundinternen Wettbewerb zur Folge.

\section{Verhandlungsstärke der Abnehmer}

Die Verhandlungsmacht der Firmenkunden hat sich in den letzten Jahren deutlich erhöht, was u.a. in rückläufigen Zinsmargen zum Ausdruck kommt (vgl. Abbildung 4). ${ }^{43}$ Ursachen sind beispielsweise in einer erhöhten Marktransparenz, einem verändertem Preis- und Qualitätsbewusstsein und einer veränderten Anforderungsstruktur ${ }^{44} \mathrm{zu}$ suchen.

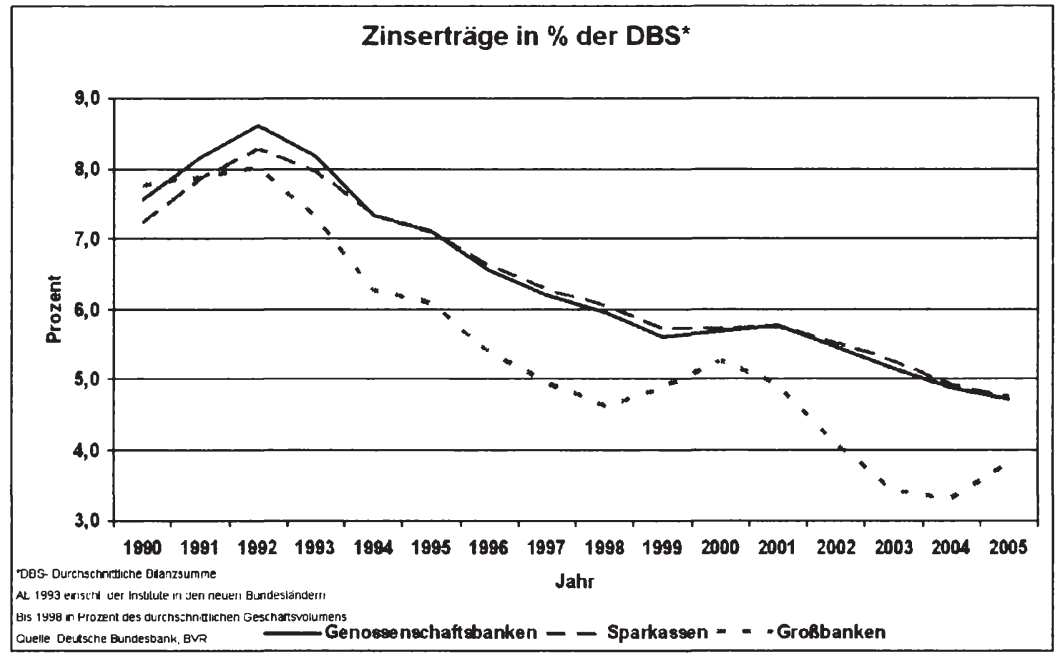

Abbildung 4: Entwicklung der Zinsmargen

\footnotetext{
42 Beteiligungsfinanzierungen werden im Verbund z.B. über die VR-Mittelstandskapital Unternehmensbeteiligungs $A G$ offeriert.

$43 \mathrm{Vgl}$. Schmoll, Anton (2006), S. 16

44 Im Rahmen der empirischen Analyse in Kapitel 8, wird die Anforderungsstruktur im Firmenkundengeschäft unter Einsatz multivariater Analyseverfahren untersucht.
} 


\section{Verhandlungsstärke der Lieferanten}

Der Wettbewerbsfaktor Verhandlungsstärke der Lieferanten ist auf das Dienstleistungsportfolio bezogen als gering einzuschätzen, da Finanzdienstleistungen i.d.R. im Rahmen von verbund- bzw. konzerninternen Kooperationen angeboten werden. STEFFENS sieht die Lieferantenmacht im Bankenbereich daher vornehmlich bei den Mitarbeitern, die in konjunkturellen Hochphasen, Knappheitsgehälter realisieren können. ${ }^{45}$

\subsubsection{Kundenstruktur im Firmenkundengeschäft}

$\mathrm{Da}$ in dieser Arbeit die genossenschaftlichen Kreditinstitute im Fokus der Betrachtung stehen, beziehen sich die weiteren Ausführungen auf die wesentliche Kundengruppe, die kleinen und mittleren Unternehmen (KMU). ${ }^{46}$

Die Kundenstruktur im Firmenkundengeschäft von Banken ist als äußerst heterogen zu bezeichnen. Die Verschiedenheit ist u.a. durch unterschiedliche Unternehmensgröße, Branchenzugehörigkeit und Bedarfslage gekennzeichnet. ${ }^{47}$ Anhang 2 zeigt die beispielhafte Verteilung nach Sektoren im Firmenkundengeschäft von Genossenschaftsbanken.

Im Folgenden sollen kurz Veränderungsprozesse skizziert werden, mit denen eine Vielzahl der Firmenkunden konfrontiert sind, um daraus Implikationen für das Bankgeschäft abzuleiten.

In vielen Branchen eröffnen sich durch die Globalisierung neue Absatzmärkte. Dies bedeutet auf der einen Seite zusätzliches Ertragspotenzial, auf der anderen Seite jedoch auch einen verschärften internationalen Wettbewerb. ${ }^{48}$ Durch die EU-Osterweiterung ergaben sich - neben der Vergrößerung der Marktdimension - für den Mittelstand erstmals Möglichkeiten Kostenvorteile in einem internationalen Rahmen durch Outsourcing oder Offshoring zu erzielen ${ }^{49}$ Eine Gefahr birgt in diesem Zusammenhang jedoch der Wegzug von Großkunden. Abbildung 5 zeigt die Triebkräfte der Internationalisierung des Mittelstandes und mögliche Reaktionen:

45 Steffens, Udo (2002), S. 88

46 Zur Abgrenzung des Mittelstandsbegriffs sollen in dieser Arbeit die quantitative Abgrenzung des IfM Bonn in Anhang 1, sowie die Ausführungen von Günterberg, Brigitte / Wolters, Hans-Jürgen (2002) herangezogen werden.

48 Vgl. Schmoll, Anton (2006), S. 13

49 Vgl. Brenken, Anke (2006), S. 10 f.; „Die Begriffe Outsourcing und Offshoring werden uneinheitlich verwendet." Im Rahmen dieser Arbeit soll unter Outsourcing „die Aufgabe der eigenen Herstellung von Vorprodukten zugunsten des Kaufs bei Zulieferern“" und unter Offshoring „die Verlagerung der Produktion in firmeneigene Niederlassungen, die im Ausland errichtet wurden“, verstanden werden; Brenken, Anke (2006), S. 10 


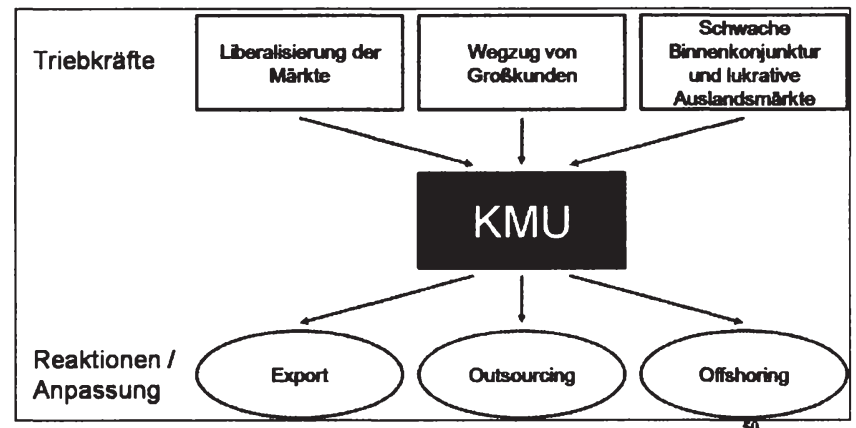

Abbildung 5: Triebkräfte der Internationalisierung des Mittelstandes

Firmenkunden sind zudem oft gezwungen mit dem technologischen Wandel Schritt zu halten, um die Anforderungen ihrer Kunden oder auch Qualitätsnormen zu erfüllen. ${ }^{51}$ Des Weiteren werden Unternehmen im Rahmen der Kreditfinanzierung aufgrund der Vorgaben von Basel II ${ }^{2}$ zu einem aktiven und offenen Ratingdialog angehalten. ${ }^{53}$

Um diese Herausforderungen bewältigen zu können, entstehen auch neue Anforderungen gegenüber dem Bankensektor. ${ }^{54}$ So erhöht sich mit zunehmender Komplexität auch der Anspruch an die Zusammenarbeit. Die Unternehmen sind auf der Suche nach professionellen Partnern, die sie auf Ihrem Weg unterstützen. Für das Firmenkundengeschäft ergeben sich aus dieser Situation neue Chancen, wenn es gelingt die Betreuung auf die Unternehmensgröße, Branchenzughörigkeit und Anforderungsstruktur passgenau abzustimmen. ${ }^{55}$ Dabei stellt die Intensivierung des Kundenbindungsmanagements einen entscheidenden Erfolgsfaktor dar. ${ }^{56}$

So Vgl. Brenken, Anke (2006), S. 7

Vgl. Schmoll, Anton (2006), S. 13

52 Bei Basel II handelt es sich um Kapitaladäquanzrichtlinie, deren zentrales Element eine nach Risiko gestaffelte Eigenkapitalunterlegung bei der Kreditvergabe von Banken ist. Für weitere Erläuterungen vgl. u.a. Taistra, Gregor (2003), S. 1 f.

Vgl. Taistra, Gregor (2004), S. $18 \mathrm{ff}$.

Zur Strukturierung dieser Anforderungen, vgl. Kapitel 4.2.2.

Zur Ausprägung der Kundenanforderungen und den Implikationen für das Leistungsportfolio vgl. die empirische Analyse in Kapitel 8 und die Optimierungsvorschläge in Kapitel 9 


\subsubsection{SWOT-Analyse ${ }^{57}$ für das genossenschaftliche Firmenkundengeschäft}

Die vorhergehenden Ausführungen werden nun zusammengefasst und um einige weitere Aspekte ergänzt, um im Rahmen einer SWOT-Analyse die spezifische Ausgangssituation für das Firmenkundengeschäft der genossenschaftlichen Bankengruppe herauszustellen. Dabei wurden diejenigen Chancen und Risiken, welche in der vorliegenden Arbeit genauer betrachtet bzw. durch ihre Ergebnisse direkt oder indirekt beeinflusst werden, im Schriftschnitt ,kursiv" hervorgehoben und mit einem Querverweis versehen.

\begin{tabular}{|c|c|}
\hline $\begin{array}{l}\text { Starken } \\
\text { Regionale Năhe, Markt und Kundenkenntnis } \\
\text { Markt- und wettbewerbsfähige Leistungsportfolio } \\
\text { durch genossenschaftlichen Finanz Verbund } \\
\text { Imagevorteile gegenüber Mitbewerbem }\end{array}$ & $\begin{array}{l}\text { Schwăchen } \\
\text { Unterentwickelte Vertriebskultur, -ausrichtung und - } \\
\text { steuerung } \\
\text { Schwache Potenzialausschöpfung, } \\
\text { z.B. ungenügendes Cross-Selling } \\
\text { Zu starke Kapazitătsbindung in } \\
\text { potenzialschwachen Segmenten } \\
\text { Unzureichende Risikoprävention } \\
\text { Fokussierung auf Kreditrisiken erschwert aktives } \\
\text { Neugeschaft }\end{array}$ \\
\hline $\begin{array}{l}\text { Chancen } \\
\text { Vertriebliche Nutzung des } \\
\text { Firmenkundenpotenzials } \\
\rightarrow \quad \text { Kundensegmentierung als Basis einer } \\
\text { verbesserten Potenzlalausschópfung } \\
\text { (s. Kapltel 7) } \\
\text { Unternehmer auch als Privatkunden beraten } \\
\rightarrow \quad \text { Ganzheltiche FIrmenkundenbetreuung } \\
\text { (s. Kapltel 5) } \\
\text { Nutzung der Kundenreichweite zur } \\
\text { Geschăftsintensivierung }\end{array}$ & 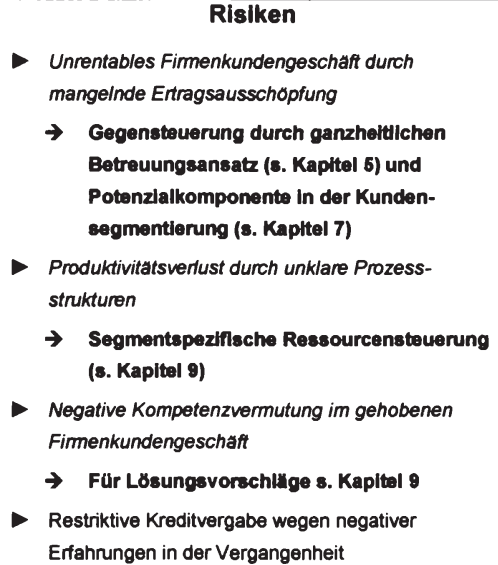 \\
\hline
\end{tabular}

Abbildung 6: SWOT-Analyse für das genossenschaftliche Firmenkundengeschăft ${ }^{58}$

57 Die SWOT-Analyse (Strenghts, Weaknesses, Opportunities, Threats) ist ein strategisches Instrument zur Untersuchung von Stärken, Schwächen, Chancen und Risiken einer Unternehmung, vgl. Bruhn, Manfred / Meffert, Heribert (2006), S. 167 Zusammengestellt und angepasst aus Kapitel 3.1.1 bis 3.1.3; sowie Käser, Burkhard et al. (2004), S. 3 f. und S. 27; BVR (2006e), S. $10 \mathrm{ff}$. 


\subsection{Notwendigkeit zur Differenzierung im Wettbewerb}

\subsubsection{Homogenität von Bankprodukten ${ }^{59}$}

Das Angebot an Bankprodukten im Firmenkundengeschäft ist von einer starken Homogenität und einer leichten Imitierbarkeit geprägt. ${ }^{(0)}$ Aufgrund der AllfinanzAusrichtung der in Kapitel 3.1.1 genannten Bankengruppen ist selbst bei komplexeren, neuartigen Produkten eine verhältnismäßig einfache Nachahmung möglich. Kann ein Konkurrenzinstitut selbst keine Alternativleistung anbieten, wird die Imitation i.d.R. auf Verbund- bzw. Konzernebene erfolgen.

Der Wert, den der Kunde solchen Kernleistungen zuschreibt, resultiert aus der Wahrnehmung des Preises und der Qualität. ${ }^{61}$ Diese beiden Faktoren bilden die zentralen Elemente der PORTERschen Wettbewerbsstrategien. Dabei wird zwischen der Umfassenden Kostenführerschaft, der Differenzierung und der Konzentration mit Kostenoder Differenzierungsschwerpunkt unterschieden.

Während die Strategie der Kostenführerschaft einen umfassenden Kostenvorsprung innerhalb einer Branche intendiert, welcher sich in günstigen Preisen für den Kunden niederschlägt, kann die Erschaffung eines einzigartigen Angebotes, dass auf einer dem Wettbewerb überlegenen Qualiätsposition beruht, als Ziel der Differenzierungsstrategie verstanden werden. ${ }^{62}$ MEFFERT weist in diesem Zusammenhang darauf hin, dass die überlegene Qualitätsposition als „mehrdimensionales Optimierungsproblem“ zu interpretieren ist und, dass die Qualitätsbeurteilung „von der Erwartungshaltung, der tatsächlich (subjektiv) erlebten Leistung, von situativen Faktoren, sowie durch den Vergleich mit Konkurrenzprodukten" beeinflusst wird. ${ }^{63}$

Implizierte die am Anfang des Abschnitts dargestellte Gleichartigkeit von Bankleistungen ein geringes Differenzierungspotenzial, entsteht bei Betrachtung des Leistungserstellungsprozesses und unter Berücksichtigung von Mehrwertdienstleistungen, so genannten Value Added Services, ein anderes Bild. ${ }^{64}$ So spielen im

59 Folgt man einer engen Definition des Dienstleistungsbegriffs, so handelt es sich bei nahezu allen Bankleistungen um Dienstleistungen, da sie folgende Besonderheiten aufweisen: Die Erforderlichkeit der Leistungsfähigkeit des Anbieters, die Integration eines externen Faktors und die Immaterialität des Leistungsergebnisses; vgl. Bruhn, Manfred / Meffert, Heribert (2006), S. 60 ff.. Sowohl in der Bankpraxis als auch in der Literatur, findet der Produktbegriff in Zusammenhang mit Bankleistungen noch häufig Verwendung. In dieser Arbeit soll vereinfachend von Produkten gesprochen werden, wenn es sich um Kernleistungen (Core Services) im Bankvertrieb - wie z.B. die Geldanlage in Festgeldern oder die Vergabe von Investitionskrediten - handelt; zum Begriff Kernleistung vgl. Bruhn, Manfred / Meffert, Heribert (2006), S. 360 f.. Vgl. Börner, Christoph J. (2005), S. 54, sowie Schlosser, Christoph (2004), S. 29

Vgl. Hinterhuber, Hans H. et al. (2006), S. $16 \mathrm{ff}$.. Zur Erläuterung des Qualitätsbegriffs wird die Definition gem. DIN EN ISO 8402 herangezogen: „Die Gesamtheit von Merkmalen einer Einheit bezüglich ihrer Eignung, festgelegte und vorausgesetzte Erfordernisse zu erfüllen.“ Vgl. Porter, Michael E. (1999), S. 71 ff.; sowie die grafische Darstellung in Anhang 3 Meffert, Heribert (2005), S. 155 Vgl. Büschgen, Anja / Büschgen, Hans E. (2002), S. 99 f. 
Beratungsgespräch Faktoren wie etwa eine vertrauensgeprägte Geschäftsbeziehung, eine hohe Fach- und Erläuterungskompetenz des Firmenkundenbetreuers, sowie Freundlichkeit eine wichtige Rolle und bieten vielfältige Differenzierungsansätze. ${ }^{65}$ Zusatzdienstleistungen wie z.B. ein jährliches Rating- und Bilanzanalysegespräch stellen weitere Abgrenzungsmöglichkeiten dar.

Es ist zu beobachten, dass die Differenzierungsstrategie im Firmenkundengeschäft absolut dominierend ist. Ein umfassender Preiswettbewerb hat faktisch keine Bedeutung, ${ }^{66}$ sehr wohl wird jedoch mit „Lockangeboten“ gearbeitet um einen Eintritt in den Regionalmarkt zu verschaffen oder Marktanteile zu erhöhen. ${ }^{67}$ Der Differenzierungsschwerpunkt ist dabei jedoch sehr unterschiedlich, wie das Positionierungsmodell in Abbildung 7 zeigt:

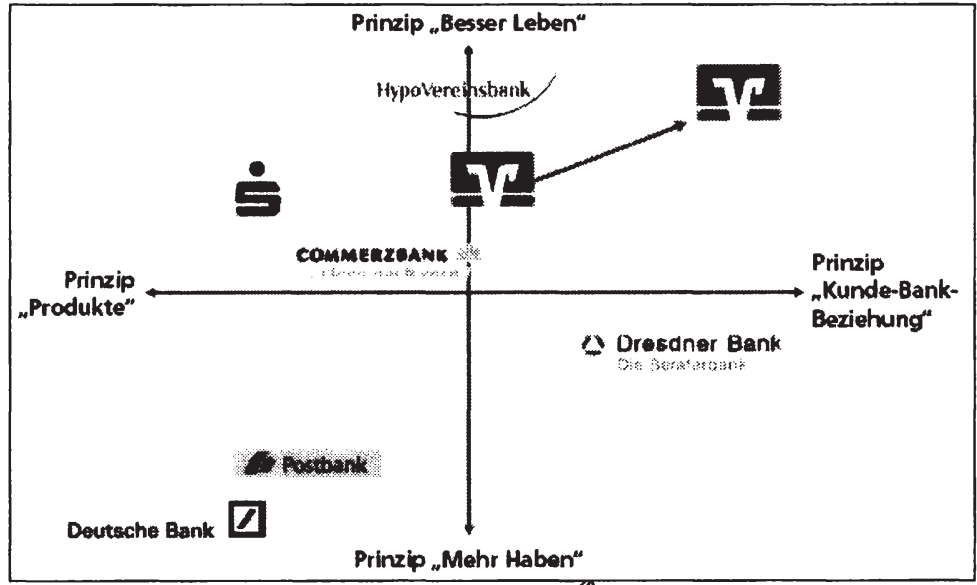

Abbildung 7: Positionierungsmodell deutscher Banken6

Der Genossenschaftssektor unternimmt derzeit den Versuch einer Weiterentwicklung der Differenzierungsstrategie und konzentriert sich dabei schwerpunktmäßig auf den Aspekt Kunde-Bank-Beziehung. Dieser kundenorientierte Ansatz ist - wie bereits weiter oben erwähnt - auch in der strategischen Ausrichtung der genossenschaftlichen Bankengruppe aufzufinden.

65 Als Ursachen können hier z.B. die Abstraktheit und die Immaterialität der angebotenen Leistungen angeführt werden; vgl. hierzu Börner, Christoph J. (2005), S. 54 f.. Zur Wirkung der genannten Attribute auf die Kundenzufriedenheit und die Kundenbindung, s. Kapitel 8

Im Firmenkundengeschäft kann davon ausgegangen werden, dass der Preis im Regelfall nur einer von mehreren entscheidenden Faktoren ist; vgl. u.a. Büschgen, Hans E. (2000), S. 592.

So werden im Kreditgeschäft bei großen Finanzierungsprojekten häufig bewusst Mindermargen in Kauf genommen, was mit der Hoffnung verbunden ist, diese im weiteren Verlauf der Geschäftsbeziehung durch Folgegeschäfte wieder auszugleichen.

Bookhagen, Bettina (2006), S. 58 


\subsubsection{Abwanderungsanalyse im Firmenkundengeschäft}

Obwohl empirische Untersuchungen gezeigt haben, dass es je nach Branche das 5 bis 15 -fache mehr kostet einen neuen Kunden zu akquirieren, als einen bestehenden Kunden zu halten, ist vielfach zu beobachten, dass der strategische Stellenwert der Kundenbindung noch unterschätzt wird. ${ }^{69}$ So lieferte eine branchenübergreifende Unternehmensbefragung für die teilnehmenden Finanzdienstleister das folgende, alarmierende Ergebnis:

- 92,3\% kennen die Kundenakquisitionskosten nicht,

- 94,9\% sind die Kundenbindungskosten nicht bekannt,

- $100 \%$ der Befragten können keine Aussage zu den Kundenrückgewinnungskosten treffen. $^{70}$

Diese Zahlen sind jedoch kein Beleg dafür, dass Banken gar kein Churn Management betreiben. Es ist vielmehr festzustellen, dass der Grad der Systematisierung und die Intensität der Aktivitäten differieren. Als Grund kann - neben der teilweise zu geringen strategischen Verankerung - das Fehlen oder die suboptimale Nutzung notwendiger Softwareunterstützungen genannt werden." Eine Studie der Unternehmensberatung Bain \& Company im Privatkundengeschäft europäischer Banken dokumentiert, dass eine gute Performance bei Wachstumshebeln wie dem „Management kritischer Kundeninteraktionen“ und einer „Frühzeitigen Neukundenbindung“, zu einer niedrigen Abwanderungsrate und überdurchschnittlichen wirtschaftlichen Ergebnissen führt. ${ }^{72}$

Zusammenfassend ist zu konstatieren, dass aufgrund der Homogenität der Kernleistungen, sowie der Zeit- und Kostenintensität von Akquisitions- und Wiedergewinnungsmaßnahmen, die Differenzierung über eine Kundenbindungsstrategie einen entscheidenden Erfolgsfaktor im Firmenkundengeschäft darstellt.

69 Vgl. Werani, Thomas (2006), S. 154, sowie Stahl, Heinz K. (2006), S. 92. STAHL stellt zudem am Beispiel des Kreditkartengeschäftes fest, dass eine zweiprozentige Verringerung der Abwanderungsquote, eine zehn-prozentige Senkung der kundenbezogenen Kosten entspricht. 


\section{Kundenbindungsmanagement als Differenzierungsmerkmal}

Die Ausführungen in Kapitel 3.2 zeigen, dass es aufgrund der Dominanz von Differenzierungsstrategien im Firmenkundengeschäft äußerst schwierig ist, eine erkennbare Abgrenzung vom Wettbewerb zu erreichen. Es bedarf hierzu einer klaren strategischen Positionierung, über deren operative Implementierung ein nachhaltiger Wettbewerbsvorteil erzeugt werden muss.

Die Genossenschaftsbanken konzentrieren sich dabei auf die Kundenbindung, die durch den Aufbau einer einzigartigen Kunde-Bank-Beziehung gestärkt bzw. intensiviert werden soll. ${ }^{73}$

Im Folgenden wird nun zunächst die strategische Wichtigkeit der Kundenbindung erörtert, um danach die wesentlichen Determinanten einer intensiven Kundenbindung zu identifizieren. Zum Abschluss des Kapitels soll, nach einer kurzen begrifflichen Abgrenzung, gezeigt werden, wie durch ein integriertes Kundenbindungsmanagement ein spürbarer Mehrwert für den Firmenkunden entsteht.

\subsection{Strategische Bedeutung der Kundenbindung}

Da der Begriff der Kundenbindung in der Literatur unterschiedlich verwendet wird, soll zunächst eine Definition gefunden werden, die den Beziehungsaspekt in den Mittelpunkt stellt und auf der die nachfolgenden strategischen Überlegungen aufsetzen können:

Kundenbindung soll demnach in der vorliegenden Arbeit als psychologischer Zustand verstanden werden, der den Grad der Bereitschaft zur Fortsetzung bzw. Intensivierung einer Geschäftsbeziehung angibt. ${ }^{74}$

Die langfristige Kundenbindung hat insbesondere auf engen Märkten mit niedrigen Wachstumsraten, zu denen auch das mittelständische Firmenkundengeschäft zählt, an Bedeutung gewonnen. ${ }^{75}$ Betrachtet man lediglich die Ersttransaktion, so ist der Ertragswert des Kunden i.d.R. noch relativ gering. Erst durch Etablierung einer stabilen Geschäftsbeziehung besteht die Möglichkeit das Ertragspotenzial voll auszuschöpfen. ${ }^{76}$ Existiert im Rahmen einer solchen Geschäftsbeziehung zusätzlich ein

73 Zur Verankerung der Kundenorientierung in der strategischen Ausrichtung vgl. Kapitel 2.2.

Definition in Anlehnung an den Commitment-Begriff, vgl. hierzu z.B. Desphandé, Rohit et al. (1992), S. 316; Morgan, Robert M. / Hunt, Shelby D. (1994), S. 23 oder Söllner, Albrecht (1999), S. 219 ff.. Dem sonst auch hăufig verwendeten Konzeptualisierungsansatz über die Komponenten „Faktisches Verhalten“ und „Verhaltensabsicht" wird hier nicht gefolgt, da dieser streng genommen Ursache (Bindungszustand) und Wirkung (Verhalten / Verhaltensabsicht) gleichsetzt; vgl. zu diesem Ansatz beispielsweise Diller, Hermann (1996), S. 83 oder Bruhn, Manfred (2003), S.104; sowie zur Kritik Eggert, Andreas (2000), S. 121

76 Vgl. Meffert, Heribert (2005), S. 148 
besonderes Vertrauensverhältnis ${ }^{77}$, können aufgrund eines intensiven Austausches proprietäre Informationsvorteile entstehen, welche im Wettbewerb nutzbar sind und zu einer verbesserten (Risiko-)Einschätzung des Kunden führen. Außerdem bietet eine enge Kundenbindung die Möglichkeit intertemporaler Ertragsverschiebungen, welche auf Ebene einer Einzeltransaktion einen erhöhten Spielraum in der Konditionsgestaltung und somit eine verbesserte Wettbewerbsposition zur Folge haben. ${ }^{78}$

Wirtschaftlich betrachtet kann eine starke Kundenbindung zu einer erhöhten Preisbereitschaft führen, die die Erzielung einer angemessen Rentabilität der Geschäftsbeziehung erleichtert. Dazu ist es jedoch notwendig, dass der Kunde aufgrund regelmäßiger bankseitiger Normübererfüllungen in der Geschäftsbeziehung einen Mehrwert sieht. ${ }^{79}$ Ein weiterer positiver Einfluss kann in der erhöhten Nachfrage nach bisher ungenutzten Bankleistungen (Cross Buying-Potenzial) oder einer erhöhten Aufgeschlossenheit gegenüber diesen (Cross Selling-Potenzial) gesehen werden. Als weitere Effekte einer intensiven Bindung können exemplarisch Weiterempfehlungen, eine erhöhte Fehlertoleranz, sowie Image- und Bekanntheitssteigerungen angeführt werden. ${ }^{80}$

Die vorstehenden Ausführungen lassen auf einen maßgeblichen Einfluss der Kundenbindung auf den langfristigen Unternehmenserfolg schließen. ${ }^{81}$ Um diese Erkenntnis sowohl strategisch als auch operativ nutzbar zu machen, ist es zunächst jedoch erforderlich die Entstehung der Kundenbindung und deren Beeinflussbarkeit einer näheren Betrachtung zu unterziehen.

\subsection{Determinanten einer intensiven Kundenbindung}

\subsubsection{Wirkmodell der Kundenbindung im Firmenkundengeschäft}

Zur Erklärung der Einflussgrößen der Kundenbindung soll im Folgenden das Modell von SEGBERS (vgl. Abbildung 8) herangezogen werden, der in einer umfassenden interdisziplinären Analyse die Geschäftsbeziehung zwischen mittelständischen Unternehmen und ihrer Hausbank untersucht hat. ${ }^{82}$

\section{Zum Konstrukt des Vertrauens vgl. Kapitel 4.2.3}

Vgl. Elsas, Ralf(2001), S. $56 \mathrm{ff}$.

Vgl. hierzu auch Kapitel 4.2.2

Vgl. Strauß, Marc-R. (2006), S. 162 f.

${ }^{81}$ Der Zusammenhang zwischen Kundenbindung und Unternehmenserfolg ist in der Literatur unbestritten, vgl. hierzu beispielsweise Bruhn, Manfred / Homburg, Christian (2005), S. 17. Offen ist jedoch noch, ob der Zusammenhang als mathematische Funktion abbildbar ist. Es wird inzwischen vermehrt von einer branchenspezifischen, nicht-linearen Interdependenz ausgegangen, vgl. Braunstein, et al. (2006), S. 78.

82 Da die Arbeit von SEGBERS auf Sekundärmaterial beruht und einige Annahmen noch nicht hinreichend empirisch validiert sind, wird das Modell - soweit die empirische Erhebung des Verfassers in Kapitel 8 nicht zu einer Validierung beitragen kann - einer kritischen Würdigung unterzogen. 


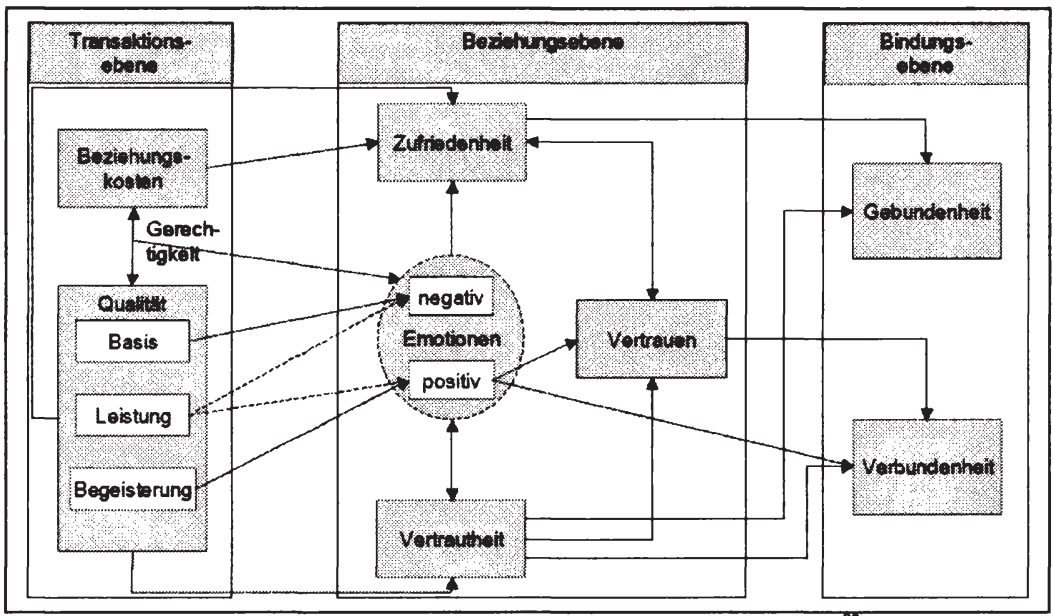

Abbildung 8: Wirkmodell einer Bankbeziehung mittelständischer Unternehmen ${ }^{8}$

Im Rahmen des Wirkmodells ergibt sich aus der Interaktion von Firmenkundenbetreuer und Unternehmensvertreter im Rahmen von Einzeltransaktionen (Transaktionsebene), eine daraus abgeleitete kumulierte Bewertung der Geschäftsbeziehung (Beziehungsebene) ${ }^{84}$ Die Erfüllung der Qualitätsanforderungen wirkt sich dabei direkt oder indirekt (über Emotionen ${ }^{85}$ ) auf die Konstrukte Zufriedenheit, Vertrautheit und Vertrauen aus. Diese haben wiederum unterschiedlichen Einfluss auf die Kundenbindung. Da die Qualitätsdimensionen, sowie die Entstehung von Zufriedenheit, Vertrautheit und Vertrauen in den nachfolgenden Kapiteln genauer erörtert werden, soll an dieser Stelle die Konzeptualisierung der Kundenbindung erläutert werden.

Diese hat sowohl eine kognitive Dimension in Form von Gebundenheit, als auch eine affektive Dimension in Form von Verbundenheit. Während die Gebundenheit als Zustand einer ökonomisch-rationalen Bindung zu interpretieren ist, basiert die Verbundenheit auf emotionalen Ursachen. ${ }^{86}$ Abbildung 9 zeigt die genannten Dimensionen in einem Schichtenmodell, um deren Bindungscharakter zu verdeutlichen.

83 Segbers, Klaus (2007), S. 339

Dieser Zusammenhang geht auf das Prozessmodell nach ALtMAN/TAYLOR zurück, vgl. Segbers, Klaus (2007), S. 45

85 Emotionen sind physiologische Erregungszustände, die die durch einen äußeren oder inneren Reiz hervorgerufen werden und als angenehm oder unangenehm empfunden werden, vgl. Kroeber-Riel, Werner / Weinberg, Peter (2003), S. 53

${ }^{86} \mathrm{Vgl}$. zu dieser Sichtweise beispielsweise Segbers, Klaus (2007), S. 335 oder Eggert, Andreas (2000), S. $121 \mathrm{f}$. . Gebundenheit ist in diesem Zusammenhang mehr als ein „NichtWechseln-Können“, es drückt vielmehr die Bindung aufgrund eines positiven Kosten-NutzenVerhältnisses aus. 


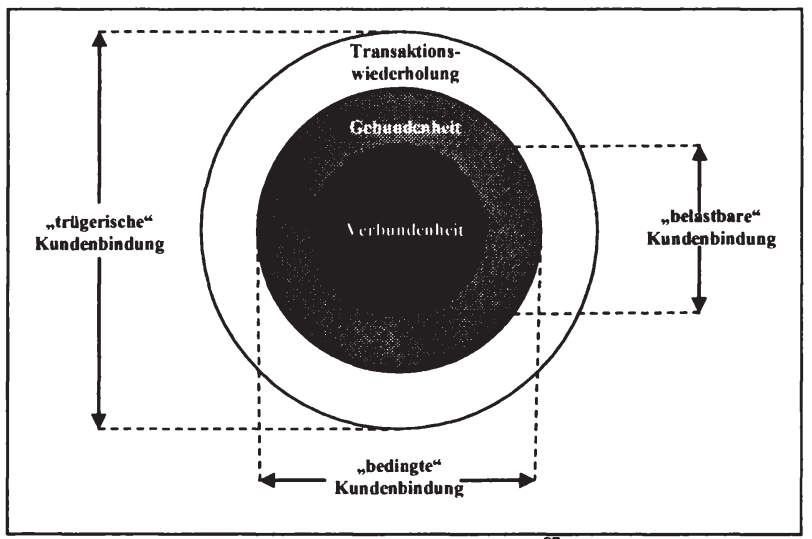

Abbildung 9: Schichten modell der Kundenbindung ${ }^{87}$

Das Schichtenmodell veranschaulicht dabei, dass eine reine Transaktionswiederholung im Sinne eines Zusatzgeschäftes nicht zwangläufig einen Bindungszustand darstellt. Es handelt sich vielmehr um eine „trügerische“ Kundenbindung, da ein erneuter Geschäftsabschluss nichts über die dahinter stehenden Motive, deren Belastbarkeit und die Wahrscheinlichkeit ihrer Aufrechterhaltung aussagt. ${ }^{88}$ So kann der Firmenkunde, der sich ein Jahr nach Eröffnung seines Geschäftskontos dazu entschließt noch eine Firmenkreditkarte zu ordern, beispielsweise aufgrund mangelnden Involvements ${ }^{89} \mathrm{zu}$ dieser Entscheidung gekommen sein.

Im Vergleich dazu kann von einem Status der Gebundenheit ausgegangen werden, wenn eine vertragliche, funktionale oder ökonomische Bindung besteht. ${ }^{90}$ Während es sich bei den beiden erstgenannten Formen der Gebundenheit um feststehende Wechselbeschränkungen handelt, stellt die ökonomische Bindung eine, auf rationalem Kalkül beruhende, Vorteilhaftigkeitsabwägung dar. Der Zustand der Gebundenheit wird in diesem Fall kundenseitig bewusst beabsichtigt. Die Stabilität der Kunde-BankBeziehung ist dabei jedoch nur so lange gewährleistet, wie die kognitive Bewertung des Kosten-Nutzen-Verhältnisses einen Ergebnisvorteil gegenüber Alternativanbietern verspricht. Insofern kann von einer „,bedingten“ Kundenbindung gesprochen werden.

87 Eigene Darstellung in Anlehnung an Stahl, Heinz K. (2006), S. 89, dort unter Verwendung anderer Begrifflichkeiten.

88 Vgl. Stahl, Heinz K. (2006), S. 89

89 Der Begriff Involvement bezeichnet den Grad des Engagements (in der Literatur häufig auch „Ich-Beteiligung“ genannt), der mit einem bestimmten Verhalten verbunden ist, vgl. hierzu Kroeber-Riel, Werner / Weinberg, Peter (2003), S. 371

9) Vgl. Georgi, Dominik (2005), S. 236. Eine vertragliche Bindung existiert im Firmenkundengeschäft z.B. bei Abschluss eines Investitionsdarlehens mit Zinsfestschreibung. Von einer funktionalen Bindung spricht man, wenn eine Leistung nur in Anspruch genommen werden kann, indem weitere Zusatzleistungen nachgefragt werden. Dies ist etwa beim Aktienkauf über eine Bank der Fall, falls dazu die Eröffnung eines kostenpflichtigen Depots erforderlich ist. 
Die stärkste Bindungswirkung kann erzielt werden, indem sich durch positive Emotionen und die Entwicklung eines besonderen Vertrauensverhältnisses ein Zustand der Verbundenheit ergibt. ${ }^{91}$ Wie sich zeigen wird ist ein solcher nur schwerlich imitierbar, wodurch im Wettbewerb eine Alleinstellung erreicht werden kann, die auf einer einzigartigen Kunde-Bank-Beziehung beruht. Um eine Differenzierung über eine solche „belastbare“ Kundenbindung zu ermöglichen, sollen nun die wesentlichen Wirkzusammenhänge erläutert werden, um die Erkenntnisse im Rahmen der weiterführenden strategischen und operativen Überlegungen zu verwenden.

\subsubsection{Kundenanforderungen im Wirkzusammenhang}

Die Verknüpfung zwischen Transaktions- und Beziehungsebene wird von SEGBERS unter Verwendung des Kano-Modells der Kundenanforderungen konzeptualisiert. ${ }^{92}$ Danach können bei der Bewertung der Transaktionsqualität drei Qualitätsdimensionen unterschieden werden, die sich unterschiedlich auf die Kundenzufriedenheit auswirken und unterschiedliche Emotionen hervorrufen (vgl. Abbildung 10).

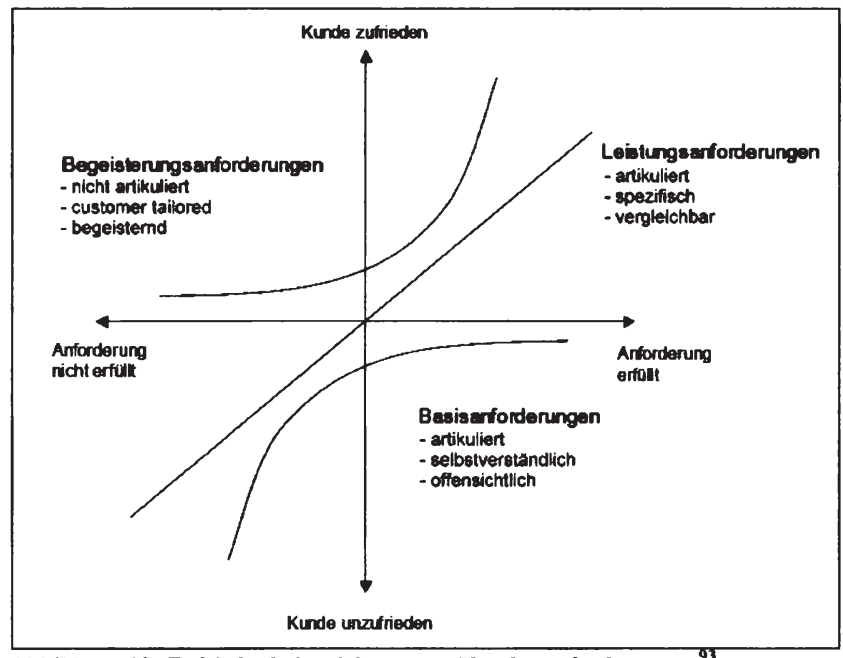

Abbildung 10: Zufriedenheitswirkung von Kundenanforderungen ${ }^{93}$

Im Folgenden sollen die Zufriedenheitswirkung und der emotive Einfluss der drei Anforderungstypen erläutert werden:

91 Vgl. Segbers, Klaus (2007), S. $337 \mathrm{f}$.

92 Vgl. Segbers, Klaus (2007), S. $312 \mathrm{ff}$.

93 Vgl. Bailom, Franz et al. (1996), S. 118, erweitert abgebildet in: Segbers, Klaus (2007), S. 325 
Die Basisanforderungen (,Dissatisfiers “) umfassen dabei sämtliche Leistungskomponenten, die kundenseitig als selbstverständlich vorausgesetzt werden. Während eine Nichterfüllung dieser Grundvoraussetzungen neben Unzufriedenheit auch negative Emotionen hervorruft, hat ihr Vorhandensein weder Zufriedenheit noch positive Emotionen zur Folge. ${ }^{94}$

Als Leistungsanforderungen („Criticals") sind solche Qualitätsmerkmale zu bezeichnen, die der Kunde einem unmittelbaren Wettbewerbsvergleich unterziehen kann. Sie werden kundenseitig explizit verlangt und führen zu einer gesteigerten Zufriedenheit bei Erfüllung, und lösen im Falle eines Fehlens Unzufriedenheit aus. ${ }^{95}$ Auch die Emotionswirkung ist je nach Grad der Erwartungserfüllung positiv oder negativ.

Begeisterungsanforderungen (,, Satisfiers") liegen vor, wenn im Rahmen einer Normübererfüllung Leistungen erbracht werden, die der Kunde nicht erwartet. Diese erscheinen ihm als wichtiger Qualitätsvorteil und lösen einen Zustand der Begeisterung aus, der durch eine überproportionale Zufriedenheitssteigerung und Emotionswirkung gekennzeichnet ist. ${ }^{\%}$

Die Gesamtzufriedenheit mit der Geschäftsbeziehung ergibt sich nun durch eine primär kognitive Aggregation der Erwartungsabgleiche auf Ebene der Einzeltransaktionen. ${ }^{97}$ Der Einfluss der unterschiedlichen Qualitätsdimensionen differiert dabei wie oben beschrieben. Zudem findet ein Abgleich zwischen Beziehungskosten und Qualität im Hinblick auf ein gerechtes Verhältnis statt. Aufgrund dieser vorrangig rational geprägten Konzeptualisierung des Zufriedenheitskonstruktes unterstellt das Wirkmodell von SEGBERS einen direkten Zusammenhang zur Dimension der Gebundenheit auf der Bindungsebene. Eine Verknüpfung mit dem Zustand der Verbundenheit erfolgt lediglich indirekt über das im nächsten Abschnitt behandelte Konstrukt des Vertrauens. ${ }^{98}$

\subsubsection{Vertrauen als zentrales Element des Wirkmodells}

In Kapitel in 4.2.1 wurde bereits ausgeführt, dass ein Zustand der Verbundenheit den stärksten Bindungscharakter aufweist. Nach SEGBERS stellt eine auf Vertrauen basierende Geschäftsbeziehung eine wesentliche Einflussgröße der Verbundenheit dar.

\footnotetext{
Vgl. z.B. Matzler, Kurt et al. (2006), S. 292 f., sowie für Beispiele Kapitel 8 und Anhang 4 Vgl. z.B. Matzler, Kurt et al. (2006), S. 292 f., sowie für Beispiele Kapitel 8 und Anhang 4 Vgl. Bailom, Franz / Matzler, Kurt (2006), S. 263, sowie für Beispiele Kapitel 8 und Anhang 4 Vgl. Grönroos, Christian (2000), S. 87.

Vgl. Segbers, Klaus (2007), S.338. Der Einfluss der Zufriedenheit auf die Verbundenheit wird in der Literatur durchaus kontrovers diskutiert. Zu einer anderen Einschätzung kommen beispielsweise TERLUTTER/WEINBERG, die postulieren, dass Verbundenheit ausschließlich auf Zufriedenheit zurückzuführen sei, vgl. Terlutter, Ralf / Weinberg, Peter (2005), S. 46.. Empirische Studien belegen jedoch, dass ein Status der Zufriedenheit nicht zwangsläufig zu einer Kundenbindung führt, was die kognitiv-basierte Interpretation von SEGBERS stützt, vgl. Neuhaus, Patricia / Stauss, Bernd (2006), S. $81 \mathrm{ff}$. .
} 
Der Vertrauensbegriff soll im weiteren Verlauf wie folgt interpretiert werden: „Vertrauen ist ein psychologischer Zustand, bestehend aus der Bereitschaft, in unsicheren Situationen mit Interdependenzen zu anderen Verletzlichkeit zu akzeptieren, basierend auf positiven Erwartungen über die Absichten oder das Verhalten anderer. ${ }^{\text {“99 }}$ Dabei ist Vertrauen als zukunftsorientiertes Phänomen zu verstehen, welches zur Komplexitätsreduktion innerhalb der Geschäftsbeziehung beiträgt. ${ }^{100}$

Um die Betrachtung der wesentlichen Verflechtungen innerhalb des Wirkmodells zu komplettieren, sollen nun die Entstehungsursachen von Vertrauen betrachtet werden. Als notwendige Bedingungen der Vertrauensbildung können die persönliche Vertrauensbereitschaft, ein gewisses Maß an Vertrautheit, sowie ein freiwilliges, persönliches und emotionales Engagement identifiziert werden.

Die obige Definition des Vertrauensbegriffes impliziert, dass die Vertrauensentstehung immer einer personellen Interaktion bedarf. Eine Voraussetzung des Vertrauensaufbaus ist dabei eine gegenseitige Vertrauensbereitschaft. ${ }^{101}$ So kann Im Rahmen einer Bankbeziehung beispielsweise kein Vertrauen entstehen, wenn der Unternehmensvertreter, etwa aufgrund von Enttäuschungen in der Vergangenheit, kein Vertrauensverhältnis wünscht. Neben der Vertrauensbereitschaft spielt die Einschätzung der Vertrauenswürdigkeit des Gegenübers - im Sinne eine „Zutrauens“ - noch eine wichtige Rolle. So ist es nur dann sinnvoll banknahe Beratungsdienstleistungen, wie z.B. die Unterstützung bei der Liquiditätsplanung, in das Leistungsportfolio zu integrieren, wenn dem Firmenkundenbetreuer die Leistungsfähigkeit kundenseitig zugesprochen wird. Zusätzlich zur hiermit angesprochenen Kompetenz stellt auch die Verlässlichkeit des Firmenkundenbetreuers eine wesentliche Komponente der Vertrauenswürdigkeit dar.

Vertrautheit kann als Grad der Bekanntheit zweier Geschäftspartner interpretiert werden, wobei zwischen persönlicher, rollenbezogener und auf der Kenntnis der Organisation beruhender Vertrautheit zu unterscheiden ist. ${ }^{102}$ Es handelt sich wie bei der Zufriedenheit um ein vergangenheitsbezogenes Konstrukt, das auf den bisherigen Erfahrungen in der Geschäftsbeziehung beruht. Vertrautheit ist als vorlaufende Variable von Vertrauen anzusehen, da es nur im Verlauf eines Bekanntheitsverhältnisses zu den interpersonellen Bewertungsprozessen kommen kann, die eine Vertrauensentwicklung zur Folge haben.

Ein persönliches, emotionales Engagement ist immer dann gegeben, wenn ein Geschäftspartner Leistungen erbringt, ,die über das übliche Maß und damit das erwartete Maß hinausgehen. "“03 Dies ist bei einer Bankbeziehung insbesondere dann

\footnotetext{
99 Burt, Ronald S. et al. (1998), S. 395, zitiert und zusammengefasst in: Segbers, Klaus (2007), S. 255

$100 \mathrm{Vgl}$. Bruhn, Manfred et al. (2006), S. 312 f.

101 Vgl. Segbers, Klaus (2007), S.287 f.

102 Vgl. Segbers, Klaus (2007), S. $272 \mathrm{ff}$., sowie die dort angegebene Literatur.

103 Vgl. Segbers, Klaus (2007), S. 289
} 
der Fall, wenn der Firmenkundenbetreuer Begeisterungsanforderungen erfüllt. ${ }^{104}$ Aus einer solchen Normübererfüllung resultieren positive Emotionen, die für eine Vertrauensbildung erforderlich sind.

Die vorstehenden Ausführungen haben gezeigt, welche Kriterien die Vertrauensentwicklung begünstigen. ${ }^{105}$ Es muss jedoch davon ausgegangen werden, dass das Zustandekommen von Vertrauen nicht vollständig logisch erklärbar ist, insofern handelt es sich um ein emergent-emotionales Phänomen. ${ }^{106}$

Zusammenfassend bleibt festzuhalten, dass die Kenntnis der Kundenanforderungen und deren Struktur, sowohl auf Ebene der Gebundenheit als auch auf Ebene der Verbundenheit, ein erhebliches Differenzierungspotenzial eröffnet und somit als Schlüsselfaktor einer erfolgreichen Kundenbindungsstrategie anzusehen ist.

\subsection{Kundenbindungsmanagement aus strategischer Perspektive}

Um die genannten Differenzierungschancen ergreifen zu können, bedarf es eines konsequenten Kundenbindungsmanagements, das eine harmonische Integration der Kundenbindung in das strategische Gesamtkonzept der Bank ermöglicht. Bevor darauf näher eingegangen wird, soll eine kurze begriffliche Einordnung des Kundenbindungsmanagements erfolgen.

\subsubsection{Begriffliche Abgrenzung des Kundenbindungsmanagements}

Eine inhaltliche Konkretisierung des Kundenbindungsmanagements ist an dieser Stelle insbesondere deshalb notwendig, da eine Reihe verwandter, teilweise synonym verwendeter Ansätze existieren.

Kundenbindungsmanagement kann als die systematische Analyse, Planung, Durchführung und Kontrolle sämtlicher auf den Kundenstamm gerichteten Aktivitäten mit dem Ziel, die Wechselbereitschaft des Kunden durch die Schaffung eines Zustands der $G e-b z w$. Verbundenheit, zu verringern und die Geschäftsbeziehung damit zu festigen $b z w . z u$ intensivieren, zusammengefasst werden. ${ }^{107}$

\footnotetext{
104 SEgBers nimmt in seiner Arbeit eine theoretische Zuordnung von Beziehungsmerkmalen zu den unterschiedlichen Qualitätsdimensionen im Kontext einer Hausbankbeziehung vor, vgl. Anhang 4. Diese wird in Kapitel 8 empirisch überprüft.

$105 \mathrm{Vgl}$. hierzu grafisch Abbildung 8

$106 \mathrm{Vgl}$. Segbers, Klaus (2007), S. $292 \mathrm{f}$.

107 In Anlehnung an Meffert, Heribert (2005), S. 149
} 
Während das Hauptaugenmerk des Kundenbindungsmanagement auf der Kundenbeziehung liegt, beschäftigt sich das Relationship Marketing auch mit anderen Anspruchsgruppen. ${ }^{108}$ Kundenbindungsmanagement kann demnach auch als Relationship Marketing im engeren Sinne verstanden werden. ${ }^{109}$

Als wichtiges Ziel des Kundenbindungsmanagement ist auch die Erhöhung der Rentabilität des Kundenportfolios zu nennen. Hier zeigt sich eine Parallele zum Retention Marketing, im Rahmen dessen allerdings nur hochrentable Kunden eine Förderung erfahren. ${ }^{110}$

Der im Zusammenhang mit dem Management einer Kundenbeziehung am häufigsten verwendete Ausdruck, dürfte der Begriff des Customer Relationship Management sein. Dieser beschäftigt sich im Kern ebenfalls mit dem Thema Kundenbindung, wird jedoch vielfach nur im informationstechnologischen Rahmen verwendet. ${ }^{11}$

Da die Positionierung der Genossenschaftsbanken die Kunde-Bank-Beziehung in den Fokus stellt und die Betrachtung über die rein informationstechnologische Perspektive hinausgehen soll, erscheint eine Verwendung des Ausdrucks Kundenbindungsmanagement hier zweckmäßig.

\subsubsection{Parameter eines integrierten Kundenbindungsmanagements}

Es wurde bereits dargelegt, dass von einem signifikanten Einfluss der Kundenbindung auf den Unternehmenserfolg auszugehen ist. Nachfolgend sollen aus den Erkenntnissen der Vorkapitel strategische Handlungsempfehlungen für das Firmenkundengeschäft erarbeitet und deren Rahmenbedingungen diskutiert werden.

Einen entscheidenden Ansatzpunkt stellen dabei die Qualitätsanforderungen der Kunden dar, da sie sowohl die Zufriedenheit beeinflussen als auch über ihre Emotionswirkung die Entstehung von Vertrauen begünstigen.

Aus den Qualitätsdimensionen lassen sich wie folgt Implikationen für die strategische Führung ableiten:

\footnotetext{
108 Vgl. Bruhn, Manfred (2001), S. 9. Relationship Marketing wird in der deutschsprachigen Literatur auch oft unter den Begriffen Beziehungsmarketing oder -management verwendet, vgl. Bruhn, Manfred / Homburg, Christian (2005), S. 8. Vgl. Bruhn, Manfred (2001), S. 10

$110 \mathrm{Vgl}$. Meffert, Heribert (2005), S. 150. Eine Rentabilitätssteigerung ist insbesondere wichtig um mit der Differenzierung auch einen ökonomischen Wettbewerbsvorteil zu erlangen, s.a. Kapitel 4.1.

111 Bruhn, Manfred / Homburg, Christian (2005), S. 7
} 
1. Erfüllung der Basisanforderungen gewährleisten,

2. Wettbewerbsfähigkeit bei den Leistungsanforderungen sicherstellen,

3. Differenzierung über Begeisterungsanforderungen erlebbar machen. ${ }^{112}$

Hierzu müssen die Kundenanforderungen allerdings bekannt sein, deshalb sollte in regelmäßigen Abständen die Anforderungsstruktur der Firmenkunden untersucht und mit dem Leistungsportfolio der Bank abgeglichen werden. Es ist zu vermuten, dass im Zeitverlauf strukturelle Verschiebungen auftreten und auch segmentspezifische Unterschiede existieren, die es zu berücksichtigen gilt. ${ }^{1{ }^{13}}$ Eine darauf folgende Anpassung des Leistungsportfolios darf ausdrücklich nicht nur auf der Ebene der klassischen Bankprodukte vollzogen werden, sondern muss sich auf die gesamte Interaktion mit dem Firmenkunden beziehen. In diesem Sinne können auch banknahe Beratungsdienstleistungen oder bestimmte Umfeldkomponenten im Rahmen des Leistungserstellungsprozesses begeisternd wirken und in das Bankangebot integriert werden. ${ }^{114}$

Für eine Bedarfsorientierung reicht es jedoch nicht aus periodisch eine allgemeine Kundenbefragung durchzuführen, vielmehr ist ein intensiver Dialog ${ }^{115}$ zwischen Firmenkundenbetreuer und Unternehmensvertreter erforderlich, um das Leistungsportfolio kundenindividuell einsetzen zu können. Zudem bedarf es einer parallelen Potenzialbetrachtung, da insbesondere die Erfüllung der Begeisterungsanforderungen sehr zeit- und kostenintensiv sein kann. ${ }^{116}$ Daher ist eine bedarfs- und potenzialorientierte Betreuungsstrategie zu entwickeln, die eine angemessene Rentabilität, einen effizienten Ressourceneinsatz und eine Begeisterungswirkung vereint.

Dazu ist ein integriertes Kundenbindungsmanagement erforderlich, das eine bestmögliche Verknüpfung und Effizienz der bindungsrelevanten Maßnahmen entlang des gesamten Vertriebsprozesses gewährleistet und somit die Differenzierung für die Firmenkunden erlebbar macht. Abbildung 11 zeigt exemplarisch die Parameter eines integrierten Kundenbindungsmanagements entlang des Vertriebsprozess im Firmenkundengeschäft:

112 Vgl. Bailom, Franz / Matzler, Kurt (2006), S. 267 bzw. speziell für das Firmenkundengeschäft Schmoll, Anton (2006), S. 63

113 Vgl. Bailom, Franz / Matzler, Kurt (2006), S. 264. Aktuelle Begeisterungseigenschaften, zu denen beispielsweise ein umfassendes Bilanzanalysegespräch gehören könnte, könnten zukünftig als selbstverständliche Serviceleistung vorausgesetzt werden.

114 Die dargestellte Homogenität der Kernleistungen lässt vermuten, dass sich Leistungs- und Begeisterungsanforderungen im Firmenkundengeschäft am ehesten durch eine herausragende Betreuungsqualität und zusätzliche Service- und Beratungsdienstleistungen erfüllen lassen, vgl. hierzu die ermittelte Anforderungsstruktur in Kapitel 8 und die daraus abgeleiteten Optimierungsansätze in Kapitel 9

115 Vgl. hierzu auch Balz, Ulrich / Bordemann, Heinz-Gerd (2004), S. 24

116 Vgl. von den Eichen, Stephan A. Friedrich et al. (2006), S. 225 f. 


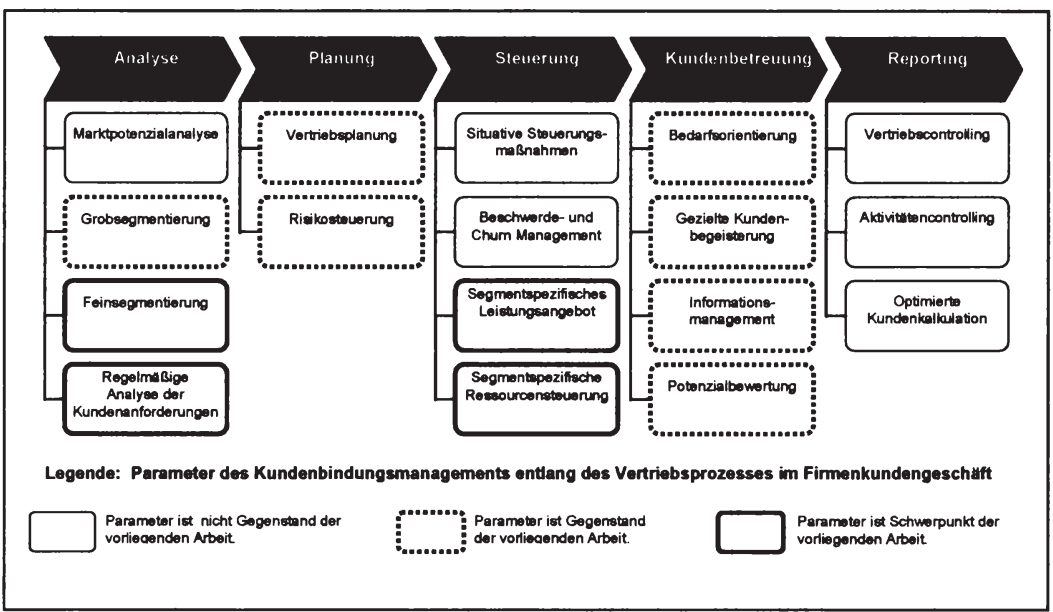

Abbildung 11: Parameter eines integrierten Kundenbindungsmanagements ${ }^{117}$

Im weiteren Verlauf der Arbeit werden alle gepunktet und fett umrandeten Parameter erneut aufgriffen und vertieft. Ein Schwerpunkt liegt dabei auf der potenzialorientierten Feinsegmentierung des Kundenportfolios, die - wie sich zeigen wird - als Ausgangspunkt einer potenzial- und bedarfsorientierten Betreuungsstrategie verstanden werden kann, sowie der Analyse der Anforderungsstruktur im Firmenkundengeschäft und der darauf aufsetzenden Optimierung des Leistungsangebotes.

In Kapitel 5 soll zunächst veranschaulicht werden, wie die Differenzierungsstrategie in der Kundeninteraktion operationalisiert werden kann. Im Zentrum der Betrachtung steht dabei der so genannte ganzheitliche Betreuungsansatz, der eine lösungs- und bedarfsorientierte Firmenkundenbetreuung intendiert.

117 Eigene Darstellung. 


\section{Operationalisierung der Differenzierungsstrategie durch Potenzial- und Bedarfsorientierung}

\subsection{Der ganzheitliche Betreuungsansatz}

Im Private Banking steht der Begriff der ganzheitlichen Kundenbetreuung schon seit einiger Zeit für einen kundenorientierten Beratungsansatz, der die Ziele und Wünsche des Kunden - an Stelle des Produktverkaufs - in den Mittelpunkt der Kundeninteraktion stellt. ${ }^{118}$ Auch im mittelständischen Firmenkundengeschäft hat die ganzheitliche Betreuung mittlerweile in einigen Vertriebskonzepten Einzug erhalten. ${ }^{119}$ Die Umsetzung dieser Betreuungsphilosophie ist hier allerdings komplexer als im Privatmarkt, da neben den betrieblichen auch private Kundenansprüche berücksichtigt werden müssen.

In der Praxis des Firmenkundengeschäfts ist festzustellen, dass der Kundendialog zunehmend aktiv, also auf Betreuerinitiative erfolgt. ${ }^{120}$ Zudem ist die produktbezogene durch eine themenbezogene Ansprache ersetzt worden. So wird neben Betreuungsterminen zu bestimmten Bedarfsfeldern auch vermehrt zu Bilanz- oder Ratinggesprächen eingeladen. Diese Ansätze bedürfen jedoch einer Intensivierung, um eine spürbare Differenzierung zu erreichen und über eine erlebbare Kundenorientierung eine nachhaltigen Wettbewerbsvorteil zu erzielen.

Dazu soll die ganzheitliche Betreuung zunächst systematisiert werden, um danach Grenzen und notwendige Schnittstellen aufzuzeigen.

\subsubsection{Systematik und Intention einer ganzheitlichen Firmenkundenbetreuung}

Grundidee einer ganzheitlichen Betreuung ist die Erfassung sämtlicher, relevanter Bedarfsfelder des Firmenkunden, um auf dieser Basis eine maßgeschneiderte Gesamtlösung zu entwickeln. Der $B V R$ hat die Bedarfsfelder im Konzept zum VR-Finanzplan Mittelstand ${ }^{121}$ beispielsweise - wie in Abbildung 12 dargestellt - klassifiziert.

Eine derart umfassende Betrachtung der Kundensituation verfolgt im Wesentlichen nachstehende Ziele:

- Intensivierung der Kundenbindung,

- Ermittlung und Ausschöpfung von Vertriebspotenzialen und die

- Erzielung eines proprietären Informationsvorteils ggü. dem Wettbewerb. ${ }^{122}$

118 Vgl. z.B. Holböck, Josef (2006), S. 170 oder zur Begriffsabgrenzung auch Schmoll, Anton (2006), S. 84

119 Vgl. u.a. BVR (2006e), S. 13.

${ }^{120}$ Es besteht hier allerdings noch Optimierungspotenzial, da nach SCHMOLL nur $20 \%$ aller Kundentermine auf Betreuerinitiative vereinbart werden, vgl. Schmoll Anton (2006), S. 83

121 Zum VR-Finanzplan Mittelstand vgl. auch die Ausführung in Kapitel 2.2.

122 Vgl. ähnlich BVR (2006e), S. 13 


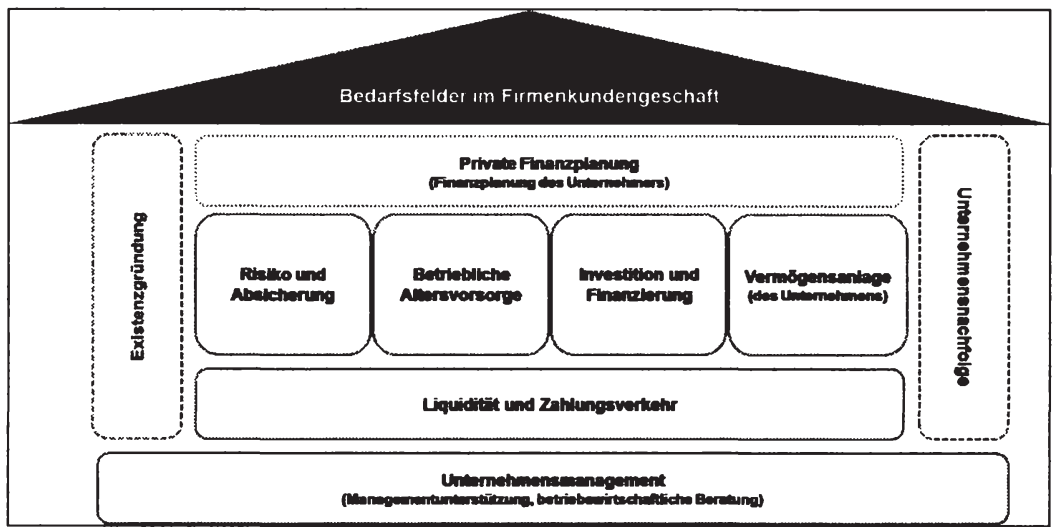

Abbildung 12: Bedarfsfelder im Firmenkundengeschăft ${ }^{123}$

In der Folge wirkt sich dies auch auf ökonomische Erfolgsgrößen wie z.B. den Kundenertrag und die Risikokosten aus. ${ }^{124}$

Der Grundansatz der Ganzheitlichkeit ist dazu sinnvoll in den Teilprozess Kundenbetreuung ${ }^{25} \mathrm{zu}$ integrieren. Aufgrund der hohen Komplexität ist dabei eine systematische Vorgehensweise erforderlich, als deren Kernelement sich ein strukturiertes Strategiegespräch anbietet. Ein idealtypischer Ablauf eines Strategiedialoges soll an dieser Stelle zur Veranschaulichung (vgl. Abbildung 13) skizziert werden: ${ }^{126}$

Nach proaktiver Terminierung eines Analysegesprächs beschäftigt sich der Firmenkundenbetreuer im Rahmen der Gesprächsvorbereitung zunächst mit der zielorientierten Aufbereitung aller kundenrelevanten Informationen. Idealerweise stehen dabei sämtliche hard und soft facts in einem CRM-System zur Verfügung. ${ }^{127}$ So können in einer elektronischen Kundeakte, beispielsweise auch eine Kontakthistorie, Beratungsergebnisse, Aktenvermerke oder Informationen zu zukünftigen Plänen des Kunden hinterlegt sein. Aus den verdichteten Kundendaten erarbeitet der Betreuer sowohl mögliche betriebliche als auch private Handlungsansätze innerhalb der kundenrelevanten Bedarfsfelder. Die heterogene Kundenstruktur muss dabei betreuerseitig

123 Vgl. BVR (2006e), S. 15

124 Vgl. Kapitel 4.1

125 Es handelt sich um einen Teilprozess des Vertriebsprozesses, vgl. Kapitel 4.3.2, Abbildung $11 .$.

126 Die Darstellung erfolgt in Anlehnung an den VR-Finanzplan Mittelstand, vgl. BVR (2006e), S. 15 ff., sowie grafisch Abbildung 13.

127 Um eine effiziente Datenanalyse und -aufbereitung zu gewährleisten, kommt dem Informationsmanagment eine besondere Wichtigkeit zu. Dazu gehört auch, dass alle wichtigen harten (z.B. Konto- und Bilanzdaten) und weichen (z.B. Aktenvermerke) Kundendaten kontinuierlich in einem CRM-Tool festgehalten werden, um sie für zukünftige Bedarfsanalysen verwenden zu können. 
berücksichtigt werden, indem er das Strategiegespräch als „Modulbaukasten“ einer kundenorientierten Betreuung versteht. ${ }^{128}$

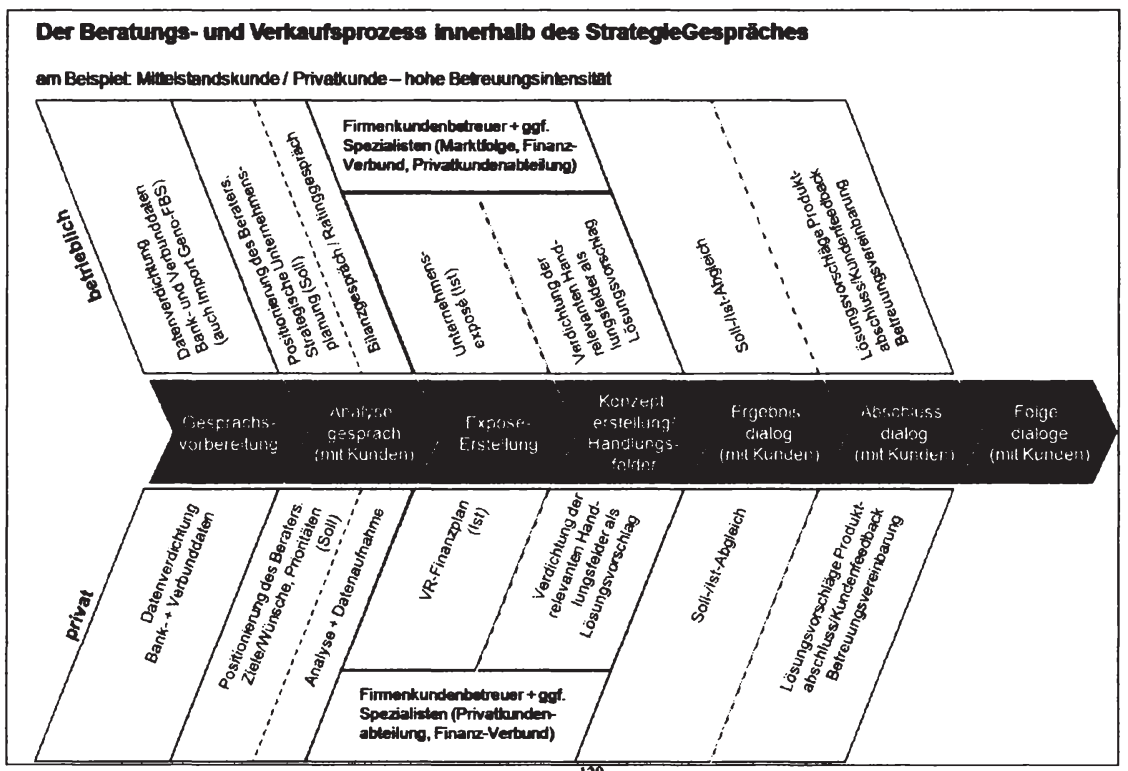

Abbildung 13: Das Strategiegespräch im Ablaufmodell ${ }^{12}$

Auf Basis des darauf folgenden strukturierten, partnerschaftlichen Analysedialoges, in welchen auch die Rating- und Bilanzanalyse integriert werden, erfolgen die Erstellung eines Unternehmensexposés und eines privaten Finanzüberblicks. Die so dokumentierte Ist-Analyse nutzt der Firmenkundenbetreuer, um unter eventueller Hinzuziehung von Fachspezialisten ein Lösungskonzept $\mathrm{zu}$ erarbeiten. In einem Ergebnisdialog werden dem Firmenkunden das Exposé und der private Finanzplan ausgehändigt. Daneben erfolgt eine Vorstellung des Lösungskonzeptes, welches in einem Abschlussgespräch verabschiedet und mit konkreten Maßnahmen für einen Einjahreszeitraum versehen wird. Auf diese Weise ergeben sich unterjährig anlassbezogene Anschlussgespräche.

Ein derart strukturierter Betreuungsprozess verspricht eine Reihe differenzierungsrelevanter Vorteile: Im Rahmen des Strategiegespräches erhält der Firmenkundenbetreuer einen umfassenden Unternehmenseinblick, den er als Informationsvorsprung im Wettbewerb nutzen kann. Hierzu bedarf es jedoch einer Softwareunterstützung, die eine

128 Vgl. hierzu auch Kapitel 5.1.2

129 BVR (2006e), S. 16 
strukturierte, auswertbare Datenaufnahme und -ablage ermöglicht. ${ }^{130}$ Im Rahmen eines konsequenten Informationsmanagements können die so gewonnenen Angaben dann entlang des gesamten Vertriebsprozesses, z.B. zur Potenzialerkennung, als Planungsgrundlage oder für Vertriebssteuerungsmaßnahmen und Folgegespräche, verwendet werden.

Des Weiteren bietet der Strategiedialog hinsichtlich mehrerer Aspekte die Möglichkeit einer potenziellen Kundenbegeisterung ${ }^{131}$ : Als erlebbare Mehrwertdienstleistung ist zunächst die dokumentierte Ist-Analyse zu nennen. Das darin integrierte Rating- und Bilanzgespräch bietet ein zusätzliches Begeisterungspotenzial, wenn die Auswertung über eine reine Ergebnispräsentation hinausgeht. So kann beispielsweise durch das Beratungstool MinD.banker eine transparente Ratinganalyse erfolgen, die auch die Simulation von Zukunftsszenarien ermöglicht. ${ }^{132} \mathrm{Die}$ in diesem Zusammenhang aufgedeckten betriebswirtschaftlichen Handlungsfelder, sind dann als Grundlage einer bankseitigen, betriebswirtschaftlichen Unterstützung des Firmenkunden verwendbar. ${ }^{133}$

Zudem findet durch die regelmäßige Kundeninteraktion ein intensiver Austausch statt, der die Entstehung von Vertrautheit begünstigt. ${ }^{134}$ Gelingt es darüber hinaus den Betreuungsprozess für den Kunden transparent zu gestalten, erleichtert dies die proaktive Terminierung der Folgegespräche. Der Firmenkunde kann die betreuerseitige Aktivität dann einem Gesamtkontext zuordnen, was tendenziell zu einer erhöhten kundenseitigen Aufgeschlossenheit führen dürfte, da sich ihm der konkrete Nutzen erschließt.

Der ganzheitliche Betreuungsansatz scheint also geeignet zu sein, um zur Operationalisierung der Kundenbindungsstrategie beizutragen. Im dargestellten Idealverlauf ist davon auszugehen, dass durch die aktive Kommunikation und die auf den Kundenbedarf zugeschnittenen Mehrwertleistungen sowohl auf kognitiver als auch affektiver Ebene eine starke Bindungswirkung erreicht wird. ${ }^{135}$

In der Realität ist die erfolgreiche Umsetzung einer ganzheitlichen Betreuungsphilosophie jedoch nicht ganz so trivial. Das Strategiegespräch ist vielmehr als elementares Instrument eines komplexen Vertriebssystems zu verstehen, welches nur dann erfolgreich ist wenn alle Rahmenparameter optimal verknüpft sind. Der bisher

130 Diese Funktionalität erfüllt beispielsweise die Software MinD.banker, die den gesamten Strategiedialog technisch umsetzt, vgl. BMS (2006), S. 3 f..

131 Zur Ermittlung der Begeisterungsanforderungen, vgl. Kapitel 8

$132 \mathrm{Vgl}$. Anhang 5. Eine strukturierte Analyse der bilanziellen Verhältnisse wird ebenfalls über die Software MinD.banker abgedeckt.

133 Die betriebswirtschaftliche Beratung stellt nach Auffassung mehrerer Autoren eine wichtige Komplementärdienstleistung im mittelständischen Firmenkundengeschäft dar und sollte zukünftig eine Intensivierung erfahren, vgl. z.B. Schmoll, Anton (2006), S. 89 oder Schmidt, Thomas (2001), S. $151 \mathrm{ff}$.

134 Vgl. Kapitel 4.2.3

135 Vgl. Kapitel 4.2.1 
eher mäßige Implementierungserfolg der Banken ist maßgeblich durch die nachfolgenden Problemfelder bedingt. ${ }^{136}$

\subsubsection{Grenzen der Ganzheitlichkeit}

Bei genauerer Betrachtung weist der ganzheitliche Betreuungsansatz auch einige Defizite auf:

Der skizzierte idealtypische Verlauf des Strategiegesprächs beginnt mit einer umfangreichen Analysephase in der sämtliche Bedarfsfelder des Firmenkunden berücksichtigt werden. Diese bedarf jedoch einer ausgeprägten Offenheit des Kunden, wie sie nur in einer vertrauensbasierten Geschäftsbeziehung vorkommt. ${ }^{137}$ Demnach ist von einer Wechselwirkung zwischen der ganzheitlichen Betreuung und dem Vertrauenskonstrukt auszugehen. Während - wie bereits beschrieben - über die aktiv gestaltete Geschäftsbeziehung und die gezielte Kundenbegeisterung die Vertrauensentwicklung gefördert wird, ist ein gewisses Maß an Vertrautheit und Vertrauen Grundlage einer umfassenden Strategieberatung. Dies muss in der Betreuungsstrategie berücksichtigt werden, indem der Betreuungsansatz modular auf den Status der Geschäftsbeziehung zugeschnitten wird. Bei skeptischen Firmenkunden sollte beispielsweise ein weniger detaillierter Analysebogen verwendet werden. Die betreuerseitige Ausarbeitung wird daraufhin logischerweise auch weniger umfassend sein. Es gilt dann den Firmenkunden über eine Nutzenargumentation von den Vorteilen einer offeneren Kommunikation zu überzeugen und ihn über Einzelkomponenten der ganzheitlichen Betreuung zu begeistern.

Das Streben nach Kundenbegeisterung birgt jedoch auch die Gefahr des Overservicing. ${ }^{138}$ Wenn die Bemühungen um Kundenbegeisterung zu ehrgeizig sind, kann dies sogar einen Wertverlust zur Folge haben. ${ }^{139}$ Bei allen Anstrengungen um die Gunst des Kunden darf also die Profitabilität der Geschäftsbeziehung nicht außer Acht gelassen werden. Deshalb ist es notwendig in der Vertriebsplanung eine ertrags- und potenzialorientierte Betreuungsintensität festzulegen. Die Kosten der ganzheitlichen Betreuung müssen dabei in die Kundenkalkulation einfließen, um Fehlentwicklungen frühzeitig zu erkennen und zyklische Anpassungen vornehmen zu können.

136 Vgl. Wildner, Georg (2006), S. 110

137 Es handelt sich dabei, um eine über das gewöhnliche Maß hinausgehende Weitergabe sensibler Informationen, vgl. hierzu die Vertrauensdefinition in Kapitel 4.2.3. SEGBERS sieht eine wesentliche Wirkung von Vertrauen in der Bereitschaft zum Austausch vertraulicher Informationen, vgl. Segbers, Klaus (2007), S. $322 \mathrm{f}$..

138 Vgl. Matzler, Kurt / Stahl, Heinz K. (2000), S. 123

139 Vgl. von den Eichen, Stephan A. Friedrich et al. (2006), S. 226 
Insbesondere bei Regionalbanken bestehen hier jedoch vielfach noch erhebliche Kalkulationsmängel. ${ }^{140}$ Des Weiteren fehlt eine systematische Erhebung des Kundenpotenzials oft vollständig. ${ }^{141}$

Ein weiteres Problem des ganzheitlichen Ansatzes stellt der Engpassfaktor Zeit dar. Die Vielzahl der Betreuungsengagements und die hohe Belastung durch nicht betreuungsbezogene Tätigkeiten bedürfen eines konsequenten Ressourcenmanagements und einer Priorisierung der Betreuungsaktivitäten, um eine erfolgreiche Marktbearbeitung zu ermöglichen. ${ }^{142}$

Bevor das Ressourcenproblem in Kapitel 5.2 vertiefend behandelt wird, soll an dieser Stelle noch das Handlungsfeld Personalentwicklung angeführt werden. So muss die ganzheitliche Betreuungsphilosophie in der Aus- und Weiterbildung der Mitarbeiter und im Führungsverständnis Einzug erhalten. Ziel der Vertriebsführungskräfte muss es vor allem sein, eine vollständige Akzeptanz der kundenorientierten Betreuung zu gewährleisten. ${ }^{143}$

\subsection{Die Problematik einer effizienten Ressourcenallokation}

Eine effiziente Ressourcensteuerung stellt eine wesentliche Erfolgsdeterminante eines ganzheitlichen Vertriebssystems dar. Das Bestreben des Vertriebsmanagements muss es sein, die knappen Betreuungsressourcen nutzenoptimal der Bedarfs-, Ertrags- und Potenzialsituation der Firmenkunden zuzuordnen.

Um hier Optimierungspotenzial zu erkennen erscheint es zunächst notwendig die Gesamtressourcen eines Firmenkundenbetreuers zu ermitteln und sich die Kapazitätsverwendung anzusehen. ${ }^{144}$ Danach werden in den Kapiteln 5.2.2 und 5.2.3 zwei Ansätze zur Effizienzsteigerung diskutiert.

\subsubsection{Zeitprofil eines Firmenkundenbetreuers}

Zur Bestimmung der Gesamtkapazität eines Firmenkundenbetreuers ist dessen betriebliche Anwesenheitszeit zu errechnen:

140 Beispielsweise bietet das in den meisten Genossenschaftsbanken eingesetzte Controllingsystem $V R$-Control derzeit noch nicht vollständig die Möglichkeit Verbunderträge in der Kundenkalkulation zu berücksichtigen, vgl. zur Problematik im Privatkundengeschäft auch Färber, Bernd / Hopfner, Wilfried (2006), S. 41

141 Zur Potenzialermittlung unter Ausnutzung von Wechselbeziehungen entlang des Vertriebsprozesses vgl. Kapitel 7.

143 Vgl. BVR (2006e), S. 30

144 Vgl. Kapitel 5.2.1 


\begin{tabular}{|c|c|}
\hline 230,0 Tage & Jahresarbeitstage \\
\hline .10 Tage & Seminare \\
\hline . 5 Tage & Krankheit \\
\hline 215 Tage & Nettoanwesenheit \\
\hline x $8 \mathrm{~h}$ & Arbeitsstunden pro Tag \\
\hline $1.720 \mathrm{~h}$ & Nettoanwesenheit \\
\hline
\end{tabular}

Tabelle 2: Anwesenheitszeit eines Firmenkundenbetreuers ${ }^{14}$

Hinsichtlich der Ressourcenverwendung kann grundsätzlich zwischen Tätigkeiten im Rahmen des Teilprozesses Kundenbetreuung und sonstigen bankinternen Aktivitäten unterschieden werden. SCHMOLL hat in diesem Zusammenhang einige hundert Firmenkundenbetreuer zu ihrem Ressourceneinsatz befragt. Abbildung 14 zeigt die Verteilung der Aufgaben auf die beiden Oberkategorien:

\begin{tabular}{|c|c|}
\hline $\begin{array}{c}\text { Kundenbetreuung I } \\
\text { marktgerichtete Aktivitäten }\end{array}$ & Bankinterne Aktivitäten \\
\hline $\begin{array}{l}\text { - Beratungs- und Verkaufsgespräche } \\
\text { - Ratinggespräche, sonstige Spezial-Beratung } \\
\text { - Betriebsbesichtigungen } \\
\text { - Kundentelefonate } \\
\text { - Gesprächsvorbereitung (Pre Sales) } \\
\text { - Angebotserstellung } \\
\text { - Akquisition (Neukundengewinnung) } \\
\text { - Kundenveranstaltungen } \\
\text { - Öffentlichkeitsarbeit / Repräsentation }\end{array}$ & $\begin{array}{l}\text { - Gesprächsnachbereitung } \\
\text { - Kreditantrag / Kreditprotokoll } \\
\text { (Vorbereitung Kreditbeschluss) } \\
\text { - Ratingerstellung } \\
\text { - Wertermittlungen } \\
\text { - Listenbearbeitungen } \\
\text { (ÜZ-Listen usw.) } \\
\text { - Disposition } \\
\text { - Unterlagenbeschaffung } \\
\text { - Schriftverkehr / Diverse Berichte } \\
\text { - Interne Besprechungen / } \\
\text { Projektgruppen } \\
\text { - Weiterbildung }\end{array}$ \\
\hline
\end{tabular}

Abbildung 14: Aufgaben des Firmenkundenbetreuers nach Vertriebsorientierung ${ }^{1+6}$

Obwohl nach der gewählten Zuordnung die markt- bzw. kundengerichteten Aufgaben einer eher weiten Definition folgen, verwenden Firmenkundenbetreuer nach dem Ergebnis der Untersuchung durchschnittlich nur 25 bis 30 Prozent ihrer Anwesenheitszeit für die Kundeninteraktion. In diesem Zusammenhang sind die Begriffe Nettovertriebszeit und Nettomarktzeit voneinander abzugrenzen. Während der Erstere, die für vertriebsgerichtete Tätigkeiten zur Verfügung stehende Zeit meint ${ }^{147}$, folgt der Letztgenannte einer engeren Interpretation. Demnach bezeichnet die Nettomarktzeit die Zeit, „die der Kundenbetreuer im persönlichen oder telefonischen Beratungs- oder Akquisitionsgespräch mit Neu- und Bestandskunden verbringt.“" ${ }^{\text {"1 }}$

145 In Anlehnung an Krauß, Carsten (2006), ohne Seitenangabe

$146 \mathrm{Vgl}$. Schmoll, Anton (2006), S. 133

147 Vgl. Schmoll, Anton (2006), S. 132

148 Vgl. Käser, Burkhard et al. (2004), S. 18 
Abbildung 15 verdeutlicht die Begriffsabgrenzung noch einmal grafisch unter Einbindung des ermittelten durchschnittlichen Zeitprofils von SCHMOLL: ${ }^{149}$

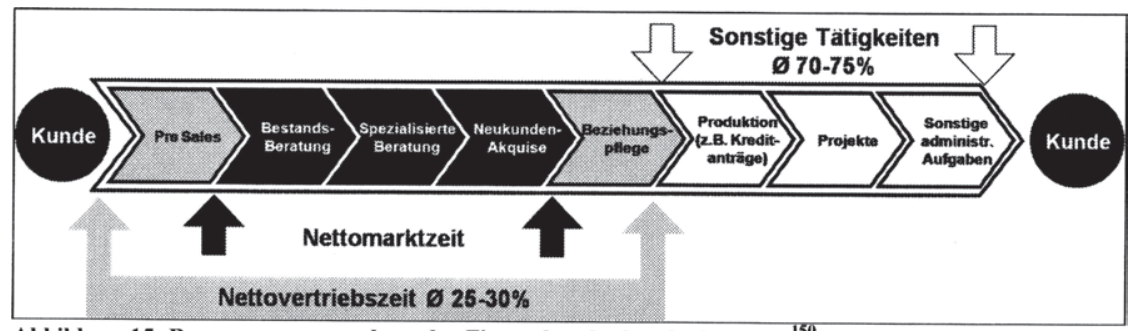

Abbildung 15: Ressourcenverwendung der Firmenkundenkundenbetreuer ${ }^{150}$

\subsubsection{Produktivitätssteigerung durch Erhöhung der Nettomarktzeit}

Unter Rentabilitätsgesichtspunkten ist es erforderlich, einen stärkeren Fokus auf wertschöpfende Tätigkeiten zu richten. Die Interdependenz zwischen Nettomarktzeit und Rentabilität konnte bereits empirisch belegt werden. Abbildung 16 zeigt das Banken mit hohen Rentabilitätswerten deutlich höhere Nettomarktzeiten aufweisen, als ihre Wettbewerber:

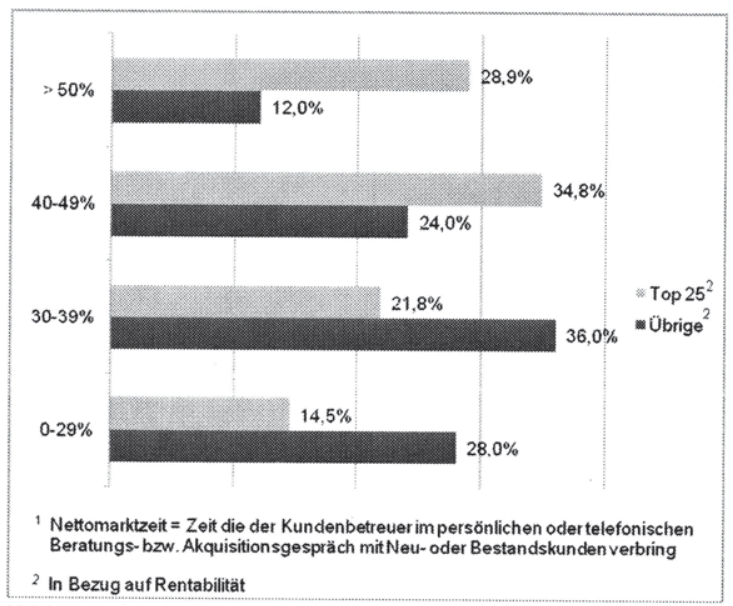

Abbildung 16: Nettomarktzeiten der Firmenkundenbetreuer nach Bankrentabilität ${ }^{151}$

149 Schmoll weist bezüglich der errechneten Zeitprofile auf große Schwankungsbreiten je nach Bankgröße, Ablauf- und Aufbauorganisation, Kundenstruktur und IT-Infrastruktur hin, vgl. Schmoll, Anton (2006), S. $133 \mathrm{f}$.

${ }^{150}$ Eigene Darstellung, Bezeichnungen und Werte in Anlehnung an Schmoll, Anton (2006), S. 133

151 Vgl. Käser, Burkhard et al. (2004), S. 18 
Einen Optimierungsansatz zur Intensivierung der ganzheitlichen Firmenkundenbetreuung stellt nun die Entlastung des Betreuers hinsichtlich nicht vertriebsbezogener Aktivitäten dar. Diese kann erreicht werden, indem administrative Tätigkeiten auf andere Aufgabenträger verlagert werden. Eine signifikante Produktivitätssteigerung ist dabei nur durch eine Veränderung der Ablauf- und Aufbauorganisation möglich. So kann beispielsweise durch den Einsatz bzw. die stärkere Integration einer Vertriebsassistenz eine Steigerung der Nettomarktzeit realisiert werden.

Die personenspezifische Aufgabenverteilung entlang des Vertriebsprozess soll an dieser Stelle allerdings nicht weiter vertieft werden. Im Folgenden steht vielmehr die optimale Verzahnung sämtlicher Teil- und Subprozesse im Vordergrund, um diese im Rahmen eines potenzialorientierten Kundenportfoliomanagements für eine effiziente Ressourcensteuerung zu nutzen.

\subsubsection{Gezielte Steuerung der Vertriebsressourcen durch Kundenportfolio- management}

Neben der Steigerung der Nettomarktzeit kommt der nutzenoptimalen Verwendung der zur Verfügung stehenden Vertriebsressourcen eine besondere Wichtigkeit zu. Diese setzt jedoch eine genaue Kenntnis der Bedarfssituation der Firmenkunden voraus, um auf dieser Basis die Betreuerkapazitäten gezielt entlang des Kundenportfolios $\mathrm{zu}$ verteilen. ${ }^{152}$ Die dafür notwendige Transparenz wird durch einen ganzheitlichen Betreuungsansatz entscheidend begünstigt. ${ }^{153}$ Allerdings muss die Betreuungsintensität innerhalb der ganzheitlichen Beratung - wie bereits angeführt - einer Kosten-NutzenAbwägung unterliegen. Somit besteht hier eine Interdependenz, die in den weiteren Überlegungen zu beachten ist.

Für das Firmenkundengeschäft der Genossenschaftsbanken bietet es sich an die Kundensegmentierung zu erweitern und im Rahmen des Kundenbindungsmanagements so zu integrieren, dass ein der Kundensituation und den Bankanforderungen angemessener Ressourceneinsatz gewährleistet wird. Nach Diskussion einiger methodischer Segmentierungsansätze in Kapitel 6, soll in Kapitel 7 ein Modell zur potenzialorientierten Kundensegmentierung entwickelt werden, dass sich schlüssig in den Gesamtkontext eines integrierten Kundenbindungsmanagements einfügt.

152 Vgl. Käser, Burkhard et al. (2004), S. 10

153 Vgl. Kapitel 5.1.1 


\section{Methodische Instrumentarien zur Segmentierung eines Kundenportfolios}

\subsection{Grundlagen der Kundensegmentierung}

Unter einer Kundensegmentierung kann allgemein die Aufteilung eines Kundenportfolios in intern homogene und extern heterogene Untergruppen verstanden werden. ${ }^{154}$ In der vorliegenden Arbeit soll jedoch eine differenziertere Betrachtung erfolgen, indem zwischen einer strategischen Grob- und einer potenzialorientierten Feinsegmentierung unterschieden wird.

Während die Grobsegmentierung auf Geschäftsfeldebene erfolgt, findet die Feinsegmentierung auf Betreuerebene statt und bildet die Grundlage einer differenzierten Marktbearbeitung innerhalb des betreuerspezifischen Kundenportfolios. ${ }^{155}$ Die merkmalsbezogene Kundenklassifizierung ist im Firmenkundengeschäft deutscher Individualbanken weit verbreitet. ${ }^{156}$ Daher sollen nachfolgend schwerpunktmäßig methodische Ansätze behandelt werden, die eine Feinsegmentierung ermöglichen. Trotzdem erscheint es sinnvoll, zunächst überblicksartig die wesentlichen Verfahren zur Grobklassifizierung vorzustellen, um im weiteren Verlauf Unterschiede herauszustellen und den Komplementärnutzen eines zweistufigen Segmentierungssystems zu verdeutlichen.

\subsection{Strategische Grobsegmentierung}

Bei den Verfahren der Kundensegmentierung lassen sich grundsätzlich ein- und multidimensionale Ansätze unterscheiden.

Zur strategischen Segmentierung auf Ebene des Geschäftsfeldes Firmenkunden werden zumeist eindimensionale Konzepte verwendet, die eine Segmentunterscheidung anhand eines bestimmten Merkmals vornehmen. Ein klassisches Kriterium stellt hier das Aktivvolumen dar, dessen Eignung aber äußerst fragwürdig ist, da es keine Rückschlüsse auf die ganzheitliche Bedarfssituation des Kunden zulässt und mitunter starken Schwankungen unterliegt. ${ }^{157}$ In einer empirischen Produktnutzungsanalyse im Firmenkundengeschäft konnte der Umsatz als wesentlicher Treiber des Bedarfs an Bankdienstleistungen identifiziert werden. ${ }^{158}$ Daher scheint dieser als Segmentierungsmerkmal eher geeignet zu sein. Daneben ist als weiteres häufig verwendetes Kriterium die Branchenzugehörigkeit zu nennen. Eine branchenorientierte Grobsegmentierung ist in Genossenschaftsbanken aufgrund von Regionalität und

\footnotetext{
154 Vgl. Bruhn, Manfred / Meffert, Heribert (2006), S. 140

$155 \mathrm{Vgl}$. Käser, Burkhard et al. (2004), S. 12

156 Vgl. Bufka, Jürgen / Eichelmann, Thomas (2002), S. 125

157 Vgl. Käser, Burkhard et al. (2004), S. 10

158 Vgl. Schmoll, Anton (2006), S. 68
} 
Unternehmensgröße nur begrenzt umsetzbar, da es dafür i.d.R. an der notwendigen Kundentiefe fehlt. ${ }^{159}$

Als Beispiel für einen multidimensionalen Ansatz im Firmenkundengeschäft ist das Konzept der Unternehmensberatung Roland Berger anzuführen, dass eine direkte Ermittlung bedarfshomogener Segmente anhand der Leistungsanforderungen der Firmenkunden vorsieht. ${ }^{160}$ Hierbei werden, die auf Basis einer Kundenbefragung ermittelten Anforderungen. mittels multivariater Analysemethoden zu homogenen Clustern zusammengefasst. ${ }^{161}$ Im Falle des Regionalinstitutes für welches das Konzept entwickelt wurde ergab die Clusteranalyse fünf Kundensegmente. Im Rahmen der Unternehmensbefragung konnte durch zusätzliche Erhebung der Kundenzufriedenheit ein empirischer Nachweis der Vorteilhaftigkeit eines bedarfsorientierten Ansatzes ggü. ausschließlich merkmalsorientierten Verfahren nachgewiesen werden. Hierzu wurden - jeweils für die Größen-, Branchen- und Bedarfsklassifizierung - Abweichungen von den mittleren Bedarfsstrukturen errechnet und mit dem Gesamtzufriedenheitsmaß regressiert. ${ }^{162}$

Der Segmentierungsvorschlag von Roland Berger erfolgt ausschließlich anhand psychographischer Merkmale. ${ }^{163}$ Die Sinnhaftigkeit dieser Vorgehensweise ist in Frage zu stellen, da die dadurch hervorgerufene Komplexität der Grobsegmentierung einem geringen Nutzen gegenübersteht. Zwar wurde die Wichtigkeit der Bedarfsorientierung schon in den Ausführungen zur ganzheitlichen Betreuung herausgestellt, es erscheint jedoch nicht zweckmäßig diese bereits in der Grobklassifizierung in das Segmentierungskonzept einzubinden. So unterliegt die Bedarfsstruktur im Firmenkundengeschäft einem dynamischen Wandel, der im Falle einer bedarfsorientierten Grobsegmentierung zu Wanderungsbewegungen innerhalb der Cluster führen würde. Bei einer stringenten Umsetzung des Konzeptes hätte dies auch sich verändernde Betreuungszuständigkeiten zur Folge, die in Diskrepanz zu einer kontinuierlichen, vertrauensbasierten Kundenbetreuung stünden. Zudem erfolgt eine aggregierte Betrachtung der Leistungsanforderungen im Rahmen der Cluster-Zuweisung, welche sich nur begrenzt für die Marktbearbeitung eignet. ${ }^{164}$ Ein weiterer Aspekt ist die fehlende Potenzialbetrachtung, denn das Modell führt keinen Abgleich zwischen den Produktbedarfen und ihrer Inanspruchnahme durch.

159 Dies deckt sich auch mit der strategischen Vorgabe des BVR, vgl. auch Kapitel 2.2-

16) Vgl. zur Vorgehensweise auch Anhang 6

161 Vgl. Bufka, Jürgen / Eichelmann, Thomas (2002), S. 130. Methodisch erfolgt die Segmentbildung auf Basis einer Clusteranalyse und einer nachgeschalteten Diskriminanzanalyse, vgl. zu den Verfahren u.a. Backhaus, Klaus et al. (2006), S. $489 \mathrm{ff}$.

162 Vgl. Bufka, Jürgen / Eichelmann, Thomas (2002), S. 135 f.. Zur Regressionsanalyse vgl. u.a. Backhaus, Klaus et al. (2006), S. $45 \mathrm{ff}$.

$163 \mathrm{Vgl}$. auch Übersicht in Anhang 7

164 So weist das Beispiel-Kreditinstitut in den Clustern 1-3 und 5 einen hohen Bedarf im Kreditgeschäft aus, vgl. Bedarfsprofile je Kundensegment in Anhang 6. Durch die Zusammenfassung der Anforderungen zu Bedarfsgruppen gehen betreuungsrelevante Impulse verloren. Die Aussage, dass Unternehmen in vorgenannten Clustern tendenziell einen hohen Kreditbedarf haben, ist zu pauschal um sie in Kundengesprächen zielführend zu nutzen. 
Für das genossenschaftliche Firmenkundengeschäft wird durch den $B V R$ - wie bereits in Kapitel 2.2 dargelegt - eine (zunächst) eindimensionale Grobsegmentierung nach dem Umsatzkriterium vorgeschlagen. Aufgrund der vorstehenden Überlegungen findet das multidimensionale Konzept zur Grobklassifizierung in der vorliegenden Arbeit keine Anwendung, stattdessen wird dem verbandsseitig vorgeschlagenen Verfahren gefolgt, da es eine hohe Operationalisierbarkeit und weite Praxisverbreitung aufweist. ${ }^{165}$

Die Bedarfs-, Potenzial- und Rentabilitätssituation werden in der Feinsegmentierung berücksichtigt, die auf der umsatzbezogenen Grobsegmentierung aufsetzt. Im Folgenden werden Verfahren vorgestellt, die für die Bildung von Subsegmenten konzeptionell relevant sind.

\subsection{Feinsegmentierung}

\subsubsection{ABC-Analyse}

Die $\boldsymbol{A B C}$-Analyse ist ein Verfahren, dass ein strategisches Geschäftsfeld oder eine Kundengruppe anhand der Rentabilität untergliedert. ${ }^{166}$ Insofern kann die Methode sowohl zur Grobklassifizierung als auch zur weiteren Kategorisierung der Subsegmente eingesetzt werden. ${ }^{167} \mathrm{Da}$ in dieser Arbeit eine Grobsegmentierung nach dem Umsatzkriterium vorgeschlagen wird, soll das Konzept hier auf seine Eignung zur Feinsegmentierung überprüft werden:

Bei einem Einsatz im Firmenkundengeschäft wird eine Einteilung der Kundengruppen hinsichtlich ihres Beitrags zum Geschäftserfolg und/oder zum Geschäftsvolumen vorgenommen. ${ }^{168}$ Für die Erfolgsbetrachtung wird der Kundendeckungsbeitrag herangezogen. Das Geschäftsvolumen wird beispielsweise anhand von Kriterien wie Passiv-, Aktivvolumen oder der Kontenumsatz abgebildet. Die Segmentzuordnung erfolgt in den Kategorien:

- $\mathrm{A}=$ überdurchschnittlich,

- $\mathrm{B}=$ durchschnittlich und

- $\mathrm{C}=$ unterdurchschnittlich. ${ }^{169}$

165 Die zeb/-Firmenkundenstudie kommt ebenfalls zu dem Ergebnis, dass das Umsatzkriterium ein geeignetes Merkmal zur Grobsegmentierung ist, vgl. Käser, Burkhard et al. (2004), S. $11 \mathrm{f}$. Vgl. Schröder, Gustav Adolf (2001), S. 596

Vgl. Schulz, Thomas Christian (2005), S. 83

Vgl. Schröder, Gustav Adolf (2001), S. 597

Vgl. Schröder, Gustav Adolf (2001), S. 596 
Untersuchungen bestätigen, dass als Ergebnis zumeist eine Verteilung der Kundengruppen nach dem Pareto-Prinzip entsteht. Auf das Firmenkundengeschäft übertragen bedeutet dies, dass mit $20 \%$ der Firmenkunden $\mathbf{8 0 \%}$ des Gesamtergebnisses erwirtschaftet werden. ${ }^{170}$

Auf Basis der ABC-Analyse erfolgt je nach Klassifizierung eine Individual-, Standardoder Mengenbetreuung, die sich hinsichtlich Betreuungsintensität und Spezialisierungsgrad unterscheidet. ${ }^{171}$ Aufgrund der ausschließlich ertrags- bzw. volumensbasierten Segmentierung ist das Verfahren jedoch als sehr einseitig zu bezeichnen. So wird die Geschäftsbeziehung lediglich vergangenheitsbezogen betrachtet, durch den fehlenden Zukunftsbezug können Potenzialkunden nicht identifiziert werden. ${ }^{172}$ Zusammenfassend ist also festzustellen, dass die Methode aufgrund ihrer Eindimensionalität nur die Kundenrentabilität ausreichend einbezieht. Die ABC-Klassifizierung ist damit wegen der unzureichenden Bedarfs- und Potenzialorientierung für eine effiziente Marktbearbeitung zu ungenau.

\subsubsection{Portfolio-Analyse}

Unter dem Begriff Portfolio-Analyse kann im Dienstleistungskontext die Positionierung von dienstleistungsbezogenen Analyseobjekten nach internen und externen Erfolgsfaktoren in einer zweidimensionalen Matrix verstanden werden. ${ }^{173}$

In diesem Abschnitt sollen zunächst die beiden wohl bekanntesten Portfolio-Ansätze das Marktanteils-Marktwachstums-Portfolio und das Wettbewerbsvorteils-Marktattraktivitäts-Portfolio vorgestellt werden. Es handelt sich hierbei, um strategische Ansätze, deren Übertragbarkeit auf die Feinsegmentierung eines Kundenportfolios überprüft werden soll. In diesem Zusammenhang sollen auch die für das Bankgeschäft entwickelten Portfolio-Methoden von SCHRÖDER und SCHMOLL gewürdigt werden.

Das Marktanteils-Marktwachstums-Portfolio (BCG-Matrix) wurde von der amerikanischen Unternehmensberatung Boston Consulting Group ursprünglich zur Positionierung strategischer Geschäftseinheiten (SGE) entwickelt. ${ }^{174}$ Dabei werden der relative Marktanteil der Geschäftseinheit auf der horizontalen und das Markwachstum der Zielmärkte auf der vertikalen Achse eines Portfolios dargestellt. ${ }^{175}$ Der Umsatzanteil der jeweiligen $S G E$ wird durch unterschiedlich große Kreise symbolisiert. Die Matrix gliedert sich in die vier Felder Question Marks, Stars, Cash Cows und Poor Dogs, die in Anhang 8 näher beschrieben werden. Je nach Positionierung der Geschäftseinheiten

170 Vgl. Schmoll, Anton (2006), S. 66

171 Vgl. Schröder, Gustav Adolf (2001), S. 597

172 Vgl. u.a. Köhler, Richard (2005), S. 409

173 Vgl. Bruhn, Manfred / Meffert, Heribert (2006), S. 174

174 Eine strategische Geschäftseinheit umfasst ein Geschäftsfeld, für das getrennt vom Rest des Unternehmens eine eigene Planung erstellt werden kann, vgl. auch Bliemel, Friedrich / Kotler, Philip (2005), S. 117.

175 Vgl. Bliemel, Friedrich / Kotler, Philip (2005), S. 117 f. 
existieren unterschiedliche Normstrategien, die dem Management als Handlungsleitlinien zur Verfügung stehen. ${ }^{176}$

Als erster der vorgestellten Segmentierungsansätze bildet die BCG-Methode neben der Betrachtung der aktuellen Marktposition über die Dimension Marktwachstum auch einen Zukunftsbezug ab. Die Portfoliodarstellung erscheint anschaulich, übersichtlich und aussagekräftig. Zu kritisieren ist, dass das Modell die Realität zu stark vereinfacht, indem es unterstellt, dass die Entwicklung von Geschäftseinheiten ausschließlich durch den relativen Marktanteil und das Marktwachstum determiniert wird.

Eine detailliertere Vorgehensweise sieht das mit Hilfe der Unternehmensberatung McKinsey entwickelte Wettbewerbsvorteils-Marktattraktivitäts-Portfolio des General Electric-Konzerns vor. ${ }^{177}$ Es handelt sich dabei um eine Multifaktorenmethode bei der die verschiedenen Einflussfaktoren auf die Portfolio-Dimensionen eigene Wettbewerbsstärke (x-Achse) und Marktattraktivität (y-Achse) berücksichtigt werden. ${ }^{178}$ Dies erfolgt indem die Faktoren im Rahmen eines Scoring-Modells gewichtet und bewertet werden, so dass schließlich für die betrachteten Dimensionen ein indexiertes Ergebnis errechnet wird. ${ }^{179}$ Nach Ermittlung der Indexwerte können diese in der in neun Felder untergliederten Klassifizierungsmatrix abgebildet werden. Unterschiedlich große Kreise kennzeichnen dabei die Marktgröße der SGEs, der Marktanteil wird durch eine entsprechende farbliche Abhebung der Kreise verdeutlicht. ${ }^{180}$ Je nach Positionierung werden auch hier unterschiedliche Normstrategien vorgeschlagen. ${ }^{181}$

Durch das Scoring-Verfahren können sämtliche, als wichtig erachtete Faktoren in die Analyse einbezogen werden. Jedoch weist die Auswahl, Gewichtung und Bewertung der Faktoren eine gewisse Subjektivität auf. Somit hängt der erfolgreiche Einsatz zu einem hohen Anteil von den analytischen und strategischen Fähigkeiten der Führungskräfte $a b$.

Die Multifaktorenmethode ist problemlos auf die Betrachtung eines Kundenportfolios zu transferieren und ermöglicht somit eine umfassende Einschätzung der Bedarfs-, Potenzial- und Ertragssituation. ${ }^{182}$ Es ist jedoch ein Konzept erforderlich, dass eine ausreichende Validität gewährleistet, indem der Subjektivitätsgrad verringert wird.

\footnotetext{
176 Vgl. Bliemel, Friedrich / Kotler, Philip (2005), S. 119 f.

177 Vgl. Bruhn, Manfred / Meffert, Heribert (2006), S. 174

$178 \mathrm{Vgl}$. Schröder, Gustav Adolf (2001), S. 600

$179 \mathrm{Vgl}$. Tabelle 12 in Anhang 9

$180 \mathrm{Vgl}$. Abbildung 41 in Anhang 9

181 Vgl. Abbildung 42 in Anhang 9

182 Zur Übertragung der Multifaktorenmethode auf ein Kundenportfolio, vgl. Köhler, Richard (2005). S. $416 \mathrm{f}$.
} 
Eine erste Übertragung der McKinsey-Methode auf das Bankgeschäft erfolgte durch SCHRÖDER. Er ersetzt hierzu die Dimension Markt- durch die Kundenattraktivität, welche anhand des Geschäftsvolumens ermittelt wird. ${ }^{183}$ Das externe Kundenpotenzial stellt aus seiner Sicht die Grundlage der Dimension Eigene Wettbewerbsstärke dar. ${ }^{184}$ Das Potenzial errechnet sich als Anteil des Geschäftsvolumens am statistischen Gesamtvermögen des Kunden. Auf Basis der neun Klassifizierungsstufen werden segmentspezifische Betreuungsstrategien abgeleitet. ${ }^{185}$

SCHRÖDER nutzt jedoch die Möglichkeiten des Verfahrens nur begrenzt, indem er auf das Scoring-System komplett verzichtet. Eine Erklärung hierfür könnte darin liegen, dass der Segmentierungsansatz für das Privatkundengeschäft entwickelt wurde. Durch diese Vorgehensweise wird eine einfache Operationalisierbarkeit des Verfahrens gewährleistet. Für das Firmenkundengeschäft erscheint der Ansatz trotz seiner hohen Praktikabilität für eine Feinsegmentierung ungeeignet, da gerade im betreuungsintensiven Individualkundengeschäft eine differenzierte Erfassung der Kundensituation erforderlich ist, die über eine Klassifizierung anhand statistischer Durchschnittswerte hinausgehen sollte.

Die notwendige Detailbetrachtung im Firmenkundengeschäft wird in den Überlegungen von SCHMOLL aufgegriffen, der einige Empfehlungen zur Feinsegmentierung erarbeitet hat. Die vorgeschlagene Verfahrensweise stellt dabei einen Mix aus den bereits vorgestellten Portfolio-Methoden dar. Als Bewertungsdimensionen des Modells soll das bereits ausgeschöpfte interne und das zukünftige externe Kundenpotenzial abgebildet werden. ${ }^{186}$ Die Analyse der Einflussfaktoren der beiden Komponenten soll zu einer aggregierten Bewertung innerhalb der Portfolio-Dimensionen führen. Tabelle 3 zeigt eine Übersicht möglicher Kriterien:

\begin{tabular}{|l|l|}
\hline Kriterien - Internes Kundenpotenzial & Kriterien - externes Kundenpotenzial \\
\hline - Aktivvolumen & -Zukunftschancen der Branche \\
\hline - Passivvolumen & - Markposition innerhalb der Branche \\
\hline - Kontoumsatz & - Expansionspotenzial \\
\hline - Geschäftsvolumen & - Umsatzpotenzial \\
\hline - Produktnutzung & - Exportmöglichkeiten \\
\hline - Firmenumsatz & - Innovationskraft \\
\hline & - Investitionsneigung \\
\hline
\end{tabular}

Tabelle 3: Segmentierungskriterien nach SCHMOLL ${ }^{18}$

Für die Portfolio-Einordnung schlägt SCHMOLL eine Vier-Felder-Matrix analog der BCG-Methode vor. Dabei unterscheidet er zwischen Ideal-, Potenzial-, Beobachtungsund Standardkunden. Das Portfolio sieht demnach wie folgt aus:

183 Vgl. Schröder, Gustav Adolf (2001), S. 601

184 Vgl. Schröder, Gustav Adolf (2001), S. 602

185 Vgl. Schröder, Gustav Adolf (2001), S. 603 ff., sowie Anhang 10

186 Vgl. Schmoll, Anton (2006), S. 68

187 Vgl. Schmoll, Anton (2006), S.69 f. 


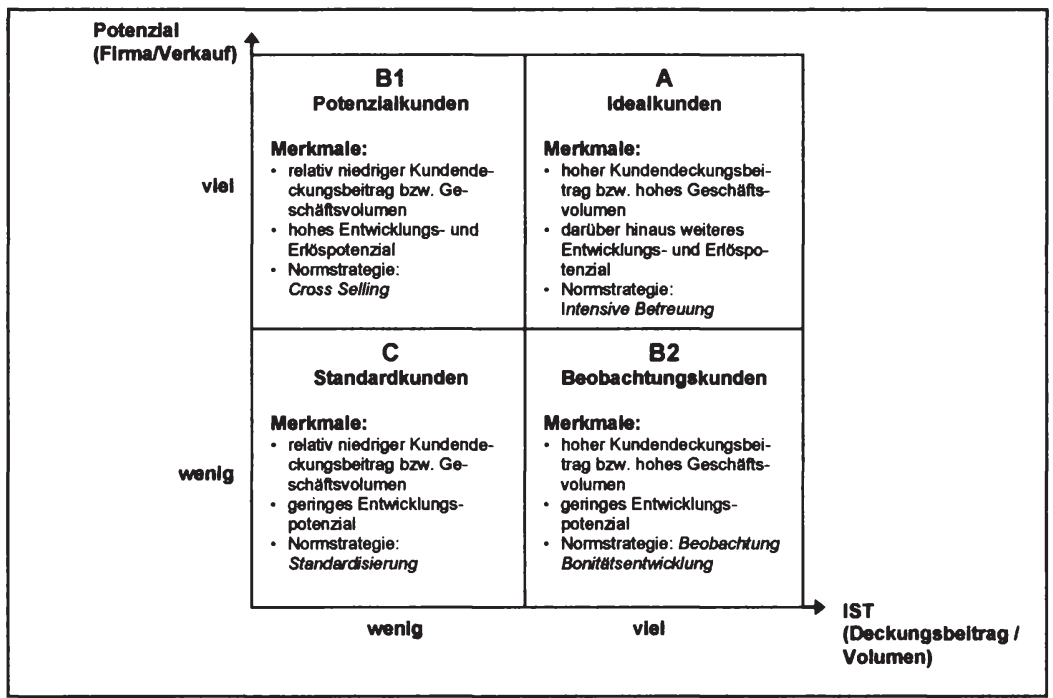

Abbildung 17: Firmenkundenportfolio nach SCHMOLL ${ }^{189}$

Durch die Mehrdimensionalität können alle aus Managementsicht erforderlichen Faktoren in den Segmentierungsansatz aufgenommen werden. Die Subjektivität wird durch die Einbindung quantitativer Segmentierungskriterien gemildert. ${ }^{189}$ Dennoch bietet auch der Ansatz von SCHMOLL keine hinreichend objektive Segmentierungslösung. Es handelt sich hier ebenfalls um ein Modell, das hinsichtlich der Kriterienauswahl, -gewichtung und -bewertung Unsicherheiten unterliegt. Trotzdem wird durch die Verfahrensweise eine Komplexitätsreduktion erreicht, die eine differenzierte Marktbearbeitung ermöglicht. ${ }^{190} \mathrm{Zu}$ kritisieren ist allerdings, dass aufgrund der Unterschiedlichkeit der vorgeschlagenen Kriterien, rückwirkend kein Soll-Ist-Abgleich des Segmentierungsergebnisses möglich ist. Zudem stellen die vorgeschlagenen Potenzialkriterien allesamt indirekte Indikatoren der zukünftigen Bedarfssituation dar.

Im Rahmen einer ganzheitlichen Betreuungsphilosophie erfolgt jedoch eine kontinuierliche Erfassung der Bedarfssituation des Kunden, so dass eine direkte Einbindung der Analyseergebnisse zweckmäßig erscheint.

188 In Anlehnung an Schmoll, Anton (2006), S. 70 f.

189 Vgl. Schmoll, Anton (2006), S.65

190 Wie bereits die anderen Methoden gezeigt haben, führt eine Segmentierung auch zu unbefriedigenden Ergebnissen, wenn sie ausschließlich auf messbaren Kriterien basiert. Die Aufnahme weicher Faktoren bietet den Vorteil, in strukturierter Form eine Einschätzung wichtiger, nicht messbarer Aspekte zu erhalten und diese im Vertriebsmanagement zu berücksichtigen. 
Im nachfolgenden Kapitel soll nun ein erweiterter Segmentierungsansatz konzipiert werden, der die von SCHMOLL vorgeschlagene Systematik grundsätzlich aufgreift, diese aber so modifiziert und weiterentwickelt, dass sie sich harmonisch Vertriebsprozess von Genossenschaftsbanken integrieren lässt. 


\section{Potenzialorientierte Kundensegmentierung}

\subsection{Anforderungsanalyse und Zieldefinition}

Die Ausführungen zum ganzheitlichen Betreuungsansatz haben bereits verdeutlicht, dass dieser nur dann erfolgreich umzusetzen ist, wenn die Marktbearbeitung hinreichend systematisiert ist. ${ }^{191}$ Dazu ist es erforderlich den Vertrieb so zu steuern, dass einerseits den Kundenbedürfnissen in ausreichender Form Rechnung getragen wird, die Betreuungsintensität andererseits aber auch an den Potenzial- und Ergebnisbeitrag des Kunden angepasst wird. Um dies leisten zu können muss eine Feinsegmentierung im Wesentlichen die folgenden Anforderungen erfüllen:

Der zu entwickelnde Ansatz soll zunächst an das Grobsegmentierungskonzept der genossenschaftlichen Bankengruppe anknüpfen und die Bedarfs-, Potenzial und Ergebnissituation je Betreuungseinheit transparent machen. Die Kundensegmentierung soll dabei engagementbezogen erfolgen und nach Möglichkeit auch den Unternehmer als Privatperson mit einbeziehen. ${ }^{192}$

Basierend auf einer genauen Kenntnis seines Kundenstamms soll der Firmenkundenbetreuer über die Feinklassifizierung in die Lage versetzt werden, die Betreuungsressourcen so einzusetzen, dass bei möglichst vielen Unternehmen eine Begeisterungswirkung eintritt und gleichzeitig ein Overservicing vermieden wird. Dabei gilt es bestehende Interdependenzen entlang des Vertriebsprozesses in der Segmentierungskonzeption zu berücksichtigen, um die verfügbaren Informationen nutzenoptimal zu verwerten. ${ }^{193}$

Auf Grundlage der Feinsegmentierung soll eine segmentspezifische Betreuungsstrategie je Subsegment entwickelt werden, die sowohl als Leitlinie für die Ressourcensteuerung als auch zur Festlegung des Betreuungsangebotes herangezogen werden kann. ${ }^{194}$ Die strukturierte Vorgehensweise soll die Produktivität des Betreuungsprozesses durch eine gezielte Potenzialausschöpfung erhöhen.

Hinsichtlich der Segmentierungskriterien hat eine differenzierte Auswahl zu erfolgen, die alle wesentlichen Einflussgrößen der Portfolio-Dimensionen berücksichtigt. Die Kriterien sollen dabei so gewählt werden, dass ein nachgelagerter Validitätsabgleich erfolgen kann. Der Subjektivitätsgrad ist dabei durch eine Mischung qualitativer und quantitativer Kriterien abzuschwächen.

\footnotetext{
191 Vgl. Kapitel 5.1.2

192 Ein wesentliche Zukunftschance wird in einer das betriebliche Engagement übergreifende Betreuung gesehen, vgl. auch SWOT-Analyse Kapitel 3.1.4.

193 Es gilt dazu ein effizientes Informationsmanagement zu betreiben, um alle segmentierungsrelevanten Informationen in die Feinklassifizierung einfließen zu lassen, vgl. Kapitel 5.1.1.

194 Vgl. hierzu Kapitel 9
} 
Die Segmentierung muss zudem eine hohe Operationalisierbarkeit aufweisen, indem sie passgenau in die Betreuungskonzeption integriert wird, um so als Ausgangspunkt des Kundenbindungsmanagements fungieren zu können. In diesem Zusammenhang gilt es auch den Segmentierungsprozess für den Betreuer praktikabel zu gestalten.

Die Subsegmente sind so zu wählen, dass hinreichend große Kundengruppen für eine differenzierte Betreuungsstrategie ermittelt werden können. ${ }^{195}$ Außerdem soll die Klassifizierung eine gewisse Zeitstabilität aufweisen.

Die Anforderungen an das Segmentierungskonzept können in nachfolgender Zieldefinition zusammengefasst werden:

Ziel des zu entwickelnden Ansatzes zur Feinsegmentierung ist die Bildung homogener Subsegmente, die eine effiziente bedarfsorientierte Betreuung ermöglichen, um eine nutzenoptimale Umsetzung der Kundenbindungsstrategie zu gewährleisten und somit eine bestmögliche Ertrags- und Potenzialausschöpfung zu erreichen. ${ }^{196}$

\subsection{Entwicklung eines Portfolio-Modells im Firmenkundengeschäft}

\subsubsection{Konzept und Dimensionierung}

Zur Erfüllung des Anforderungsprofils ist eine detaillierte Bewertung der Kundenengagements erforderlich, in die eine Reihe von Faktoren einfließen muss, damit eine adäquate Nutzung der Ergebnisse im Rahmen einer differenzierte Betreuungsstrategie erfolgen kann. Zeitlich wird der Fokus sowohl auf die gegenwärtige als auch auf die zukünftige Engagemententwicklung gelegt.

Die vorgenannte Zielsetzung soll mittels multifaktorieller Portfolio-Analyse erreicht werden, da die Multifaktorenmethode die Erfassung, Gewichtung und Bewertung aller wesentlichen Merkmale der Geschäftsbeziehung ermöglicht. Des Weiteren eignet sich der Portfolio-Ansatz sehr gut um unterschiedliche Zeitdimensionen abzubilden. Die Vorteile der vorgestellten Segmentierungsansätze sind dabei einzubinden bzw. weiterzuentwickeln.

Es gilt dazu zunächst die relevanten Faktoren festzulegen und so in zwei Dimensionen zu bündeln, dass sie in einer Portfolio-Matrix abgebildet werden können.

195 Vgl. Schmoll, Anton (2006), S. 64

196 Die potenzialorientierte Kundensegmentierung hat Einfluss auf sämtliche Instrumente des Kundenbindungsmanagements und bildet die Basis des ganzheitlichen Vertriebssystems. Sie ist wesentliche Determinante der Ressourcen- und Angebotssteuerung. 
Zur Festlegung der Portfolio-Dimensionen kann grundsätzlich der Systematik von SCHMOLL gefolgt werden, der auf der horizontalen Achse die gegenwärtige und auf der vertikalen Achse die zukünftige Kundensituation darstellt. Die Segmentierungskriterien sollen dabei unter den Dimensionsbezeichnungen Status und Potenzial subsumiert werden.

Um der strategischen Ausrichtung der Genossenschaftsbanken Rechnung zu tragen, muss die Bedarfsorientierung stärker in den Mittelpunkt der Kundensegmentierung rücken. Dazu soll sowohl die aktuelle als auch die zukünftige Bedarfssituation in der Bewertung berücksichtigt werden und somit in beide Dimensionen einfließen.

Die Problematik bei der Auswahl der Segmentierungskriterien besteht darin, dass quantitative Merkmale teilweise zu ungenau oder zu pauschalierend sind, während qualitative Kriterien der Subjektivität des Betrachters unterliegen. Daher soll der zu entwickelnde Segmentierungsansatz einen Kriterien-Mix aus hard und soft facts enthalten. Am Beispiel des Grobsegments Mittelstandskunden soll in den nächsten beiden Abschnitten die Kriterienauswahl vorgestellt und erläutert werden. ${ }^{197}$

\subsubsection{Kriterien zur Erhebung des aktuellen Status}

In die Bewertung der aktuellen Situation des Kundenengagements sollen primär Faktoren einbezogen werden, die den aktuellen Ergebnisbeitrag und die bisherige Bedarfssituation erfassen. Dabei soll - konform zum ganzheitlichen Betreuungsansatz sowohl die betriebliche als auch die private Sphäre Berücksichtigung finden. Tabelle 4 zeigt eine Übersicht der im Rahmen der Segmentierungsworkshops ausgewählten Kriterien: ${ }^{198}$

In den bankinternen Informationssystemen ist eine Vielzahl von Kundendaten gespeichert. Es gilt nun diese Informationen für die Segmentierung so zu filtern, dass der Firmenkundenbetreuer nur dann eine subjektive Einschätzung vornehmen muss, wenn die quantitativen Daten nicht aussagekräftig genug sind. Der mit der Segmentierung verbundene Zeitaufwand wird auf dieses Weise auf ein notwendiges Minimum beschränkt.

197 Die Klassifizierungskriterien müssen für jedes Grobsegment angepasst werden, um die Besonderheiten dieser großen Kundengruppen adäquat zu berücksichtigen. So darf z.B. ein regionales Handwerksunternehmen (Grobsegment „Gewerbekunde“) in der Segmentierung nicht benachteiligt werden, wenn es keine Außenhandelsdienstleistungen in Anspruch nimmt.

198

Zur Kriterienauswahl wurden unter Einbezug von Fachexperten aus der Praxis Segmentierungsworkshops im Grobsegment „Mittelstand" durchgeführt, die der Verfasser geleitet hat. Die erarbeitete Struktur ist unter geringfügigen Modifikationen auch für andere Grobsegmente einsetzbar. Diese Modifikationen sind jedoch nicht Gegenstand der vorliegenden Arbeit. 


\begin{tabular}{|c|c|}
\hline \multicolumn{2}{|l|}{ Allgemeine Kriterien } \\
\hline \multicolumn{2}{|l|}{ - Intensität der Bankverbindung } \\
\hline \multicolumn{2}{|l|}{ - Deckungsbeitrag der Betreuungseinheit p.a. } \\
\hline \multicolumn{2}{|l|}{ Deckungsbeitrag der Betreuungseinheit p.a. } \\
\hline Kriterien - Beziehung zum Unternehmen & Kriterien - Beziehung zum Unternehmer \\
\hline Leistungsanalyse - Betriebliche Bedarfsfelder & Leistungsanalyse - Private Bedarfsfelder \\
\hline \multirow{3}{*}{$\begin{array}{l}\text { Bedarfsfeld Liquidität und Zahlungsverkehr } \\
\text { Zahlungsverkehr: } \\
\text { - Kontokorrent } \\
\text { - Auslandszahlungsverkehr } \\
\text { - eBanking } \\
\text { - Kreditkartengeschäft } \\
\text { Kurzfristfinanzierung: } \\
\text { - Kontokorrent- / Termingeldkredit } \\
\text { - Factoring } \\
\text { - Avalkredit }\end{array}$} & $\begin{array}{l}\text { - Bedarfsfeld Zahlungsverkehr } \\
\text { - Kontokorrent } \\
\text { - eBanking } \\
\text { - Kreditkartengeschäft }\end{array}$ \\
\hline & $\begin{array}{l}\text { - Bedarfsfeld Immobilien und Finanzierung } \\
\text { - Dispositionskredit } \\
\text { - Private Anschaffungsdarlehen } \\
\text { - Wohnbaufinanzierungen }\end{array}$ \\
\hline & $\begin{array}{l}\text { Bedarfsfeld private Vermögensanlage } \\
\text { - Tages- und Festgeld }\end{array}$ \\
\hline \multirow{2}{*}{$\begin{array}{l}\text { Bedarfsfeld Investition und Finanzierung } \\
\text { Mittel- / Langfristfinanzierung: } \\
\text { - Bankdarlehen } \\
\text { - öffentliche Förderprogramme } \\
\text { - Leasing } \\
\text { - Vermittlung von Darlehen } \\
\text { - Währungskredite }\end{array}$} & $\begin{array}{l}\text { - Wertpapieranlage / Vermögensverwaltung } \\
\text { - Bausparen } \\
\text { - Immobilien }\end{array}$ \\
\hline & \multirow[t]{3}{*}{$\begin{array}{l}\text { Bedarfsfeld Absicherung und Vorsorge } \\
\text { - Sachversicherungen } \\
\text { - Lebensversicherungen } \\
\text { - Krankenversicherungen }\end{array}$} \\
\hline $\begin{array}{l}\text { Bedarfsfeld Vermögensanlage } \\
\text { Kurzfristanlage: } \\
\text { - Tages- und Festgeld } \\
\text { Langfristanlage: } \\
\text { - Wertpapieranlage / Vermögensverwaltung }\end{array}$ & \\
\hline $\begin{array}{l}\text { Bedarfsfeld Risiko und Absicherung } \\
\text { Absicherung: } \\
\text { - Sachversicherungen } \\
\text { Vorsorge: } \\
\text { - Betriebliche Altersvorsorge }\end{array}$ & \\
\hline \multirow{3}{*}{\multicolumn{2}{|c|}{$\begin{array}{l}\text { Branchensituation } \\
\text { - Umsatzentwicklung } \\
\text { - Kundenentwicklung im Vergleich zur Branche }\end{array}$}} \\
\hline & \\
\hline & \\
\hline $\begin{array}{l}\text { Volkswirtschaftliche Rahmenparameter - } \\
\text { Unternehmen }\end{array}$ & $\begin{array}{l}\text { Volkswirtschaftliche Rahmenparameter - } \\
\text { Unternehmer }\end{array}$ \\
\hline \multirow[t]{2}{*}{ - KfW-ifo-Mittelstandsbarometer Lagebeurteilung } & - Regionale Kaufkraftkennziffer \\
\hline & - Entwicklung der Sparquote \\
\hline
\end{tabular}

Tabelle 4: Segmentierungskriterien zur Statuserhebung

Der Status der Geschäftsbeziehung lässt sich relativ gut durch die Nutzung des vorhandenen Datenmaterials abbilden. Die benötigten Daten können aus einem 
CRM-System exportiert und somit für die Segmentierung nutzbar gemacht werden. ${ }^{199}$ Zur Statuserfassung sind daher nur wenige weiche Faktoren (in Tabelle 4 durch kursiven Schriftschnitt gekennzeichnet) abzufragen.

Insgesamt lassen sich die Kriterien den Bereichen Ergebnisbeitrag, Leistungsanalyse, Branchensituation und volkswirtschaftliche Rahmenparameter zuordnen. Da durch die Kundensegmentierung eine engagementindividuelle Betrachtung erfolgen soll, liegt der Schwerpunkt auf den beiden erstgenannten Merkmalsgruppen. Wesentliche Aspekte der Kriterienstruktur sollen nachfolgend erläutert werden:

Der Ergebnisbeitrag des Engagements wird maßgeblich am Gesamtdeckungsbeitrag der Betreuungseinheit gemessen. ${ }^{200}$ Zusätzlich wird ein Quotient aus Deckungsbeitrag und Firmenumsatz gebildet, um unterschiedliche Unternehmensgrößen in die Bewertung mit einzubeziehen. So soll es sich beispielsweise positiv auf das Segmentierungsergebnis auswirken, wenn ein relativ kleiner Mittelständler einen hohen Deckungsbeitrag aufweist.

Als weiches Merkmal ist in dieser Kriteriengruppe vom Firmenkundenbetreuer die Intensität der Bankverbindung einzuschätzen. Hier soll beurteilt werden, wie aktiv der Kunde die Geschäftsverbindung im Verhältnis zum Wettbewerb nutzt.

Im Rahmen der Leistungsanalyse wird die gegenwärtige Bedarfssituation des Kunden betrachtet. Die Bankleistungen sind zu Gruppen zusammengefasst und den zugehörigen Bedarfsfeldern zugeordnet. Es ergibt sich auf diese Weise eine bedarfsorientierte Sicht auf das Leistungsportfolio. ${ }^{201}$

Die Leistungszuordnung orientiert sich an den Vorgaben des $B V R$, der in den Konzepten zum VR-Finanzplan Mittelstand (betriebliche Sphäre) und zum VR-Finanzplan (private Sphäre) Produktübersichten je Bedarfsfeld erstellt hat. ${ }^{202}$ Diese wurden für die Segmentierung gestrafft, so dass nur solche Produkte und Dienstleistungen berücksichtigt werden, die steuerungsrelevant sind und einen Umsatzbeitrag erzielen. Dienstleistungen, die im Sinne einer Kundenbindungsstrategie zur Erzielung einer Begeisterungswirkung als kostenloser Zusatzservice angeboten werden, dürfen nicht einfließen. Es handelt sich bei selbigen um Mehrwertleistungen, die als bankseitige Investitionen in die Geschäftsbeziehung zu verstehen sind..$^{203} \mathrm{Da}$ die Volks- und Raiffeisenbanken

199 Zur Datenversorgung eignen sich Kernbankanwendungen wie z.B. bank2l (GAD eG) oder agree (FIDUCIA IT AG). Idealerweise wird die Segmentierung aber unter Nutzung firmenkundenbankspezifischer Spezialsoftware, wie MinD.banker (BMS Consulting $\mathrm{GmbH}$ ) durchgeführt, da auf diese Weise eine optimale Versorgung mit geschäftsfeldspezifischen quantitativen und qualitativen Daten gewährleistet werden kann. Es handelt sich hierbei um den periodischen Jahresdeckungsbeitrag des Engagements inklusive sämtlicher Provisionen aus dem genossenschaftlichen FinanzVerbund.

203 $\mathrm{Zu}$ Verwendung und Nutzen der Leistungsanalyse vgl. Kapitel 7.2.4.

Vgl. für die betriebliche Produktübersicht BVR (2006f).

Zum zielgerichteten Einsatz solcher Mehrwertleistungen im Rahmen der Betreuungsstrategie vgl. Kapitel 9 
derzeit i.d.R. keine bepreisten betriebswirtschaftlichen Beratungsdienstleistungen anbieten, findet das Bedarfsfeld Unternehmensmanagement in der Klassifizierung keine Berücksichtigung.

Hinter der Aufnahme der Kriteriengruppen Branchensituation und volkswirtschaftliche Rahmenparameter steckt die Überlegung, dass der Firmenkunde permanent durch das volkswirtschaftliche Umfeld tangiert wird. Auf der betrieblichen Seite kommen dazu noch branchenspezifische Einflüsse hinzu. Von diesen Prämissen ausgehend sollen die Rahmenbedingungen in das Segmentierungsergebnis einfließen, um die Kundensituation möglichst realitätsnah darzustellen.

\subsubsection{Kriterien zur Erhebung des Potenzials}

Um die Segmentierungsergebnisse einem Soll-Ist-Vergleich unterziehen zu können müssen sich die Kriterien zur Potenzialerhebung weitgehend mit Statuskriterien decken. Auf diese Weise kann rückwirkend überprüft werden, ob das gegenwärtige Statusergebnis mit der Potenzialeinschätzung des Vorjahres übereinstimmt.

Dieser Anforderung wird Folge geleistet, indem bei der Kriterienauswahl nur auf solche Kriterien zurückgegriffen wurde, die auch eine Potenzialeinschätzung ermöglichen. Die weitgehend kongruente Kriterienauflistung findet sich in Anhang 11.

Der große Unterschied zur Statusbetrachtung ist, dass die Potenzialkriterien weitestgehend der qualitativen Bewertung der Firmenkundenbetreuer unterliegen. ${ }^{204}$ An dieser Stelle ergibt sich eine Interdependenz zum ganzheitlichen Betreuungsansatz, denn die Potenzialeinschätzung wird umso genauer sein je detaillierter der Betreuer die Ziele und Pläne des Kunden analysiert hat. Es erscheint daher sinnvoll die Potenzial-erfassung an den intensiven Kundendialog im Rahmen des ganzheitlichen Strategiegespräches zu koppeln. ${ }^{205}$

\subsubsection{Kriterienscoring}

Nach der grundsätzlichen Auswahl der Klassifizierungsmerkmale soll nachfolgend das für die genossenschaftliche Bankengruppe entwickelte Scoringverfahren am Beispiel des Grobsegments Mittelstand erläutert werden.

\section{Kriterienbewertung}

Die Ausprägung der gewählten Segmentierungskriterien ist so zu beurteilen und zu gewichten, dass sich eine möglichst realitätsnahe Gesamteinschätzung für die jeweilige Portfolio-Dimension ergibt. Die Bandbreite der Scorewerte liegt zwischen null und vier Punkten. Die geeignete Abstufung der Auswahlmöglichkeiten ist - besonders bei qualitativen Merkmalen - von entscheidender Wichtigkeit. Dabei ist darauf zu achten,

$204 \mathrm{Vgl}$. kursiv gestellte Kriterien in Tabelle 13 in Anhang 11.

205 Vgl. Kapitel 7.4.1 
dass die Unterteilung transparent und verständlich ist. Die Besonderheiten sollen nun an einigen Beispielen veranschaulicht werden.

\begin{tabular}{|c|c|c|}
\hline Klassifizierungskriterien - Aktueller Status & & $\begin{array}{l}\text { Score } \\
\text { bzw. } \\
\text { Teilscore }\end{array}$ \\
\hline Intensitär der Bankverbindung & Gauptomkverbindung (3) & $3: 09$ \\
\hline Deckungsbeitrag der Betreuungseinheit p.a. & $19.36700 \mathrm{C}$ & $3 \% 37$ \\
\hline$\frac{\text { Deckungsbeitrag der Betreuungseinheit p.a. }}{\text { Firmenumsatz der Unternehmens gruppe }}$ & $\begin{array}{l}\text { DB II unter } 0.00 \text { EUR }(0.00) \\
\text { DB II } 0.00 \text { EUR bis 4.499.99 EUR (0.00-0.99) }\end{array}$ & 4,00 \\
\hline $\begin{array}{l}\text { Leistungsanalyse - Betriebliche Bedartsfelder } \\
\text { Bedarfsfeld Liquiditat und Zahlungsverkehr }\end{array}$ & $\begin{array}{l}\text { OB II 5000.00 EUR bis 9.999.99 EUR (1,00-1.99) } \\
\text { DB I| 10.000.00 EUR bis } 16.499 .99 \text { EUR }(2.00-2.99)\end{array}$ & \\
\hline $\begin{array}{l}\text { Zahlungsverkehr } \\
\text { - Kontokorrent } \\
\text { - Ausiandszahlungsverkehr } \\
\text { - eBanking }\end{array}$ & $\begin{array}{l}\text { DB II 15.000.00 EUR bis 19.999.99 EUR (3.00-3.99) } \\
\text { DB II ab 20.000.00 EUR (4.00) }\end{array}$ & $\frac{4,30}{1.32}$ \\
\hline
\end{tabular}

Abbildung 18: Kriterium Deckungsbeitrag - Merkmalsausprägung ${ }^{206}$

Der Deckungsbeitrag der Betreuungseinheit p.a. wird über bankinterne Informationssysteme für die Segmentierung bereitgestellt. In einem Kommentarfeld ist dabei aufgeschlüsselt, wie sich der Scorewert errechnet. Die Punktverteilung orientiert sich an den Ergebnissen der zeb/Firmenkundenstudie 2004, die geeignete Benchmarks für die Einschätzung des aktuellen Ergebnisbeitrags liefert. ${ }^{207}$ Der entsprechende Betrag wird auf zwei Dezimalstellen genau in einen Punktwert überführt. ${ }^{208}$

Die Leistungsanalyse folgt einer anderen Systematik: Die unterschiedlichen Produkte und Dienstleistungen wurden dazu in Nutzungsgruppen zusammengefasst. Innerhalb dieser Einheiten galt es im Rahmen von Segmentierungsworkshops die relative Wichtigkeit der einzelnen Bankleistungen so festzulegen, dass sich aggregiert 100\% ergeben. Die Nutzungsgruppen wurden dabei im betrieblichen Bereich auf Basis der Leistungsgruppen und in der privaten Sphäre auf Basis der Bedarfsfelder gebildet. ${ }^{209}$ Die Auswertung der Leistungsnutzung liefert eine zentrale Kundendatenbank, wobei in der Segmentierung lediglich abgebildet wird, ob das Produkt oder die Dienstleistung in Anspruch genommen wird oder nicht. Eine Inanspruchnahme sämtlicher Bank-

206 Eigenentwickeltes Segmentierungstool

207 Im Rahmen einer empirischen Analyse wurden in unterschiedlichen Unternehmensgrößenklassen DB-II-Zielwerte (Zins- und Provisionserlöse ./. Standardrisikokosten) errechnet, vgl. hierzu detailliert Käser, Burkhard et al. (2004), S. 13 f..

208 Die Errechnung des Scorewerts des Kriteriums Deckungsbeitrag kann demnach mathematisch wie folgt abgebildet werden:

Score $(\mathrm{DBII})=\left\{\begin{aligned} 0, & \text { falls } \mathrm{ABII}=0 \\ \frac{4}{20.000} \cdot \mathrm{DBII}, & \text { falls } 0<\mathrm{DBII} \leq 20.000 \\ 4, & \text { falls DBII }>20.000\end{aligned}\right.$

209 Die zusätzliche Abstufung in der betrieblichen Sphäre wird aufgrund des großen Umfangs des Leistungsportfolios vorgenommen. Zur vertrieblichen Nutzung der Detailinformationen vgl. Kapitel 9 
leistungen innerhalb einer Nutzungsgruppe entspricht dem Scorewert 4, dabei werden die relativen Wichtigkeiten der Leistungen in eine entsprechende Teilscore transformiert und aufsummiert. ${ }^{210}$

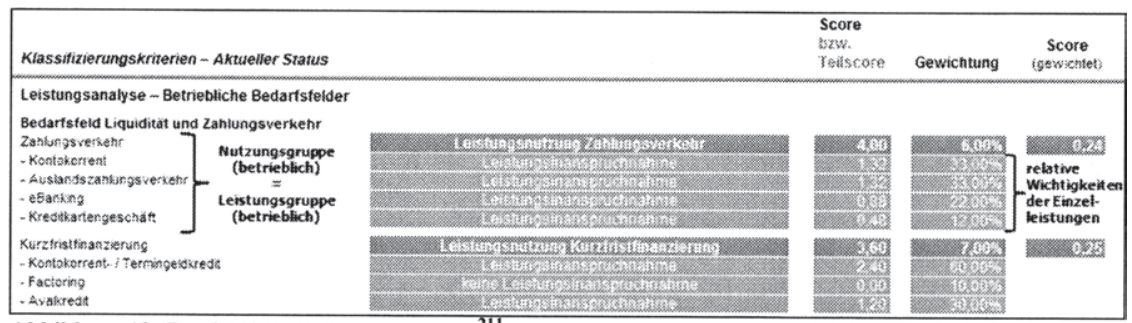

Abbildung 19: Betriebliche Leistungsanalyse ${ }^{211}$

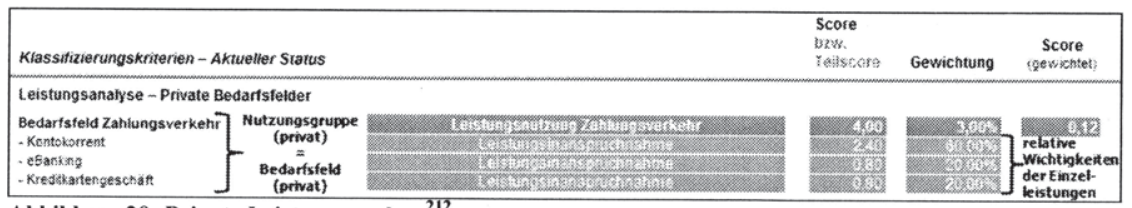

Abbildung 20: Private Leistungsanalyse ${ }^{212}$

Die Abgrenzung von quantitativen Umfeldfaktoren ist für die Entwicklung des Branchenumsatzes durch das BVR-Branchen special weitgehend vorgegeben und für die Scorewerte des $K f W$-ifo-Mittelstandsbarometers, der regionalen Kaufkraftkennziffer und der Sparquote der privaten Haushalte leicht abzuleiten. ${ }^{213}$

An den weichen Segmentierungsfaktoren sind jeweils Kommentarfelder hinterlegt, die ein einheitliches Verständnis, sowie eine Abgrenzung der Merkmalsausprägungen ermöglichen (vgl. Abbildung 21)

\section{Kriteriengewichtung}

Die Klassifizierungsfaktoren fließen aufgrund von sachlogischen Überlegungen gewichtet in die Gesamtbewertung der Portfolio-Dimensionen ein. In den Vorkapiteln wurde bereits mehrfach erläutert, dass die Bedarfsorientierung ein zentrales Element einer ganzheitlichen Betreuungsphilosophie darstellt. Somit muss dieser Aspekt durch die Gewichtung in beiden Dimensionen in den Vordergrund treten. Weitere Kriterien

$210 \mathrm{Vgl}$. Abbildung 19 und 20. In der Praxis ist zusätzlich zu berücksichtigen, dass bestimmte Bankleistungen sich auch untereinander ausschließen können. In so einem Fall muss der Maximalwert von 4 in der jeweiligen Nutzungsgruppe auch dann erreichbar sein, wenn nicht alle Leistungen in Anspruch genommen werden. Da eine solche Bedingung in einer Datenbankabfrage relativ einfach zu integrieren ist, wird der Sachverhalt an dieser Stelle nicht weiter vertieft.

211 Eigenentwickeltes Segmentierungstool

212 Eigenentwickeltes Segmentierungstool

$213 \mathrm{Vgl}$. Abbildung 44 und 45 in Anhang 12 
von hervorgehobener Wichtigkeit stellen der gegenwärtige und zukünftige Deckungsbeitrag der Betreuungseinheit dar.

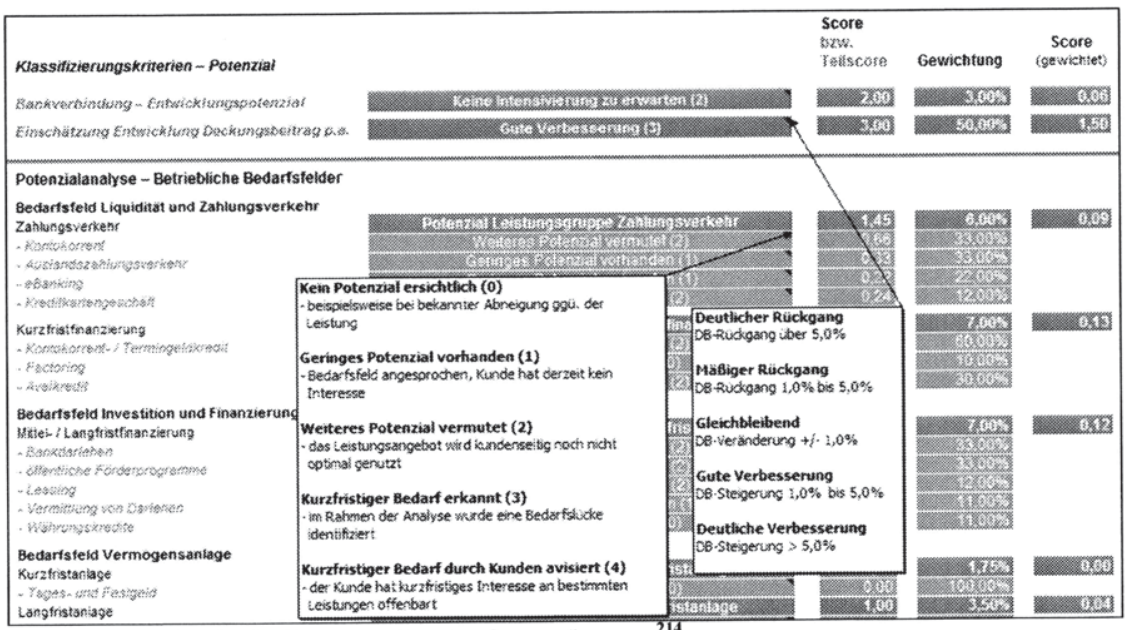

Abbildung 21: Qualitative Kriterien: Merkmalsausprägungen ${ }^{21}$

Im vorliegenden Segmentierungsansatz wurden auf Basis von Vertriebsüberlegungen und aufgrund von Erfahrungswerten, die Merkmale jeweils mit einer prozentualen Gewichtung belegt. Die Kriteriengewichtungen der einzelnen Portfolio-Dimensionen musste dabei $100 \%$ ergeben. Eine solche Gewichtungsstruktur sollte langfristig angelegt sein und nur bei Änderungen der Vertriebsphilosophie modifiziert werden. Außerdem hat die Gewichtung grobsegmentspezifisch erfolgen.

Aus der Multiplikation von Scorewert und Gewichtung errechnet sich eine gewichtete Score. Die gewichteten Ergebnisse ergeben in der dimensionsbezogenen Addition einen Gesamtpunktwert. Setzt man diesen ins Verhältnis zur Maximalscore erhält man eine Bewertungsziffer zwischen 0 und 100. Der gesamte Bewertungsvorgang wird für den Firmenkundenbetreuer in einem Profil veranschaulicht. So werden kriterienspezifische Tendenzen erkennbar und eine Transparenz des Segmentierungsprozesses erreicht. ${ }^{215}$

\subsubsection{Portfolio-Positionierung und Segmentierungsstufen}

Um hinreichend große Kundengruppen zu erhalten wird das Portfolio analog zur BCG-Matrix und zum Ansatz von SCHMOLL in vier Felder aufgeteilt. Die Kundenengagements sind in den Stufen Star, Potential, Cash Cow und Standard im Portfolio wie folgt kategorisiert:

214 Eigenentwickeltes Segmentierungstool

215 Eine grafische Darstellung erfolgt in ein einem Bewertungsbereich, vgl. hierzu die Abbildungen 46 bis 49 in Anhang 12. 


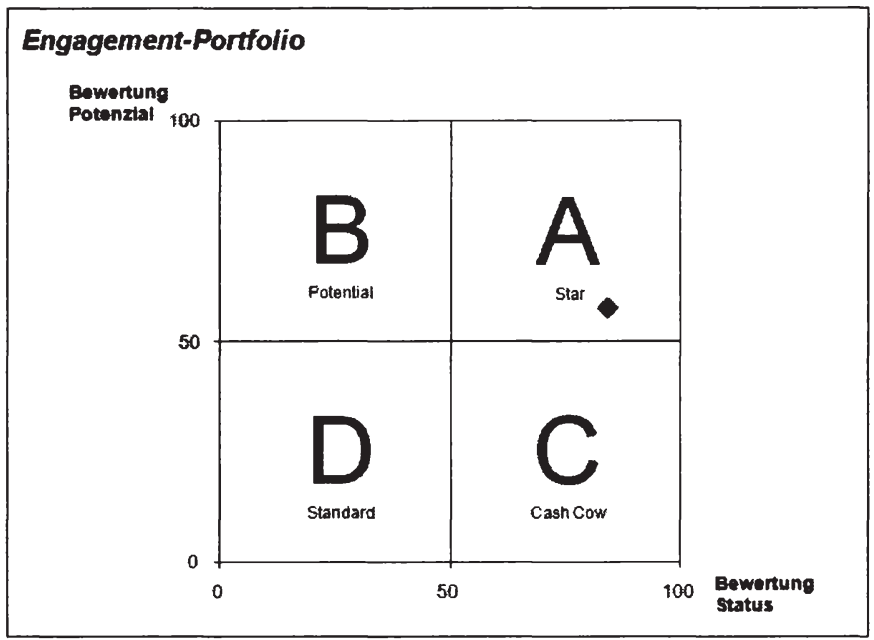

Abbildung 22: Engagement-Portfolio ${ }^{216}$

Hinsichtlich der daraus ableitbaren segmentspezifischen Betreuungsstrategien wird an dieser Stelle auf Kapitel 9 verwiesen. ${ }^{217}$

Die Positionierung des Firmenkundenengagements im Portfolio erfolgt, indem die im Scoring-Verfahren ermittelten Bewertungsziffern in das Koordinatensystem eingetragen werden. Die Dimensionsachsen sind dabei dem Wertebereich der Bewertungsziffern angepasst und unterteilen das Portfolio ab einem Wert von 50, so dass sich die vorgenannten vier Segmentierungsstufen ergeben.

\subsection{Ressourcenbindung und Risikosituation}

Das soeben vorgestellte Firmenkunden-Portfolio klassifiziert die Engagements nach Status und Potenzial und unter besonderer Berücksichtigung von Bedarfssituation und -perspektive. Die aktuelle Betreuungsintensität und die Risikosituation finden in der Darstellung keine Berücksichtigung, da es nach Ansicht des Verfassers sinnvoll ist diese beiden Komponenten gesondert zu betrachten.

\subsubsection{Das Ressourcen-Portfolio}

Die Betreuungsintensität stellt dabei eine Steuerungsgröße dar, die basierend auf dem Ergebnis der Feinsegmentierung vorgegeben werden soll. Daher erscheint es interessant, die aktuelle Betreuungsintensität im Vergleich zu den Dimensionsergebnissen zu

216 Eigenentwickeltes Segmentierungstool

217 In Kapitel 9 soll eine Verknüpfung des Feinsegmentierungsansatzes aus Kapitel 7 mit den Ergebnissen der Analyse der Kundenanforderungen aus Kapitel 8 erfolgen. 
beurteilen. Hierzu können Auswertungen aus dem Aktivitätencontrolling in die Segmentierung integriert werden. Beispielhaft kann dabei die Ressourcenverwendung nach den folgenden drei Gesprächstypen ermittelt und bewertet werden:

- Ganzheitliche Betreuungsgespräche,

- anlassbezogene Beratungen und

- Kurzkontakte.

Diese werden als Kriterien gewichtet in einem Ressourcen-Scoring zusammengefasst, um danach in einem Ressourcen-Portfolio abgebildet zu werden. ${ }^{218}$ Das Gesamtergebnis der Ressourcenbewertung wird dabei von der $\mathrm{x}$-Achse abgetragen, während die Bewertungsziffern der Dimensionen Status und Potenzial auf der y-Achse Berücksichtigung finden. Da es sich aufgrund der technischen Verfügbarkeit der Daten um quantitative Kriterien handelt bedeutet die zusätzliche Betrachtungsweise keinen Mehraufwand für den Betreuer, liefert aber sehr wohl einen Mehrwert: Es wird veranschaulicht, inwiefern die bisherige Betreuungsintensität zum aktuellen Engagementstatus und zur Potenzialeinschätzung passt. Für den Firmenkundenbetreuer wird sofort ersichtlich, ob die Betreuungsintensität einer Anpassung bedarf (vgl. Abbildung 23):

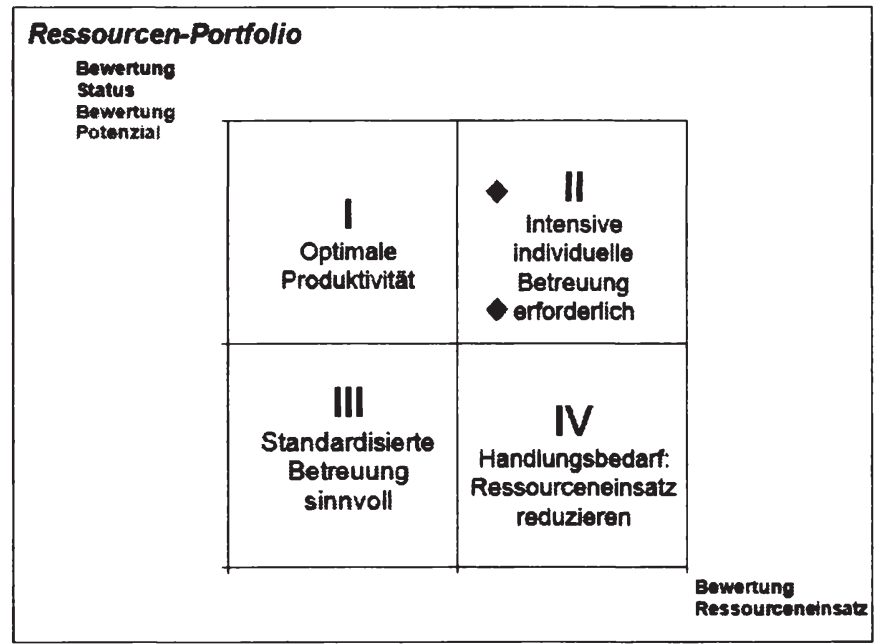

Abbildung 23: Ressourcen-Portfolio ${ }^{219}$

$218 \mathrm{Vgl}$. Abbildung 49 in Anhang 12 und Abbildung 23 auf der nachfolgenden Seite.

219 Eigenentwickeltes Segmentierungstool 


\subsubsection{Das Risiko-Portfolio}

Gerade im kreditintensiven Firmenkundengeschäft muss auch die Risikosituation gewürdigt werden. Diese sollte im Rahmen des Vetriebsmanagements jedoch nicht im Vordergrund stehen.

Mit der Einführung von Basel II wurde in den letzten Jahren Ratingsysteme für das mittelständische Firmenkundengeschäft in allen Institutsgruppen implementiert. Im Genossenschaftssektor wird hier das BVR-II-Rating eingesetzt, dass unter Einbezug quantitativer (aus dem Jahresabschluss) und qualitativer (strukturierter RatingFragebogen) Daten eine Einstufung der Bonität und die Ermittlung der Ein-JahresAusfallwahrscheinlichkeit des Firmenkunden intendiert. ${ }^{220}$ Es handelt bei dem System ebenfalls um ein Multifaktoren-Scoring, welches Teilscores für den Jahresabschluss und für die qualitativen Fragen vergibt. Diese Komponenten sollen nun zusammen mit der Besicherungsquote in einem zusätzlichen Risiko-Portfolio (vgl. Abbildung 24) zusammengefasst werden, so dass beurteilt werden kann, ob Risiko und Status in einem angemessenen Verhältnis stehen und, ob die Potenzialeinschätzung die Risikoposition rechtfertigt. ${ }^{221}$

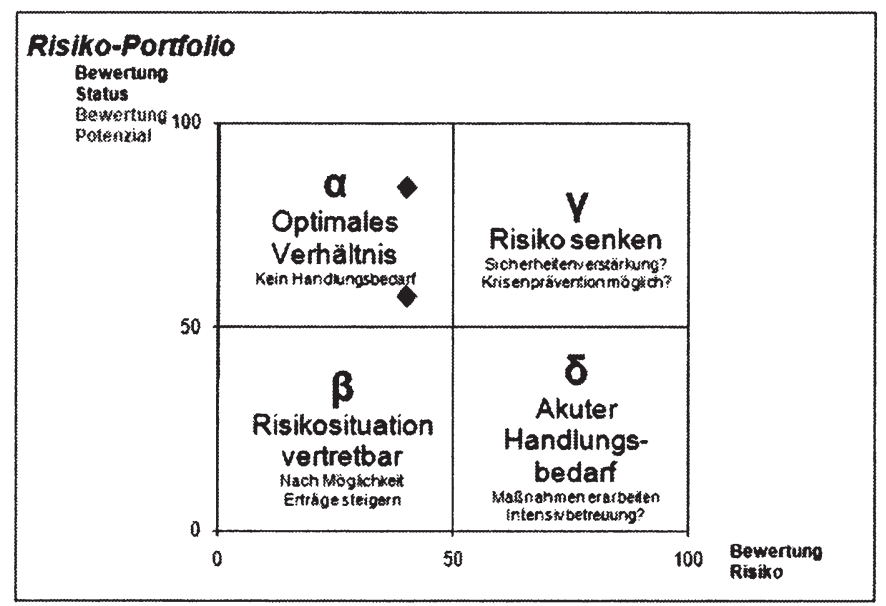

Abbildung 24: Risiko-Portfolio

\subsection{Konsequenzen für das Vertriebsmanagement}

Nachdem die Konzeption des Segmentierungsansatzes für Genossenschaftsbanken nun abgeschlossen ist, stellt sich die Frage wie die Klassifizierung idealerweise in die

${ }^{220} \mathrm{Vgl}$. für einen Überblick beispielsweise Klingbeil, Ernst / Yousefian, Patrick (2002), S. 28 f. oder Nowak, Helge (2002), S. 22 ff.

$221 \mathrm{Vgl}$. hierzu grafisch Abbildung 24 
Vertriebspraxis zu integrieren ist. Hierzu soll zunächst grafisch gezeigt werden, welche Interdependenzen entlang des Vertriebsprozesses bestehen, um darauf basierend eine idealtypische vertriebliche Nutzung der potenzialorientierten Segmentierung zu skizzieren.

Abbildung 25 zeigt vereinfachend die Wechselwirkungen der entwickelten Feinsegmentierungsmethodik, indem lediglich die Schnittstellen entlang des Vertriebsprozesses dargestellt wurden. Dabei sind alle Aspekte, die einen direkten oder indirekten Einfluss, auf die Feinsegmentierung haben, in schwarz hervorgehoben. In der Betrachtung wird zwischen der strategischen und operativen Ebene unterschieden.

Bevor der operative Regelkreis des um die Feinsegmentierung erweiterten Betreuungsprozesses erläutert wird, sind die strategischen Verflechtungen zu erklären:

Es wurde bereits angeführt, dass die Segmentierungskriterien grobsegmentspezifisch festzulegen sind. So haben Veränderungen der Grobsegmentstruktur zwangsläufig eine Überprüfung der Feinklassifizierungsmerkmale zur Folge, damit die Verfahren sich optimal ergänzen.

Die zweite strategische Einflussgröße ist in der Planung zu sehen. Im Firmenkundengeschäft wird häufig eine integrierte Top-down-/Bottom-up-Vertriebsplanung verwendet. ${ }^{222}$ Die Feinklassifizierung verbessert beide Seiten des Planungsprozesses, indem sie eine differenziertere Top-Down-Planung ermöglicht und den Firmenkundenbetreuern die operative Bottom-Up-Planung erleichtert. Durch die Feinsegmentierung wird das Kundenportfolio der Betreuer strukturiert. Somit können je Subsegment unterschiedliche Zielvorgaben festgelegt werden, die sich am aktuellen Status und Potenzial der Engagements orientieren. Die Betreuer haben mit den leistungsbezogenen Potenzialeinschätzungen eine strukturierte Planungsgrundlage, die auf einem ganzheitlichen Kundendialog beruht. ${ }^{223}$ Mehr Transparenz wird auch im Bereich der Risikosteuerung erreicht, da durch das Risiko-Portfolio festzustellen ist, wie sich die Ausfallrisiken auf die Subsegmente verteilen. Dies würde zum Beispiel eine unterschiedliche Risikolimitierung je Feinsegment denkbar machen.

Während die Grobsegmentierung als strategischer Rahmen der Marktbearbeitung fungiert, ist die Feinsegmentierung als Ausgangspunkt eines effizienten Betreuungsprozesses zu verstehen. Wie in Abbildung 25 veranschaulicht ergibt sich ein operativer Kreislauf der Kundenbetreuung, dessen idealtypischer Verlauf wie folgt aussieht.

222 Vgl. Krauß, Carsten (2006), ohne Seitenangabe

223 Die Jahresplanung verläuft leistungsgruppenorientiert, durch die Aufnahme der steuerungsrelevanten Leistungen in Feinsegmentierung können die Segmentierungsergebnisse je Betreuer zusammengefasst und in der Planung verwendet werden. 


\subsubsection{Wechselwirkungen der Kundensegmentierung im Vertriebsprozess}

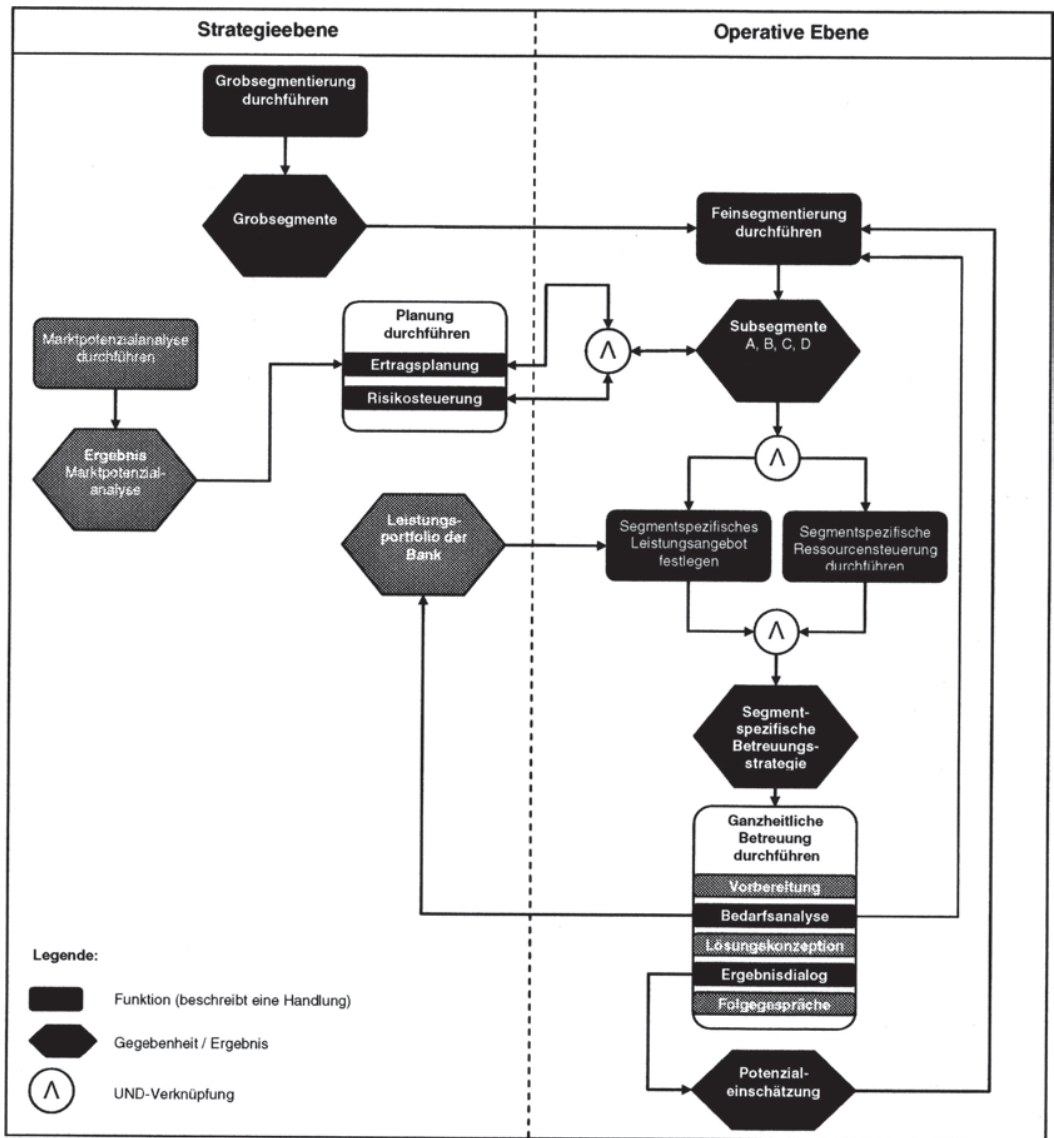

Abbildung 25: Regelkreis einer zweistufigen Kundensegmentierung ${ }^{224}$

Die für die Feinsegmentierung notwendigen qualitativen Informationen werden betreuerseitig im Rahmen des turnusgemäßen ganzheitlichen Strategiedialoges erhoben. Auf Basis der Gesprächsergebnisse erfolgt je Bedarfsfeld eine strukturierte Potenzialeinschätzung im Segmentierungstool. Diese vorläufige Bewertung kann bis zu einem jährlichen Segmentierungsstichtag überarbeitet und modifiziert werden. Zwei Wochen vor Beginn des Feinsegmentierungslaufes, wird zur Erledigung der noch nicht bearbeiteten und zur Prüfung der eingegebenen Segmentierungsbögen aufgerufen. Das Klassifizierungsergebnis wird operativ zum segmentspezifischen Zuschnitt des Leistungsangebotes und zur Priorisierung der Vertriebsaktivitäten

224 Eigene Darstellung. 
verwendet. ${ }^{225}$ Etwaige Bedarfe in einzelnen Leistungsfeldern können als Vertriebsimpulse in das jeweilige CRM-System überführt werden. Die Kriterienbewertung wird dann im nächsten Strategiegespräch wieder an die dann aktuelle Situation angepasst. Aufgrund der Tatsache, dass die Firmenkundenbetreuer gemäß VR-Finanzplan Mittelstand sowieso mindestens ein StrategieGespräch pro Jahr mit ihren Kunden führen und die Gesprächsnachbereitung bisher lediglich uneinheitlich erfolgt, bedeutet das Segmentierungsprozedere - bis auf die erstmalige Segmentierung - keinen nennenswerten Mehraufwand. Vielmehr wird die sowieso notwendige Festhaltung der Gesprächsergebnisse in eine vertrieblich weiterverwendbare Struktur gebracht.

\subsubsection{Validität der Segmentierungsergebnisse}

Die bisherigen Ausführungen zur Portfolio-Analyse haben verdeutlicht, dass es erforderlich ist, einen Weg zu finden mit den auftretenden Unsicherheiten und der Subjektivität der Methode umzugehen.

Die in Kapitel 6.3.2 vorgestellten Multifaktorenansätze können nicht ohne weiteres auf ihre Validität überprüft werden, da sie mit unterschiedlichen Faktoren für die Statusund die Potenzialdimension arbeiten. Aufgrund der gleichartigen Kriterienstruktur können die verwendeten Subsegmentzuordnungen - mit der in diesem Kapitel entwickelten Vorgehensweise - statistisch auf ihre Genauigkeit überprüft werden.

Dies kann nach Ablauf eines Segmentierungszeitraumes im Rahmen einer statistischen Abweichungsanalyse geschehen. So können die Segmentierungsergebnisse beispielsweise daraufhin überprüft werden, ob sich signifikante Ergebnisabweichungen auf bestimmte Betreuer konzentrieren, um so Fehleinschätzungen zu identifizieren. Unter Einsatz multivariater Analysemethoden wäre auch die Überprüfung der Merkmalsausprägungen und der Gewichtung denkbar. Allerdings bleibt abzuwarten, ob sich dieser Ansatz als Gütemaß trotz der Vielzahl ergebnisbeeinflussender Komponenten als praktikabel erweist. Falls dies nicht der Fall sein sollte, kann die Segmentierungshistorie dazu verwendet werden, einen statistischen Bereinigungsfaktor je Dimension zu ermitteln, der die tatsächliche Bewertungsziffer um einen durchschnittlichen Fehler bereinigt. Für eine solche Bereinigung, sollte allerdings eine mehrjährige Segmentierungshistorie zur Verfügung stehen, um aussagefähige Ergebnisse zu erhalten.

Die hier genannten Validierungsansätze sind jedoch nur als Ideen zu verstehen. Eine differenziertere Aussage kann erst getroffen werden, nachdem eine Segmentierungshistorie aufgebaut wurde. Die Datensätze sind dann mit mehreren statistischen Verfahren zu analysieren, um unter den eingesetzten Methoden die am besten Geeignete herauszufiltern.

225 Vgl. hierzu Kapitel 9 


\section{Anforderungsstrukturen im Firmenkundengeschäft - Eine empirische Analyse}

\subsection{Ziel der Erhebung}

In Kapitel 7.4.1 wurde ausgeführt, dass die Ergebnisse der Feinsegmentierung zur segmentspezifischen Zuordnung des Leistungsangebotes herangezogen werden können. In diesem Zusammenhang ist allerdings auch zu überprüfen, ob die Bedarfssituation durch Leistungsportfolio hinreichend abgedeckt werden kann. Daneben erscheint es interessant die Beziehungsmerkmale nach ihrer Relevanz für die Geschäftsbeziehung zu strukturieren, um die daraus resultierenden Erkenntnisse bei der Festlegung der Betreuungsressourcen zu berücksichtigen und diese im Vertrieb aktiv zu nutzen.

Dazu soll die Anforderungsstruktur der Firmenkunden sowohl bezogen auf das Leistungsportfolio als auf verschiedene Beziehungsmerkmale untersucht werden. Ziel ist es auf Basis einer empirischen Untersuchung Basis-, Leistungs- und Begeisterungsanforderungen im Firmenkundengeschäft zu identifizieren. Wie bereits in Kapitel 4.2 dargelegt ist die Kenntnis der Kundenanforderungen als notwendige Determinante einer erfolgreichen Kundenbindungsstrategie anzusehen. Insbesondere den Begeisterungskomponenten kommt dabei eine entscheidende Rolle zu, da ihre Erfüllung die Entstehung einer langfristig orientierten, belastbaren Geschäftsbeziehung ermöglicht. ${ }^{226}$

Im Kontext der Kundenbegeisterung wird häufig diskutiert, ob sich das Rollenverständnis des Firmenkundenbetreuers zur Erzielung einer begeisternden Differenzierungswirkung ändern muss. So schlagen mehrere Autoren vor, dass sich der Firmenkundenbetreuer zu einem unternehmerischen Ratgeber entwickeln müsse. ${ }^{227}$ Vor diesem Hintergrund soll diese These in der empirischen Analyse Berücksichtigung finden.

\subsection{Untersuchungsdesign}

\subsubsection{Wahl der Analysemethode}

$\mathrm{Da}$ in der vorliegenden Arbeit dem Kano-Modell gefolgt wird, ist ein Verfahren auszuwählen, welches in der Lage ist die Drei-Faktor-Struktur der Kundenanforderungen abzubilden. Im Wesentlichen sind hier die an die Modellbezeichnung angelehnte Kano-Methode und das Importance Grid zu nennen. Nach einer überblicksartigen

226 Die Erfüllung von Begeisterungsanforderungen ruft positive Emotionen hervor, die die Intensität der Kundenbindung in den beiden Bindungsdimensionen der Ge- und Verbundenheit begünstigen, vgl. hierzu u.a. Kapitel 4.2.2

227 Vgl. u.a. Schmoll, Anton (2006), S. 89 oder Schmidt, Thomas (2001), S. $151 \mathrm{ff}$. 
Vorstellung der beiden Methoden, soll das hier zur Anwendung kommende Importance Grid näher erläutert werden.

\section{Kano-Methode}

Im Rahmen des Kano-Verfahrens wird zur Klassifizierung der Anforderungsstruktur ein Fragebogen eingesetzt, der die Beurteilung der Leistungseigenschaften jeweils durch eine funktionale und eine dysfunktionale Form der Frage ermittelt. ${ }^{228}$ Die erste Frage bezieht sich auf die Reaktion des Befragten bei Vorhandensein der Leistungskomponente, die zweite auf ihr Nichtvorhandensein. ${ }^{229}$ Auf den Untersuchungskontext bezogen könnte eine Fragenkombination beispielsweise wie folgt aussehen:

- funktional:

„Wenn Ihre Hausbank Sie bei der Liquiditätsplanung unterstützt, wie denken Sie darüber?“

- dysfunktional:

„Wenn Ihre Haubank Sie bei der Liquiditätsplanung nicht unterstützt, wie denken Sie darüber?"

Dem Befragten stehen jeweils fünf Auswahlmöglichkeiten zur Verfügung, die bei allen Fragen identisch sind. Die Antworten werden über eine Auswertungsmatrix in eine Ergebnistabelle überführt, welche eine Zuordnung der Leistungseigenschaften zu den Anforderungstypen ermöglicht.

Gegen eine Anwendung der Kano-Methode im Rahmen einer Unternehmensbefragung im Firmenkundengeschäft bestehen vor allem Praktikabilitätsbedenken. ${ }^{230}$ So würde für die Untersuchung der Leistungs- und Beziehungsmerkmale ein sehr langer Fragebogen benötigt. Des Weiteren ist davon auszugehen, dass sich dem Befragten die Sinnhaftigkeit der Fragetechnik nicht erschließt. Dieser Vermutung verstärkt sich nochmals, wenn man berücksichtigt, dass das Leistungsportfolio von Kreditinstituten eine gewisse Abstraktheit aufweist. So ist der Firmenkundenbetreuer gefordert auch latente Bedarfe zu erkennen, die dem Kunden als solche noch nicht bewusst sind. Aufgrund der Komplexität und des Abstraktionsniveaus kann der Einsatz der Methodik als kaum für eine schriftliche Befragung geeignet eingestuft werden und wird daher nicht weiter verfolgt. ${ }^{231}$

\footnotetext{
228 Dabei ist die dysfunktionale Formulierung als Negierung der funktionalen Frage zu verstehen.

230 Da aufgrund der problematischen praktischen Anwendung von einer Verwendung der KanoMethode abgesehen wird, wird an dieser Stelle auf eine detaillierte Darstellung der Ergebnisauswertung verzichtet.

231 Eine kritische Würdigung der Methodik findet sich außerdem u.a. bei Sauerwein, Elmar (2000) und Bailom, Franz et al. (1996)
} 


\section{Importance Grid}

Eine relativ neuartige Methode zur Ermittlung der Anforderungsstruktur stellt das von VAVRA entwickelte Importance Grid dar. Es wird dabei von der Prämisse ausgegangen, dass zwischen einer expliziten und einer impliziten Wichtigkeit unterschieden werden kann. Dabei erfasst die explizite Wichtigkeit eine direkt artikulierte Wichtigkeitseinschätzung des Kunden, während die implizite, indirekte Wichtigkeit die tatsächliche Zufriedenheits- und Emotionswirkung beschreibt. ${ }^{232}$

Die drei Anforderungstypen werden in Tabelle 5 den unterschiedlichen Wichtigkeitsdimensionen zugeordnet:

\begin{tabular}{|l|l|l|}
\hline Anforderungstyp & Explizite Wichtigkeit & Implizite Wichtigkeit \\
\hline Basisanforderungen & $\begin{array}{l}\text { werden bei direkter Nachfrage als } \\
\text { sehr wichtig eingestuft, da sie als } \\
\text { Grundbedürfnis angesehen werden }\end{array}$ & $\begin{array}{l}\text { - geringe Emotions- und } \\
\text { Zufriedenheitswirkung, führen } \\
\text { lediglich zu einem Status der } \\
\text { Nicht-Unzufriedenheit }\end{array}$ \\
\hline Leistungsanforderungen & $\begin{array}{l}\text { können als wichtig oder unwichtig } \\
\text { beurteilt werden } \\
\text { - Unterschiede werden im } \\
\text { Wettbewerbsvergleich direkt } \\
\text { wahrgenommen }\end{array}$ & $\begin{array}{l}\text { Zufriedenheitswirkung: } \\
\rightarrow \text { bei hoher expliziter } \\
\text { Wichtigkeit, hohe Wirkung } \\
\text { bei niedriger expliziter Wich- } \\
\text { tigkeit, niedrige Wirkung }\end{array}$ \\
\hline Begeisterungs-anforderungen & $\begin{array}{l}\text { werden als eher unwichtig } \\
\text { eingeschätzt, da sie nicht } \\
\text { erwartet werden }\end{array}$ & $\begin{array}{l}\text { hohe Emotions- und } \\
\text { Zufriedenheitswirkung, führen } \\
\text { zu einer Kundenbegeisterung }\end{array}$ \\
\hline
\end{tabular}

Tabelle 5: Explizite und implizite Wichtigkeit von Kundenanforderungen ${ }^{233}$

Das Verfahren bildet auf diese Weise die grundsätzlichen Annahmen des KanoModells ab, was nachfolgend exemplarisch erläutert wird:

Ein Firmenkunde, der nach der Wichtigkeit einer schnellen und ordnungsgemäßen Ausführung seiner Überweisungsaufträge gefragt wird, wird die Leistungserfüllung als Grunderwartung an die Geschäftsbeziehung ansehen und somit ihre Erfüllung als sehr wichtig einstufen. Allerdings führt das Vorhandensein der Leistungseigenschaft lediglich zu einem Status der Nicht-Unzufriedenheit und weist daher eine geringe implizite Wichtigkeit auf. Ein umgekehrtes Verhältnis wird nun bei bankseitigen, betriebswirtschaftlichen Beratungsleistungen vermutet. Das Angebot dieser Leistungen müsste daher explizit in Relation zu anderen Bankleistungen eher als unwichtig eingeschätzt werden. Implizit sollte jedoch eine hohe Begeisterungswirkung nachzuweisen sein. Die Anforderungsstruktur kann demnach in einem Wichtigkeitsgitter - dem Importance Grid - visualisiert werden (vgl. Abbildung 26).

232 Vgl. Matzler, Kurt et al. (2006), S. 304

2.33 In Anlehnung an die verbalen Erläuterungen bei Matzler, Kurt et al. (2006), S. 304 f. 


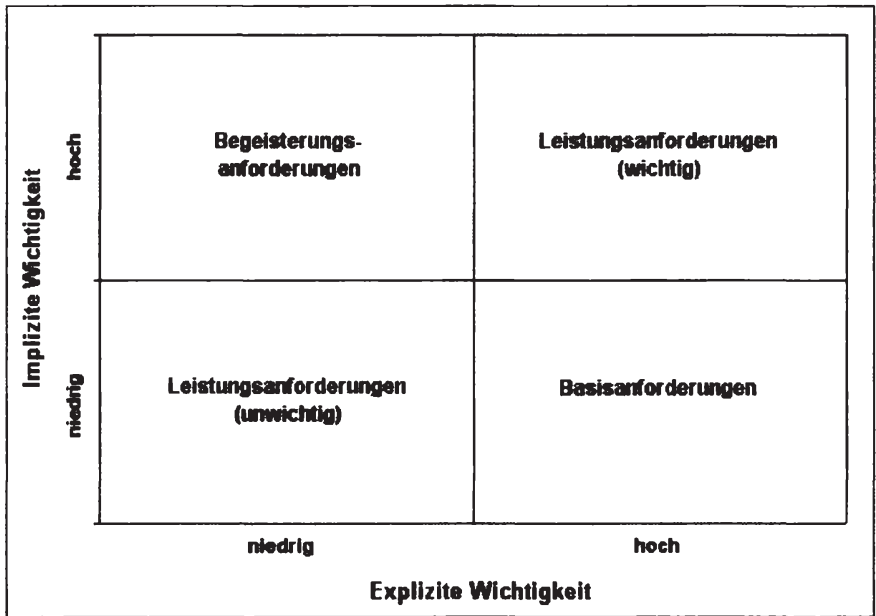

Abbildung 26: Das Importance Grid ${ }^{234}$

Zur Positionierung der Kundenanforderungen in diesem Wichtigkeitsportfolio ist die Messung der Wichtigkeitsdimensionen erforderlich. Dazu ist die explizite Wichtigkeit skaliert abzufragen, während die implizite Wichtigkeit indirekt errechnet werden kann. Hierzu wird die Gesamtzufriedenheit des Kunden erfragt und mit den Einzelzufriedenheiten der interessierenden Leistungskomponenten multipel linear regressiert. ${ }^{235}$ Die so ermittelten Werte müssen dann zweidimensional abgebildet werden. Zunächst ist ein geeignetes Skalenniveau festzulegen. Die Methodik sieht dabei vor den niedrigsten Ergebniswert als Skalenbeginn und das arithmetische Mittel der Attribute zur Unterteilung der Achsen in niedrig und hoch vorzusehen. ${ }^{236}$ Die deskriptiv ermittelten durchschnittlichen Einzelwichtigkeiten werden auf der horizontalen Achse abgetragen. Eine Einstufung der impliziten Wichtigkeit erfolgt indem die standardisierten Regressionskoeffizienten auf der vertikalen Achse festgehalten werden. ${ }^{237}$

234 In Anlehnung an Vavra, Terry Gwyn (1997), S. 385. Die Kategorisierung der Leistungseigenschaften in wichtig und unwichtig, ergibt sich aus der Überlegung, dass Leistungsanforderungen als Funktion der Kundenzufriedenheit zu verstehen sind. Das Vorhandensein eines aus Kundensicht weniger entscheidenden Attributes führt demnach auch nur zu einer marginalen Erhöhung der Zufriedenheit. Vgl. Matzler, Kurt et al. (2006), S. 304 f.. Zur Methodik der Regressionsanalyse, vgl. ausführlich Backhaus, Klaus et al. (2006), S. $45 \mathrm{ff}$.

236 Vgl. Matzler, Kurt et al. (2006), S. 307

237 Durch eine Standardisierung der Regressionskoeffizienten wird eine Bereinigung unterschiedliche Messdimensionen der Variablen erreicht, vgl. Backhaus, Klaus et al. S.62 f.. 
Da das Verfahren die Struktur des Kano-Modells sachlogisch plausibel abbildet, die Modellprämissen empirisch bestätigt wurden und die zu erhebenden Messgrößen aufgrund ihrer leichten Verständlichkeit gut in einer schriftlichen Befragung eingesetzt werden können, orientiert sich das Fragebogendesign an den Anforderungen des Importance Grid. ${ }^{238}$

Bevor darauf im nächsten Abschnitt näher eingegangen wird, soll auch das Importance Grid einer kritischen Würdigung unterzogen werden. Zunächst ist hier anzuführen, dass es aufgrund der Neuartigkeit der Methode noch nicht hinreichend empirisch validiert ist. ${ }^{239}$ Dies ist vor allem auf eine relativ geringe Anzahl der Untersuchungen zurückzuführen. ${ }^{240}$ Ein weiterer Kritikpunkt liegt in der Einteilung der Quadranten anhand der Mittelwerte, welche auf den ersten Blick relativ willkürlich erscheint. ${ }^{241}$ Werden allerdings Attribute homogenen Typs betrachtet, kann der Sachverhalt logisch begründet werden, indem argumentiert wird, dass der Mittelwert aller Merkmale auch eine mittlere ex- bzw. implizite Wichtigkeit ausdrückt. Das Zuordnungsergebnis steht also in Relation zur betrachteten Merkmalsgruppe. ${ }^{242}$ In der empirischen Vorgehensweise kann dieser Problematik konzeptionell begegnet werden, indem ein separates Importance Grid je Attributtyp erstellt wird.

\subsubsection{Fragebogendesign und Struktur der Analyse}

In diesem Teilkapitel soll nun der Aufbau des in der Untersuchung eingesetzten Fragebogens vorgestellt werden.

Für die Analyse der Anforderungsstruktur im Firmenkundengeschäft, galt es zunächst geeignete, untersuchungsrelevante Attribute zu identifizieren. Die Merkmalsauswahl erfolgte dabei in den Attributkategorien Dienstleistungen und Beziehungsmerkmale.

Die Selektion orientierte sich im Bereich Dienstleistungen an der im VR-Finanzplan Mittelstand vorgesehenen Bedarfsfeldstruktur. Der Begriff Unternehmensmanagement wurde zur besseren Verständlichkeit durch die Bezeichnung Betriebswirtschaftliche Beratung ersetzt. Unter dieser Rubrik wurden sodann sowohl banknahe als darüber hinausgehende betriebswirtschaftliche Beratungsdienstleistungen zusammengefasst. Auf diese Weise kann die Affinität zu derartigen Leistungsangeboten überprüft werden.

\footnotetext{
${ }^{238}$ Zur empirischen Überprüfung der Prämissen und Kausalzusammenhänge, vgl. Matzler, Kurt / Sauerwein, Elmar (2002), S. $316 \mathrm{ff}$.

240 Somit kann die vorliegende Arbeit evtl. auch einen Beitrag zur Validierung der Methode liefern. Hierzu ist allerdings ein statistischer Vergleich mit anderen Methoden erforderlich. Dieser kann jedoch aufgrund des zur Verfügung stehenden Rahmens nicht Gegenstand dieser Arbeit sein. Vgl. Matzler, Kurt et al. (2006), S. 309

242 Dies liegt daran, dass jedes in die Analyse einbezogene Merkmal Einfluss auf den Mittelwert hat.
} 
Bei den Beziehungsmerkmalen wurden die Vorschläge von SEGBERS aufgegriffen, der im Rahmen seiner Arbeit eine theoretische Zuordnung der Anforderungsdimensionen im Kontext von Hausbankbeziehungen vorgenommen hat. Die getroffene Einteilung soll hier empirisch validiert werden. ${ }^{243}$

Um die Importance Grid-Methode anwenden zu können müssen sowohl die Wichtigkeit und Zufriedenheit der einzelnen Attribute als auch die Gesamtzufriedenheit gemessen werden. Für die Beurteilung wurde jeweils eine Skala von 1 bis 7 vorgegeben. ${ }^{24}$ Neben der Hauptintention der Identifikation von Basis-, Leistungs- und Begeisterungsanforderungen liegt ein weiterer Schwerpunkt in der genaueren Betrachtung der Erwartungen im Bedarfsfeld Betriebswirtschaftliche Beratung. Hierzu wurde direkt abgefragt, inwiefern sich die Unternehmen ein stärkeres Engagement bei den einzelnen Beratungsleistungen wünschen, wie sie die aktuelle Kompetenz der Firmenkundenbetreuer in dem jeweiligen Bereich einschätzen und ob die Betreuung unter Hinzuziehung eines Spezialisten erfolgen sollte. Auf diese Weise soll untersucht werden, ob eine Interdependenz zwischen der Kompetenzvermutung und der Erwartungshaltung besteht. Daneben werden noch einige andere kontextrelevante Sachverhalte überprüft. Einen Überblick auf die daraus abgeleitete Struktur der Analyse findet sich in Tabelle 6:

\begin{tabular}{|c|c|c|}
\hline Fragebogenabschnitt & Untersuchter Sachverhalt/Zusammenhang & $\begin{array}{l}\text { Angewandte } \\
\text { Methode }\end{array}$ \\
\hline $\begin{array}{l}\text { Teil 1: } \\
\text { Allgemeines }\end{array}$ & $\begin{array}{l}\text { Struktur der Stichprobe } \\
\text { - Branchen- / Umsatzinformationen } \\
\text { - Eigenkapitalstruktur } \\
\text { - Anzahl der Bankverbindungen } \\
\text { - Hausbank } \\
\text { - Dienstleisterübergreifende Gesamtzufriedenheit }\end{array}$ & Deskriptive Analyse \\
\hline $\begin{array}{l}\text { Teil 2: } \\
\text { Leistungsangebot im } \\
\text { Firmenkundengeschäft }\end{array}$ & $\begin{array}{l}\text { Mehrdimensionale Analyse des Leistungsangebotes } \\
\text { - Proaktivität der Dienstleister } \\
\text { - Dienstleisterübegreifende } \\
\text { Leistungsinanspruchnahme } \\
\text { - Zusammenhang zwischen expliziter und impliziter } \\
\text { Wichtigkeit }\end{array}$ & $\begin{array}{l}\text { Deskriptive Analyse } \\
\text { Importance Grid, } \\
\text { u.a. }\end{array}$ \\
\hline $\begin{array}{l}\text { Teil 3: } \\
\text { Anforderungen an die } \\
\text { Geschäftsbeziehung }\end{array}$ & $\begin{array}{l}\text { Mehrdimensionale Analyse der Beziehungsmerkmale } \\
\text { - Zusammenhang zwischen expliziter und impliziter } \\
\text { Wichtigkeit } \\
\text { - Bindungscharakter der Bindungsdimensionen }\end{array}$ & $\begin{array}{l}\text { Importance Grid, } \\
\text { u.a. } \\
\text { Regressionsanalyse }\end{array}$ \\
\hline $\begin{array}{l}\text { Teil 4: } \\
\text { Betriebswirtschaftliche } \\
\text { Beratung }\end{array}$ & $\begin{array}{l}\text { Interdependenzen im Kontext von } \\
\text { Beratungsleistungen } \\
\text { - Zusammenhang zwischen Erwartung und } \\
\text { Kompetenz } \\
\text { - Einschätzung des Preis-Leistungs-Verhältnisses }\end{array}$ & $\begin{array}{l}\text { Korrelationsanalyse } \\
\text { Deskriptive Analyse }\end{array}$ \\
\hline
\end{tabular}

Tabelle 6: Struktur der empirischen Analyse

243 Zur Einteilung der Anforderungsdimensionen nach SEGBERS, vgl. hierzu Anhang 4.

$244 \mathrm{Vgl}$. hierzu die Fragen 1.6, 2.2 und 2.3 im Fragebogen in Anhang 13 


\subsubsection{Angaben zur Datenerhebung}

Bevor die Ergebnisauswertung erläutert wird sollen hier noch einige Informationen zum Ablauf der Befragung und zur Stichprobenstruktur folgen:

Anhand eines Pre-Test bei zehn Unternehmenskunden unterschiedlicher Branchenzugehörigkeit und Größe, wurde der Fragebogen auf Verständlichkeit und inhaltliche Plausibilität getestet. Die Vorstudie ergab keinen Modifikationsbedarf.

Der Fragebogen wurde daraufhin an 1.321 Unternehmenskunden von Genossenschaftsbanken mit der Bitte um schriftliche Teilnahme oder Internetbeteiligung versandt. Der Befragungszeitraum erstreckte sich vom 08. November 2006 bis zum 08. Dezember 2006. Bis zum Endtermin konnte ein Rücklauf von 148 Fragebögen erzielt werden, was einer Rücklaufquote von 11,2\% entspricht. Nach Sichtung des Datenmaterials mussten 36 Fragebögen aufgrund von Falschausfüllungen eliminiert werden.

Die Struktur der verbleibenden Stichprobe von 112 Datensätzen ist wie folgt auf die Grobsegmente verteilt:

\begin{tabular}{|l|r|r|l|r|r|}
\hline $\mathbf{1 . 2}$ Umsatzstruktur & absolut & relativ & & absolut & relativ \\
\hline Gewerbekunden & 28 & $25,00 \%$ & unter $250.000 €$ & 14 & $12,50 \%$ \\
\cline { 4 - 7 } & & & $250.000 €$ bis unter $500.000 €$ & 14 & $12,50 \%$ \\
\hline Mittelstand & 58 & $51,79 \%$ & $500.000 €$ bis unter $1.000 .000 €$ & 15 & $13,39 \%$ \\
\cline { 4 - 7 } & & & $1.000 .000 €$ bis unter $2.500 .000 €$ & 30 & $26,79 \%$ \\
\cline { 4 - 7 } & & $26,500.000 €$ bis unter $5.000 .000 €$ & 13 & $11,61 \%$ \\
\hline Oberer Mittelstand & 26 & $5.000 .000 €$ bis unter $10.000 .000 €$ & 7 & $6,25 \%$ \\
\hline Gesamtwert & & & $10.000 .000 €$ bis unter $50.000 .000 €$ & 14 & $12,50 \%$ \\
\hline
\end{tabular}

Tabelle 7: Umsatzstruktur der Stichprobe

Es wird sich zeigen, dass der Stichprobenumfang für eine separate Betrachtung der beiden Grobsegmente zu klein ist, die Repräsentativität der Gesamtstichprobe ist jedoch gegeben.

Hinsichtlich der Sektorenzugehörigkeit sind Produktions-, Handels- und Dienstleistungsunternehmen mit einem Anteil zwischen $26 \%$ und $29 \%$ am Stärksten vertreten. ${ }^{245}$ Die Firmenkunden unterhalten dabei im Durchschnitt zwei Bankverbindungen. Etwa 90\% der Befragten gaben an ihre Hausbankverbindung bei einer Genossenschaftsbank zu unterhalten.

$245 \mathrm{Vgl}$. hierzu grafisch Abbildung 50 in Anhang 14 


\subsection{Anwendung der Importance Grid-Methode}

\subsubsection{Festlegung der Untersuchungshypothesen}

Die Analyse der Kundenanforderungen hat, da für das mittelständische Firmenkundengeschäft noch keine empirischen Befunde in diesem Bereich vorliegen, grundsätzlich zunächst explorativen Charakter. Daneben sollen jedoch bankbetriebswirtschaftliche Fragestellungen betrachtet werden. Im Fokus stehen dabei die Begeisterungswirkung von betriebswirtschaftlichen Beratungsleistungen und die von SEGBERS vorgeschlagene Merkmalskategorisierung. Die Untersuchung der Anforderungsstruktur bedarf gemäß den Vorgaben des Importance Grid eines mehrstufigen Hypothesentests, wie in Abbildung 27 beispielhaft veranschaulicht.

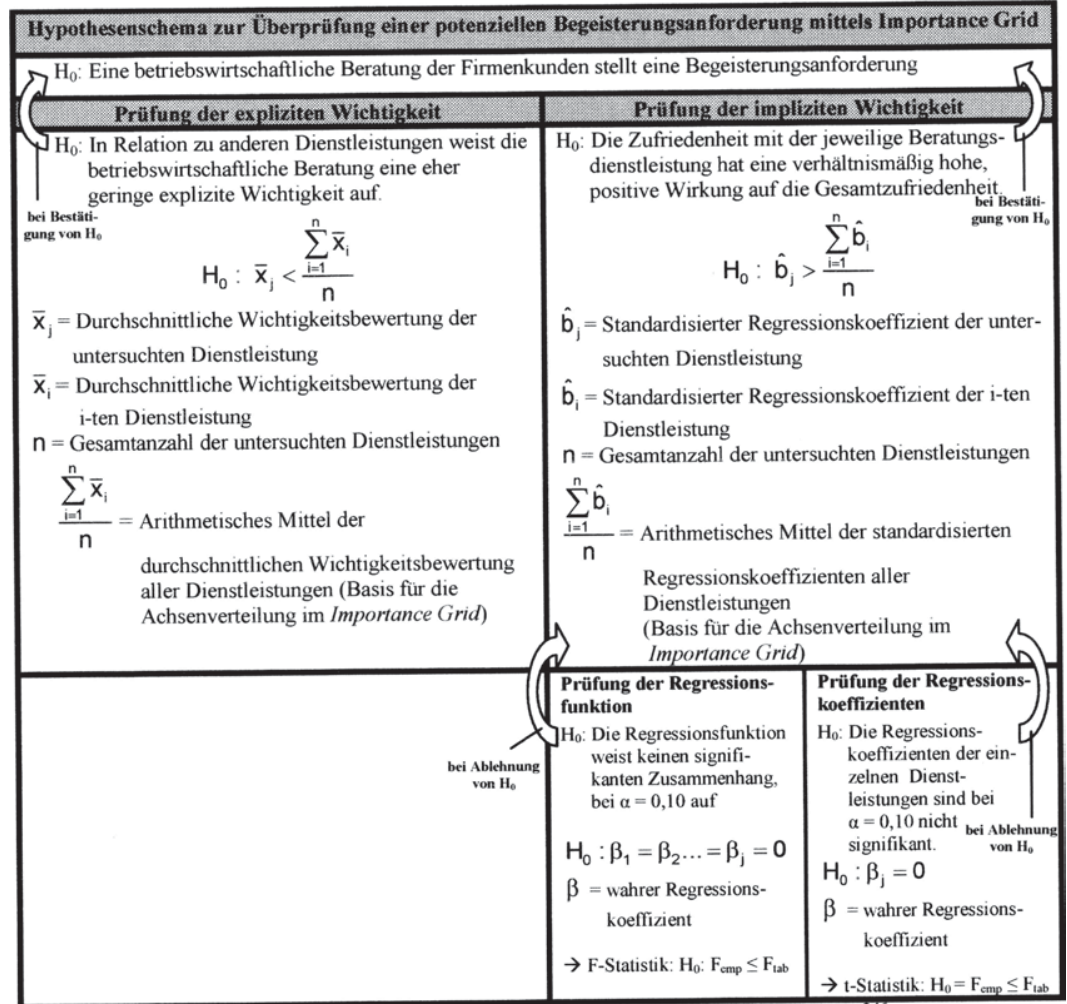

Abbildung 27: Hypothesenschema zur Analyse von Begeisterungsanforderungen ${ }^{2+}$

246 Eigene Darstellung. 
Aus Gründen der Übersichtlichkeit wurde lediglich eine Nullhypothese $H_{0}$ formuliert. ${ }^{247}$ Diese wird bei der Signifikanzprüfung von Regressionsmodellen in der Regel so formuliert, dass ihre Ablehnung einen signifikanten Zusammenhang bestätigt. ${ }^{248}$ Diesem Sachverhalt wurde in der Darstellung Rechnung getragen. Die Hypothesentests bauen aufeinander auf und folgen insofern einer hierarchischen Struktur (zu lesen von unten nach oben).

Die verallgemeinerten Hypothesenschemata der Anforderungsdimensionen finden sich in Anhang 14. Nach Klärung des Untersuchungszusammenhangs soll die Durchführung der Analyse und die sich daraus ableitenden Ergebnisse detailliert vorgestellt werden.

\subsubsection{Vorgehensdarstellung}

Um ein möglichst aussagefähiges Ergebnis zu erhalten, wurde die Anwendung des Importance Grid separat für die Attributtypen Dienstleistungen und Beziehungsmerkmale durchgeführt.

Es soll nun zunächst die Anforderungsstruktur hinsichtlich des Dienstleistungsportfolios behandelt werden. Gemäß des zuvor beschriebenen hierarchischen Ansatzes wurde zunächst eine multipel lineare Regression durchgeführt. ${ }^{249}$ Hierzu wurde die in Frage 1.6 erfragte Gesamtzufriedenheit mit der Hausbank als abhängige Variable deklariert und mit den in Frage 2.2 ermittelten Einzelzufriedenheiten regressiert.

In diesem Zusammenhang musste die Missing Value-Problematik in besonderem $\mathrm{Maße}$ berücksichtigt werden. Dies ist damit zu begründen, dass die Einschätzung der Zufriedenheit mit Bankleistungen eine vorherige Leistungsinanspruchnahme erfordert. $\mathrm{Da}$ aber auch die Untersuchung innovativer Beratungsleistungen Gegenstand der Befragung war, ließen sich fehlende Werte in der Erhebung nicht vermeiden. In der Ergebnisauswertung wurden verschiedene Optionen zum Umgang mit dem Problem geprüft:

Aufgrund des Stichprobenumfangs und des Analysekontexts kamen als Verfahren nur der Paarweise Fallausschluss und die Methode Ersatz durch den Mittelwert in Frage. ${ }^{250}$ Bei der erstgenannten Option wird die fehlende Variable aus der Grundgesamtheit eliminiert, die restlichen Variablen werden jedoch weiterhin berücksichtigt. Es erfolgte jeweils eine situative Verfahrensauswahl.

247 In statistische Testverfahren wird die Vermutung über die Größe des wahren Parameters in einer Nullhypothese $H_{0}$ festgehalten. Für den Fall das die Nullhypothese nicht verworfen werden muss kann, wird eine Gegenhypothese $H_{l}$ formuliert, die angibt was bei einer Ablehnung geschehen soll, vgl. Hippmann, Hans-Dieter (2003), S. 293 f..

248 Vgl. Backhaus, Klaus et al. (2006), S. $68 \mathrm{ff}$..

249 Die Analyse basiert auf einer elektronischen Datenbank, in der die schriftlich eingereichten Fragebögen manuell nachgepflegt wurden. Die Auswertung erfolgte mit der Statistiksoftware SPSS.

250 Vgl. hierzu detailliert Backhaus et al.(2006), S. $151 \mathrm{ff}$. 
Allerdings führte zunächst keines der Verfahren zu einem signifikanten Ergebnis. Eine Vielzahl von Dienstleistungen wurde SPSS-seitig direkt von der Analyse ausgeschlossen. Im Rahmen der Prüfung der Regressionsfunktionen war die Nullhypothese jeweils beizubehalten. Das korrigierte Bestimmtheitsmaß, dass angibt welcher Anteil der Streuung in der Stichprobe durch die Regressionsfunktion erklärt werden kann, wies dabei jeweils einen sehr niedrigen Wert von 0,3 auf. Der F-Test mit dem analysiert wird, ob die Gesamtheit der unabhängigen Variablen in kausalem Zusammenhang zur abhängigen Variablen steht, erwies sich ebenfalls als nicht signifikant. Um die Ergebnisursache zu ergründen wurden die Attribute untereinander auf Interdependenzen untersucht. Dazu wurden diese in einer Korrelationsmatrix gegenübergestellt und einer Kolinearitätsanalyse unterzogen. Es zeigte sich, dass die nicht interpretierbaren Ergebnisse durch die gegenseitige Abhängigkeit einiger erklärender Variablen bedingt waren. Um auszuschließen, dass dieser Effekt durch den paarweisen Fallausschluss hervorgerufen wurde, wurde zusätzlich eine Regression mit Dummy-Variablen durchgeführt, in der die Gesamtzufriedenheit als abhängige und die Inanspruchnahme der jeweiligen Dienstleistungen als unabhängige Variablen festgelegt wurden. Dies führte zu keiner Ergebnisverbesserung.

Daher wurden die Attribute mit Hilfe einer vorgeschalteten Faktorenanalyse zusammengefasst, um auf diese Weise der Multikolinearität zu begegnen. Als Extraktionsmethode wurde die Hauptkomponentenanalyse gewählt, da die Zielsetzung der vorliegenden Faktorenanalyse ist, gleichartige Variablen unter einem geeigneten Sammelbegriff zu subsumieren. Die Anzahl der Faktoren wurde anhand der zwei Kriterien Extraktion bis zur Erklärung von 95\% der Varianz und der Kaiser-Methode bestimmt. ${ }^{251}$

Eine weitgehend plausible Attributzuordnung konnte durch das Kaiser-Kriterium erreicht werden. Im Einzelnen ergibt sich für die Bankleistungen nach Faktorrotation folgende 9-Faktor-Struktur (vgl. Tabelle 8), die ca. 70\% der Varianz der Stichprobe zu erklären

vermag:

Die ermittelten Faktoren wurden sodann erneut in eine Regressionsanalyse eingebunden. Die Regressionsfunktion erwies sich als signifikant gegen $\alpha=0,10$. Die Überprüfung der Regressionskoeffizienten ergab für alle Variablen bis auf die Faktoren 7 und 9 einen positiven Einfluss auf die Gesamtzufriedenheit.

${ }^{251}$ Für eine Übersicht der Faktorenextraktionskriterien vgl. Backhaus, Klaus er al. (2006), S. 314 


\begin{tabular}{|c|c|c|}
\hline $\begin{array}{l}\text { Faktor 1: } \\
\text { Banknahe bwl. Beratungs- } \\
\text { leistungen }\end{array}$ & $\begin{array}{l}\text { Faktor 2: } \\
\text { Finanzierungsleistungen }\end{array}$ & $\begin{array}{l}\text { Faktor 3: } \\
\text { Anlageberatung }\end{array}$ \\
\hline $\begin{array}{l}\text { - Bilanzanalysegespräch } \\
\text { - Strategiegespräch } \\
\text { - Ratingberatung } \\
\text { - Liquiditätsplanung } \\
\text { - Stärken-Schwächen-Analyse } \\
\text { - Risikoanalyse } \\
\text { - Planbilanz } \\
\text { - Existenzgründungsberatung }\end{array}$ & $\begin{array}{l}\text { - Hypothekenvermittlung } \\
\text { - Investitionskredite } \\
\text { - Öffentliche Fördermittel } \\
\text { - Bürgschafts- und Garantiegeschäft } \\
\text { - Leasing }\end{array}$ & $\begin{array}{l}\text { - Vermögensberatung } \\
\text { - Private Vermögensplanung } \\
\text { - Anlagemanagement }\end{array}$ \\
\hline $\begin{array}{l}\text { Faktor 4: } \\
\text { Innovative Beratungsleistungen }\end{array}$ & $\begin{array}{l}\text { Faktor 5: } \\
\text { Betriebswirtschaftliches } \\
\text { Serviceangebot }\end{array}$ & $\begin{array}{l}\text { Faktor 6: } \\
\text { Versicherungen }\end{array}$ \\
\hline $\begin{array}{l}\text { - Mezzanine Finanzierung } \\
\text { - Projektbeurteilung } \\
\text { - Beratung von Auslandsprojek- } \\
\text { ten } \\
\text { - Forderungsmanagemnt } \\
\end{array}$ & $\begin{array}{l}\text { - Investitionsplanung } \\
\text { - Workshops } \\
\text { - Unternehmensnachfolgeberatung }\end{array}$ & $\begin{array}{l}\text { - Lebensversicherungen } \\
\text { - Sachversicherungen } \\
\text { - Betriebliche Altersvorsorge }\end{array}$ \\
\hline $\begin{array}{l}\text { Faktor 7: } \\
\text { kein geeigneter Oberbegriff }\end{array}$ & $\begin{array}{l}\text { Faktor 8: } \\
\text { Liquiditätsmanagement }\end{array}$ & $\begin{array}{l}\text { Faktor 9: } \\
\text { AuBenhandelsleistungen }\end{array}$ \\
\hline $\begin{array}{l}\text { - Immobilienvermittlung } \\
\text { - Zins- und Währungsmanage- } \\
\text { ment }\end{array}$ & $\begin{array}{l}\text { - Cash Management } \\
\text { - Betriebsmittelkredite } \\
\text { - Elektronischer Zahlungsverkehr }\end{array}$ & $\begin{array}{l}\text { - Auslandszahlungsverkehr } \\
\text { - Im- und Exportfinanzierung }\end{array}$ \\
\hline
\end{tabular}

Tabelle 8: Faktorenzuordnung Dienstleistungen (geordnet nach Faktorladungen)

Für die horizontale Achse des Importance Grid waren nun noch die ebenfalls in Frage 2.2 ermittelten expliziten Wichtigkeiten auszuwerten. Da diese auf Dienstleistungsebene ermittelt wurden, galt es die arithmetischen Mittelwerte der Einzelattribute anhand ihrer relativen Faktorladung zu einer Wichtigkeitseinstufung auf Faktorebene zusammenzufassen. Das Importance Grid der Dienstleistungsfaktoren sieht demnach wie folgt aus:

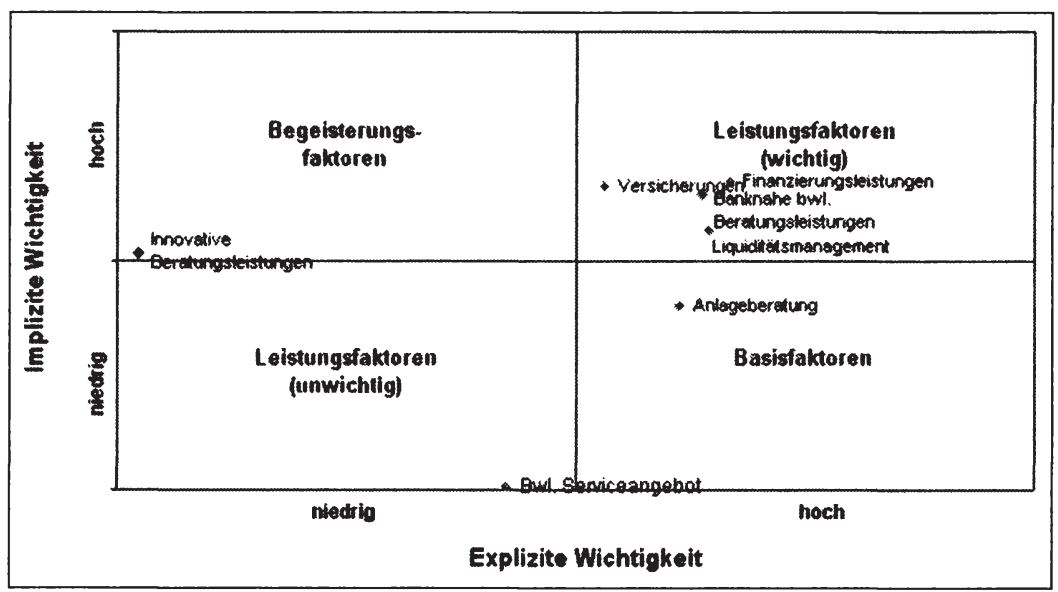

Abbildung 28: Importance Grid: Dienstleistungsfaktoren 
Da es sinnvoll erscheint auch die anderen untersuchten Sachverhalte in eine Ergebnisinterpretation mit einzubeziehen, soll zunächst die Untersuchung der Beziehungsmerkmale dargestellt werden.

Analog zur Vorgehensweise bei den Dienstleistungsattributen wurde zunächst eine multiple Regression der in Frage 3.1 aufgeführten Einzelmerkmale durchgeführt. Während sich die Regressionsfunktion dabei als signifikant erwies, ergab die Prüfung der Regressionskoeffizienten kein aussagefähiges Ergebnis. Wie zuvor auch, lag der Grund dafür in der verhältnismäßig starken Korrelation der abhängigen Variablen. Somit wurde erneut eine Faktorenanalyse durchgeführt, um die Interdependenzen durch Zusammenfassung zu eliminieren. Auffallend war dabei, dass das Merkmal Private Kontakte in der Korrelationsmatrix nur sehr geringe Korrelationswerte aufwies. Es ist damit für faktoranalytische Zwecke ungeeignet und war damit aus der Untersuchung auszuschließen. Der Kaiser-Meyer-Olkin-Test bescheinigt den übrigen Ausgangsvariablen mit dem Status marvelous eine besonders gute Eignung für eine Faktorenanalyse. ${ }^{252}$ Aufgrund dessen konnte die Faktorenextraktion auch anhand des Kriteriums Extraktion bis zur Erklärung von 95\% der Varianz erfolgen. Danach ergeben sich die folgenden 14 Attributgruppen:

\begin{tabular}{|c|c|c|}
\hline $\begin{array}{l}\text { Faktor 1: } \\
\text { Betreueraktivität }\end{array}$ & $\begin{array}{l}\text { Faktor 2: } \\
\text { Verlässlichkeit }\end{array}$ & $\begin{array}{l}\text { Faktor 3: } \\
\text { Vertrautheit }\end{array}$ \\
\hline $\begin{array}{l}\text { - Ansprache betriebswirt- } \\
\text { schaftlicher Handlungsfelder } \\
\text { - Gegenüberstellung von } \\
\text { Alternativen } \\
\text { - Ansprache Optimierungs- } \\
\text { potenzial Finanzdienstleistungen }\end{array}$ & $\begin{array}{l}\text { - Termintreue } \\
\text { - Zuverlässigkeit }\end{array}$ & $\begin{array}{l}\text { - Persönlicher Ansprechpartner } \\
\text { - Regelmäßiger persönlicher } \\
\text { Kontakt } \\
\text { - Ähnliche Wertvorstellungen }\end{array}$ \\
\hline $\begin{array}{l}\text { Faktor 4: } \\
\text { Angenehmes Umfeld }\end{array}$ & $\begin{array}{l}\text { Faktor } 5: \\
\text { Branchenbezug }\end{array}$ & $\begin{array}{l}\text { Faktor 7: } \\
\text { Nachvollziehbarkeit }\end{array}$ \\
\hline $\begin{array}{l}\text { - Diskrete Beratungsräume } \\
\text { - Angenehme Atmosphäre }\end{array}$ & $\begin{array}{l}\text { - Regelmäßige Brancheninforma- } \\
\text { tionen } \\
\text { - Branchenkenntnis } \\
\end{array}$ & $\begin{array}{l}\text { - Transparenz } \\
\text { - Fairness }\end{array}$ \\
\hline $\begin{array}{l}\text { Faktor 7: } \\
\text { Offenheit }\end{array}$ & $\begin{array}{l}\text { Faktor } 8 \text { : } \\
\text { Externe Beratungstermine }\end{array}$ & $\begin{array}{l}\text { Faktor 9: } \\
\text { Hinzuziehung von Spezialisten }\end{array}$ \\
\hline - Offenheit & - Externe Beratungstermine & $\begin{array}{l}\text { - Auslandszahlungsverkehr } \\
\text { - Im- und Exportfinanzierung }\end{array}$ \\
\hline $\begin{array}{l}\text { Faktor 10: } \\
\text { Schnelle Entscheidungen }\end{array}$ & $\begin{array}{l}\text { Faktor 11: } \\
\text { Konstanz der Bezugsperson }\end{array}$ & $\begin{array}{l}\text { Faktor 12: } \\
\text { Individuelle Betreuung }\end{array}$ \\
\hline - Schnelle Entscheidungen & - Konstanz der Bezugsperson & - Individuelle Betreuung \\
\hline $\begin{array}{l}\text { Faktor 13: } \\
\text { Kompetenz }\end{array}$ & $\begin{array}{l}\text { Faktor 14: } \\
\text { Vertrauensbereitschaft }\end{array}$ & \\
\hline $\begin{array}{l}\text { - Betriebswirtschaftliche } \\
\text { Kompetenz } \\
\text { - Bankfachliche Kompetenz }\end{array}$ & $\begin{array}{l}\text { - Gegenseitige Vertrauensbereit- } \\
\text { schaft }\end{array}$ & \\
\hline
\end{tabular}

Tabelle 9: Faktorenzuordnung Beziehungsattribute (geordnet nach Faktorladungen)

${ }^{252} \mathrm{Vgl}$. Backhaus, Klaus et al. (2006), S. $276 \mathrm{f}$. 
Wie aus Tabelle 9 erkennbar ist, war die Faktorladung bei einigen Merkmalen so eindeutig, dass sie keiner Zusammenfassung bedurften. Attribute mit ähnlichen Ladungswerten bei mehr als einem Faktor wurden sachlogisch zugeordnet.

Die Überprüfung der Regressionsfunktion der nachgelagerten Regressionsanalyse auf Faktorebene ergab keine Beanstandungen. Allerdings konnte den Faktoren Branchenbezug, Nachvollziehbarkeit, Offenheit, Externe Beratungstermine und Individuelle Betreuung im Rahmen des Regressionsmodells kein Einfluss auf die Gesamtzufriedenheit nachgewiesen werden. Die übrigen Regressoren erwiesen sich als signifikant gegen $\alpha=0,05$. Die Aggregation der erhobenen Einzelwichtigkeiten zu einer Faktorbewertung, erfolgte wie zuvor beschrieben, gewichtet. Somit ergab sich für die Beziehungsfaktoren das nachfolgende Importance Grid:

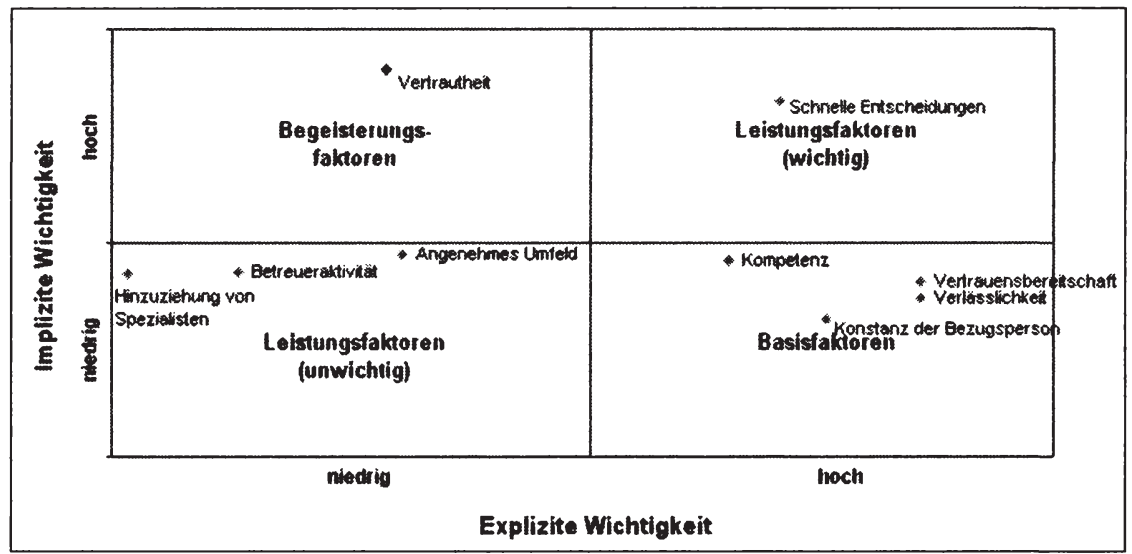

Abbildung 29: Importance Grid: Beziehungsfaktoren

\subsection{Ergebnisinterpretation unter Einbezug der Nebenuntersuchungen}

Die ermittelte Anforderungsstruktur soll nachfolgend je Attributtyp und unter Berücksichtigung der Ergebnisse der Nebenuntersuchungen interpretiert werden.

\subsubsection{Interpretation der Dienstleistungsfaktoren}

Auf Grundlage der Ergebnisse der Faktorenanalyse war bereits zu vermuten, dass die Erwartungshaltung an betriebswirtschaftliche Dienstleistungen differiert. Bemerkenswert ist, dass das Angebot von banknahen Beratungsleistungen keine Begeisterungswirkung mehr entfalten kann, sondern mittlerweile in der Anforderungshaltung fest verankert zu sein scheint. Dies hängt wahrscheinlich u.a. mit der Entwicklung einer Ratingkultur in der Kunde-Bank-Beziehung zusammen. Im Rahmen der turnus- 
gemäßen Rating-Erstellung hat sich ein betriebswirtschaftlicher Austausch etabliert, der bankseitig vielfach noch nicht optimal genutzt wird. ${ }^{253}$

Ein höheres Begeisterungspotenzial haben hingegen innovative Beratungsleistungen mit Finanzwirtschaftsbezug, sowie Beratungen mit Projektcharakter. Dieses Ergebnis bestätigt die Forderung der Firmenkunden nach passgenauen und bedarfsspezifischen Lösungen. Geht man in der Interpretation noch etwas weiter, kann in der Erwartung einer projektbezogenen Betreuung auch der Wunsch nach einer intensiveren „Teamarbeit“ gesehen werden. Des Weiteren lässt sich ein Bezug zur wachsenden Bedeutung von Auslandsaktivitäten im Mittelstand herstellen. ${ }^{254}$

Interessant ist auch, dass ein Teil des betriebswirtschaftlichen Leistungsangebotes von untergeordnetem Interesse ist. Bei der Unternehmensnachfolgeberatung und den Workshops mag das daran liegen, dass diese Leistungen nicht die Kernkompetenz von Banken wahrgenommen werden wird. Hinsichtlich der Investitionsplanung konnte jedoch durch eine Korrelationsanalyse gezeigt werden, dass das wünschenswerte Engagement in diesem Bereich positiv mit der Kompetenzvermutung korreliert. ${ }^{255}$

Die Positionierung des Faktors Anlagemanagement als Basisanforderung kann so interpretiert werden, dass der Anlagebereich im mittelständischen Firmenkundengeschäft einen relativ hohen Standardisierungsgrad aufweist und aufgrund der eingeschränkten Differenzierungsmöglichkeiten nur eine verhältnismäßig geringe Zufriedenheits- und Emotionswirkung zur Folge hat. Ein weiterer Effekt ist in der geringen Leistungsinanspruchnahme der privaten Vermögensplanung zu sehen, die über ein breiteres Differenzierungspotenzial verfügt.

\subsubsection{Interpretation der Beziehungsfaktoren}

Bei der Verteilung der Beziehungsfaktoren im Importance Grid fällt zunächst die deutliche Positionierung des Faktors Vertrautheit im Bereich der Begeisterungsanforderungen auf. Da die Vertrautheit - wie in Kapitel 4.2.3 beschrieben - eine entscheidende Vorlaufvariable der Vertrauensentstehung und somit eine wichtige Einflussgröße der Kundenbindung darstellt, sollte dieses Ergebnis in der Betreuungsstrategie gesondert berücksichtigt werden. Die theoretische Annahme, dass es sich bei der Dimension der Vertrautheit um eine Begeisterungsanforderung handelt, kann also durch die Empirie bestätigt werden.

Die von SEGBERS auch im Bereich der Begeisterungsanforderungen angesiedelte Konstanz der Bezugsperson wird von den Firmenkunden jedoch als selbstverständlich vorausgesetzt. Dieser Anspruch kann jedoch aufgrund von Fluktuationsbewegungen nicht immer erfüllt werden.

\footnotetext{
${ }^{253}$ Vgl. hierzu auch Kapitel 9

254 Vgl. Kapitel 3.1.3

255 Vgl. Tabelle 19 in Anhang 14
} 
Die Forderung nach effizienten Prozessen und damit indirekt auch nach einer effizienten Ressourcensteuerung wird im Importance Grid durch die Positionierung des Faktors Schnelle Entscheidungen deutlich.

Die restlichen Faktoren haben eine mittlere, implizite Wichtigkeit gemeinsam, was strukturell tendenziell zu einer Verschiebung innerhalb der Quadranten führen kann. Daher sollten insbesondere die Faktoren Hinzuziehung von Spezialisten, Betreueraktivität und Angenehme Atmosphäre nicht vernachlässigt werden, da in ihnen möglicherweise zukünftige Begeisterungsanforderungen zu vermuten sind.

Im Kontext der Beziehungsmerkmale sollen abschließend die Ergebnisse der Regressionsanalyse zu den in Frage 3.2 erhobenen Bindungswirkungen aufgenommen werden. Dazu wurde die Stärke der Bindung an die Hausbank als abhängige und die Bindungsdimensionen Ge- und Verbundenheit, sowie die Bindungswirkung des Dienstleistungsportfolios als unabhängig Variablen deklariert. Das Regressionsmodell erwies sich als signifikant. Hinsichtlich der Regressoren konnte kein direkter Kausalzusammenhang zwischen dem Leistungsangebot und der Bindungsstärke nachgewiesen werden. Somit ist die These zu bestätigen, dass ein Bindungszustand immer einer personellen Interaktion bedarf. Die Regressionsanalyse attestiert des Weiteren der Gebundenheit eine stärkere Bindungswirkung als der Verbundenheit. Dieses Ergebnis ist jedoch kritisch zu prüfen, da ein Zustand der Verbundenheit ein eher emotiv basiertes Phänomen darstellt und deshalb in der rationalen Wahrnehmung nicht unbedingt präsent ist. Zur Validierung des Sachverhaltes könnten in einer Befragung gezielt Determinanten der Bindungsdimensionen eingesetzt werden, um rationale Bewertungsmuster durch eine differenziertere Erhebung zu relativieren. 


\section{Optimierung des Leistungsportfolios}

Im Abschlusskapitel dieser Arbeit sollen aus dem entwickelten Portfolio-Ansatz zur potenzialorientierten Kundensegmentierung und den Erkenntnissen der Anforderungsanalyse einige Handlungsempfehlungen zur Ableitung einer segmentspezifischen Betreuungsstrategie erarbeitet werden. Dazu sollen in einem groben Überblick Implikationen für die segmentspezifische Gestaltung des Leistungsportfolios und die Steuerung der Vertriebsressourcen vorgestellt werden.

\subsection{Segmentspezifische Festlegung des Leistungsangebotes}

Die ganzheitliche Betreuungsstrategie intendiert einen kundenindividuellen Zuschnitt der Leistungspalette auf die jeweilige Kundensituation. Allerdings muss durch das Vertriebsmanagement gewährleistet werden, dass eine angemessene Rentabilität je Feinsegment erreicht wird. Ein Steuerungsmodell ist dabei in der segmentspezifischen Festlegung des Leistungsportfolios zu sehen. Die Auswahl der Produkte und Dienstleistungen sollte sich dabei Ertrags-, Potenzial- und Bedarfslage, sowie der Grobsegmentierung orientieren. Daneben besteht zusätzlich die Möglichkeit der segmentorientierten Preisdifferenzierung.

Von besonderem Interesse sind auch Mehrwertdienstleistungen, die als Zusatzservices angeboten werden, um eine gezielte Kundenbegeisterung zu erreichen. Für die Feinsegmente können exemplarisch folgende Leistungsstrategien unterschieden werden:

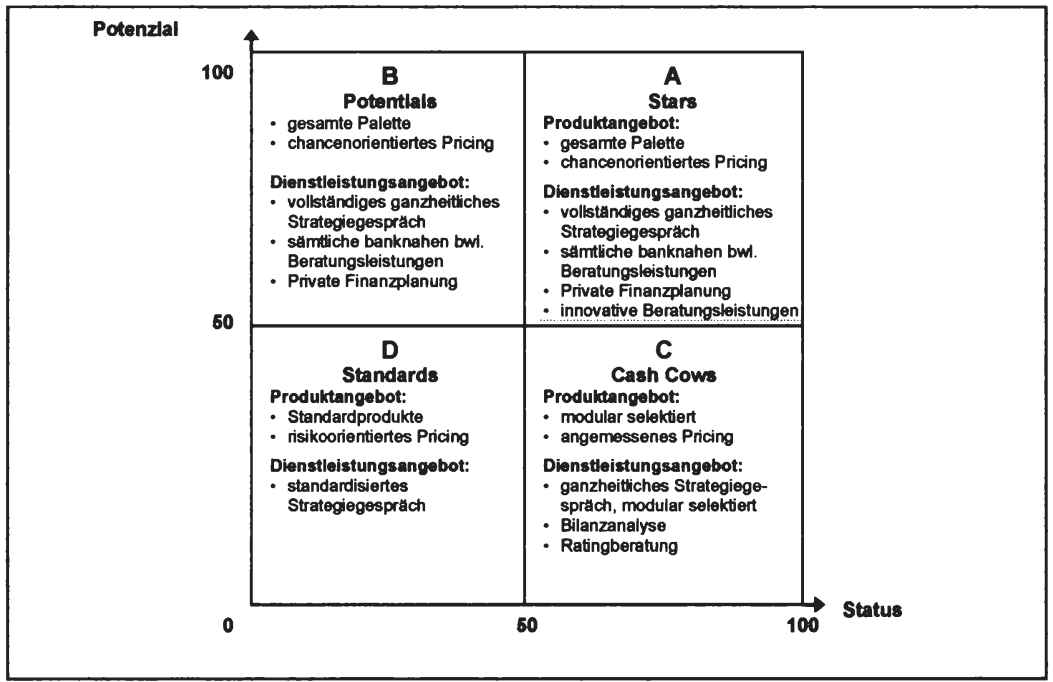

Abbildung 30: Firmenkunden-Portfolio: Leistungsstrategien 
Die Analyse der Anforderungsstruktur hat ergeben, dass das betriebswirtschaftliche Beratungsangebot eine wichtige Leistungsanforderung darstellt. Hier gilt es den ganzheitlichen Betreuungsansatz konsequent zu verfolgen und sich durch die systematische Vorgehensweise vom Wettbewerb abzugrenzen. Dazu stehen im Genossenschaftssektor eine Reihe strukturierter Beratungshilfen zur Verfügung, die den partnerschaftlichen Dialog fördern. ${ }^{256}$

Die Festlegung des Produktportfolios kann dabei auch bei der Vertriebssteuerung von Interesse sein. So kann durch die Hinterlegung eines Soll-Produktportfolios, das noch nicht ausgeschöpfte Kundenpotenzial ermittelt, im Rahmen des Segmentierungslaufs festgehalten und als Vetriebsimpuls verwendet werden.

\subsection{Gezielte Ressourcensteuerung je Segment}

Aufgrund der begrenzten Betreuungsressourcen bedarf es - wie bereits in Kapitel 5 ausgeführt - einer Priorisierung der Betreuungsaktivitäten, um eine effiziente Marktbearbeitung zu ermöglichen.

Aus der Feinsegmentierung lassen sich folgende Ansätze zur Ressourcensteuerung entwickeln:

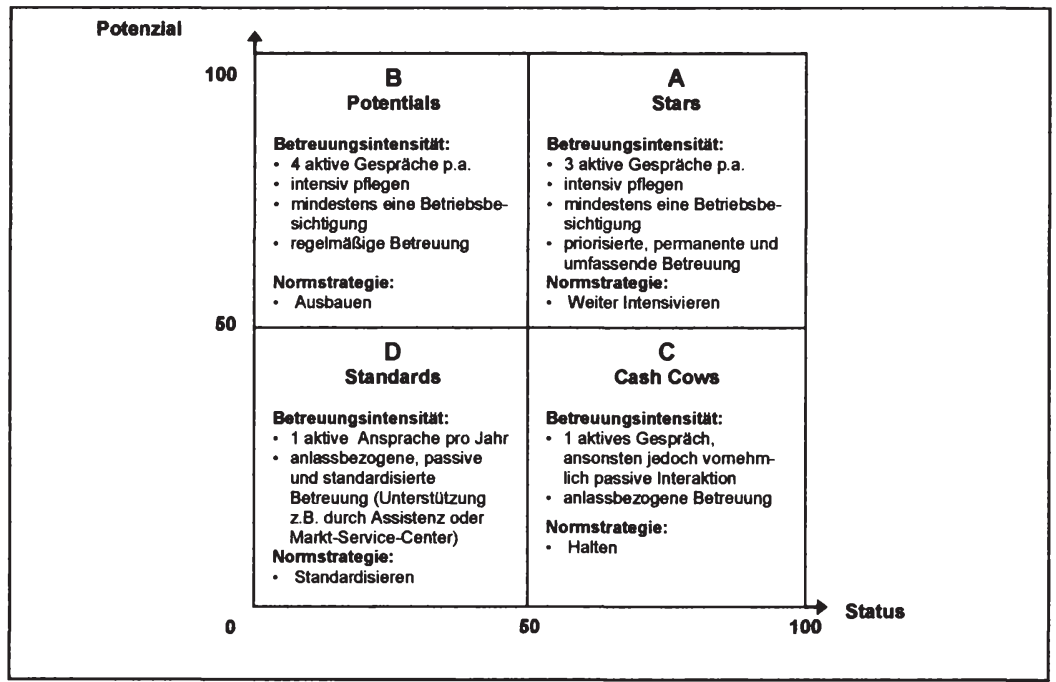

Abbildung 31: Firmenkundenportfolio: Ressourcensteuerung

${ }^{256}$ z.B. die Software MinD.banker 
Die dargestellten segmentspezifischen Normstrategien stehen dem Firmenkundenbetreuer als Leitlinie für den Einsatz der Betreuungsressourcen vor. Bei Bedarf kann - beispielsweise nach Scorewerten kategorisiert - eine noch feinere Ressourcensteuerung erreicht werden. Durch das jährliche Strategiegespräch und das damit verbundene Segmentierungsprozedere ergibt sich ein Regelkreislauf der Kundenbetreuung, der einen wichtigen Wettbewerbsvorteil darstellen kann.

Für die Volksbanken und Raiffeisenbanken ist abschließend zu erwähnen, dass der hier entwickelte Feinsegmentierungsansatz zukünftig als Grundlage eines potenzialorientierten Vertriebsmanagements genutzt werden kann, da die konzeptionellen Überlegungen dieser Arbeit in ein Softwareprojekt für das genossenschaftliche Firmenkundengeschäft eingehen. Das Verfahren bietet einen Modellrahmen der auch zur Leistungs- und Preisdifferenzierung herangezogen werden kann und eine primäre Konzentration der Ressourcen auf interessante Geschäftsbeziehungen ermöglicht. Trotz der beschrieben Fokussierung wird durch die Kopplung an den ganzheitlichen Betreuungsansatz eine bedarfsorientierte Betreuung aller Kundengruppen möglich. 


\section{Fazit}

In der vorliegenden Arbeit wurde ein umfassender Ansatz zur Integration einer potenzialorientierten Kundensegmentierung in ein ganzheitliches Vertriebskonzept entwickelt.

Neuartig an der Vorgehensweise ist, dass dieser direkt an einen Beratungsansatz gekoppelt wurde und sämtliche Wechselwirkungen entlang des Vertriebsprozesses Berücksichtigung fanden. Somit wird dem Aspekt der Kundenorientierung Rechnung getragen, ohne Rentabilitäts- und Effizienzanforderungen im Firmenkundengeschäft außer Acht zu lassen. Insofern kann von einer „efficient customization “257 gesprochen werden, die als zentrales Instrument eines erfolgreichen Kundenbindungsmanagements fungiert. Durch Verknüpfung der Erkenntnisse der empirischen Erhebung mit dem entwickelten Segmentierungsansatz kann eine Leistungs- und Betreuungsdifferenzierung erreicht werden, indem feinsegmentspezifische Normstrategien abgeleitet werden.

Außerdem konnte durch die Analyse der Kundenanforderungen, die Wichtigkeit banknaher Beratungsdienstleistungen nachgewiesen werden. Hinsichtlich des Rollenverständnisses des Firmenkundenbetreuers wurde gezeigt, dass bei finanznahen Themen die Rolle des unternehmerischen Ratgebers erwartet wird. Betreuerseitig sollte dies als Differenzierungschance verstanden werden, die etwa durch leichte Modifikation (z.B. durch qualitätssteigernde Zusatzkomponenten) der erwarteten Beratungsleistungen genutzt werden kann. Insofern wird es interessant sein, zu beobachten, wie sich die bankbetriebswirtschaftlichen Beratungsprozesse verändern werden.

Durch eine regelmäßige Analyse der Faktorstruktur der Kundenanforderungen, können sich abzeichnende Trends schnell erkannt und antizipiert werden. In diesem Zusammenhang wäre es interessant, die Auswertung nicht nur auf Basis von Befragungen, sondern auch aus Gesprächsinformationen (z.B. Strategiedialog, etc.) zu speisen.

Insgesamt wird mit Spannung erwartet, wie der entwickelte Ansatz - nach softwaretechnischer Umsetzung in einer Multi-Banken-Version - in die Vertriebspraxis aufgenommen wird, ob die Validität der Segmentierungsergebnisse empirisch nachgewiesen werden kann und inwiefern die hier geforderte ganzheitliche Integration der Methodik in der Bankpraxis gelingt.

257 In Anlehnung an den in der Konsumgüterindustrie häufig verwendeten Begriff der Mass Customization. Während Massenprodukte beispielsweise in unterschiedlichen Farbkombinationen angeboten werden, um beim Verbraucher ein Gefühl von Individualität zu erzeugen, soll der Fokus der Bankbetreuung weiterhin auf der individuellen Beratung liegen, die nur standardisiert wird, wenn dies unter Rentabilitäts- und Effizienzgesichtspunkten erforderlich ist. 


\section{Anhang}

\section{Anhangverzeichnis}

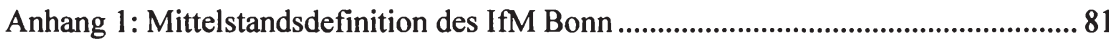

Anhang 2: Firmenkunden einer Genossenschaftsbank nach Sektoren ........................... 81

Anhang 3: Wettbewerbsstrategien nach PORTER ........................................................ 82

Anhang 4: Anforderungsdimensionen in Hausbankbeziehungen.................................... 83

Anhang 5: Rating-Analyse in MinD.banker .................................................................. 84

Anhang 6: Segmentierungsansatz Roland Berger ........................................................ 85

Anhang 7: Kundensegmentierung - Kriterientypologisierung...................................... 87

Anhang 8: Portfolio-Methode der Boston Consulting Group.......................................... 88

Anhang 9: Portfolio-Methode von McKinsey ................................................................. 89

Anhang 10: Portfolio-Methode nach SCHRÖDER........................................................... 92

Anhang 11: Segmentierungskriterien zur Potenzialerhebung ......................................... 93

Anhang 12: Eigenentwickeltes Segmentierungstool ..................................................94

Anhang 13: Fragebogen und Anschreiben...................................................................... 98

Anhang 14: Auswertung der empirischen Erhebung................................................... 108 


\section{Anhang 1: Mittelstandsdefinition des IfM Bonn}

Mittelstandsdefinition des IfM Bonn seit Einführung des Euro (01.01.2002):

\begin{tabular}{|c|c|c|}
\hline Unternehmensgröße & Zahl der Beschäftigten & Umsatz $\boldsymbol{€}$ / Jahr \\
\hline klein & bis 9 & bis unter 1 Million \\
\hline mittel & 10 bis 499 & 1 bis 50 Millionen \\
\hline groß & 500 und mehr & 50 Millionen und mehr \\
\hline
\end{tabular}

Tabelle 10: Quantitative Mittelstandsdefinition des IfM Bonn

Quelle: IfM Bonn (2006)

\section{Anhang 2: Firmenkunden einer Genossenschaftsbank nach Sektoren}

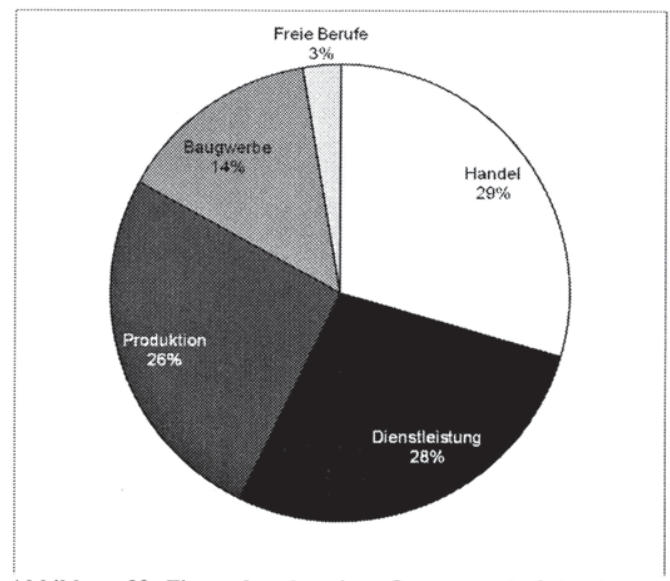

Abbildung 32: Firmenkunden einer Genossenschaftsbank nach Sektoren

Quelle: Eigene empirische Erhebung 


\section{Anhang 3: Wettbewerbsstrategien nach PORTER}

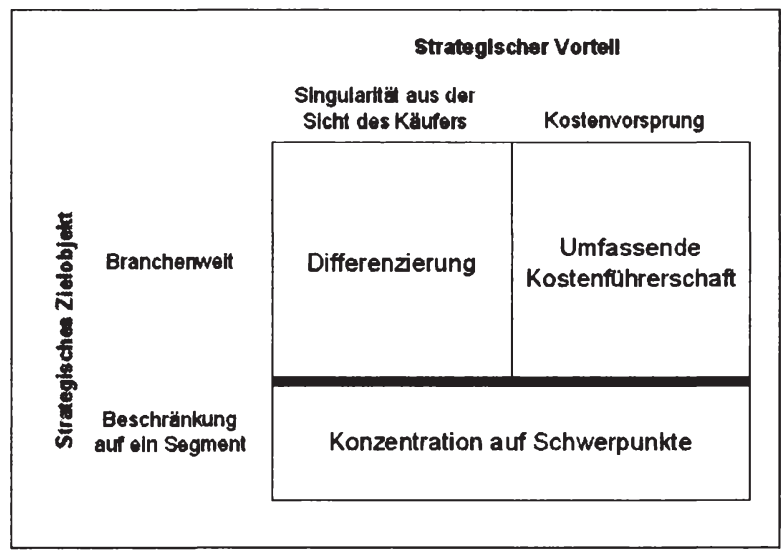

Abbildung 33: Wettbewerbsstrategien nach PORTER

Quelle: Porter, Michael E. (1999) 


\section{Anhang 4: Anforderungsdimensionen in Hausbankbeziehungen}

\begin{tabular}{|c|c|}
\hline $\begin{array}{l}\text { Qualitat } \\
\text { Dimension }\end{array}$ & Faktoren \\
\hline \multirow[t]{4}{*}{ Basisanforderungen } & Verhalten: Freundlichkeit; angenehme Atmosph"rre: Erreichbarkeit \\
\hline & Kompetenz: Fundierte Ausbildung; gute Produkt- und Organisationskenntnis \\
\hline & Dienstleistungsspektrum: umfangreiche und problembezogene Produktpalette \\
\hline & $\begin{array}{l}\text { tangibles Umfeld: Gepflegte Kleidung/Geschäftsräume; ad̋quate technische } \\
\text { Ausstattung }\end{array}$ \\
\hline \multirow[t]{4}{*}{ Leistungsanforderungen } & $\begin{array}{l}\text { Kontakt: RegelmäBiger, persönlicher Kontakt; Bequemlichkeit (Besuch in } \\
\text { Geschäftsräumen; Betriebsbesichtigung) }\end{array}$ \\
\hline & $\begin{array}{l}\text { Proaktivitat: Erkennen und Ansprechen von finanzierungstechnischen } \\
\text { Problemen; Hinzuziehung von Spezialisten }\end{array}$ \\
\hline & Abläufe: Unkomplizierte, zuverlässioe und einfache Prozesse \\
\hline & $\begin{array}{l}\text { Ergebnis: Kundenindividuelle, bedarfsgerechte (Finanzierungs-) Lösungen; } \\
\text { Analyse von Altemativen }\end{array}$ \\
\hline \multirow[t]{6}{*}{ Begeisterungsanforderungen } & $\begin{array}{l}\text { Persönliche Vertrautheit: Ähnlichkeit der Interaktionspartner; Soziale } \\
\text { Eingebundenheit der Interaktionspantner }\end{array}$ \\
\hline & $\begin{array}{l}\text { Kontakt: Konstanz der Bezugsperson (z.B. Dauer der persönlich-dyadischen } \\
\text { Beziehung) }\end{array}$ \\
\hline & $\begin{array}{l}\text { Außergewöhnliches Engagement: Proaktives Erkennen und Ansprechen von } \\
\text { unternehmerischen Problemen (FKB ais unternehmerischer Ratgeber); hohe } \\
\text { Branchenkenntnis; (erfolgreicher) Versuch, sich über organisationsinterne } \\
\text { Richtlinien hinwegzusetzen }\end{array}$ \\
\hline & $\begin{array}{l}\text { Schaffung von Mehrwert: Vermittlung von Kunden/Lieferanten/lmmobilien; } \\
\text { Angebot außergewöhnlicher Serviceleistungen }\end{array}$ \\
\hline & $\begin{array}{l}\text { Offenheit: Frühzeitige Ansprache von Problemen; Einräumung von } \\
\text { Schwächen (z.B. in Bezug auf eigene Produkte); ggifs. Integration von } \\
\text { Produkten fremder Anbieter (z.B. Förderkredite, kurzfristiger Verzicht auf } \\
\text { eigene Vorteile); Offener Ratingdialog; ähnliche Werte und Ziele }\end{array}$ \\
\hline & $\begin{array}{l}\text { Antizyklisches Verhalten. Fairness: Kein formales Vorgehen in } \\
\text { Krisensituationen (positive Behandlung auch bei (kurzfristig) schlechten } \\
\text { Zahlen; Verzicht auf umfangreiche Besicherung bei Existenzgründern) }\end{array}$ \\
\hline
\end{tabular}

Abbildung 34: Anforderungsdimensionen in Hausbankbeziehungen

Quelle: Segbers, Klaus (2007), S.313 


\section{Anhang 5: Rating-Analyse in MinD.banker}

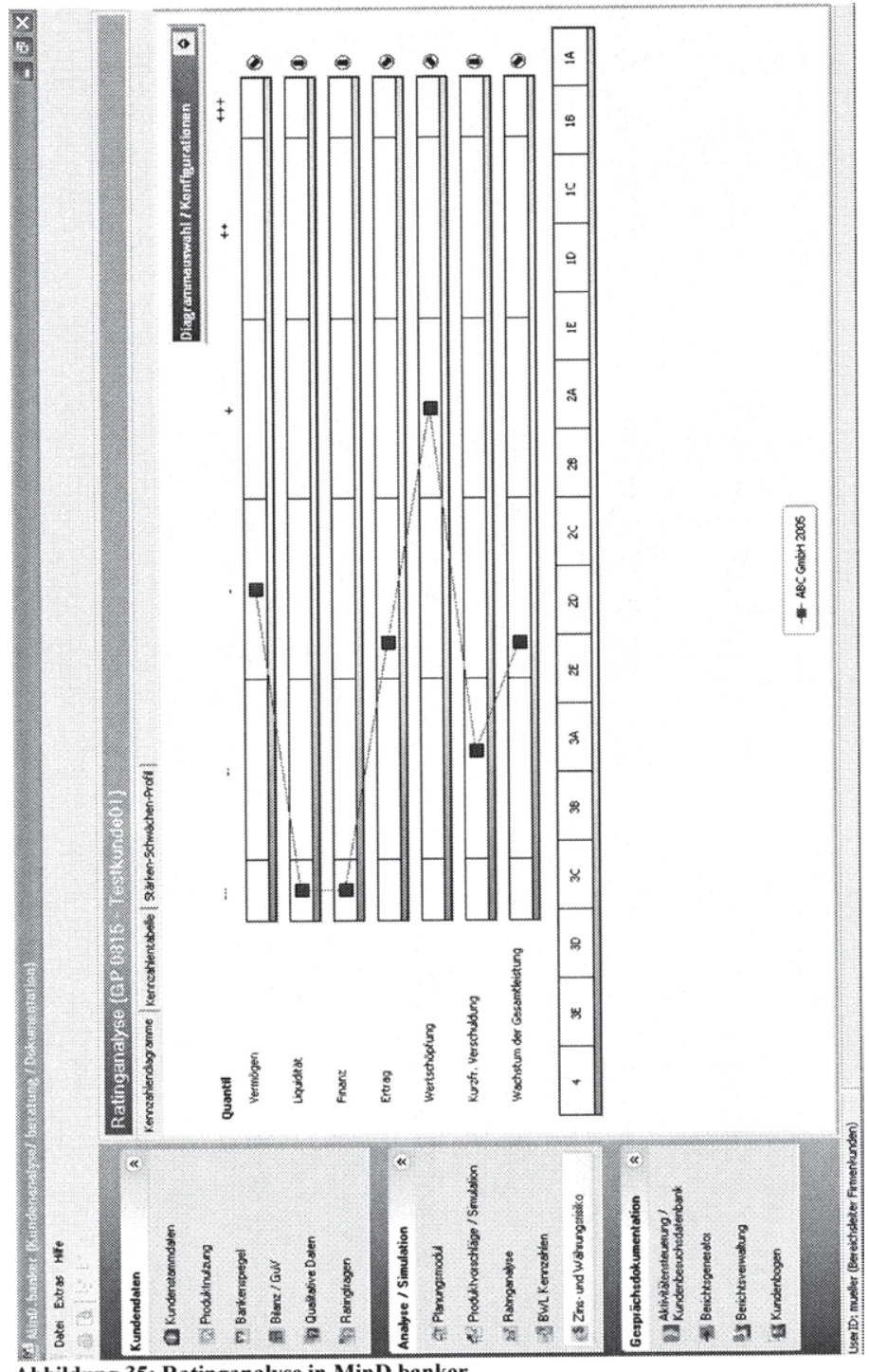

Abbildung 35: Ratinganalyse in MinD.banker

Quelle: BMS Consulting GmbH 


\section{Anhang 6: Segmentierungsansatz Roland Berger}

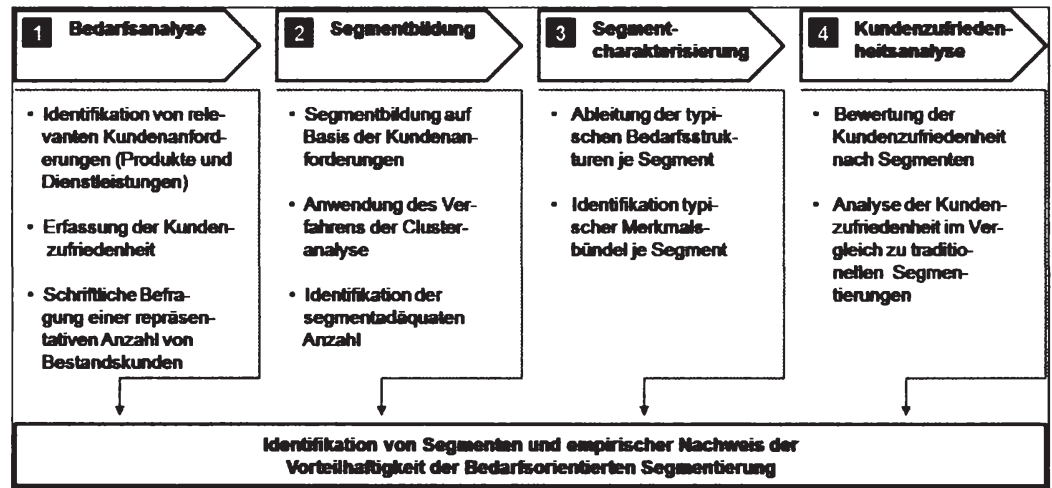

Abbildung 36: Analyseschritte und inhaltliche Vorgehensweise

Quelle: Bufka, Jürgen / Eichelmann, Thomas (2002), S. 130

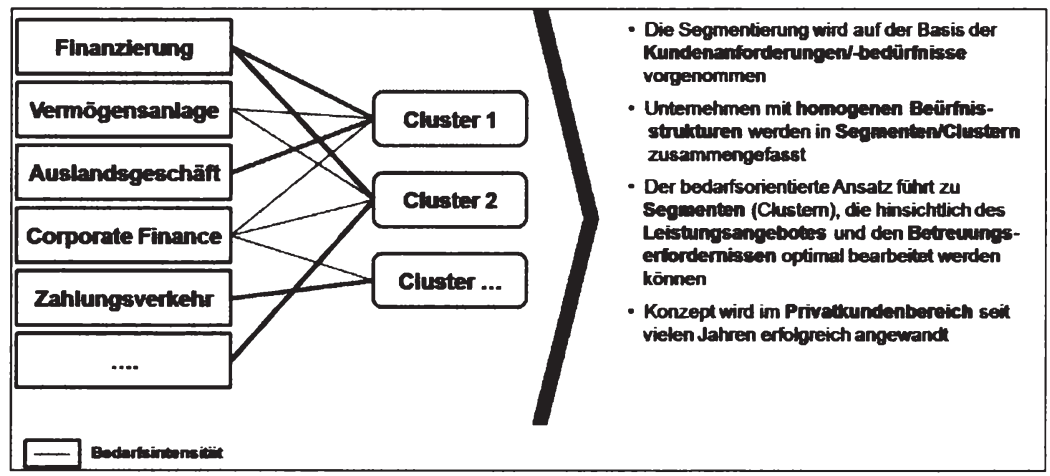

Abbildung 37: Cluster-Bildung

Quelle: Bufka, Jürgen / Eichelmann, Thomas (2002), S. 128 


\begin{tabular}{|c|c|c|c|c|c|}
\hline & Clustier 1 & Chuster 3 & Chaster 3 & Chester 4 & Chuster 5 \\
\hline $\begin{array}{l}\text { Hoher } \\
\text { Bedert }\end{array}$ & $\begin{array}{l}\text { - Zahlungsvertehr } \\
\text { - Kredigaschaft } \\
\text { - Vermbgansenlago }\end{array}$ & 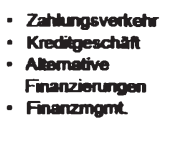 & $\begin{array}{l}\text { - Zahlungsverkehr } \\
\text { - Kredidgeschat } \\
\text { - Alternative } \\
\text { Finareienungen } \\
\text { - Fineromgint } \\
\text { - Auslandgeschafre }\end{array}$ & $\begin{array}{l}\text { - Zahlungsvertehr } \\
\text { - Vermbgensentage } \\
\text { - Alomative } \\
\text { Finanzierungen }\end{array}$ & 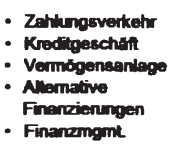 \\
\hline Fon- & $\begin{array}{l}\text { - Alemetive } \\
\text { Finanzierungen }\end{array}$ & $\begin{array}{l}\text { - Austandiseschene } \\
\text { - Corporate Finence }\end{array}$ & - Corporrete Finence & $\begin{array}{l}\text { - Fineramgmt } \\
\text { - Ausbandsgoschant } \\
\text { - Corporile Finance }\end{array}$ & - Corporade Finance \\
\hline Typ & $\begin{array}{l}\text { Junges Unter- } \\
\text { nehmen stark } \\
\text { Wachsend }\end{array}$ & $\begin{array}{l}\text { - Etabliertes } \\
\text { auslandsintensives } \\
\text { Start-up" }\end{array}$ & - Globeler Spezintist & $\begin{array}{l}\text { Traditionsunter- } \\
\text { nehmen, grob mit } \\
\text { Wechatumsproble- } \\
\text { men }\end{array}$ & $\begin{array}{l}\text { Reifes, netional } \\
\text { operierendes } \\
\text { Untemehmen }\end{array}$ \\
\hline
\end{tabular}

Abbildung 38: Bedarfsprofile je Kundensegment

Quelle: Bufka, Jürgen / Eichelmann, Thomas (2002), S. 133 


\section{Anhang 7: Kundensegmentierung - Kriterientypologisierung}

\begin{tabular}{|c|c|c|c|c|c|}
\hline \multicolumn{2}{|c|}{$\begin{array}{c}\text { sozk-Jionomische } \\
\text { Kitcien }\end{array}$} & \multicolumn{2}{|c|}{$\begin{array}{l}\text { peychographische } \\
\text { Kriterien }\end{array}$} & \multicolumn{2}{|c|}{$\begin{array}{c}\text { Verhalitensorientierte } \\
\text { Kuteris }\end{array}$} \\
\hline $\begin{array}{l}\text { Privat- } \\
\text { kunden }\end{array}$ & $\begin{array}{l}\text { Fimen- } \\
\text { kunden }\end{array}$ & $\begin{array}{l}\text { Privat- } \\
\text { kunden }\end{array}$ & $\begin{array}{l}\text { Firmen- } \\
\text { kunden }\end{array}$ & $\begin{array}{l}\text { Privat- } \\
\text { kunden }\end{array}$ & $\begin{array}{l}\text { Firmen- } \\
\text { kunden }\end{array}$ \\
\hline \multirow{2}{*}{$\begin{array}{l}\text { Fami-ien- } \\
\text { Isbenanyldo } \\
\text { - Ater } \\
\text { - Geschlecht } \\
\text { - Familien- } \\
\text { stand } \\
\text { Anzahd der } \\
\text { Kinder }\end{array}$} & \multirow{2}{*}{$\begin{array}{l}\text { Unitorneh- } \\
\text { menopheae } \\
\text { - Grondung } \\
\text { - Wechstums- } \\
\text { phase } \\
\text { - Konsol- } \\
\text { dierung } \\
\text { - Lquidation }\end{array}$} & \multirow{4}{*}{$\begin{array}{l}\text { Algemeine } \\
\text { Pervōn- } \\
\text { lichtreits- } \\
\text { mertunale } \\
\text { - Activitalen } \\
\text { - Interessen } \\
\text { - Meinungen } \\
\text { Lebensstil } \\
\text { - Soziale } \\
\text { Orientierung } \\
\text { - Wegnis- } \\
\text { freudigkeit } \\
\text { Person- } \\
\text { lichkeits- } \\
\text { imventere }\end{array}$} & \multirow{2}{*}{$\begin{array}{l}\text { - Produzieren- } \\
\text { des Gewerbe } \\
\text { - Hendel } \\
\text { - Dienstlel- }\end{array}$} & \multicolumn{2}{|c|}{ 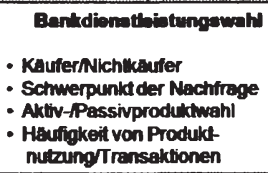 } \\
\hline & & & & \multirow{2}{*}{\multicolumn{2}{|c|}{ 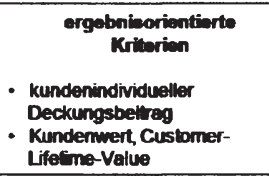 }} \\
\hline \multirow{3}{*}{$\begin{array}{l}\text { sorinh } \\
\text { Schich } \\
\text { - Enkommen } \\
\text { - Vermogen } \\
\text { - Schubidung } \\
\text { - Beruf }\end{array}$} & \multirow{3}{*}{ 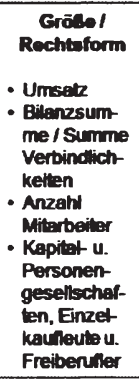 } & & $\begin{array}{l}\text { shungsunter- } \\
\text { nohmen }\end{array}$ & & \\
\hline & & & & \multirow{3}{*}{\multicolumn{2}{|c|}{ 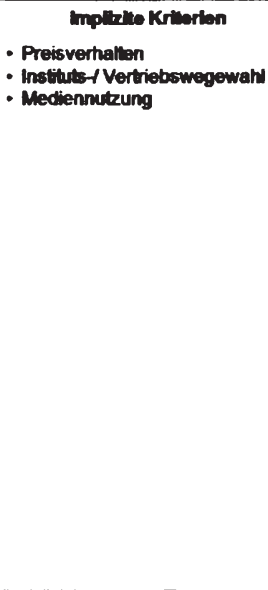 }} \\
\hline & & \multirow{2}{*}{\multicolumn{2}{|c|}{ 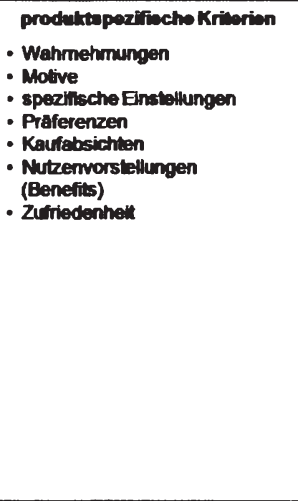 }} & & \\
\hline \multicolumn{2}{|c|}{ 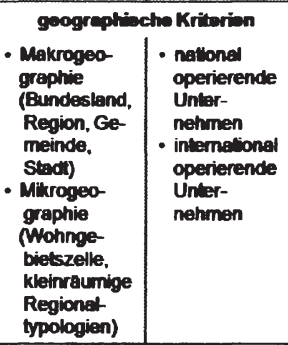 } & & & & \\
\hline & & $\begin{array}{l}\text { malidine } \\
\text { Krite }\end{array}$ & 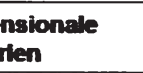 & & \\
\hline & & $\begin{array}{l}\text { Privat- } \\
\text { kunden }\end{array}$ & $\begin{array}{l}\text { Firmen- } \\
\text { kunden }\end{array}$ & & \\
\hline & & $\begin{array}{l}\text { mohrste } \\
\text { mohrdime } \\
\text { segmen }\end{array}$ & $\begin{array}{l}\text { for und } \\
\text { noional } \\
\text { torung }\end{array}$ & & \\
\hline
\end{tabular}

Abbildung 39: Übersicht - Typologisierung der Segmentierungskriterien

Quelle: Keller, Jens (2006), S. 9 


\section{Anhang 8: Portfolio-Methode der Boston Consulting Group}

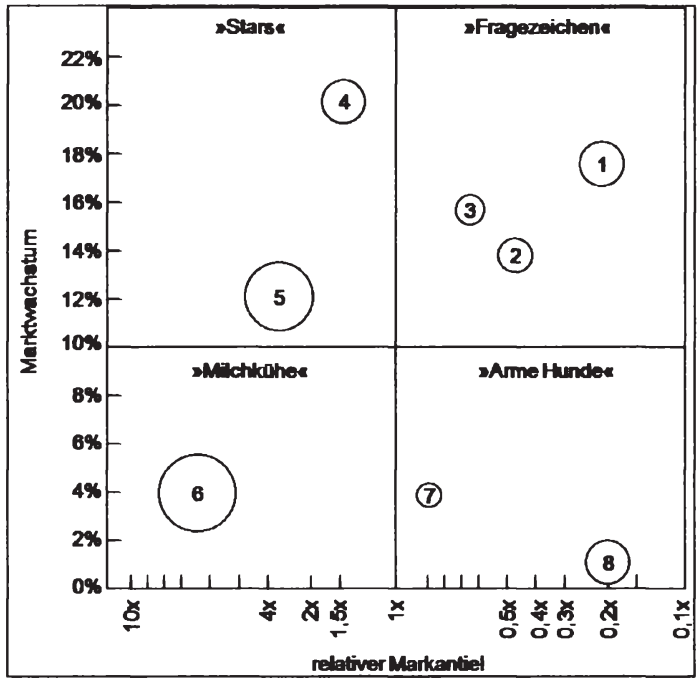

Abbildung 40: BCG-Matrix

Quelle: Bliemel, Friedhelm / Kotler, Philip (2005), S. 118

\begin{tabular}{|c|c|c|}
\hline $\begin{array}{l}\text { Feldbezeichnung / } \\
\text { SGE-Typ }\end{array}$ & Feldcharakteristik & Mögliche Normstrategie \\
\hline Question Marks & $\begin{array}{l}\text { - operiert auf Wachstumsmarkt } \\
\text { - geringer relativer Marktanteil } \\
\text { - hierunter fallen SGEs in der Gründungs- } \\
\text { phase } \\
\text { - hoher kontinuierlicher Aufwand erforder- } \\
\text { lich, um Marktwachstum zu übertreffen } \\
\text { - kein positiver Cash flow }\end{array}$ & $\begin{array}{l}\text { Ausbauen } \\
\rightarrow \text { Marktanteil der Geschäftseinheit soll } \\
\text { vergrößert werden }\end{array}$ \\
\hline Stars & $\begin{array}{l}\text { - starkes Marktwachstum } \\
\text { - hoher relativer Marktanteil } \\
\text { - entwickeln sich aus erfolgreichen } \\
\text { Question Marks } \\
\text { - kontinuierlicher Aufwand, um mit } \\
\text { Marktwachstum Schritt zu halten } \\
\text { - erste Gewinne } \\
\end{array}$ & $\begin{array}{l}\text { Erhalten } \\
\rightarrow \text { Marktanteil soll auf gegenwärtigem } \\
\text { Niveau gehalten werden }\end{array}$ \\
\hline Cash Cows & $\begin{array}{l}\text { - } \text { moderates Marktwachstum } \\
\text { - hoher relativer Marktanteil } \\
\text { - geringer Investitionsaufwand } \\
\text { - Vorteile durch Fixkostendegression } \\
\text { - hohe Gewinne }\end{array}$ & $\begin{array}{l}\text { Emten } \\
\rightarrow \text { Gewinnabschöpfung, Investitionen } \\
\text { verringern }\end{array}$ \\
\hline Poor Dogs & $\begin{array}{l}\text { - stagnierendes Marktwachstum } \\
\text { - geringer relativer Marktanteil } \\
\text { - erwirtschaften nur noch geringe Gewin- } \\
\text { ne, bzw. sind bereits defizitär }\end{array}$ & $\begin{array}{l}\text { - Abstoßen } \\
\rightarrow \text { Veräußerung oder Aufgabe der } \\
\text { Geschäftseinheit }\end{array}$ \\
\hline
\end{tabular}

\section{Tabelle 11: Die Positionierungsfelder der BCG-Matrix}

Quelle: Bliemel, Friedhelm / Kotler, Philip (2005), S. 118 f. 


\section{Anhang 9: Portfolio-Methode von McKinsey}

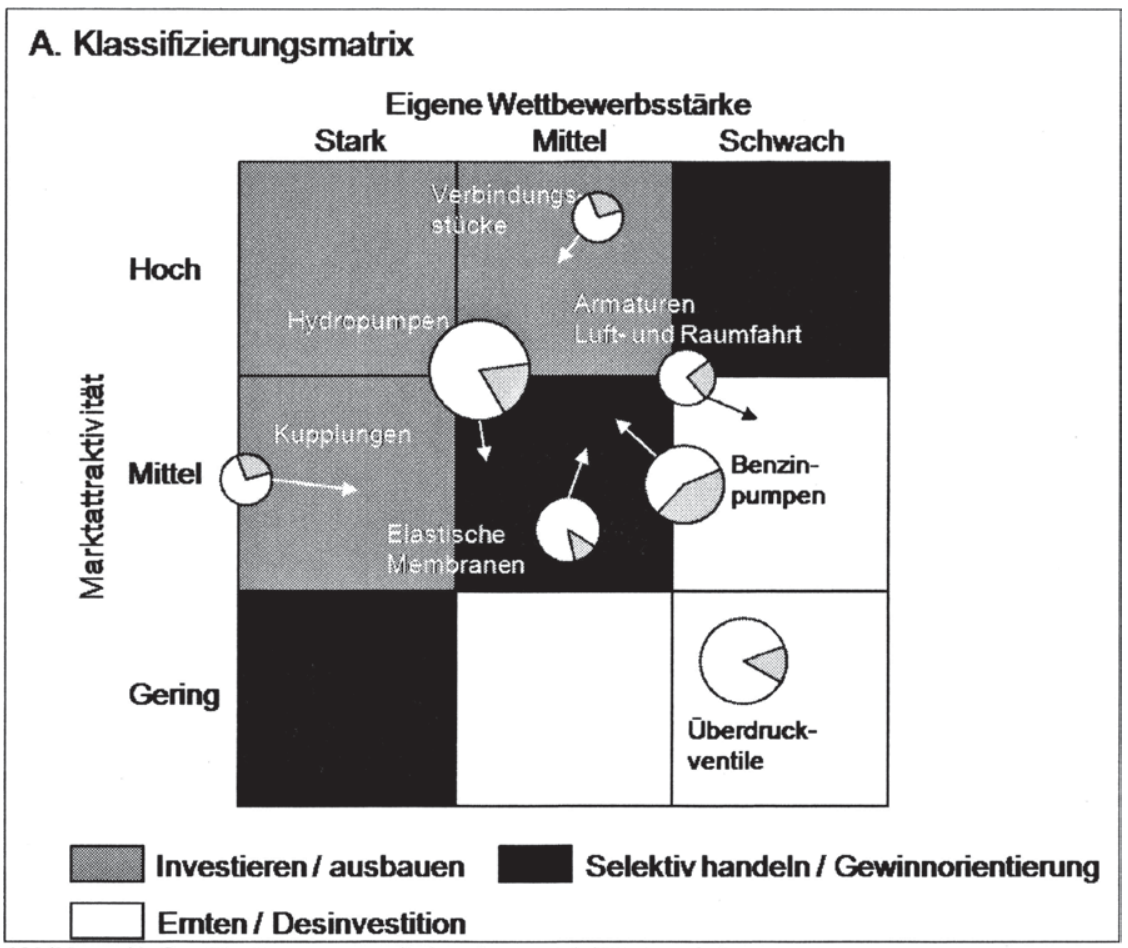

Abbildung 41: Das Wettbewerbsvorteils-Marktattraktivitäts-Portfolio

Quelle: Bliemel, Friedhelm / Kotler, Philip (2005), S. 122 


\section{B. Strategiezuordnung}

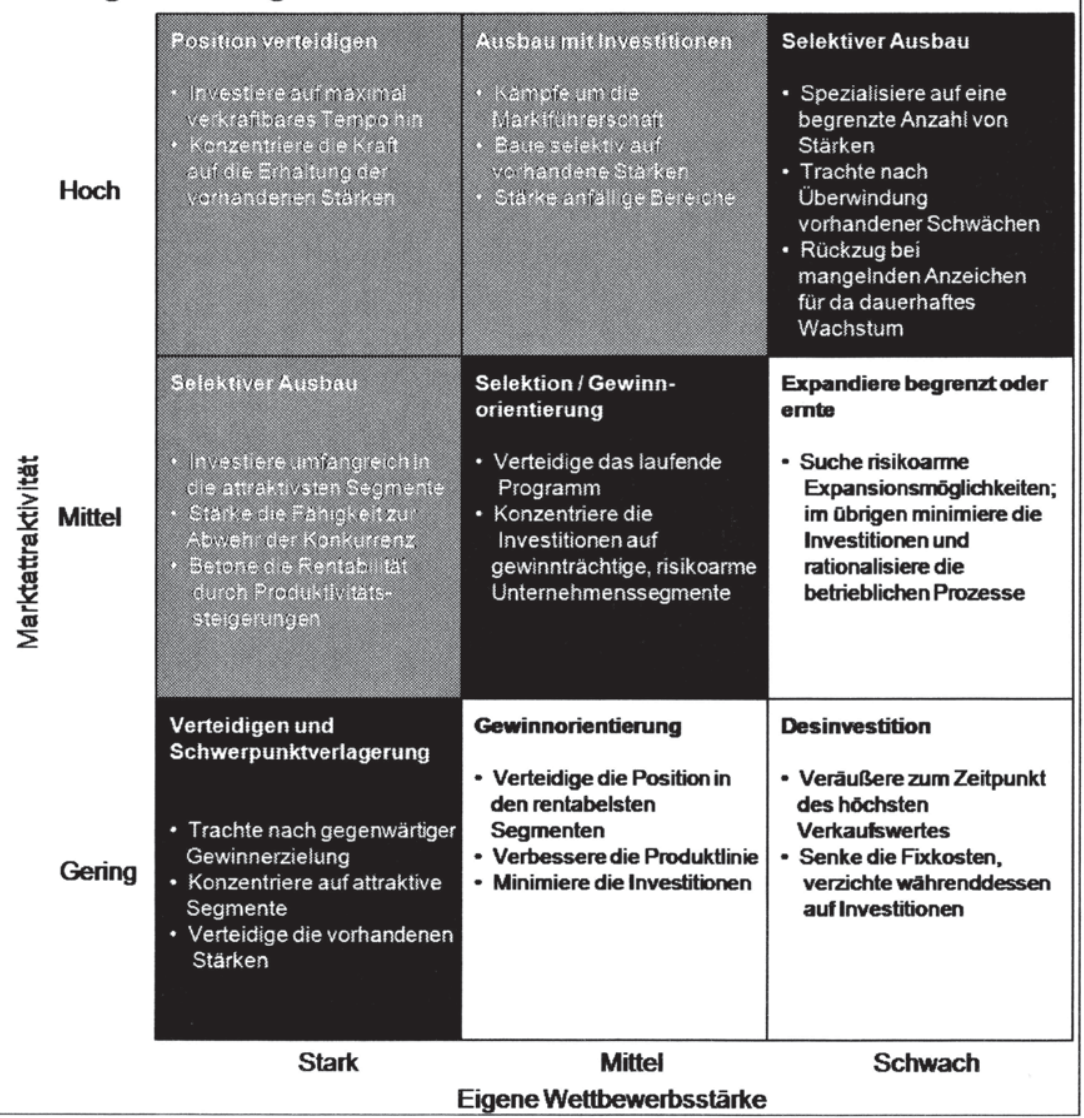

Abbildung 42: Normstrategien des McKinsey-Portfolios

Quelle: Bliemel, Friedhelm / Kotler, Philip (2005), S. 122 


\begin{tabular}{|c|c|c|c|c|}
\hline & & $\begin{array}{l}\text { Gewich- } \\
\text { tung }\end{array}$ & $\begin{array}{l}\text { Punktwert } \\
(1-5)\end{array}$ & $\begin{array}{l}\text { gewichte- } \\
\text { ter Wert }\end{array}$ \\
\hline \multirow{11}{*}{$\begin{array}{l}\text { Marktattrak- } \\
\text { tivität }\end{array}$} & \multirow{10}{*}{$\begin{array}{l}\text { Marktgröße } \\
\text { Jährliche Wachstumsrate } \\
\text { Gewinnspannen in der Branche } \\
\text { Wettbewerbsintensität } \\
\text { technologische Erfordernisse } \\
\text { Inflationsanfälligkeit } \\
\text { Energiebedarf } \\
\text { Umwelteinwirkungen } \\
\text { gesellschaftliches/politisch-rechtliches Umfeld }\end{array}$} & 0,20 & \multirow{10}{*}{$\begin{array}{c}4,00 \\
5,00 \\
4,00 \\
2,00 \\
4,00 \\
3,00 \\
2,00 \\
3,00 \\
\text { muss } \\
\text { akzeptabel } \\
\text { sein }\end{array}$} & 0,80 \\
\hline & & 0,20 & & 1,00 \\
\hline & & 0,15 & & 0,60 \\
\hline & & 0,15 & & 0,30 \\
\hline & & 0,15 & & 0,60 \\
\hline & & 0,05 & & 0,15 \\
\hline & & 0,05 & & 0,10 \\
\hline & & 0,05 & & 0,15 \\
\hline & & & & \\
\hline & & 1,00 & & 3,70 \\
\hline & & $\begin{array}{l}\text { Gewich- } \\
\text { tung }\end{array}$ & $\begin{array}{l}\text { Punktwert } \\
(1-5)\end{array}$ & $\begin{array}{l}\text { gewichte- } \\
\text { ter Wert }\end{array}$ \\
\hline \multirow{13}{*}{$\begin{array}{l}\text { Eigene Wett- } \\
\text { bewerbsstärke }\end{array}$} & Marktanteil & 0,10 & 4,00 & 0,40 \\
\hline & Wachstum des Marktanteils & 0,15 & 2,00 & 0,30 \\
\hline & Produktqualität & 0,10 & 4,00 & 0,40 \\
\hline & Markenimage & 0,10 & 5,00 & 0,50 \\
\hline & Distributionsnetz & 0,05 & 4,00 & 0,20 \\
\hline & Effektivität der Absatzförderung & 0,05 & 3,00 & 0,15 \\
\hline & Produktionskapazität & 0,05 & 3,00 & 0,15 \\
\hline & Produktionseffizienz & 0,05 & 2,00 & 0,10 \\
\hline & Stückkosten & 0,15 & 3,00 & 0,45 \\
\hline & Materialversorgung & 0,05 & 5,00 & 0,25 \\
\hline & Leistungsfähigkeit in F \& E & 0,10 & 3,00 & 0,30 \\
\hline & \multirow[t]{2}{*}{ Qualifikation der Führungskräfte } & 0,05 & \multirow[t]{2}{*}{4,00} & 0,20 \\
\hline & & 1,00 & & 3,40 \\
\hline
\end{tabular}

Tabelle 12: Multifaktoren-Scoring nach McKinsey

Quelle: Bliemel, Friedhelm / Kotler, Philip (2005), S. 123 
Anhang 10: Portfolio-Methode nach SCHRÖDER

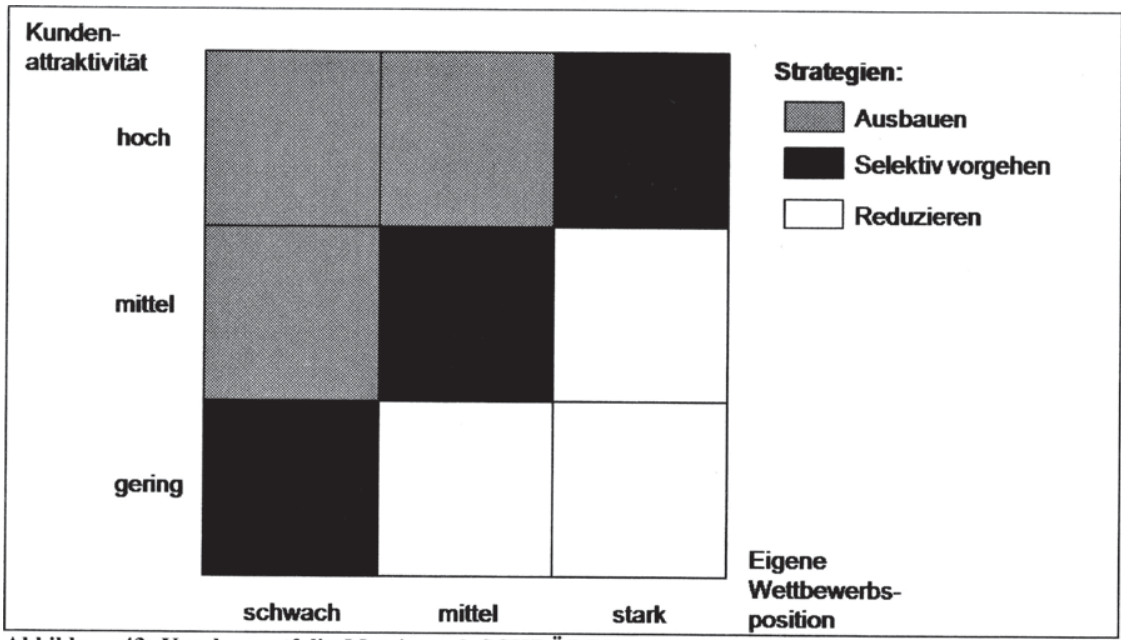

Abbildung 43: Kundenportfolio-Matrix nach SCHRÖDER

Quelle: Schröder, Gustav Adolf (2001) 


\section{Anhang 11: Segmentierungskriterien zur Potenzialerhebung}

\begin{tabular}{|c|c|}
\hline \multicolumn{2}{|l|}{ Allgemeine Kriterien } \\
\hline \multicolumn{2}{|l|}{ - Bankverbindung - Entwicklungspotenzial } \\
\hline \multicolumn{2}{|l|}{ - Einschärzung Entwicklung Deckungsbeitrag } \\
\hline Kriterien - Bexiehung zum Unternehmen & Kriterien - Beziehung zum Unternehmer \\
\hline Potenzialanalyse - Betriebliche Bedarfsfelder & Potenzialanalyse - Private Bedarfsfelder \\
\hline \multirow{3}{*}{$\begin{array}{l}\text { - Bedarfsfeld Liquidität und Zahlungsverkehr } \\
\text { Zahlungsverkehr: } \\
\text { - Kontokorrent } \\
\text { - Auslandszahlungsverkehr } \\
\text { - eBanking } \\
\text { - Kreditkartengeschäft } \\
\text { Kurzfristfinanzierung: } \\
\text { - Kontokorrent- / Termingeldkredit } \\
\text { - Factoring } \\
\text { - Avalkredit }\end{array}$} & $\begin{array}{l}\text { Bedarfsfeld Zahlungsverkehr } \\
\text { - Kontokorrent } \\
\text { - eBanking } \\
\text { - Kreditkartengeschäft }\end{array}$ \\
\hline & $\begin{array}{l}\text { Bedarfsfeld Immobilien und Finanzierung } \\
\text { - Dispositionskredit } \\
\text { - Private Anschaffungsdarlehen } \\
\text { - Wohnbaufinanzierungen }\end{array}$ \\
\hline & $\begin{array}{l}\text { Bedarfsfeld private Vermögensanlage } \\
\text { - Tages- und Festgeld }\end{array}$ \\
\hline \multirow{2}{*}{$\begin{array}{l}\text { - Bedarfsfeld Investition und Finanzierung } \\
\text { Mittel- / Langfristfinanzierung: } \\
\text { - Bankdarlehen } \\
\text { - offentliche Förderprogramme } \\
\text { - Leasing } \\
\text { - Vermittlung von Darlehen } \\
\text { - Wahrungskredite }\end{array}$} & $\begin{array}{l}\text { - Wertpapieranlage Vermögensverwalıung } \\
\text { - Bausparen } \\
\text { - Immobilien }\end{array}$ \\
\hline & \multirow[t]{3}{*}{$\begin{array}{l}\text { Bedarfsfeld Absicherung und Vorsorge } \\
\text { - Sachversicherungen } \\
\text { - Lebensversicherungen } \\
\text { - Krankenversicherungen }\end{array}$} \\
\hline $\begin{array}{l}\text { - Bedarfsfeld Vermögensanlage } \\
\text { Kurzfristanlage: } \\
\text { - Tages- und Festgeld } \\
\text { Langfristanlage: } \\
\text { - Wertpapieranlage /Vermögensvenwaltung }\end{array}$ & \\
\hline $\begin{array}{l}\text { - Bedarfsfeld Risiko und Absicherung } \\
\text { Absicherung: } \\
\text { - Sachversicherungen } \\
\text { Vorsorge: } \\
\text { - Betriebliche Altersvorsorge }\end{array}$ & \\
\hline \multirow{3}{*}{\multicolumn{2}{|c|}{$\begin{array}{l}\text { Branchensituation } \\
\text { - Aktuelle Umsatzprognose } \\
\text { - Kundenprognose im Vergleich zur Branche }\end{array}$}} \\
\hline & \\
\hline & \\
\hline $\begin{array}{l}\text { Volkswirtschaftliche Rahmenparameter - } \\
\text { Unternehmen }\end{array}$ & $\begin{array}{l}\text { Volkswirtschaftliche Rahmenparameter - } \\
\text { Unternehmer }\end{array}$ \\
\hline \multirow[t]{2}{*}{ KfW-ifo-Mittelstandsbarometer Erwartungen } & - Prognose - Kaufkraftkennziffer (regional) \\
\hline & - Prognose - Entwicklung der Sparquote \\
\hline
\end{tabular}

Tabelle 13: Segmentierungskriterien zur Potenzialerhebung 


\section{Anhang 12: Eigenentwickeltes Segmentierungstool}

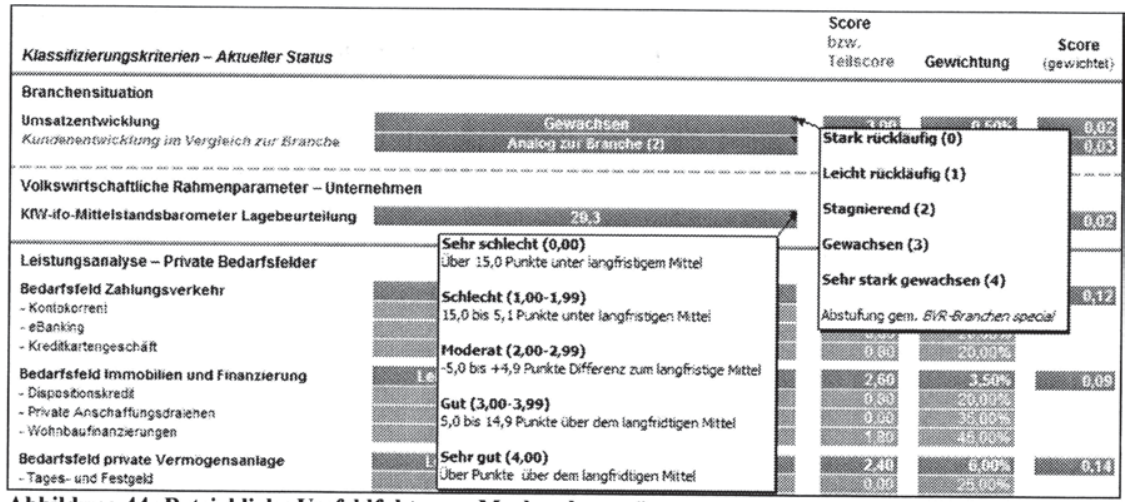

Abbildung 44: Betriebliche Umfeldfaktoren: Merkmalausprägungen

Quelle: Eigenentwickeltes Segmentierungstool

\begin{tabular}{|c|c|c|c|c|}
\hline \multicolumn{2}{|l|}{ Klassifizierungskriterien - Aktueller Status } & \multicolumn{2}{|c|}{$\begin{array}{l}\text { Score } \\
\text { brw. } \\
\text { Teilscore Gewichtung }\end{array}$} & $\begin{array}{c}\text { Score } \\
\text { (gewichtet) }\end{array}$ \\
\hline \multicolumn{2}{|c|}{ Volkswirtschaftliche Rahmenparameter - Unternehmer } & \multirow{4}{*}{\multicolumn{2}{|c|}{$\begin{array}{l}\text { Sehr schilecht }(0,00) \\
\text { inter } 92,5 \text { Indexpurkter } \\
\text { Schlecht }(1,00-1,99) \\
92,5 \text { bis } 97,4 \text { thdexpunkte } \\
\text { Durchschnittlich }(2,00-2,99) \\
97,5 \text { bis } 102,4 \text { Indexpurkte } \\
\text { Gut }(3,00-3,99) \\
102,5 \text { bis } 107,4 \text { Indexpunikte } \\
\text { Sehr gut }(4,00) \\
\text { Ab } 107,5 \text { Indexpurkte }\end{array}$}} & \\
\hline \multirow{3}{*}{$\begin{array}{l}\text { Kaufkraftkennziffer (regional) } \\
\text { Sparquote private Haushalte }\end{array}$} & 110.9 & & & 302 \\
\hline & 10085 & & & 0.04 \\
\hline & $\begin{array}{l}\text { Sehr niedrig }(0,00) \\
<9,50 \% \\
\text { Miedrig }(1,00-1,99) \\
9,50 \% \text { bis } 10,49 \% \\
\text { Stagnierend }(2,00 \cdot 2,99) \\
10,50 \% \text { bis } 11,49 \% \\
\text { Hoch }(3,00-3,99) \\
12,50 \% \text { bis } 12,49 \% \\
\text { Sehr hoch }(4,00) \\
>12,50 \% \\
\end{array}$ & & & \\
\hline
\end{tabular}

Abbildung 45: Private Umfeldfaktoren: Merkmalsausprägungen

Quelle: Eigenentwickeltes Segmentierungstool 


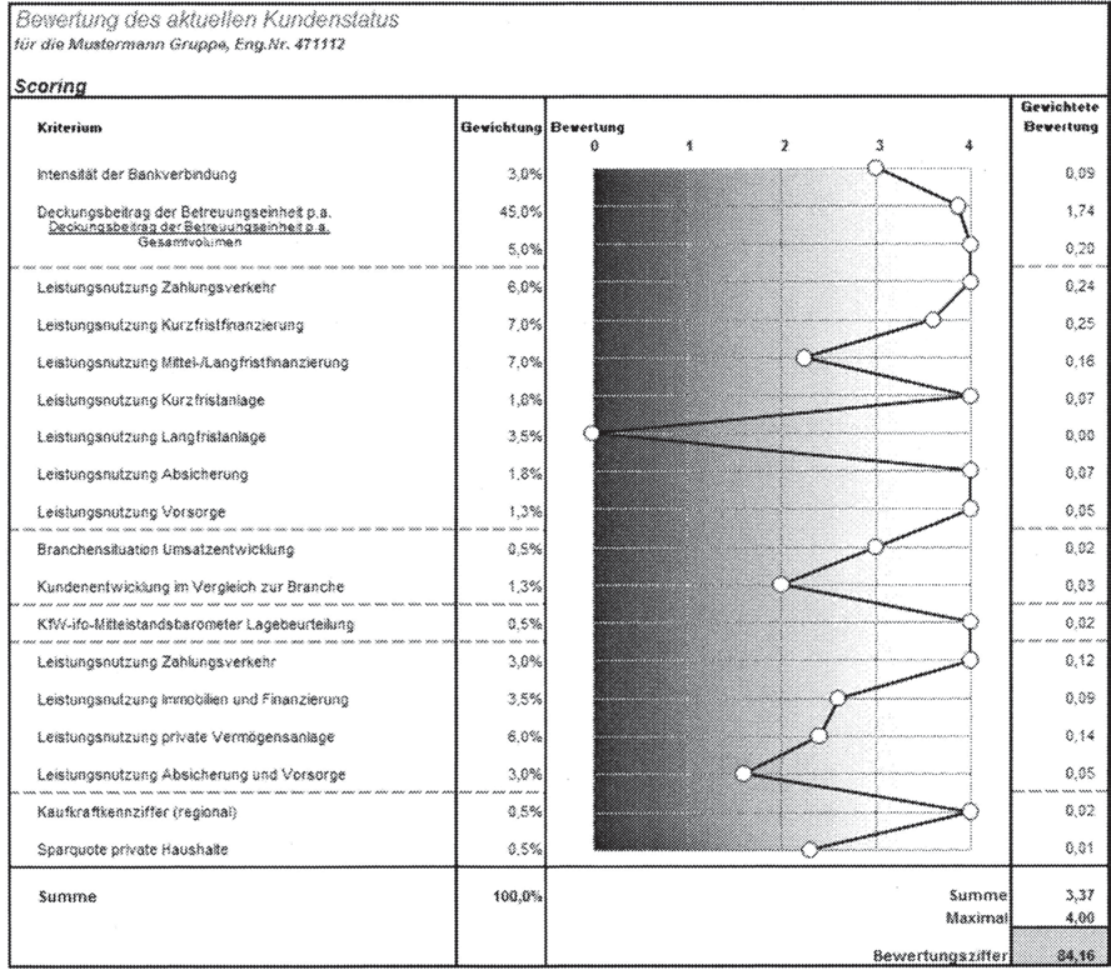

Abbildung 46: Bewertungsprofil Statusscoring 


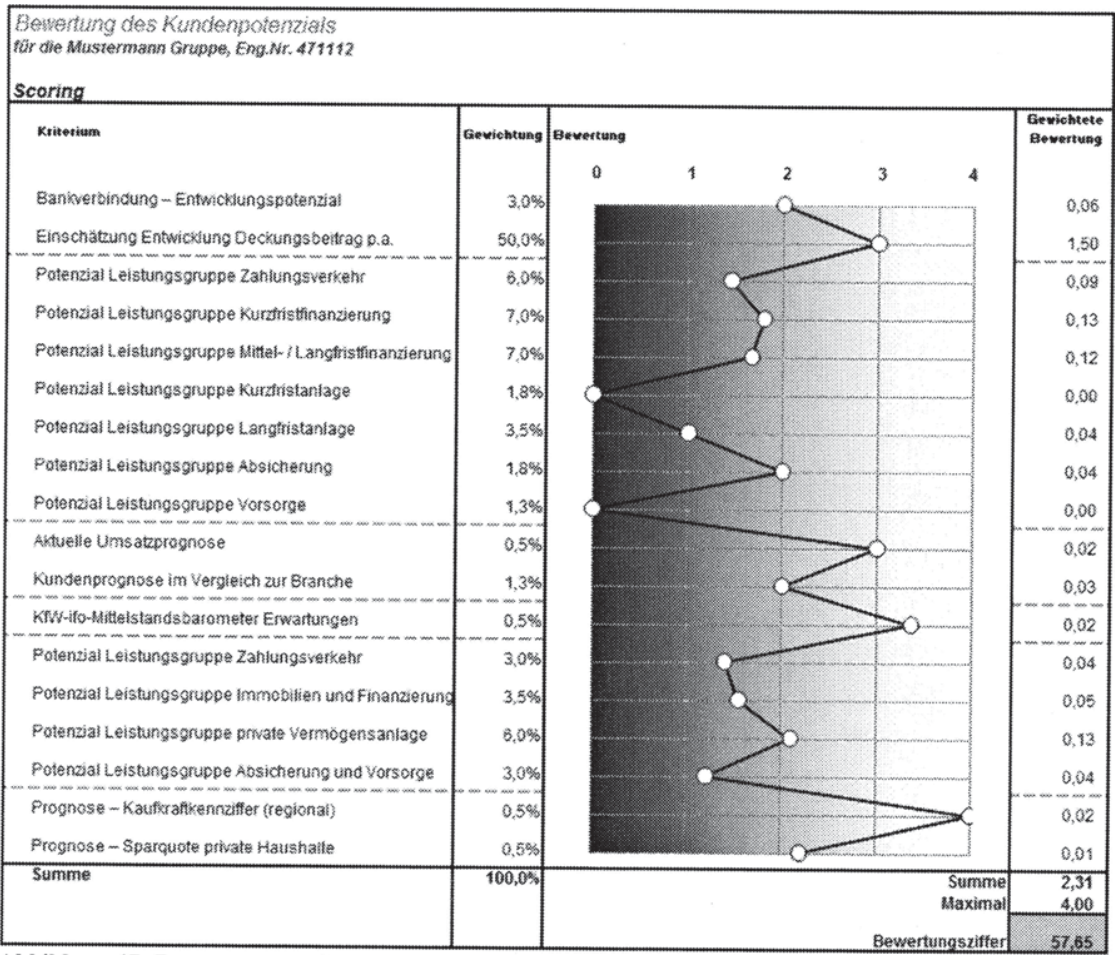

Abbildung 47: Bewertungsprofil Potenzialscoring 


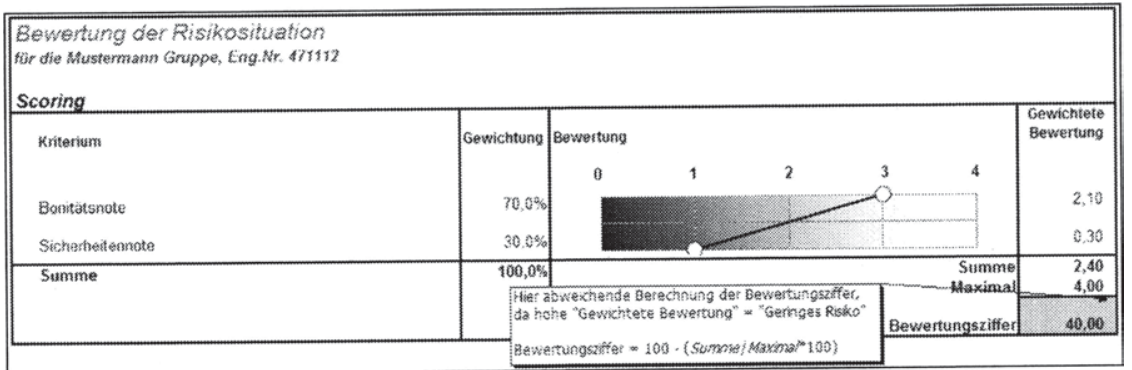

\section{Abbildung 48: Bewertungsprofil Risikoscoring}

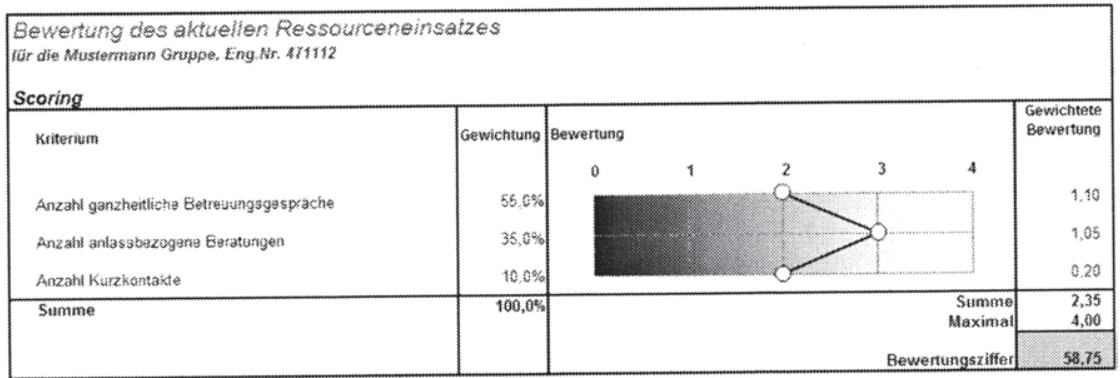

Abbildung 49: Bewertungsprofil Ressourcenscoring

Quelle: Eigenentwickeltes Segmentierungstool 


\section{Anhang 13: Fragebogen und Anschreiben}

\section{Unternehmensbefragung zum Thema: „Optimierung des Leistungsangebotes in der Firmenkundenbetreuung}

\section{Allgemeines:}

1.1 In welchem Sektor ist Ihr Unternehmen tătig?

$\square$ Produktion

$\square \quad$ Baugewerbe

$\square$ Handel

$\square \quad$ Freie Berufe

$\square$ Dienstleistungen

1.2 In welcher Umsatzgrößenklasse befand sich Ihr Unternehmen im letzten Geschäftsjahr?

$\square \quad$ unter $250.000 €$

$\square \quad 250.000 €$ bis unter $500.000 €$

$\square \quad 2.500 .000 €$ bis unter $5.000 .000 €$

$\square 00.000 €$ bis unter $1.000 .000 €$

$\square \quad 5.000 .000 €$ bis unter $10.000 .000 €$

$\square \quad 1.000 .000 €$ bis unter $2.500 .000 €$

10.000.000€ bis unter $50.000 .000 €$

1.3 Die Eigenkapitalquote (Eigenkapital im Verhältnis zur Bilanzsumme) Ihres Unternehmens betrăgt?

$\square \quad$ unter $10 \%$

ㅁ $10 \%$ bis unter $20 \%$

$20 \%$ bis unter $30 \%$

$\square$ über $30 \%$

1.4 $\mathrm{Zu}$ wie vielen Banken unterhalten Sie aktuell eine aktive Kontoverbindung?

$\mathrm{Zu}$ Bank(en).

1.5 $\mathrm{Zu}$ welcher Institutsgruppe gehört Ihre Hausbank?

$\square$ Volks- und Raiffeisenbanken

$\square$ Großbanken

$\square$ Sonstige

$\square$ Sparkassen

$\square$ Privatbanken

1.6 Wie zufrieden sind Sie insgesamt mit Ihrer Hausbank? Wenn Sie außerdem noch auf andere Dienstleister zurückgreifen, geben Sie bitte auch hier Ihre Gesamtzufriedenheit an.

Für den Fall, dass Sie in einer der genannten Kategorien mit mehr als einem Anbieter zusammenarbeiten (z.B. zwei Unternehmensberater), votieren Sie bitte für denjenigen mit der intensiveren Geschăftsbeziehung zu Ihrem Unternehmen.

\begin{tabular}{|c|c|c|c|c|c|c|c|}
\hline \multirow[b]{3}{*}{ Dienstleister } & \multicolumn{7}{|c|}{ Zufriedenheit } \\
\hline & \multicolumn{7}{|c|}{$\begin{array}{c}1=\text { sehr unzufrieden } \\
7=\text { sehr zufrieden }\end{array}$} \\
\hline & 1 & 2 & 3 & 4 & 5 & 6 & 7 \\
\hline Hausbank & $\square$ & $\square$ & $\square$ & $\square$ & $\square$ & $\square$ & $\square$ \\
\hline Zweitkreditinstitut & $\square$ & $\bar{\square}$ & $\square$ & $\bar{\square}$ & $\bar{\square}$ & $\bar{\square}$ & $\bar{\square}$ \\
\hline Finanzdienstleister & $\square$ & $\square$ & $\square$ & $\square$ & $\square$ & $\square$ & $\square$ \\
\hline Steuerberater & $\square$ & $\square$ & $\square$ & $\square$ & $\square$ & $\square$ & $\square$ \\
\hline Wirtschaftsprüfer & $\square$ & $\square$ & $\square$ & $\square$ & $\square$ & $\square$ & $\square$ \\
\hline Unternehmensberater & $\square$ & $\square$ & $\square$ & $\square$ & $\square$ & $\square$ & $\square$ \\
\hline
\end{tabular}




\section{Leistungsangebot im Firmenkundengeschäft}

2.1 Bitte geben Sie für die in Frage 1.6 bewerteten Unternehmen folgendes an:

- Welche der nachstehenden Leistungsangebote sind Ihnen schon einmal aktiv angeboten worden? Kennzeichnen Sie bitte den/die entsprechenden Dienstleister (Mehrfachnennungen erlaubt).

- Bitte markieren Sie in der Spalte daneben, die Dienstleistungen die Sie schon einmal in Anspruch genommen haben und kennzeichnen Sie den ausgewählten Anbieter (Einfachnennung).

Für den Fall, dass Sie einige Dienstleistungen bereits bei mehreren Anbietern genutzt haben, wählen Sie bitte denjenigen aus, mit dem Sie vorrangig zusammengearbeitet haben.

\begin{tabular}{|c|c|c|c|c|c|c|c|c|c|c|c|c|}
\hline \multirow[b]{2}{*}{ Dienstleistung } & \multicolumn{6}{|c|}{ Leistungsangebot } & \multicolumn{6}{|c|}{$\begin{array}{c}\text { Leistungs- } \\
\text { inanspruchnahme }\end{array}$} \\
\hline & 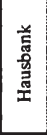 & 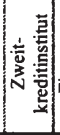 & 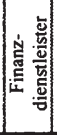 & 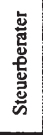 & 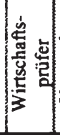 & 至 总 & $\begin{array}{l}\text { है } \\
\text { औूँ } \\
\text { ज्ञ }\end{array}$ & 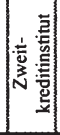 & 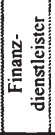 & 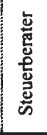 & 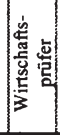 & 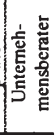 \\
\hline $\begin{array}{l}\text { Bedarfsfeld: Liquiditat und Zahlungsverkehr } \\
\text { Betriebsmittelkredite } \\
\text { Elektronischer Zahlungsverkehr } \\
\text { Cash Management } \\
\text { Auslandszahlungsverkehr }\end{array}$ & $\begin{array}{l}\square \\
\square \\
\square \\
\square\end{array}$ & $\begin{array}{l}\square \\
\square \\
\square \\
\square\end{array}$ & $\begin{array}{l}\square \\
\square \\
\square \\
\square\end{array}$ & $\begin{array}{l}\square \\
\square \\
\square \\
\square\end{array}$ & $\begin{array}{l}\square \\
\square \\
\square \\
\square\end{array}$ & $\begin{array}{l}\square \\
\square \\
\square \\
\square\end{array}$ & $\begin{array}{l}\square \\
\square \\
\square \\
\square\end{array}$ & $\begin{array}{l}\square \\
\square \\
\square \\
\square\end{array}$ & $\begin{array}{l}\square \\
\square \\
\square \\
\square\end{array}$ & $\begin{array}{l}\square \\
\square \\
\square \\
\square\end{array}$ & $\begin{array}{l}\square \\
\square \\
\square \\
\square\end{array}$ & $\begin{array}{l}\square \\
\square \\
\square \\
\square\end{array}$ \\
\hline $\begin{array}{l}\text { Bedarfsfeld: Investition und Finanzierung } \\
\text { Investitionskredite } \\
\text { Vermittlung öffentlicher Fördermittel } \\
\text { Hypothekenvermittlung } \\
\text { Immobilienvermittlung } \\
\text { Leasing } \\
\text { Im- und Exportfinanzierung } \\
\text { Bürgschafts- und Garantiegeschäft } \\
\text { Mezzanine Finanzierung }\end{array}$ & $\begin{array}{l}\square \\
\square \\
\square \\
\square \\
\square \\
\square \\
\square \\
\square\end{array}$ & $\begin{array}{l}\square \\
\square \\
\square \\
\square \\
\square \\
\square \\
\square \\
\square\end{array}$ & $\begin{array}{l}\square \\
\square \\
\square \\
\square \\
\square \\
\square \\
\square \\
\square\end{array}$ & $\begin{array}{l}\square \\
\square \\
\square \\
\square \\
\square \\
\square \\
\square \\
\square\end{array}$ & $\begin{array}{l}\square \\
\square \\
\square \\
\square \\
\square \\
\square \\
\square \\
\square\end{array}$ & $\begin{array}{l}\square \\
\square \\
\square \\
\square \\
\square \\
\square \\
\square \\
\square\end{array}$ & $\begin{array}{l}\square \\
\square \\
\square \\
\square \\
\square \\
\square \\
\square \\
\square\end{array}$ & $\begin{array}{l}\square \\
\square \\
\square \\
\square \\
\square \\
\square \\
\square \\
\square\end{array}$ & $\begin{array}{l}\square \\
\square \\
\square \\
\square \\
\square \\
\square \\
\square \\
\square\end{array}$ & $\begin{array}{l}\square \\
\square \\
\square \\
\square \\
\square \\
\square \\
\square \\
\square\end{array}$ & $\begin{array}{l}\square \\
\square \\
\square \\
\square \\
\square \\
\square \\
\square \\
\square\end{array}$ & $\begin{array}{l}\square \\
\square \\
\square \\
\square \\
\square \\
\square \\
\square \\
\square\end{array}$ \\
\hline $\begin{array}{l}\text { Bedarfsfeld: Risiko und Absicherung } \\
\text { Forderungsmanagement } \\
\text { Zins- und Wăhrungsmanagement } \\
\text { Sachversicherungen } \\
\text { Lebensversicherungen } \\
\text { Betriebliche Altersvorsorge }\end{array}$ & $\begin{array}{l}\square \\
\square \\
\square \\
\square \\
\square\end{array}$ & $\begin{array}{l}\square \\
\square \\
\square \\
\square \\
\square\end{array}$ & $\begin{array}{l}\square \\
\square \\
\square \\
\square \\
\square\end{array}$ & $\begin{array}{l}\square \\
\square \\
\square \\
\square \\
\square\end{array}$ & $\begin{array}{l}\square \\
\square \\
\square \\
\square \\
\square\end{array}$ & $\begin{array}{l}\square \\
\square \\
\square \\
\square \\
\square\end{array}$ & $\begin{array}{l}\square \\
\square \\
\square \\
\square \\
\square\end{array}$ & $\begin{array}{l}\square \\
\square \\
\square \\
\square \\
\square\end{array}$ & $\begin{array}{l}\square \\
\square \\
\square \\
\square \\
\square\end{array}$ & $\begin{array}{l}\square \\
\square \\
\square \\
\square \\
\square\end{array}$ & $\begin{array}{l}\square \\
\square \\
\square \\
\square \\
\square\end{array}$ & $\begin{array}{l}\square \\
\square \\
\square \\
\square \\
\square\end{array}$ \\
\hline $\begin{array}{l}\text { Bedarfsfeld: Vermögensanlage } \\
\text { Anlagemanagement } \\
\text { Vermögensberatung } \\
\text { Private Vermögensplanung für den Unternehmer }\end{array}$ & $\begin{array}{l}\square \\
\square \\
\square \\
\end{array}$ & $\begin{array}{l}\square \\
\square \\
\square\end{array}$ & $\begin{array}{l}\square \\
\square \\
\square\end{array}$ & $\begin{array}{l}\square \\
\square \\
\square\end{array}$ & $\begin{array}{l}\square \\
\square \\
\square\end{array}$ & $\begin{array}{l}\square \\
\square \\
\square\end{array}$ & $\begin{array}{l}\square \\
\square \\
\square\end{array}$ & $\begin{array}{l}\square \\
\square \\
\square\end{array}$ & $\begin{array}{l}\square \\
\square \\
\square\end{array}$ & $\begin{array}{l}\square \\
\square \\
\square\end{array}$ & $\begin{array}{l}\square \\
\square \\
\square\end{array}$ & $\begin{array}{l}\square \\
\square \\
\square\end{array}$ \\
\hline $\begin{array}{l}\text { Bedarfsfeld: Betriebswirtschaftliche Beratung } \\
\text { Regelmäßiges ganzheitliches Strategiegespräch } \\
\text { Bilanzanalysegespräch } \\
\text { Ratingberatung } \\
\text { Stärken-/ Schwächenanalyse } \\
\text { Risikoanalyse / Risikoprofil } \\
\text { Existenzgründungsberatung } \\
\text { Unternelimensnachfolgeberatung } \\
\text { Projektbeurteilung } \\
\text { Beratung von Auslandsprojekten } \\
\text { Unterstützung bei der Investitionsplanung } \\
\text { Unterstützung bei der Erstellung einer Planbilanz } \\
\text { Unterstützung bei der Liquiditätsplanung } \\
\text { Lohnnebenkostenoptimierung } \\
\text { Workshops / Erfahrungsaustausch zu } \\
\text { betriebswirtschaftlichen Themen }\end{array}$ & $\begin{array}{l}\square \\
\square \\
\square \\
\square \\
\square \\
\square \\
\square \\
\square \\
\square \\
\square \\
\square \\
\square \\
\square \\
\square\end{array}$ & $\begin{array}{l}\square \\
\square \\
\square \\
\square \\
\square \\
\square \\
\square \\
\square \\
\square \\
\square \\
\square \\
\square \\
\square \\
\square\end{array}$ & $\begin{array}{l}\square \\
\square \\
\square \\
\square \\
\square \\
\square \\
\square \\
\square \\
\square \\
\square \\
\square \\
\square \\
\square \\
\square\end{array}$ & $\begin{array}{l}\square \\
\square \\
\square \\
\square \\
\square \\
\square \\
\square \\
\square \\
\square \\
\square \\
\square \\
\square \\
\square \\
\square\end{array}$ & $\begin{array}{l}\square \\
\square \\
\square \\
\square \\
\square \\
\square \\
\square \\
\square \\
\square \\
\square \\
\square \\
\square \\
\square \\
\square\end{array}$ & $\begin{array}{l}\square \\
\square \\
\square \\
\square \\
\square \\
\square \\
\square \\
\square \\
\square \\
\square \\
\square \\
\square \\
\square \\
\square\end{array}$ & $\begin{array}{l}\square \\
\square \\
\square \\
\square \\
\square \\
\square \\
\square \\
\square \\
\square \\
\square \\
\square \\
\square \\
\square \\
\square\end{array}$ & $\begin{array}{l}\square \\
\square \\
\square \\
\square \\
\square \\
\square \\
\square \\
\square \\
\square \\
\square \\
\square \\
\square \\
\square \\
\square\end{array}$ & $\begin{array}{l}\square \\
\square \\
\square \\
\square \\
\square \\
\square \\
\square \\
\square \\
\square \\
\square \\
\square \\
\square \\
\square \\
\square\end{array}$ & $\begin{array}{l}\square \\
\square \\
\square \\
\square \\
\square \\
\square \\
\square \\
\square \\
\square \\
\square \\
\square \\
\square \\
\square \\
\square\end{array}$ & $\begin{array}{l}\square \\
\square \\
\square \\
\square \\
\square \\
\square \\
\square \\
\square \\
\square \\
\square \\
\square \\
\square \\
\square \\
\square\end{array}$ & $\begin{array}{l}\square \\
\square \\
\square \\
\square \\
\square \\
\square \\
\square \\
\square \\
\square \\
\square \\
\square \\
\square \\
\square \\
\square\end{array}$ \\
\hline
\end{tabular}


2.2 Beurteilen Sie nun die Wichtigkeit der genannten Dienstleistungen für Ihr Unternehmen. Bitte geben Sie zusätzlich Ihre Zufriedenheit mit der Inanspruchnahme an, wenn Sie die Dienstleistung schon einmal bei Ihrer Hausbank genutzt haben.

Für den Fall, dass Sie die Dienstleistung noch nie bei Ihrer Hausbank in Anspruch genommen haben, kreuzen Sie bitte das Kästchen in der Spalte „0“ an.

\begin{tabular}{|c|c|c|c|c|c|c|c|c|c|c|c|c|c|c|c|}
\hline \multirow[b]{3}{*}{ Dienstleistung } & \multicolumn{7}{|c|}{ Wichtigkeit } & \multicolumn{8}{|c|}{ Zufriedenheit } \\
\hline & \multicolumn{7}{|c|}{$\begin{array}{c}1 \text { = äuBerst unwichtig } \\
7=\text { äuBerst wichtig }\end{array}$} & \multicolumn{8}{|c|}{$\begin{array}{c}0=\text { noch nie genutzt } \\
1=\text { sehr unzufrieden } \\
7=\text { sehr zufrieden }\end{array}$} \\
\hline & 1 & 2 & 3 & 4 & 5 & 6 & 7 & 0 & 1 & 2 & 3 & 4 & 5 & 6 & 7 \\
\hline $\begin{array}{l}\text { Bedarfsfeld: Liquiditat und Zahlungsverkehr } \\
\text { Betriebsmittelkredite } \\
\text { Elektronischer Zahlungsverkehr } \\
\text { Cash Management } \\
\text { Auslandszahlungsverkehr }\end{array}$ & $\begin{array}{l}\square \\
\square \\
\square \\
\square \\
\end{array}$ & $\begin{array}{l}\square \\
\square \\
\square \\
\square \\
\end{array}$ & $\begin{array}{l}\square \\
\square \\
\square \\
\square \\
\end{array}$ & $\begin{array}{l}\square \\
\square \\
\square \\
\square \\
\end{array}$ & $\begin{array}{l}\square \\
\square \\
\square \\
\square \\
\end{array}$ & $\begin{array}{l}\square \\
\square \\
\square \\
\square \\
\end{array}$ & $\begin{array}{l}\square \\
\square \\
\square \\
\square \\
\end{array}$ & $\begin{array}{l}\square \\
\square \\
\square \\
\square \\
\end{array}$ & $\begin{array}{l}\square \\
\square \\
\square \\
\square\end{array}$ & $\begin{array}{l}\square \\
\square \\
\square \\
\square\end{array}$ & $\begin{array}{l}\square \\
\square \\
\square \\
\square \\
\end{array}$ & $\begin{array}{l}\square \\
\square \\
\square \\
\square \\
\end{array}$ & $\begin{array}{l}\square \\
\square \\
\square \\
\square\end{array}$ & $\begin{array}{l}\square \\
\square \\
\square \\
\square \\
\end{array}$ & $\begin{array}{l}\square \\
\square \\
\square \\
\square \\
\end{array}$ \\
\hline $\begin{array}{l}\text { Bedarfsfeld: Investition und Finanzierung } \\
\text { Investitionskredite } \\
\text { Vermittlung öffentlicher Fördermittel } \\
\text { Hypothekenvermittlung } \\
\text { Immobilienvermittlung } \\
\text { Leasing } \\
\text { Im- und Exportfinanzierung } \\
\text { Bürgschafts- und Garantiegeschäft } \\
\text { Mezzanine Finanzierung }\end{array}$ & $\begin{array}{l}\square \\
\square \\
\square \\
\square \\
\square \\
\square \\
\square \\
\square \\
\end{array}$ & $\begin{array}{l}\square \\
\square \\
\square \\
\square \\
\square \\
\square \\
\square \\
\square\end{array}$ & $\begin{array}{l}\square \\
\square \\
\square \\
\square \\
\square \\
\square \\
\square \\
\square\end{array}$ & $\begin{array}{l}\square \\
\square \\
\square \\
\square \\
\square \\
\square \\
\square \\
\square\end{array}$ & $\begin{array}{l}\square \\
\square \\
\square \\
\square \\
\square \\
\square \\
\square \\
\square\end{array}$ & $\begin{array}{l}\square \\
\square \\
\square \\
\square \\
\square \\
\square \\
\square \\
\square\end{array}$ & $\begin{array}{l}\square \\
\square \\
\square \\
\square \\
\square \\
\square \\
\square \\
\square\end{array}$ & \begin{tabular}{|l|}
$\square$ \\
$\square$ \\
$\square$ \\
$\square$ \\
$\square$ \\
$\square$ \\
$\square$ \\
$\square$ \\
\end{tabular} & $\begin{array}{l}\square \\
\square \\
\square \\
\square \\
\square \\
\square \\
\square \\
\square\end{array}$ & $\begin{array}{l}\square \\
\square \\
\square \\
\square \\
\square \\
\square \\
\square \\
\square\end{array}$ & $\begin{array}{l}\square \\
\square \\
\square \\
\square \\
\square \\
\square \\
\square \\
\square\end{array}$ & $\begin{array}{l}\square \\
\square \\
\square \\
\square \\
\square \\
\square \\
\square \\
\square\end{array}$ & $\begin{array}{l}\square \\
\square \\
\square \\
\square \\
\square \\
\square \\
\square \\
\square\end{array}$ & $\begin{array}{l}\square \\
\square \\
\square \\
\square \\
\square \\
\square \\
\square \\
\square\end{array}$ & $\begin{array}{l}\square \\
\square \\
\square \\
\square \\
\square \\
\square \\
\square \\
\square\end{array}$ \\
\hline $\begin{array}{l}\text { Bedarfsfeld: Risiko und Absicherung } \\
\text { Forderungsmanagement } \\
\text { Zins- und Währungsmanagement } \\
\text { Sachversicherungen } \\
\text { Lebensversicherungen } \\
\text { Betriebliche Altersvorsorge }\end{array}$ & $\begin{array}{l}\square \\
\square \\
\square \\
\square \\
\square\end{array}$ & $\begin{array}{l}\square \\
\square \\
\square \\
\square \\
\square\end{array}$ & $\begin{array}{l}\square \\
\square \\
\square \\
\square \\
\square\end{array}$ & $\begin{array}{l}\square \\
\square \\
\square \\
\square \\
\square\end{array}$ & $\begin{array}{l}\square \\
\square \\
\square \\
\square \\
\square\end{array}$ & $\begin{array}{l}\square \\
\square \\
\square \\
\square \\
\square\end{array}$ & $\begin{array}{l}\square \\
\square \\
\square \\
\square \\
\square\end{array}$ & $\begin{array}{l}\square \\
\square \\
\square \\
\square \\
\square\end{array}$ & $\begin{array}{l}\square \\
\square \\
\square \\
\square \\
\square\end{array}$ & $\begin{array}{l}\square \\
\square \\
\square \\
\square \\
\square\end{array}$ & $\begin{array}{l}\square \\
\square \\
\square \\
\square \\
\square\end{array}$ & $\begin{array}{l}\square \\
\square \\
\square \\
\square \\
\square\end{array}$ & $\begin{array}{l}\square \\
\square \\
\square \\
\square \\
\square\end{array}$ & $\begin{array}{l}\square \\
\square \\
\square \\
\square \\
\square\end{array}$ & $\begin{array}{l}\square \\
\square \\
\square \\
\square \\
\square\end{array}$ \\
\hline $\begin{array}{l}\text { Bedarfsfeld: Vermögensanlage } \\
\text { Anlagemanagement } \\
\text { Vermögensberatung } \\
\text { Private Vermögensplanung für den Unterneh- } \\
\text { mer }\end{array}$ & $\begin{array}{l}\square \\
\square \\
\square\end{array}$ & $\begin{array}{l}\square \\
\square \\
\square\end{array}$ & $\begin{array}{l}\square \\
\square \\
\square\end{array}$ & $\begin{array}{l}\square \\
\square \\
\square\end{array}$ & $\begin{array}{l}\square \\
\square \\
\square\end{array}$ & $\begin{array}{l}\square \\
\square \\
\square\end{array}$ & $\begin{array}{l}\square \\
\square \\
\square\end{array}$ & $\begin{array}{l}\square \\
\square \\
\square\end{array}$ & $\begin{array}{l}\square \\
\square \\
\square\end{array}$ & $\begin{array}{l}\square \\
\square \\
\square\end{array}$ & $\begin{array}{l}\square \\
\square \\
\square\end{array}$ & $\begin{array}{l}\square \\
\square \\
\square\end{array}$ & $\begin{array}{l}\square \\
\square \\
\square\end{array}$ & $\begin{array}{l}\square \\
\square \\
\square\end{array}$ & $\begin{array}{l}\square \\
\square \\
\square\end{array}$ \\
\hline $\begin{array}{l}\text { Bedarfsfeld: Betriebswirtschaftliche Beratung } \\
\text { Regelmäßiges ganzheitliches Strategiegespräch } \\
\text { Bilanzanalysegespräch } \\
\text { Ratingberatung } \\
\text { Stärken-/ Schwächenanalyse } \\
\text { Risikoanalyse / Risikoprofil } \\
\text { Existenzgründungsberatung } \\
\text { Unternehmensnachfolgeberatung } \\
\text { Projektbeurteilung } \\
\text { Beratung von Auslandsprojekten } \\
\text { Unterstützung bei der } \\
\text { Investitionsplanung }\end{array}$ & $\begin{array}{l}\square \\
\square \\
\square \\
\square \\
\square \\
\square \\
\square \\
\square \\
\square \\
\square\end{array}$ & $\begin{array}{l}\square \\
\square \\
\square \\
\square \\
\square \\
\square \\
\square \\
\square \\
\square \\
\square\end{array}$ & $\begin{array}{l}\square \\
\square \\
\square \\
\square \\
\square \\
\square \\
\square \\
\square \\
\square \\
\square\end{array}$ & $\begin{array}{l}\square \\
\square \\
\square \\
\square \\
\square \\
\square \\
\square \\
\square \\
\square \\
\square\end{array}$ & $\begin{array}{l}\square \\
\square \\
\square \\
\square \\
\square \\
\square \\
\square \\
\square \\
\square \\
\square\end{array}$ & $\begin{array}{l}\square \\
\square \\
\square \\
\square \\
\square \\
\square \\
\square \\
\square \\
\square \\
\square\end{array}$ & $\begin{array}{l}\square \\
\square \\
\square \\
\square \\
\square \\
\square \\
\square \\
\square \\
\square \\
\square\end{array}$ & \begin{tabular}{|l|}
$\square$ \\
$\square$ \\
$\square$ \\
$\square$ \\
$\square$ \\
$\square$ \\
$\square$ \\
$\square$ \\
$\square$ \\
$\square$
\end{tabular} & $\begin{array}{l}\square \\
\square \\
\square \\
\square \\
\square \\
\square \\
\square \\
\square \\
\square \\
\square\end{array}$ & $\begin{array}{l}\square \\
\square \\
\square \\
\square \\
\square \\
\square \\
\square \\
\square \\
\square \\
\square\end{array}$ & $\begin{array}{l}\square \\
\square \\
\square \\
\square \\
\square \\
\square \\
\square \\
\square \\
\square \\
\square\end{array}$ & $\begin{array}{l}\square \\
\square \\
\square \\
\square \\
\square \\
\square \\
\square \\
\square \\
\square \\
\square\end{array}$ & $\begin{array}{l}\square \\
\square \\
\square \\
\square \\
\square \\
\square \\
\square \\
\square \\
\square \\
\square\end{array}$ & $\begin{array}{l}\square \\
\square \\
\square \\
\square \\
\square \\
\square \\
\square \\
\square \\
\square \\
\square\end{array}$ & $\begin{array}{l}\square \\
\square \\
\square \\
\square \\
\square \\
\square \\
\square \\
\square \\
\square \\
\square\end{array}$ \\
\hline $\begin{array}{l}\text { Unterstützung bei der Erstellung einer Planbi- } \\
\text { lanz }\end{array}$ & $\square$ & $\square$ & $\square$ & $\square$ & $\square$ & $\square$ & $\square$ & $\square$ & $\square$ & $\square$ & $\square$ & $\square$ & $\square$ & $\square$ & $\square$ \\
\hline $\begin{array}{l}\text { Unterstützung bei der Liquiditätsplanung } \\
\text { Lohnnebenkostenoptimierung } \\
\text { Workshops / Erfahrungsaustausch zu } \\
\text { betriebswirtschaftlichen Themen }\end{array}$ & $\begin{array}{l}\square \\
\square \\
\square\end{array}$ & $\begin{array}{l}\square \\
\square \\
\square\end{array}$ & $\begin{array}{l}\square \\
\square \\
\square\end{array}$ & $\begin{array}{l}\square \\
\square \\
\square\end{array}$ & $\begin{array}{l}\square \\
\square \\
\square\end{array}$ & $\begin{array}{l}\square \\
\square \\
\square\end{array}$ & $\begin{array}{l}\square \\
\square \\
\square\end{array}$ & $\begin{array}{c}\square \\
\square \\
\square\end{array}$ & $\begin{array}{l}\square \\
\square \\
\square\end{array}$ & $\begin{array}{l}\square \\
\square \\
\square\end{array}$ & $\begin{array}{l}\square \\
\square \\
\square\end{array}$ & $\begin{array}{l}\square \\
\square \\
\square\end{array}$ & $\begin{array}{l}\square \\
\square \\
\square\end{array}$ & $\begin{array}{l}\square \\
\square \\
\square\end{array}$ & $\begin{array}{l}\square \\
\square \\
\square\end{array}$ \\
\hline
\end{tabular}




\section{Anforderungen an eine partnerschaftliche Geschäftsbeziehung}

3.1 Bitte geben Sie zunächst die Wichtigkeit an, die die nachstehenden Merkmale im Rahımen einer partnerschaftlichen Geschäftsbeziehung für Sie haben.

Beurteilen Sie dann, inwieweit Ihre Hausbank die Erwartungen an das jeweilige Kriterium zu Ihrer Zufriedenheit erfültt.

\begin{tabular}{|c|c|c|}
\hline \multirow[b]{3}{*}{ Merkmal } & \multirow{2}{*}{$\begin{array}{c}\text { Wichtigkeit } \\
\begin{array}{c}1=\text { äuBerst unwichtig } \\
7=\text { äuBerst wichtig }\end{array}\end{array}$} & \multirow{2}{*}{$\begin{array}{c}\text { Zufriedenheit } \\
\begin{array}{c}\text { = sehr unzufrieden } \\
7=\text { sehr } \text { zufrieden }\end{array}\end{array}$} \\
\hline & & \\
\hline & $\begin{array}{lllllll}1 & 2 & 3 & 4 & 5 & 6 & 7\end{array}$ & $\begin{array}{|lllllll|}1 & 2 & 3 & 4 & 5 & 6 & 7\end{array}$ \\
\hline Persönlicher Ansprechpartner & $\square \square \square \square \square \square \square$ & पि口वप्व \\
\hline $\begin{array}{l}\text { Konstanz der Bezugsperson } \\
\text { (nach Möglichkeit keine wechselnden } \\
\text { Ansprechpartner) }\end{array}$ & 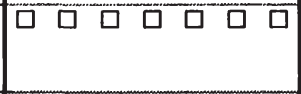 & $\square \square \square \square \square \square \square$ \\
\hline $\begin{array}{l}\text { Ähnliche Einstellungen und Wertvorstellungen } \\
\text { des Betreuers }\end{array}$ & $\square \square \square \square \square \square \square$ & $\square \square \square \square \square \square \square$ \\
\hline Regelınäßiger persönlicher Kontakt & $\square \square \square \square \square \square \square$ & $\square \square \square \square \square \square \square$ \\
\hline Private Kontakte zu Ihrem Firmenkundenbetreuer & 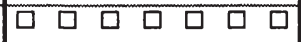 & $\square \square \square \square \square \square \square$ \\
\hline $\begin{array}{l}\text { Betreuer, der auf meine individuelle } \\
\text { Situation eingeht }\end{array}$ & 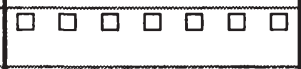 & $\square \square \square \square \square \square \square$ \\
\hline Hinzuziehung von Spezialisten & 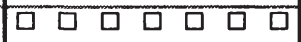 & 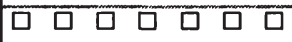 \\
\hline $\begin{array}{l}\text { Bankfachliche Kompetenz des Betreuers (fundierte } \\
\text { bankkaufmännische Ausbildung, gute Produkt- } \\
\text { kenntnis, verständliche Erklärungen) }\end{array}$ & 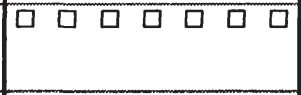 & 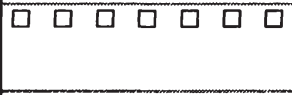 \\
\hline $\begin{array}{l}\text { Betriebswirtschaftliche Kompetenz des Betreuers } \\
\text { (fundierte betriebswirtschaftliche Ausbildung mit } \\
\text { Theorie- und Praxisbezug) }\end{array}$ & पव口पव口व & 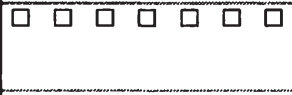 \\
\hline $\begin{array}{l}\text { Aktives Erkennen und Ansprechen von Optimie- } \\
\text { rungspotenzial in Bezug auf die angebotenen Fi- } \\
\text { nanzdienstleistungen }\end{array}$ & 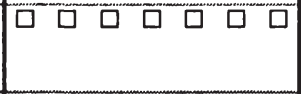 & $\square \square \square \square \square \square \square$ \\
\hline Gegenüberstellung von Alternativen & $\square \square \square \square \square \square \square$ & $\square \square \square \square \square \square \square$ \\
\hline $\begin{array}{l}\text { Aktives Erkennen und Ansprechen von betriebs- } \\
\text { wirtschaftlichen Handlungsfeldern }\end{array}$ & 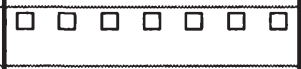 & 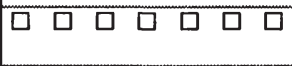 \\
\hline $\begin{array}{l}\text { Regelmäßiges zur Verfügung stellen von Bran- } \\
\text { cheninformationen }\end{array}$ & 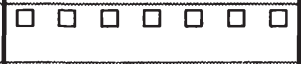 & 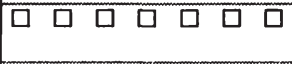 \\
\hline Branchenkenntnis des Betreuers & 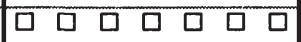 & 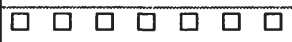 \\
\hline Gegenseitige Vertrauensbereitschaft & पव口पव口व & 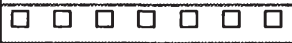 \\
\hline Zuverlässigkeit & $\square \square \square \square \square \square \square$ & $\square \square \square \square \square \square \square$ \\
\hline Termintreue & 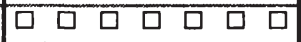 & $\square \square \square \square \square \square \square$ \\
\hline Schnelle Entscheidungen & 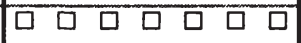 & 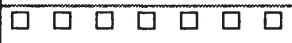 \\
\hline $\begin{array}{l}\text { Offenheit (z.B. offene Ratingkommunikation, } \\
\text { frühzeitige Ansprache von Problemfeldern) }\end{array}$ & $\square \square \square \square \square \square \square$ & $\square \square \square \square \square \square \square$ \\
\hline Transparenz (z.B. bzgl. der Konditionsgestaltung) & 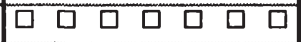 & व $\square \square \square \square \square$ \\
\hline Fairness & $\square \square \square \square \square \square \square$ & 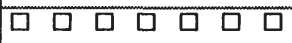 \\
\hline Angenehme Atmosphäre & $\square \square \square \square \square \square \square$ & $\square \square \square \square \square \square \square$ \\
\hline Diskrete Beratungsräume & $\square \square \square \square \square \square \square$ & 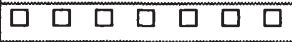 \\
\hline Reratungermine in mein & $\square \square \square \square \square \square \square$ & $\square \square \square \square \square \square \square$ \\
\hline
\end{tabular}

Seite 4 von 6 
3.2 Wie würden Sie aufgrund des Leistungsangebotes und des Erfüllungsgrades der genannten Beziehungsmerkmale die Stärke der Bindung zu Ihrer Hausbank einschätzen?

\begin{tabular}{|c|c|c|c|c|c|c|c|}
\hline & \multicolumn{7}{|c|}{$\begin{array}{c}1 \text { = āußerst schwach ausgeprägt } \\
7=\text { äuBerst stark ausgeprägt }\end{array}$} \\
\hline & 1 & 2 & 3 & 4 & 5 & 6 & 7 \\
\hline Stărke der Bindung zur Hausbank - insgesamt & $\square$ & $\square$ & $\square$ & $\square$ & $\square$ & $\bar{\square}$ & $\bar{\square}$ \\
\hline \multicolumn{8}{|l|}{ Komponenten der Bindung } \\
\hline $\begin{array}{l}\text { Gebundenheit } \\
\text { (Rationale Vorteilhaftigkeit der Geschäftsbeziehung) }\end{array}$ & 口 & $\square$ & $\square$ & 口 & $\square$ & $\square$ & $\square$ \\
\hline $\begin{array}{l}\text { Verbundenheit } \\
\text { (Erfüllung der Anforderungen durch den Firmenkun- } \\
\text { denbetreuer) }\end{array}$ & $\square$ & $\square$ & $\square$ & 口 & $\square$ & $\square$ & $\square$ \\
\hline $\begin{array}{l}\text { Bindung aufgrund des umfangreichen Dienstleistungs- } \\
\text { angebotes }\end{array}$ & $\square$ & $\square$ & $\square$ & $\square$ & $\square$ & $\bar{\square}$ & $\square$ \\
\hline
\end{tabular}

\section{Bedarfsfeld: Betriebswirtschaftliche Beratung}

4.1 Für den Fall, dass Sie im Bedarfsfeld Beratungsdienstleistungen an Stelle Ihrer Hausbank auf Leistungen anderer Kreditinstitute oder Drittdienstleister zurückgreifen hat das welche Gründe (Mehrfachnennungen erlaubt)?

$\square$ Hausbank bietet Dienstleistung nicht an

$\square$ mangelnde betriebswirtschaftliche Kompetenz der Hausbank

$\square$ fehlender Spezialist (Dienstleistung wird dem Firmenkundenbetreuer nicht zugetraut)

$\square$ Sonstige Gründe:

4.2 Bitte geben Sie an, bei welchen Beratungsdienstleistungen aus Ihrer Sicht ein stärkeres Engagement Ihrer Hausbank wünschenswert wäre und kennzeichnen Sie zusătzlich, ob Sie die jeweilige Dienstleistung der Bank aktuell zutrauen oder die Kompetenz in dem jeweiligen Bereich noch erweitert werden sollte.

Markieren Sie bitte auch, ob die Beratungsleistung idealer Weise durch Ihren Firmenkundenbetreuer oder unter Hinzuziehung eines Spezialisten erfolgen sollte.

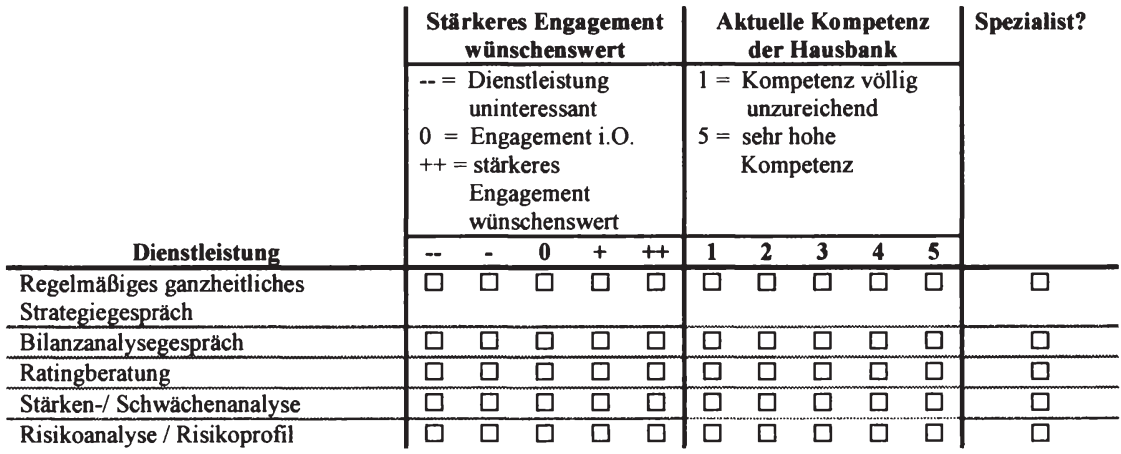

Fortsetzung - Nächste Seite...

Seite 5 von 6 


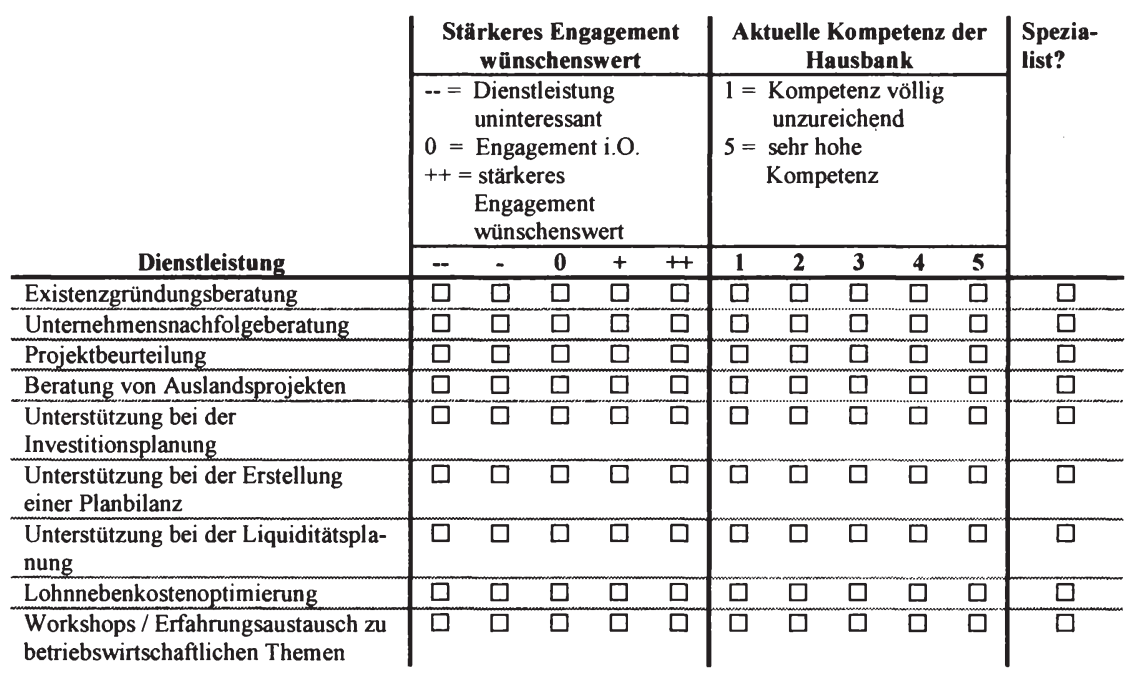

4.3 Bewerten Sie bitte, inwieweit die betriebswirtschaftliche Beratung bei den unterschiedlichen Anbietern ein Ihren Ansprüchen entsprechendes Engagement aufweist und ob Sie die Bepreisung für angemessen halten.

\begin{tabular}{|c|c|c|c|c|c|c|c|c|c|c|}
\hline \multirow[b]{3}{*}{ Dienstleistung } & \multicolumn{5}{|c|}{ Engagement } & \multicolumn{5}{|c|}{ Bepreisung } \\
\hline & \multicolumn{5}{|c|}{$\begin{array}{l}-=\text { = } 8 \text { llig unzureichend } \\
++=\text { sehr hoch }\end{array}$} & \multicolumn{5}{|c|}{$\begin{aligned}--= & \text { viel zu teuer } \\
++ & =\text { ausgezeichnetes Preisleistungs- } \\
& \text { verhältnis }\end{aligned}$} \\
\hline & -- & - & $\mathbf{0}$ & + & ++ & -- & - & $\mathbf{0}$ & + & ++ \\
\hline $\begin{array}{l}\text { Steuerberater } \\
\text { Wirtschaftsprüfer } \\
\text { Unternehmensberater } \\
\text { Hausbank } \\
\text { Zweitkreditinstitut } \\
\text { Finanzdienstleister }\end{array}$ & $\begin{array}{l}\square \\
\square \\
\square \\
\square \\
\square \\
\square\end{array}$ & $\begin{array}{l}\square \\
\square \\
\square \\
\square \\
\square \\
\square\end{array}$ & $\begin{array}{l}\square \\
\square \\
\square \\
\square \\
\square \\
\square\end{array}$ & $\begin{array}{l}\square \\
\square \\
\square \\
\square \\
\square \\
\square \\
\square\end{array}$ & $\begin{array}{l}\square \\
\square \\
\square \\
\square \\
\square \\
\square\end{array}$ & $\begin{array}{l}\square \\
\square \\
\square \\
\square \\
\square \\
\square\end{array}$ & $\begin{array}{l}\square \\
\square \\
\square \\
\square \\
\square \\
\square\end{array}$ & $\begin{array}{l}\square \\
\square \\
\square \\
\square \\
\square \\
\square\end{array}$ & $\begin{array}{l}\square \\
\square \\
\square \\
\square \\
\square \\
\square\end{array}$ & $\begin{array}{l}\square \\
\square \\
\square \\
\square \\
\square \\
\square\end{array}$ \\
\hline
\end{tabular}

4.4 Bitte geben Sie an, inwiefern folgende Aussage auf Sie zutrifft:

„Wenn meine Hausbank bestimmte betriebswirtschaftliche Beratungsdienstleistungen - bei einem spürbaren Mehrwert und zu einem besseren Preisleistungsverhältnis als andere Dienstleister - anbieten würde, würde ich auf das Angebot der Hausbank zurückgreifen.“

\begin{tabular}{c|c|c|c|c|c}
$\begin{array}{c}\text { trifft auf } \\
\text { keinen Fall zu }\end{array}$ & trifft nicht zu & $\begin{array}{c}\text { trifft eher } \\
\text { nicht zu }\end{array}$ & $\begin{array}{c}\text { trifft bedingt } \\
\text { zu }\end{array}$ & trifft zu & $\begin{array}{c}\text { trifft voll- } \\
\text { kommen zu }\end{array}$ \\
\hline$\square$ & $\square$ & $\square$ & $\square$ & $\square$ & $\square$
\end{tabular}


Sind Sie an den Ergebnissen der Befragung interessiert?

Wenn ja, tragen Sie bitte Ihre Daten ein, damit wir Ihnen das Auswertungsergebnis als PDFDokument zusenden können.

Die Adresse wird nicht an Dritte weitergegeben und im Anschluss an den Versand des Ergebnisses gelöscht.

E-Mail:

Firma:

Anrede:

Name:

Vielen Dank für Ihre Teilnahme! 


\section{Rücksendebogen}

\section{Rücksendung bitte an:}

$<$ ABSE $1>$

$<$ ABSE2>

$<$ ABSE3>

$<$ ABSE4>

$<$ ABSE5 $>$

$<$ ABSE6 $>$

\section{Hinweis:}

Den ausgefüllten Fragebogen können Sie auch gern - persönlich oder per Boten - in Ihrer Geschäftsstelle einreichen.

Vielen Dank für Ihre Mithilfe! 
ABSE1:

im November 2006

«ABSE2

‘ABSE3:

ABSE4

ABSE5

ABSE6

AADRZ1

CADRZ2>

(ADRZ3〉

ADRZ4.

ADRZ5:

$\angle A D R Z 6$

\section{Diplomarbeit - Optimlerung des Leistungsangebotes in der Firmenkundenbetreuung}

\section{«ANREDI> \\ ४ANRED2:}

die stetige Verbesserung des Betreuungsangebotes ist ein wesentlicher Anspruch im Firmenkinudengesclıäft der Volks- und Raiffeisenbanken und die Basis einer ganzheitlichen Kundenbetreuung.

Denn nur Banken, die die Auforderungeu ihrer Kunden genau kennen. können Ihr Dienstleistungsportfolio optimal auf die Kundenwünsche abstimmen.

Im Rahmen meines benfsbegleitenden Studiums an der FHDW - Fachhochschule der Wirtschaft möchte ich in meiner Diplomarbeit untersuchen, inwieweit im Firmenkundengeschäft ein Wandel der Anforderungsstruktur gegenüber Banken erkennbar ist.

Vim eine möglichst aussagefähige Studie zu erhalten und damit Verbessertngspotenzial im Betreuungsangebot der Volks- und Raiffeisenbauken aufzudecken, bin ich auf thre Mithilfe angewiesen und möchte Sie herzlich bitten, den beigefïgten Fragebogen auszufüllen oder im Internet an der Befragung - unter nachstehendem Link - teilzunehmen:

Internetlink:

Passwort:

Die Beantwortung des Fragebogens ist. trotz des vermeintlich großen Umfangs, schnell durchzuführen und wird nicht mehr als 15 Minuten Ihrer Zeit beanspruchen.

Vim eine fristgerechte Auswertung zn gewährleisten. bin ich Ihnen für einen Rücklauf bis zum 08 . Dezember 2006 sehr daukbar. Dieser kaun durch Abgabe in Ihrer Geschäftsstelle, Rücksendung oder Interuetteilnahme erfolgen. 
Seite 2 vou 2 zum Schreiben aus November 2006

Selbstverständlich erfolgt die Auswertung anonymisiert. Thre Daten werden vertraulich behandelt und nicht an Dritte weitergegeben.

Die Ergebnisse der Studie stelle ich Ihnen auf Wusch gern zur Verfüging.

Für llure Unterstützung und Duren Beitrag zum Gelingen meiner Diplomarbeit bedanke ich mich schon in Voraus recht herzlich!

Mit freundlichen Grißen,

Nino Raddao 


\section{Anhang 14: Auswertung der empirischen Erhebung}

\begin{tabular}{|l|r|r|}
\hline Sektor & absolut & \multicolumn{1}{|c|}{ relativ } \\
\hline Handel & 33 & $29,46 \%$ \\
\hline Dienstleistung & 31 & $27,68 \%$ \\
\hline Produktion & 29 & $25,89 \%$ \\
\hline Baugewerbe & 16 & $14,29 \%$ \\
\hline Freie Berufe & 3 & $2,68 \%$ \\
\hline Gesamtwert & $\mathbf{1 1 2}$ & $\mathbf{1 0 0 , 0 0 \%}$ \\
\hline
\end{tabular}

Tabelle 14: Sektorzugehörigkeit der Stichprobe

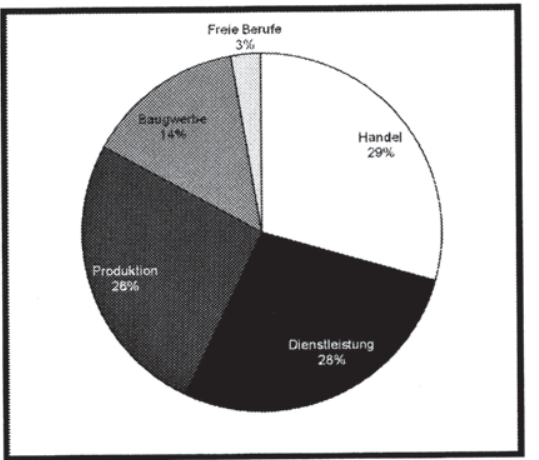

Abbildung 50: Kreisdiagramm: Sektorzugehörigkeit

\begin{tabular}{|l|r|r|}
\hline Eigenkapitalquote & absolut & \multicolumn{1}{c|}{ relativ } \\
\hline unter $10 \%$ & 36 & $33,03 \%$ \\
\hline $10 \%$ bis unter $20 \%$ & 16 & $14,68 \%$ \\
\hline $20 \%$ bis unter $30 \%$ & 27 & $24,77 \%$ \\
\hline über 30\% & 30 & $27,52 \%$ \\
\hline Gesamtwert & $\mathbf{1 0 9}$ & $\mathbf{1 0 0 , 0 0} \%$ \\
\hline
\end{tabular}

Tabelle 15: Eigenkapitalstruktur der Stichprobe

\begin{tabular}{|c|c|c|}
\hline Anzahl aktiver Kontoverbindungen & absolut & relativ \\
\hline 1 & 25 & $22,32 \%$ \\
\hline 2 & 58 & $51,79 \%$ \\
\hline 3 & 22 & $19,64 \%$ \\
\hline 4 & 5 & $4,46 \%$ \\
\hline 5 & 2 & $1,79 \%$ \\
\hline Gesamtwert & 112 & $100,00 \%$ \\
\hline Ø Anzahl aktiver Bankverbindungen & & 2,12 \\
\hline
\end{tabular}

Tabelle 16: Stichprobenstruktur: Anzahl aktiver Kontoverbindungen 


\begin{tabular}{|l|r|r|}
\hline Hausbankstatus & & \\
\hline Volks- und Raiffeisenbanken & 90 & $80,36 \%$ \\
\hline Sparkassen & 17 & $15,18 \%$ \\
\hline Großbanken & 5 & $4,46 \%$ \\
\hline Gesamtwert & $\mathbf{1 1 2}$ & $\mathbf{1 0 0 , 0 0 \%}$ \\
\hline
\end{tabular}

Tabelle 17: Strichprobenstruktur: Banken mit Hausbankstatus

\begin{tabular}{|c|c|c|c|c|c|c|c|c|c|c|c|c|}
\hline \multirow[b]{2}{*}{ Dienstleistung } & \multicolumn{6}{|c|}{ Leistungsangebot } & \multicolumn{6}{|c|}{ Leistungsinanspruchnahme } \\
\hline & 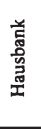 & ) & 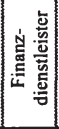 & 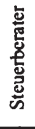 & 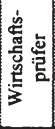 & 岳 & 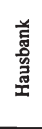 & 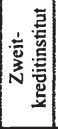 & 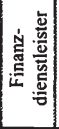 & 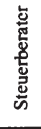 & 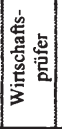 & 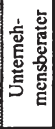 \\
\hline $\begin{array}{l}\text { Bedarfsfeld: Llqulditat und Zahlungsverkehr } \\
\text { Betriebsmittelkredite } \\
\text { Elektronischer Zahlungsverkehr } \\
\text { Cash Management } \\
\text { Auslandszahlungsverkehr }\end{array}$ & $\begin{array}{c}89 \\
100 \\
37 \\
53\end{array}$ & $\begin{array}{l}40 \\
42 \\
19 \\
21\end{array}$ & $\begin{array}{c}11 \\
1 \\
0 \\
0\end{array}$ & $\begin{array}{l}0 \\
1 \\
2 \\
2\end{array}$ & $\begin{array}{l}0 \\
0 \\
0 \\
0\end{array}$ & $\begin{array}{l}4 \\
0 \\
0 \\
1\end{array}$ & $\begin{array}{l}78 \\
84 \\
22 \\
41\end{array}$ & $\begin{array}{l}4 \\
7 \\
5 \\
6\end{array}$ & $\begin{array}{l}1 \\
0 \\
0 \\
0\end{array}$ & $\begin{array}{l}0 \\
1 \\
0 \\
0\end{array}$ & $\begin{array}{l}0 \\
0 \\
0 \\
0\end{array}$ & $\begin{array}{l}0 \\
0 \\
0 \\
0\end{array}$ \\
\hline $\begin{array}{l}\text { Bedarfsfeld: Investition und Finanzierung } \\
\text { Investitionskredite } \\
\text { Vermittlung offentlicher Fordermittel } \\
\text { Hypothekenvermittlung } \\
\text { Immobilienvermittlung } \\
\text { Leasing } \\
\text { Im- und Exportfinanzierung } \\
\text { Bürgschafts- und Garantiegeschaft } \\
\text { Mezzanine Finanzierung }\end{array}$ & $\begin{array}{c}80 \\
54 \\
42 \\
18 \\
39 \\
9 \\
48 \\
6\end{array}$ & $\begin{array}{c}34 \\
18 \\
17 \\
11 \\
17 \\
7 \\
13 \\
4\end{array}$ & $\begin{array}{c}7 \\
7 \\
0 \\
2 \\
23 \\
0 \\
4 \\
1\end{array}$ & $\begin{array}{l}2 \\
1 \\
1 \\
0 \\
0 \\
2 \\
0 \\
2\end{array}$ & $\begin{array}{l}0 \\
0 \\
0 \\
0 \\
0 \\
0 \\
0 \\
0\end{array}$ & $\begin{array}{l}2 \\
7 \\
2 \\
1 \\
1 \\
0 \\
2 \\
3\end{array}$ & $\begin{array}{c}63 \\
41 \\
35 \\
11 \\
22 \\
5 \\
42 \\
1\end{array}$ & $\begin{array}{l}4 \\
4 \\
2 \\
2 \\
3 \\
1 \\
3 \\
1\end{array}$ & $\begin{array}{c}3 \\
0 \\
0 \\
2 \\
19 \\
0 \\
2 \\
0\end{array}$ & $\begin{array}{l}2 \\
0 \\
0 \\
0 \\
0 \\
0 \\
0 \\
0\end{array}$ & $\begin{array}{l}0 \\
0 \\
0 \\
0 \\
0 \\
0 \\
0 \\
0\end{array}$ & $\begin{array}{l}0 \\
5 \\
0 \\
0 \\
0 \\
0 \\
0 \\
0\end{array}$ \\
\hline $\begin{array}{l}\text { Bedarfsfeld: Risilvo und Absicherung } \\
\text { Forderungsmanagement } \\
\text { Zins- und Wahrungsmanagement } \\
\text { Sachversicherungen } \\
\text { Lebensversicherungen } \\
\text { Betriebliche Altersvorsorge }\end{array}$ & $\begin{array}{l}12 \\
12 \\
32 \\
42 \\
42\end{array}$ & $\begin{array}{l}4 \\
11 \\
16 \\
21 \\
21\end{array}$ & $\begin{array}{c}13 \\
2 \\
27 \\
29 \\
33\end{array}$ & $\begin{array}{l}4 \\
0 \\
0 \\
2 \\
2\end{array}$ & $\begin{array}{l}0 \\
0 \\
0 \\
0 \\
0\end{array}$ & $\begin{array}{l}3 \\
2 \\
2 \\
2 \\
3\end{array}$ & $\begin{array}{c}2 \\
8 \\
11 \\
19 \\
14\end{array}$ & $\begin{array}{l}0 \\
4 \\
2 \\
5 \\
3\end{array}$ & $\begin{array}{l}6 \\
0 \\
25 \\
27 \\
26\end{array}$ & $\begin{array}{l}0 \\
0 \\
0 \\
0 \\
0\end{array}$ & $\begin{array}{l}0 \\
0 \\
0 \\
0 \\
0\end{array}$ & $\begin{array}{l}2 \\
2 \\
0 \\
0 \\
1\end{array}$ \\
\hline $\begin{array}{l}\text { Bedarfsfeld: Vermdgensanlage } \\
\text { Anlagemanagement } \\
\text { Vermogensberatung } \\
\text { Private Vermogensplanung für den } \\
\text { Unternehmer }\end{array}$ & $\begin{array}{l}36 \\
30 \\
34\end{array}$ & $\begin{array}{l}18 \\
11 \\
19\end{array}$ & $\begin{array}{c}8 \\
12 \\
14\end{array}$ & $\begin{array}{l}4 \\
6 \\
7\end{array}$ & $\begin{array}{l}0 \\
0 \\
2\end{array}$ & $\begin{array}{l}2 \\
3 \\
4\end{array}$ & $\begin{array}{l}23 \\
15 \\
19\end{array}$ & $\begin{array}{l}3 \\
0 \\
2\end{array}$ & $\begin{array}{l}3 \\
4 \\
5\end{array}$ & $\begin{array}{l}1 \\
5 \\
4\end{array}$ & $\begin{array}{l}0 \\
1 \\
2\end{array}$ & $\begin{array}{l}0 \\
0 \\
0\end{array}$ \\
\hline $\begin{array}{l}\text { Redarfyfeld: Betriebswirtschaftliche Beratung } \\
\text { RegelmaBiges gan/heitliches Strategiegespräch } \\
\text { Bilanzanalysegesprách } \\
\text { Ratingberatung } \\
\text { Stärken-/ Schwächenanalyse } \\
\text { Risikoanalyse / Risikoprofil } \\
\text { Existenzgründungsberatung } \\
\text { Untemehmensnachfolgeberatung } \\
\text { Projektbeurteilung } \\
\text { Beratung von Auslandsprojekten } \\
\text { Unterstützung bei der Investitionsplanung } \\
\text { Unterstützung bei der Erstellung einer } \\
\text { Planbilanz } \\
\text { Unterstützung bei der Liquiditatsplanung } \\
\text { Lohnnebenkostenoptimierung } \\
\text { Workshops / Erfahrungsaustausch zu betriebs- } \\
\text { wirtschaftlichen Themen }\end{array}$ & $\begin{array}{c}43 \\
69 \\
54 \\
19 \\
12 \\
20 \\
4 \\
3 \\
4 \\
16 \\
6 \\
13 \\
4 \\
13\end{array}$ & $\begin{array}{c}19 \\
22 \\
22 \\
5 \\
2 \\
6 \\
1 \\
1 \\
1 \\
5 \\
0 \\
2 \\
1 \\
6\end{array}$ & $\begin{array}{l}2 \\
3 \\
3 \\
1 \\
2 \\
0 \\
0 \\
0 \\
0 \\
0 \\
1 \\
1 \\
3 \\
1\end{array}$ & $\begin{array}{c}18 \\
29 \\
5 \\
7 \\
7 \\
5 \\
18 \\
2 \\
0 \\
7 \\
9 \\
8 \\
7 \\
8\end{array}$ & $\begin{array}{l}4 \\
7 \\
1 \\
2 \\
2 \\
1 \\
5 \\
0 \\
0 \\
4 \\
1 \\
0 \\
0 \\
1\end{array}$ & $\begin{array}{c}9 \\
4 \\
3 \\
10 \\
7 \\
2 \\
3 \\
7 \\
5 \\
8 \\
9 \\
10 \\
7 \\
5\end{array}$ & $\begin{array}{c}34 \\
49 \\
42 \\
17 \\
11 \\
15 \\
3 \\
4 \\
1 \\
11 \\
3 \\
10 \\
2 \\
6\end{array}$ & $\begin{array}{l}0 \\
2 \\
2 \\
0 \\
0 \\
1 \\
0 \\
0 \\
2 \\
0 \\
0 \\
0 \\
0 \\
0 \\
1\end{array}$ & $\begin{array}{l}1 \\
1 \\
1 \\
0 \\
0 \\
0 \\
0 \\
0 \\
0 \\
0 \\
0 \\
0 \\
1 \\
0\end{array}$ & $\begin{array}{c}16 \\
23 \\
4 \\
5 \\
5 \\
1 \\
14 \\
1 \\
0 \\
13 \\
9 \\
7 \\
6 \\
5\end{array}$ & $\begin{array}{l}2 \\
6 \\
1 \\
1 \\
2 \\
1 \\
3 \\
0 \\
0 \\
1 \\
2 \\
1 \\
0 \\
1\end{array}$ & $\begin{array}{l}6 \\
3 \\
1 \\
6 \\
5 \\
3 \\
2 \\
5 \\
2 \\
4 \\
5 \\
7 \\
3 \\
4\end{array}$ \\
\hline
\end{tabular}

Tabelle 18: Leistungsangebot und -inanspruchnahme bei verschiedenen Dienstleistern 


\begin{tabular}{|c|c|c|}
\hline \multicolumn{3}{|c|}{$\begin{array}{l}\text { Hypothesenschema zur Uberprüfung einer potenziellen Basisanforderung } \\
\text { mittels Importance Grid }\end{array}$} \\
\hline 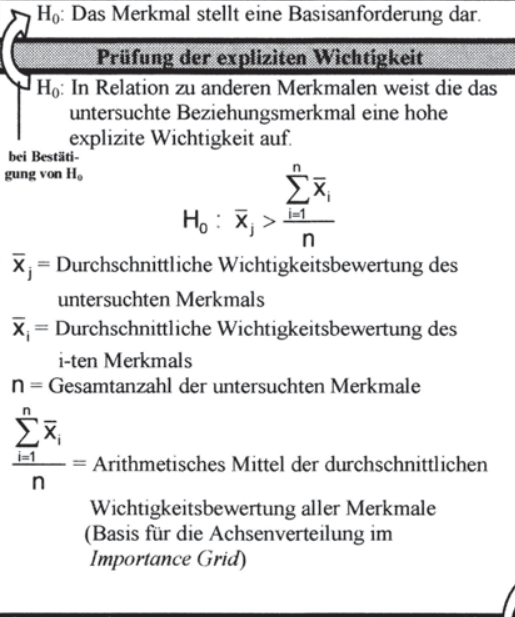 & 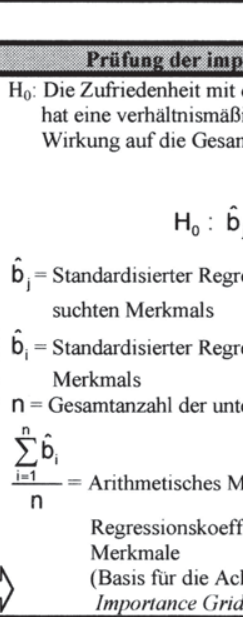 & $\begin{array}{l}\text { ziten Wichtigkeit } \\
\text { em jeweiligen Merkmal } \\
\text { eher geringe, positive } \\
\text { zufriedenheit. } \\
\text { sei } \\
\text { sionskoeftäti- } \\
\text { sionskoeffizient des i-ten } \\
\text { suchten Merkmale } \\
\text { sel der standardisierten } \\
\text { tienten aller } \\
\text { enverteilung im }\end{array}$ \\
\hline $\begin{array}{l}\text { bei Ablehnung } \\
\text { von } \mathrm{H}_{0}\end{array}$ & $\begin{array}{l}\begin{array}{l}\text { Prüfung der Regressions- } \\
\text { funktion } \\
\mathrm{H}_{0} \text { : Die Regressionsfunktion } \\
\text { weist keinen signifi- } \\
\text { kanten Zusammenhang, } \\
\text { bei } \alpha=0,10 \text { auf } \\
\mathrm{H}_{0}: \beta_{1}=\beta_{2} \ldots=\beta_{\mathrm{j}}=0 \\
\beta=\text { wahrer Regressions- } \\
\quad \text { koeffizient } \\
\rightarrow \text { F-Statistik: } \mathrm{H}_{0}: \mathrm{F}_{\mathrm{cmp}} \leq \mathrm{F}_{\mathrm{tab}}\end{array}\end{array}$ & 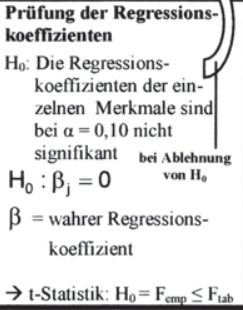 \\
\hline
\end{tabular}

Abbildung 51: Hypothesenschema zur Überprüfung von Basisanforderungen 


\begin{tabular}{|c|c|c|}
\hline \multicolumn{3}{|c|}{$\begin{array}{l}\text { Hypothesenschema zur Überprüfung einer potenziellen (wichtigen) Leistungsanforderung } \\
\text { mittels Importance Grid }\end{array}$} \\
\hline \multicolumn{3}{|l|}{ 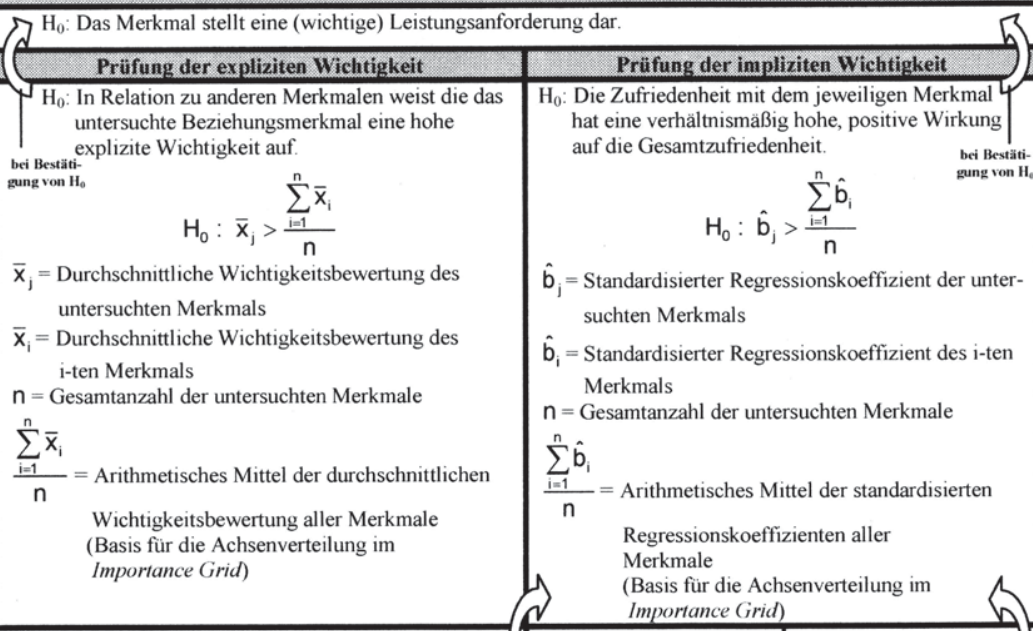 } \\
\hline bei $A$ & $\begin{array}{l}\begin{array}{l}\text { Prüfung der Regressions- } \\
\text { funktion } \\
J_{\mathrm{H}_{0}} \text { : Die Regressionsfunktion } \\
\text { weist keinen signifi- } \\
\text { kanten Zusammenhang, } \\
\text { bei } \alpha=0,10 \text { auf } \\
\mathrm{H}_{0}: \beta_{1}=\beta_{2} \ldots=\beta_{\mathrm{j}}=0 \\
\beta=\text { wahrer Regressions- } \\
\quad \text { koeffizient } \\
\rightarrow \text { F-Statistik: } \mathrm{H}_{0}: \mathrm{F}_{\text {cmp }} \leq \mathrm{F}_{\mathrm{tab}}\end{array}\end{array}$ & 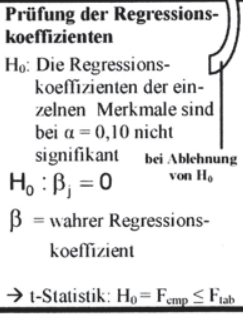 \\
\hline
\end{tabular}

Abbildung 52: Hypothesenschema zur Überprüfung von (wichtigen) Leistungsanforderungen 


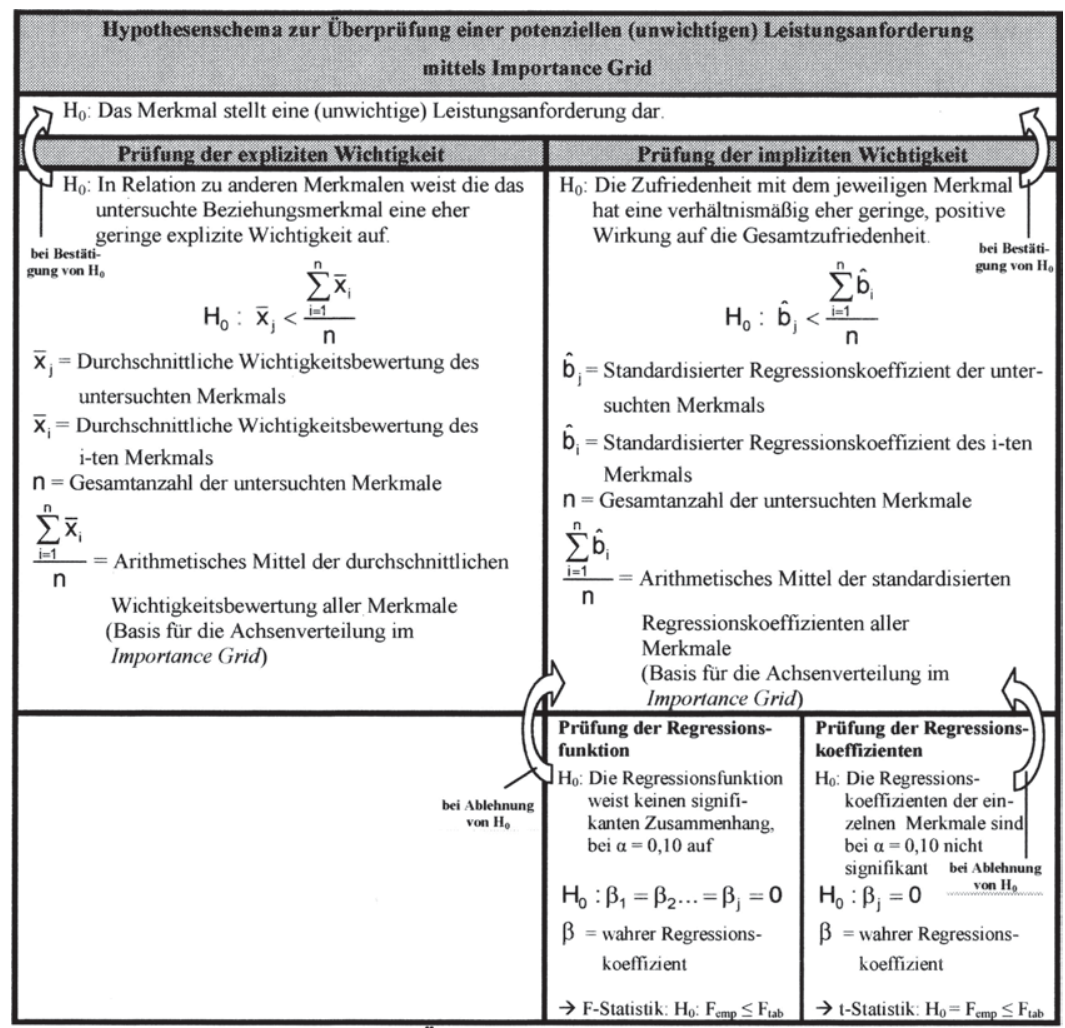

Abbildung 53: Hypothesenschema zur Überprüfung von (unwichtigen) Leistungsanforderungen 


\begin{tabular}{|c|c|c|}
\hline \multicolumn{3}{|c|}{$\begin{array}{l}\text { Hypothesenschema zur Überprïfung einer potenziellen Begeisterungsanforderung } \\
\text { mittels Importance Grid }\end{array}$} \\
\hline \multirow[b]{2}{*}{ 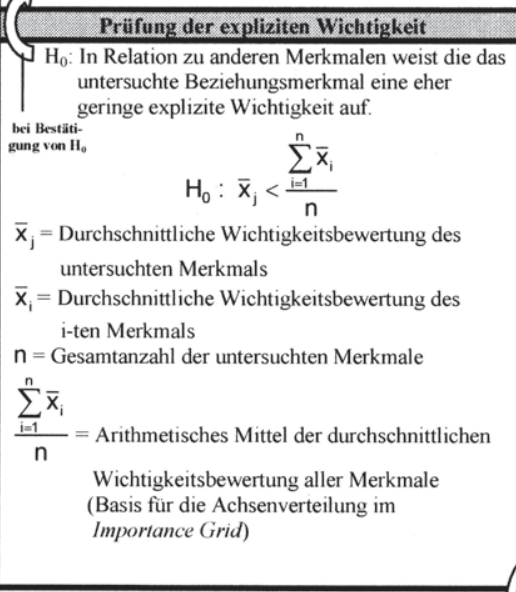 } & \multicolumn{2}{|c|}{ Prüfung der impliziten Wichtigkeit } \\
\hline & $\begin{array}{l}\mathrm{H}_{0} \text { : Die Zufriedenheit mit } \\
\text { hat eine verhältnismäß } \\
\text { auf die Gesamtzufried } \\
\qquad \mathrm{H}_{0}: \hat{\mathrm{b}} \\
\hat{\mathrm{b}}_{\mathrm{j}}=\text { Standardisierter Regr } \\
\text { suchten Merkmals } \\
\hat{\mathrm{b}}_{\mathrm{i}}=\text { Standardisierter Regr } \\
\quad \text { Merkmals } \\
\mathrm{n}=\text { Gesamtanzahl der unt } \\
\sum_{\mathrm{i}=1}^{\mathrm{n}} \hat{\mathrm{b}}_{\mathrm{i}} \\
\mathrm{n}\end{array}$ & $\begin{array}{l}\text { em jeweiligen Merkmal } \\
\text { hohe, positive Wirkung } \\
\text { heit. } \\
\text { ssionskoeffizient des i-ten } \\
\text { ssionskoeffizient der unter- } \\
\text { sei Bestäti } \\
\text { gung von II }\end{array}$ \\
\hline-5 & $\begin{array}{l}\text { Prüfung der Regressions- } \\
\text { funktion } \\
\mathrm{H}_{0} \text { : Die Regressionsfunktion } \\
\text { weist keinen signifi- } \\
\text { kanten } \text { Zusammenhang, } \\
\text { bei } \alpha=0,10 \text { auf } \\
\mathrm{H}_{0}: \beta_{1}=\beta_{2} \ldots=\beta_{\mathrm{j}}=0 \\
\beta=\text { wahrer Regressions- } \\
\quad \text { koeffizient } \\
\rightarrow \text { F-Statistik: } \mathrm{H}_{0}: \mathrm{F}_{\text {emp }} \leq \mathrm{F}_{\mathrm{tab}}\end{array}$ & 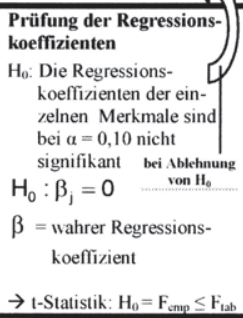 \\
\hline
\end{tabular}

Abbildung 54: Hypothesenschema zur Überprüfung von Begeisterungsanforderungen 


\begin{tabular}{|c|c|c|c|c|c|c|c|}
\hline & & $\begin{array}{l}\text { Nachtolgeb } \\
\text { eratung (E.) }\end{array}$ & $\begin{array}{l}\text { Nachfolgeb } \\
\text { ergtung (K) }\end{array}$ & $\begin{array}{c}\text { Imvestitionspl } \\
\text { anung }(E .)\end{array}$ & 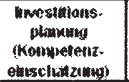 & $\begin{array}{l}\text { Workshops } \\
\text { (E.) }\end{array}$ & $\begin{array}{l}\text { Workshops } \\
\text { (K) }\end{array}$ \\
\hline Nachfolgeberatung (E) & $\begin{array}{l}\text { Pearson Conglation } \\
\text { Sig. (2-talled) } \\
N\end{array}$ & $\begin{array}{r}1,000 \\
99\end{array}$ & $\begin{array}{r}.149 \\
.168 \\
87\end{array}$ & $\begin{array}{r}.224 \\
.027 \\
98\end{array}$ & $\begin{array}{r}114 \\
.098 \\
92\end{array}$ & $\begin{array}{r}.407 \\
.000 \\
96\end{array}$ & $\begin{array}{r}.085 \\
.433 \\
88\end{array}$ \\
\hline Nachfolgeberatung (K) & $\begin{array}{l}\text { Pearson Corrolation } \\
\text { Sig. (2-talled) } \\
\mathrm{N}\end{array}$ & $\begin{array}{r}.149 \\
.168 \\
87 \\
\end{array}$ & $\begin{array}{r}1,000 \\
87 \\
\end{array}$ & $\begin{array}{r}.247^{*} \\
.022 \\
86 \\
\end{array}$ & $\begin{array}{r}.533 \\
.000 \\
84\end{array}$ & $\begin{array}{r}-.034 \\
.760 \\
85 \\
\end{array}$ & $\begin{array}{r}.652^{\circ} \\
.000 \\
83 \\
\end{array}$ \\
\hline 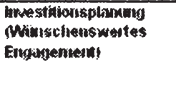 & $\begin{array}{l}\text { Pearson Correlation } \\
\text { Sig. (2-lailed) } \\
N\end{array}$ & $\begin{array}{l}.224^{\circ} \\
.027 \\
98\end{array}$ & $\begin{array}{r}.247^{*} \\
.022 \\
86\end{array}$ & $\begin{array}{r}1,000 \\
. \\
99\end{array}$ & \begin{tabular}{c|}
.312 \\
.002 \\
93
\end{tabular} & $\begin{array}{r}.435 \\
.000 \\
96\end{array}$ & $\begin{array}{r}.158 \\
.141 \\
88\end{array}$ \\
\hline Imvestitionsplanung (K) & $\begin{array}{l}\text { Pearson Correlation } \\
\text { Sig. (2-tailed) } \\
\mathrm{N}\end{array}$ & $\begin{array}{r}.174 \\
.098 \\
92 \\
\end{array}$ & $\begin{array}{r}.533^{-4} \\
.000 \\
84\end{array}$ & $\begin{array}{r}.312 \\
.002 \\
93\end{array}$ & $\begin{array}{r}1,000 \\
93\end{array}$ & $\begin{array}{r}.205 \\
.053 \\
90\end{array}$ & $\begin{array}{r}.646^{-} \\
.000 \\
87\end{array}$ \\
\hline Workshops (E.) & $\begin{array}{l}\text { Pearson Correlation } \\
\text { Sig. (2-1ailed) } \\
N\end{array}$ & $\begin{array}{r}.407 \\
.000 \\
96\end{array}$ & $\begin{array}{r}-.034 \\
.760 \\
85\end{array}$ & $\begin{array}{r}.435^{-} \\
.000 \\
96\end{array}$ & $\begin{array}{r}.205 \\
.053 \\
90 \\
\end{array}$ & $\begin{array}{r}1,000 \\
96\end{array}$ & $\begin{array}{r}.031 \\
.778 \\
87 \\
\end{array}$ \\
\hline Workshops (K) & $\begin{array}{l}\text { Pearson Corrolation } \\
\text { Sig. (2-tailed) } \\
\text { N }\end{array}$ & $\begin{array}{r}.085 \\
.433 \\
08\end{array}$ & $\begin{array}{r}.652 \\
.000 \\
83\end{array}$ & $\begin{array}{r}.158 \\
.141 \\
88 \\
\end{array}$ & $\begin{array}{r}.646^{\prime} \\
.000 \\
81\end{array}$ & $\begin{array}{r}.031 \\
.778 \\
87\end{array}$ & $\begin{array}{r}1,000 \\
88\end{array}$ \\
\hline
\end{tabular}

Tabelle 19: Korrelationsanalyse: Betriebswirtschaftliches Serviceangebot 


\section{Literaturverzeichnis}

Bailom, Franz / Hinterhuber, Hans H. / Matzler, Kurt / Sauerwein, EImar (1996): Das Kano-Modell der Kundenzufriedenheit, in: Marketing ZFP, 1996, Heft 2,

S. $117-126$

Bailom, Franz / Matzler, Kurt (2006):

Messung von Kundenzufriedenheit, in: Hinterhuber, Hans H. / Matzler, Kurt (Hrsg.) (2006), Kundenorientierte Unternehmensführung, 5. Auflage, Wiesbaden 2006, S. 241-270

Balz, Ulrich / Bordemann, Heinz-Gerd (2004):

Mehr miteinander reden, in: Bankinformation, 2004, Heft 3, S. 21-24

Bastian, Nicole / Müller, Oliver (2005):

Deutsche Bank geht auf Mittelstand zu, in: Handelsblatt, 04.01.2005, S. 17

Bliemel, Friedhelm / Kotler, Philip (2005):

Marketing-Management - Analyse, Planung und Verwirklichung, 10. Auflage, München 2005

BMS Consulting GmbH (2006):

MinD.banker Produktbeschreibung, Düsseldorf 2006

Bookhagen, Bettina (2006):

Erhebliche Identifikationsfunktion - „Wir machen den Weg frei“" groß inszeniert, in: Bankinformation, 2006, Heft 6, S. 56-59

Börner, Christoph J. (2005):

Konzeptioneller Rahmen: Strategisches Management und Strategieparameter, in: Börner, Christoph J. / Maser, Harald / Schulz, Thomas Christian (Hrsg.) (2005), Bankstrategien im Firmenkundengeschäft, Wiesbaden 2005, S. 31-63

Braunstein, Christine / Herrmann, Andreas / Huber, Frank (2006):

Der Zusammenhang zwischen Produktqualität, Kundenzufriedenheit und Unternehmenserfolg, in: Hinterhuber, Hans H. / Matzler, Kurt (Hrsg.) (2006), Kundenorientierte Unternehmensführung, 5. Auflage, Wiesbaden 2006, S. 67-83

Brenken, Anke (2006):

KfW-Studie: Die Globalisierung des Mittelstandes: Chancen und Risiken, Frankfurt a.M. 2006

\section{Bruhn, Manfred (2003):}

Kundenorientierung: Bausteine für ein exzellentes Customer Relationship Management, 2. Auflage, München 2003 
Bruhn, Manfred / Homburg, Christian (2005):

Kundenbindungsmanagement - Eine Einführung in die theoretischen und praktischen Problemstellungen, in: Bruhn, Manfred / Homburg, Christian (Hrsg.) (2005), Handbuch Kundenbindungsmanagement, 5. Auflage, Wiesbaden 2005, S. 3-37

Bruhn, Manfred / Meffert, Heribert (2006):

Dienstleistungsmarketing: Grundlagen - Konzepte - Methoden, 5. Auflage, Wiesbaden 2006

\section{Bufka, Jürgen / Eichelmann, Thomas (2002):}

Erhöhung der Kundenzufriedenheit im Firmenkundengeschäft durch bedarfsorientierte Segmentierung - Ergebnisse einer empirischen Studie, in: Achenbacher, Wieland / Steffens, Udo (Hrsg.) (2002), Strategisches Management in Banken, Frankfurt a.M. 2002, S. 123-138

\section{Bundesverband der deutschen Volksbanken- und Raiffeisenbanken e.V.} - BVR (2001):

Bündelung der Kräfte: Die gemeinsame Strategie - Abschlussbericht

Bundesverband der deutschen Volksbanken- und Raiffeisenbanken e.V. - BVR (2004):

Kundensegmentierung für das Firmenkundengeschäft - Leitfaden

Bundesverband der deutschen Volksbanken- und Raiffeisenbanken e.V. - BVR (2006a):

Finanzverbund: Genossenschaftsbanken, http://www.bvr.de/public.nsf/index.html!ReadForm\&main=3\&sub=20,

Stand: 21.11.2006

Bundesverband der deutschen Volksbanken- und Raiffeisenbanken e.V. - BVR (2006b):

Finanzverbund: Genossenschaften, https://www.bvr.de/public.nsf/index.html!ReadForm\&main=3\&sub=50, Stand: 21.11.2006

Bundesverband der deutschen Volksbanken- und Raiffeisenbanken e.V. - BVR (2006c):

Finanzverbund: Verbundpartner, https://www.bvr.de/public.nsf/index.html!ReadForm\&main=3\&sub=30, Stand: 21.11.2006 


\section{Bundesverband der deutschen Volksbanken- und Raiffeisenbanken e.V.} - BVR (2006d):

Verband: Aufgaben, https://www.bvr.de/public.nsf/index.html?ReadForm\&main=4\&sub=10, Stand: 21.11 .2006

Bundesverband der deutschen Volksbanken- und Raiffeisenbanken e.V. - BVR (2006e):

VR-Finanzplan Mittelstand - Projekthandbuch

Bundesverband der deutschen Volksbanken- und Raiffeisenbanken e.V. - BVR (2006f):

VR-Finanzplan Mittelstand - CD-ROM zum Projekthandbuch

Burt, Ronald S. / Camerer, Colin F. / Rousseau, Denise M. / Sitkin, Sim B. (1998): Not so different at all: A cross-discipline view of trust, in: Academy of Management Review, 1998, Heft 3, S. 393-404

Büschgen, Hans E. (2000):

Strategische Positionierung und Profilierung der deutschen Sparkassen als regionale Finanzdienstleister im Euro-Land, in: Zeitschrift für das gesamte Kreditwesen, 2000, Heft 11, S. 580-595

Büschgen, Anja / Büschgen, Hans E. (2002):

Bankmarketing, 2. Auflage, Düsseldorf 2002

Desphandé, Rohit / Moormann, Christine / Zaltman, Gerald (1992):

Relationships between Providers and Users of Market Research: The Dynamics of Trust within and Between Organizations, in: Journal of Marketing Research, 1992, Heft 8, S. 314-328

Deutscher Sparkassen- und Giroverband - DSGV (2006a):

Organisation: Sparkassen, http://www.dsgv.de/de/sparkassenfinanzgruppe/organisation/sparkassen/index.html, Stand: 21.11.2006

\section{Deutscher Sparkassen- und Giroverband - DSGV (2006b):}

Organisation: Landesbanken, http://www.dsgv.de/de/sparkassenfinanzgruppe/organisation/landesbanken/index.html, Stand: 21.11 .2006

\section{Diller, Hermann (1996):}

Kundenbindung als Marketingziel, in: Marketing ZFP, 1996, Heft 2, S. 81-94 
von den Eichen, Stephan A. Friedrich/ Hinterhuber, Hans H. / Matzler, Kurt / Stahl, Heinz K. (2006):

Kundenzufriedenheit und Kundenwert, in: Hinterhuber, Hans H. / Matzler, Kurt (Hrsg.) (2006), Kundenorientierte Unternehmensführung, 5. Auflage, Wiesbaden 2006, S. 222-239

Elsas, Ralf (2001):

Die Bedeutung der Hausbank, Wiesbaden 2001

Färber, Bernd / Hopfner, Wilfried (2006):

Vertriebsintensivierung - Privatkundenstrategie 2012, in: Effert, Detlef / Hanreich, Wilfried (Hrsg.) (2006), Ganzheitliche Beratung bei Banken, Wiesbaden 2006, S. 33-53

FaBnacht, Martin / Homburg, Christian (2001):

Kundennähe, Kundenzufriedenheit und Kundenbindung bei Dienstleistungsunternehmen, in: Bruhn, Manfred / Meffert, Heribert (Hrsg.) (2001), Handbuch Dienstleistungsmanagement, 2. Auflage, Wiesbaden 2001, S. 441-464

Fortis Bank (2006):

Home: Unsere Lösungen: Ihre Forderungen effektiv verwalten: Verringern Sie die Verwaltungsauslastung, http://www.fortisbusiness.com/fbweb/deu_de/content/skills/skills_receivables_worklo ad standard de.html,

Stand: 11.12 .2006

Georgi, Dominik (2005):

Kundenbindungsmanagement im Kundenlebenszyklus in: Bruhn, Manfred / Homburg, Christian (Hrsg.) (2005), Handbuch Kundenbindungsmanagement, 5. Auflage, Wiesbaden 2005, S. 229-249

Grönroos, Christian (2000):

Service management and marketing: a customer relationship management approach, 2. Auflage, Chichester 2000

Günterberg, Brigitte / Wolter, Hans-Jürgen (2002):

Unternehmensgrößenstatistik 2001/2002 - Daten und Fakten, Institut für Mittelstandsforschung Bonn, Bonn 2002, S. 1-22

Hartmann-Wendels, Thomas / Pfingsten, Andreas / Weber, Martin (2007): Bankbetriebslehre, 4. Auflage, Berlin 2007 
Hinterhuber, Hans H. / Matzler, Kurt / Stahl, Heinz K. (2006):

Die Customer-based View der Unternehmung, in: Hinterhuber, Hans H. /

Matzler, Kurt (Hrsg.) (2006), Kundenorientierte Unternehmensführung, 5. Auflage, Wiesbaden 2006, S. 4-31

Hippmann, Hans-Dieter (2003):

Statistik, 3. Auflage, Stuttgart 2003

Holböck, Josef (2006):

Wie profitabel ist die ganzheitliche Finanzberatung?, in: Effert, Detlef / Hanreich, Wilfried (Hrsg.) (2006), Ganzheitliche Beratung bei Banken, Wiesbaden 2006, S. $169-199$

Hunt, Shelby D. / Morgan, Robert M. (1994):

The Commitment-Trust Theory of Relationship Marketing, in: Journal of Marketing, 1994, Heft 7, S. 20-38

Institut für Mittelstandsforschung Bonn - IfM Bonn (2006):

Statistik: Definitionen und Schlüsselzahlen des Mittelstands in Deutschland, http://www.ifm-bonn.org/index.htm?/dienste/definition.htm,

Stand: 25.11 .2006

Käser, Burkhard / Putzer, Annette / Rinker, Andreas (2004):

zeb/-Firmenkundenstudie 2004, Münster 2004

Klingbeil, Ernst / Yousefian, Patrick (2002):

Bundesweites Pilotprojekt für das BVR-II-Rating, in: Bankinformation, 2002, Heft 2, S. $28-29$

Köhler, Peter / Potthoff Christian (2005):

Commerzbank umwirbt Mittelstand, in: Handelsblatt, 05.12.2005, S. 29

Köhler, Richard (2005):

Kundenorientiertes Rechnungswesen, in: Bruhn, Manfred / Homburg, Christian (Hrsg.) (2005), Handbuch Kundenbindungsmanagement, 5. Auflage, Wiesbaden 2005, S. 401-433

Krafft, Manfred (2007):

Kundenbindung und Kundenwert, 2. Auflage, Heidelberg 2007

KrauB, Carsten (2006):

Fit für die Zukunft - Erfolgreiche Restrukturierung des Firmenkundengeschäfts, ADG Akademie deutscher Genossenschaften - Seminarbeitrag, Montabaur 2006 
Kroeber-Riel, Werner / Weinberg, Peter (2003):

Konsumentenverhalten, 8. Auflage, München 2003

Krüger, Markus (2002):

Gestaltung der Zukunft kommt voran, in: Bankinformation, 2002, Heft 6, S. 6-15

Lambert, Martin (2002):

Sparkassen-Finanzgruppe: Berater und Finanzier des deutschen Mittelstands, in:

Sparkasse 119, 2002, Heft 3, S. 104-107

Luhmann, Niklas (2000):

Vertrauen. Ein Mechanismus der Reduktion sozialer Komplexität, 4. Auflage,

Stuttgart 2000

Matzler, Kurt / Stahl, Heinz K. (2000):

Kundenzufriedenheit und Unternehmenswertsteigerung, in: Die Betriebswirtschaft, 2000, Heft 5, S. 626-640

Matzler, Kurt / Sauerwein, Elmar (2002):

The Factor Structure of Customer Satisfaction: An empirical Test of the Importance Grid and the Penalty-Reward-Contrast Analysis, in: International Journal of Service Industry Management, 2002, Heft 4, S. 314-332

Matzler, Kurt / Sauerwein, Elmar / Stark, Christian (2006):

Methoden zur Identifikation von Basis-, Leistungs- und Begeisterungsfaktoren, in: Hinterhuber, Hans H. / Matzler, Kurt (Hrsg.) (2006), Kundenorientierte Unternehmensführung, 5. Auflage, Wiesbaden 2006, S. 290-313

Meffert, Heribert (2000):

Marketing - Grundlagen marktorientierter Unternehmensführung, 9. Auflage, Wiesbaden 2000

Meffert, Heribert (2005):

Kundenbindung als Element moderner Wettbewerbsstrategien, in: Bruhn, Manfred / Homburg, Christian (Hrsg.) (2005), Handbuch Kundenbindungsmanagement, 5. Auflage, Wiesbaden 2005, S. 145-166

\section{Neuhaus, Patricia / Stauss, Bernd (2006):}

Das Qualitative Zufriedenheitsmodell (QZM), in: Hinterhuber, Hans H. /

Matzler, Kurt (Hrsg.) (2006), Kundenorientierte Unternehmensführung, 5. Auflage, Wiesbaden 2006, S. 81-96

Nowak, Helge (2002):

Ratings für alle Segmente, in: Bankinformation, 2002, Heft 5, S. $22-29$ 
o.V. (2005):

RZB wirbt um deutsche Firmen: Mittelstand im Visier - Osteuropa-Netzwerk als Alleinstellungsmerkmal, in: Börsen-Zeitung, 2005, Heft 2007, S. 4

\section{Paul, Stephan (2002):}

Umbrüche im Finanzsystem - veränderte Rahmenbedingungen für das Private Banking, in: Carl, Reinhard / Letzing, Marc (Hrsg.) (2002), Finanzberatung - Persönlichkeit und Know-how für die umfassende Finanzberatung, Stuttgart 2002, S. 21-39

Porter, Michael E. (1999):

Wettbewerbsstrategie - Methoden zur Analyse von Branchen und Konkurrenten, 10. Auflage, Frankfurt a.M. / New York 1999

Porter, Michael E. (2000):

Wettbewerbsvorteile - Spitzenleistungen erreichen und behaupten, 6. Auflage, Frankfurt a.M. 1999

\section{Sauerwein, Elmar (2000):}

Das Kano-Modell der Kundenzufriedenheit - Realibität und Validität einer Methode zur Klassifizierung von Produkteigenschaften, Dissertation, Wiesbaden 2000

\section{Schieble, Michael / Vater, Dirk / Wisskirchen, Cornel (2005):}

Bain \& Company Germany and Switzerland Inc. - Kundenorientierte Wachstumsstrategien, München 2005

\section{Schierenbeck, Henner (2001):}

Relationship Management als zentraler Erfolgsfaktor im Firmenkundengeschäft, in: Müller, Stephan / Rolfes, Bernd / Schierenbeck, Henner (Hrsg.) (2001), Das Firmenkundengeschäft - ein Wertevernichter?, Frankfurt a.M. 2001, S. 187-216

\section{Schlosser, Christoph (2004):}

In der Oper gibt es keine Volksmusik, in: Bankinformation, 2004, Heft 7, S. 28-31

\section{Schmidt, Thomas (2001):}

Die neue Rolle des Firmenkundenbetreuers im mittelständischen Firmenkundengeschäft, Aachen 2001

\section{Schmoll, Anton (2006):}

Vertriebsoptimierung im Firmenkundengeschäft, Wien 2006

\section{Schröder, Gustav Adolf (2001):}

Portfolio-Analyse im Kundengeschäft, in: Rolfes, Bernd / Schierenbeck, Henner / Schüller, Stephan (2001), Handbuch Bankcontrolling, Wiesbaden 2001, S. 595-606 
Schulz, Thomas Christian (2005):

Strategische Segmentbildung im Firmenkundengeschäft der Banken, in:

Börner, Christoph J. / Maser, Harald / Schulz, Thomas Christian (Hrsg.) (2005),

Bankstrategien im Firmenkundengeschäft, Wiesbaden 2005, S. 66-92

Segbers, Klaus (2007):

Die Geschäftsbeziehung zwischen mittelständischen Unternehmen und ihrer

Hausbank, Dissertation, Westfälische Wilhelms-Universität Münster 2006, Frankfurt am Main 2007

Söllner, Albrecht (1999):

Asymmetrical Commitment in Business Relationships, in: Journal of Business

Research, 1999, Heft 11, S. 219-233

Stahl, Heinz K. (2006):

Kundenloyalität kritisch betrachtet, in: Hinterhuber, Hans H. / Matzler, Kurt (Hrsg.) (2006), Kundenorientierte Unternehmensführung, 5. Auflage, Wiesbaden 2006, S. 4-31

Straub, Marc-R. (2006):

Erfolgsfaktoren von Banken im Firmenkundengeschäft, Dissertation, Universität Düsseldorf 2005, Wiesbaden 2006

Steffens, Udo (2002):

Chancen und Risiken der deutschen Banking \& Finance-Branche - eine strategische Analyse, in: Achenbacher, Wieland / Steffens, Udo (Hrsg.) (2002), Strategisches Management in Banken, Frankfurt a.M. 2002, S. 79-103

Taistra, Gregor (2003):

Basel II - aktueller Stand und Auswirkungen auf die Mittelstandsfinanzierung, KfW Arbeitspapier, 2003, http://www.kfw.de/DE_Home/Research/Sonderthem68/BaselIIRat45/Arbeitspapier_B asellI.pdf,

Stand: 20.12 .2006

Taistra, Gregor (2004):

Rating und Unternehmensfinanzierung nach Basel II, Präsentation: PIMM Meeting, Danzig 2004, http://www.kfw.de/DE_Home/Research/Sonderthem68/BaselIIRat45/0401 Danzig.pdf, Stand: 20.12 .2006 


\section{Vavra, Terry Gwyn (1997):}

Improving Your Measurement of Customer Satisfaction - A Guide to Creating, Conducting, Analyzing and Reporting Customer Satisfaction Measurement Programs, Milwaukee 1997

\section{VR FACTOREM GmbH (2006):}

http://www.vr-factorem.de, Stand: 11.12.2006

Werani, Thomas (2004):

Bewertung von Kundenbindungsstrategieen in B-to-B-Märkten - Methodik und praktische Anwendung, Wiesbaden 2004

Wildner, Georg (2006):

Mehr als ein Slogan?, in: Effert, Detlef / Hanreich, Wilfried (Hrsg.) (2006), Ganzheitliche Beratung bei Banken, Wiesbaden 2006, S. 105-112 
Nino Raddao - 978-3-631-75326-2

Downloaded from PubFactory at 01/11/2019 06:11:43AM

via free access 


\section{Beiträge zum Controlling}

Herausgegeben von Wolfgang Berens

Band 1 Wolfgang Berens / Joachim Strauch: Due Diligence bei Unternehmensakquisitionen - eine empirische Untersuchung. Unter Mitarbeit von Thorsten Behrens und Julia Lescher. 2002.

Band 2 Andreas Siemes: Marktorientierte Kreditrisikobewertung. Eine empirische Untersuchung mittels Künstlicher Neuronaler Netze. 2002.

Band 3 Karl Christoph Heinen: Die Berücksichtigung von Kosten in der Konkurrenzanalyse. 2002.

Band 4 Thomas Mosiek: Interne Kundenorientierung des Controlling. 2002.

Band 5 Vera Südmeyer: Wettbewerbsvorteile durch strategisches Betriebsformenmanagement. Ein dynamischer Bezugsrahmen für Einzelhandelsunternehmen. 2003.

Band 6 Wolfgang Berens / Walter Schmitting (Hrsg.): Controlling im E-Business. Rückkehr zur Rationalität. 2004.

Band 7 René Bertelsmann: Entwicklung einer Controlling-Konzeption im verallgemeinerten Neuen Steuerungsmodell für Trăgerorganisationen der gesetzlichen Unfallversicherung. 2005.

Band 8 Mirko Tillmann: Risikokapitalbasierte Steuerung in der Schaden- und Unfallversicherung. Konzeption einer modellgestützten Risikoanalyse. 2005.

Band 9 Andreas Hoffjan: Risikorechnung bei industrieller Auftragsfertigung. Theoretische Konzeption und Anwendung für die Bauwirtschaft. 2006.

Band 10 Pascal Nevries: Die Marketingwirkungen von Börsengängen. Eine konzeptionelle Analyse. 2006.

Band 11 Klaus Segbers: Die Geschäftsbeziehung zwischen mittelstăndischen Unternehmen und ihrer Hausbank. Eine okonomische und verhaltenswissenschaftliche Analyse. 2007.

Band 12 Andreas Röhrig: Wirkungsorientiertes Controlling im politisch-administrativen System. Unter besonderer Berücksichtigung der Gestaltungsmőglichkeiten von öffentlichen Verwaltungen. 2008.

Band 13 Nino Raddao: Potenzialorientierte Kundensegmentierung zur Optimierung des Leistungsporttolios in der Firmenkundenbank. Konzeption und Implementierung einer efficient customization am Beispiel von Genossenschaftsbanken. 2009.

www.peterlang.de 


\title{
Die Geschäftsbeziehung zwischen mittelständischen Unternehmen und ihrer Hausbank
}

\author{
Eine ökonomische und verhaltenswissenschaftliche \\ Analyse
}

Frankfurt am Main, Berlin, Bern, Bruxelles, New York, Oxford, Wien, 2007. XXIV, 448 S., zahlr. Abb. und Tab.

Beiträge zum Controlling. Herausgegeben von Wolfgang Berens. Bd. 11 ISBN 978-3-631-55280-3 · br. $€ 77.70^{\star}$

Das Hausbankprinzip nimmt bei der Finanzierung mittelständischer Unternehmen in Deutschland traditionell eine zentrale Stellung ein. Diese Beziehung unterliegt aktuell einem starken Wandel. Als Konsequenz wird häufig eine Abkehr vom Hausbankprinzip gefordert. Diese Untersuchung widmet sich der Hausbankbeziehung aus einer interdisziplinären Sichtweise heraus. Es wird ein ganzheitliches Modell entwickelt, das sowohl ökonomische als auch verhaltenswissenschaftliche Aspekte umfasst. Danach erfordert eine Hausbankbeziehung von beiden Partnern kontinuierlich die Bereitschaft, den Geschäftspartner durch eine Übererfüllung von dessen Erwartungen emotional zu binden. Die Arbeit zeigt Mängel in der aktuellen Bankpraxis auf und leitet daraus praktisch-normative Gestaltungsempfehlungen ab.

Aus dem Inhalt: Ökonomische und verhaltenswissenschaftliche Ansätze einer Hausbankbeziehung · Einflussfaktoren auf das Hausbankprinzip · Darstellung des Forschungsstandes zu Einflussfaktoren auf Kreditzins und -verfügbarkeit sowie Besicherung - Psychologische Fundierung des Vertrauenskonstruktes als Differenzierungsmerkmal · Entwicklung eines Wirkungsmodells der Kundenbindung als Grundlage für ein interdisziplinäres Verständnis einer Hausbankbeziehung · Ableitung praktisch-normativer Gestaltungsempfehlungen

Frankfurt am Main - Berlin - Bern - Bruxelles - New York · Oxford · Wien

Auslieferung: Verlag Peter Lang AG

Moosstr. 1, $\mathrm{CH}-2542$ Pieterlen

Telefax 0041 (0) $32 / 3761727$

*inklusive der in Deutschland gültigen Mehrwertsteuer

Preisänderungen vorbehalten

Homepage http://www.peterlang.de 